Bioinspired oxidation reactions of phenols with dinuclear copper complexes

Angelina Prohofieva Göttingen 19th September 2007 


\title{
Bioinspired oxidation reactions of phenols with dinuclear copper complexes
}

\author{
Dissertation \\ zur Erlangung des Doktorgrades \\ der Mathematisch-Naturwissenschaftlichen Fakultäten \\ der Georg-August-Universität zu Göttingen \\ vorgelegt von
}

Diplom-Chemikerin

Angelina Prokofieva

aus Kiev, Ukraine

Göttingen, den 19.09.07 

D7

Referent: Prof. Dr. Franc Meyer

Korreferent: Prof. Dr. George M. Sheldrick

Tag der mündlichen Prüfung: 01.11.2007 
Ich erkläre hiermit an Eides statt, daß ich diese Arbeit selbständig und ohne unerlaubte Hilfsmittel angefertigt habe. 
Introduction

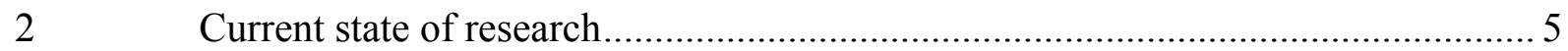

2.1 Metalloenzymes with a copper containing active site....................................... 5

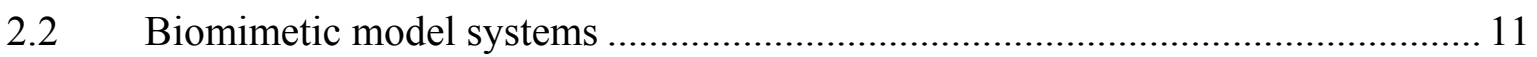

3 Homogeneous copper catalyzed reactions ...................................................... 15

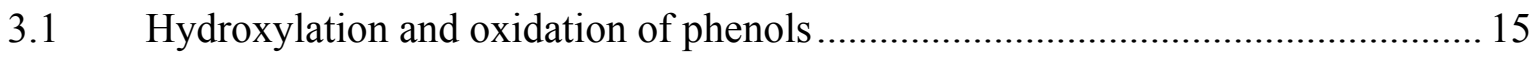

3.2 Oxidative polymerization and coupling of phenols ............................................ 19

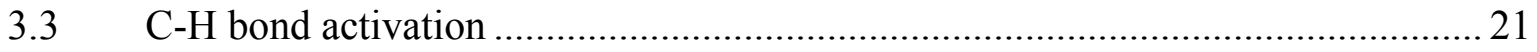

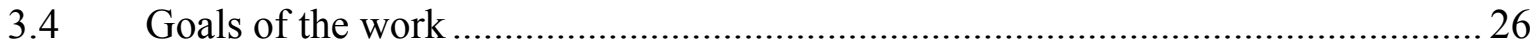





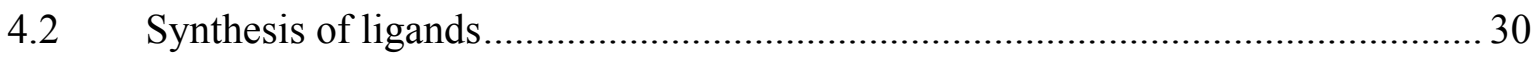

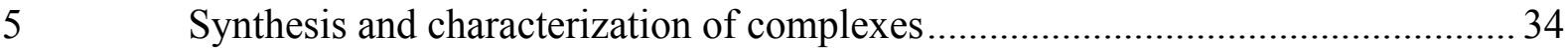

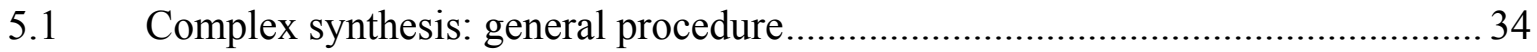

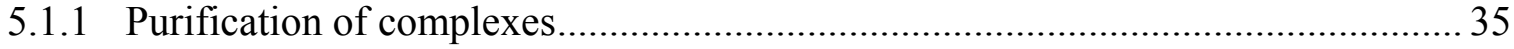

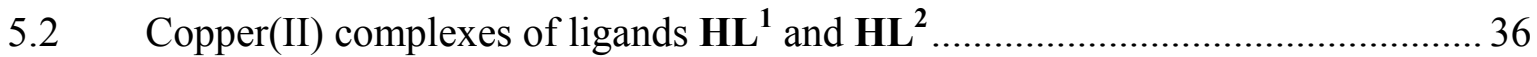

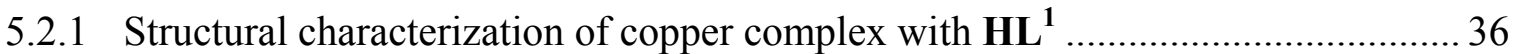

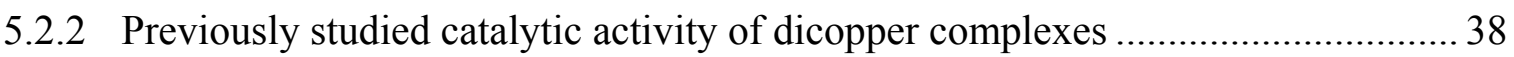

5.2.3 Equilibria in solution and structural characterization of complexes with $\mathbf{H L}^{2}$ : unusual copper-dioxygen mediated amine to $N$-oxide transformation of $\mathbf{H L}^{2} \ldots . . .40$

5.3 Characterization of a dicopper complex with the new ligand $\mathbf{H L}^{3}$.......................4 47

5.4 Copper complexes of the new bioinspired ligand $\mathbf{H L}^{4}$..................................... 49

5.4.1 Elucidation of species distribution at different $\mathrm{pH}$ values .................................. 49

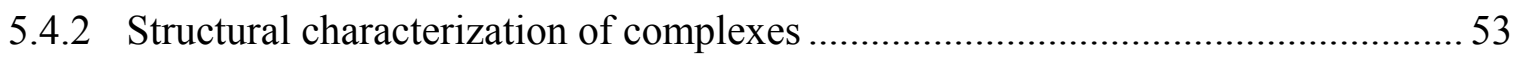

5.4.3 Spectroscopic properties of the complexes and equilibria in solution ....................59

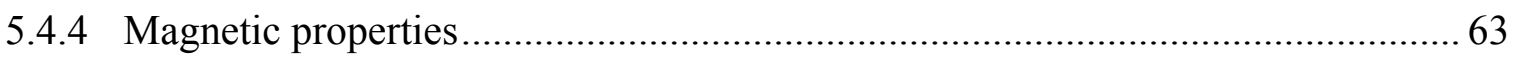

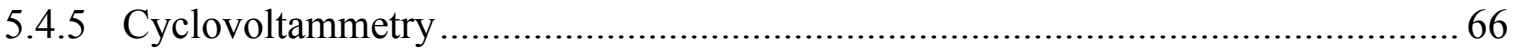

5.5 Characterization of a copper complex with the new ligand $\mathbf{H L}^{\mathbf{5}}$...........................6

5.6 Comparison of the spectroscopic and structural features of the copper complexes...

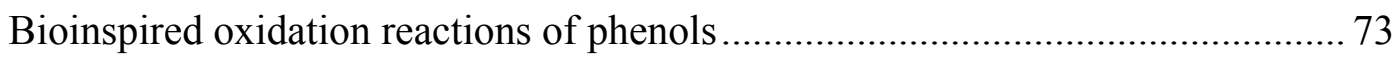

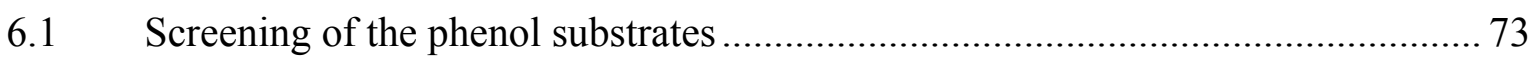

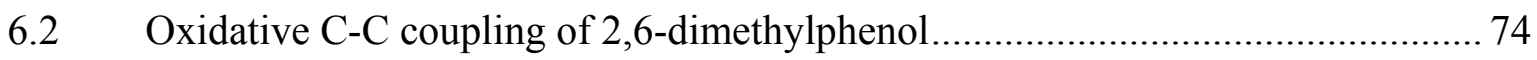

6.3 Oxidative C-C coupling of 2,4,6-trimethylphenol …......................................... 76 
7.1 Determination of the optimal reaction conditions for C-C-coupling of TMP........ 79

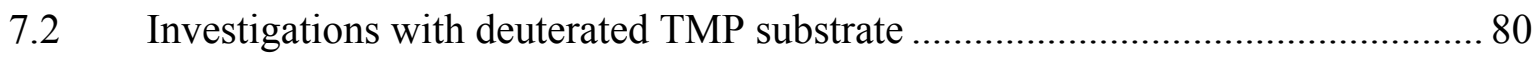

7.3 Discussion of the nature of the UV/vis Charge Transfer band of dicopper

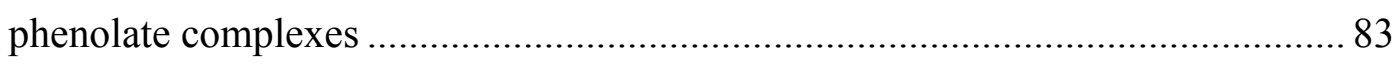

7.4 Determination of the nature of the adduct formed upon the addition of TMP....... 85

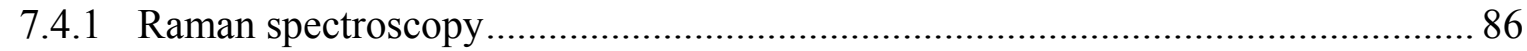



7.5 Determination of the amount of coordinated phenol ........................................... 92

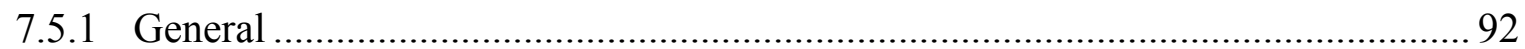



7.5.3 Job plot of 4-methylphenol and 4-tert-butylphenol with dicopper complex $\mathbf{5 a}$.... 97

7.5.4 Job plot of 4-hydroxybenzamide and pentafluorophenol with dicopper complex 5a

7.6 Mechanistic studies of C-C coupling of TMP under anaerobic conditions 101

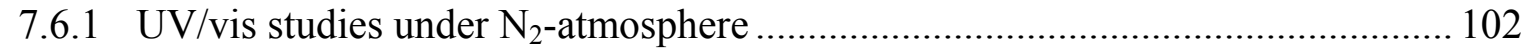

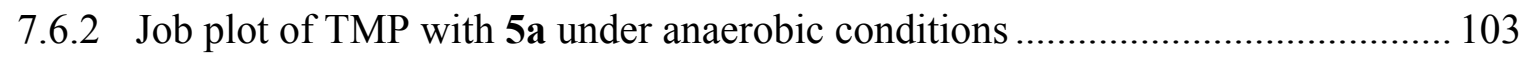

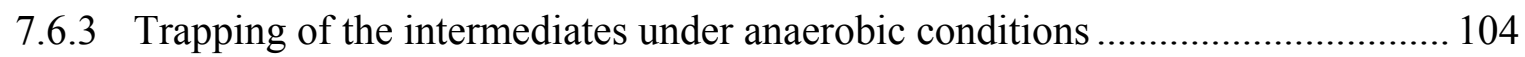

7.6.4 Evidence for the formation of a mixed-valence $\mathrm{Cu}^{\mathrm{I}} \mathrm{Cu}^{\mathrm{II}}$ species ......................... 106

7.7 Coordination behaviour of the new ligand $\mathbf{H L}^{\mathbf{5}}$ towards copper(I) ..................... 111

7.8 Time-dependent concentration profiles of TMP, TMBB and TMSQ during

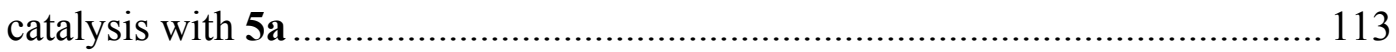

7.9 Determination of the $\mathrm{O}_{2}$ derived by-product in the C-C coupling reaction .......... 114 Adducts with model substrate molecules ............................................................. 116

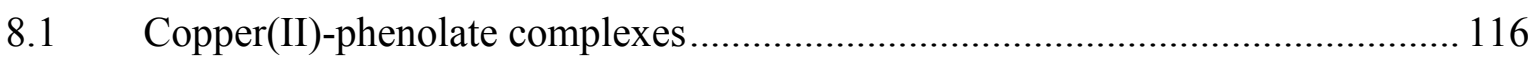

8.2 Characterization of the adducts with 4-hydroxybenzamide and pentafluorophenol..

8.3 4,4'-isopropylidene-bis(2,6-dimethylphenol) and 4,4'-(hexafluoroisopropylidene) diphenol as model substrates for TMBB …...................................................... 126

8.4 A tetrachlorocatechol-adduct of dicopper(II) complex 5a ................................. 132

9 Oxidative ortho-C-C coupling of 4-ethylphenol catalyzed by 8 ......................... 139

$10 \quad$ Nucleophilic 1,6-addition of $\mathrm{MeOH}$ to 2,4,6-trimethylphenol .............................. 146

11 Oxidative C-O coupling of 4-bromo-2,6-dimethylphenol catalyzed by $\mathbf{5 a}$......... 149

12 Discussion of the proposed mechanism for C-C coupling of TMP ................... 153 


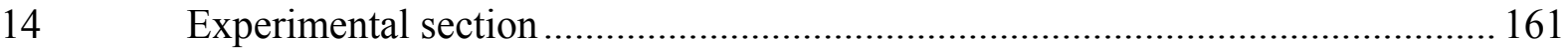

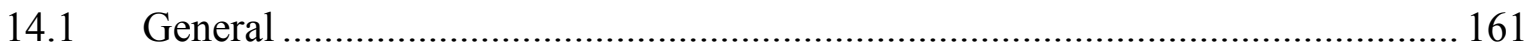

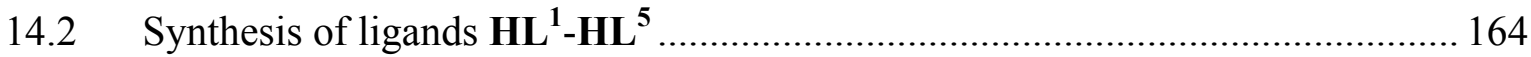

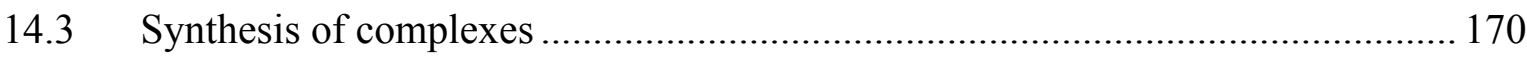

14.4 Synthesis of 3,3',5,5' -tetramethylstilbene-4,4'-quinone (TMSQ) ....................... 189

14.5 Synthesis of 4,4'-dihydroxy-3,3',5,5'-tetramethylbibenzyl (TMBB) ................... 190

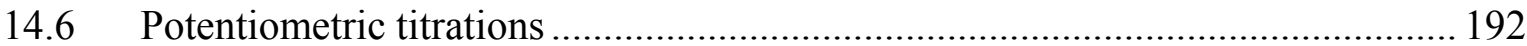

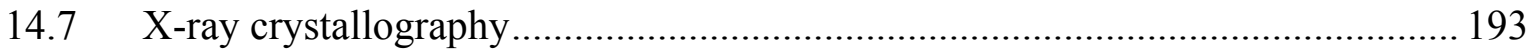



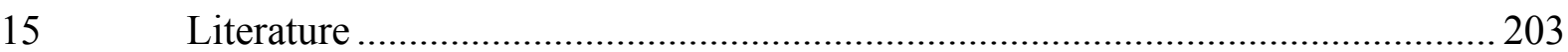



The world is all that is encased here: life, death, people, and everything else that surrounds us. The world is incomprehensible. We won't ever understand it. We won't ever unravel its secrets. Thus we must treat the world as it is: a sheer mystery.

The Wheel of Time

Carlos Castaneda

...to my dear parents...

19 September 2007 



\begin{tabular}{|c|c|c|c|}
\hline $\mathrm{Ar}$ & aromatic ring & HMQC & $\begin{array}{l}\text { Heteronuclear Multiple Quantum } \\
\text { Corrrelation }\end{array}$ \\
\hline 4-AP & 4-hydroxybenzamide & IR & infra red \\
\hline $\mathrm{Bz}$ & 1-methyl benzimidazole & $J$ & coupling constant \\
\hline $\mathrm{CT}$ & Charge Transfer & $\mathrm{KO} t \mathrm{Bu}$ & potassium-tert-butylate \\
\hline $\mathrm{CV}$ & Cyclovoltammetry & $K_{\mathrm{d}}$ & dissociation constant \\
\hline $\mathrm{CH}_{2} \mathrm{Cl}_{2}$ & Dichloromethane & $\mathrm{L}$ & Ligand \\
\hline calcd. & Calculated & LMCT & Ligand-Metal-Charge-Transfer \\
\hline $\mathrm{CN}$ & coordination number & L-Dopa & 4-(2-aminoethyl)benzene-1,2-diol \\
\hline$C D_{3}$-TMP & deuterated 2,4,6-trimethylphenol & $\lambda$ & wavelength \\
\hline$d$ & Distance & $\lambda_{\mathrm{ex}}$ & wavelength of laser excitation \\
\hline $\mathrm{DMF}$ & $N, N$-dimethylformamide & $m$ & Meta \\
\hline DMP & 2,6-dimethylphenol & M & Molar \\
\hline DMBrP & 4-bromo-2,6-dimethylphenol & $\max$ & Maximum \\
\hline MDP & 4-methoxy-2,6-dimethylphenol & $\mathrm{Me}$ & Methyl \\
\hline DTBC & 3,5-di-tert-butylcatechol & $\mathrm{MeCN}$ & Acetonitrile \\
\hline DPQ & $\begin{array}{l}\text { 3,3',5,5' -tetramethyl-4,4'- } \\
\text { diphenoquinone }\end{array}$ & $\mathrm{MeOH}$ & Methanol \\
\hline $\mathrm{EtOH}$ & Ethanol & MS & Mass Spectrometry \\
\hline $\mathrm{Et}_{2} \mathrm{O}$ & Diethylether & 4-MeP & 4-methylphenol \\
\hline$\varepsilon$ & extinction coefficient & $\mathrm{M} t \mathrm{BuP}$ & 2-methyl-4-tert-butylphenol \\
\hline$E$ & electrochemical potential & $3-N B A$ & 4-nitrobenzylalcohol \\
\hline EI & Electron Impact & NMR & Nuclear Magnetic Resonance \\
\hline ESI & Electron Spray Ionisation & $o$ & Ortho \\
\hline EPR & Electron Paramagnetic Resonance & PPE & poly(2,6-dimethylphenylene ether) \\
\hline Et & Ethyl & $p$ & Para \\
\hline 4-EtP & 4-ethylphenol & py & Pyridyl \\
\hline FAB & Fast Atom Bombardment & PFP & Pentafluorophenol \\
\hline $\mathrm{HOCF}_{3} \mathrm{BP}$ & $\begin{array}{l}\text { 4,4'-(hexafluoroisopropylidene) } \\
\text { diphenol }\end{array}$ & Red & Reduction \\
\hline $\mathrm{HOCH}_{3} \mathrm{BP}$ & $\begin{array}{l}\text { 4,4'-isopropylidene-bis(2,6- } \\
\text { dimethylphenol) }\end{array}$ & $\rho$ & paramagnetic impurities \\
\hline HMBC & $\begin{array}{l}\text { Heteronuclear Multiple Bond } \\
\text { Correlation }\end{array}$ & $\mathrm{S} / \mathrm{C}$ & substrate/catalyst ratio \\
\hline
\end{tabular}




\begin{tabular}{|c|c|}
\hline sh & shoulder \\
\hline TCC & tetrachlorocatechol \\
\hline TMP & 2,4,6-trimethylphenol \\
\hline 2,5,6-TMP & 2,5,6-trimethylphenol \\
\hline 2,3,5-TMP & 2,3,5-trimethylphenol \\
\hline$t \mathrm{BuP}$ & 4-tert-butylphenol \\
\hline$T I P$ & $\begin{array}{l}\text { temperature-independent } \\
\text { paramagnetism }\end{array}$ \\
\hline TMBD & $\begin{array}{l}\text { 3,3',5,5'-tetramethylbiphenyl-4,4'- } \\
\text { diol }\end{array}$ \\
\hline TMSQ & $\begin{array}{l}3,3^{\prime}, 5,5 \text { '-tetramethylstilbene- } 4,4^{\prime} \text { - } \\
\text { quinone }\end{array}$ \\
\hline $\mathrm{T}$ & temperature \\
\hline TON & turnover numbers \\
\hline TACN & triazocyclononane \\
\hline TMBB & $\begin{array}{l}\text { 4,4'-dihydroxy-3,3',5,5'- } \\
\text { tetramethylbibenzyl }\end{array}$ \\
\hline$\chi_{M}$ & magnetic susceptibility \\
\hline$\chi_{M} T$ & product of magnetic susceptibility \\
\hline$v$ & wavenumber \\
\hline
\end{tabular}




\section{Introduction}

Copper has been known as one of the important raw materials for a long time, although the content of copper in the earth's crust is only $0.003 \%$. Copper has played a significant part in the history of mankind, which has used the easily accessible uncompounded metal for nearly 10.000 years. Civilizations in places like Egypt and Greece showed early evidence of using copper. During the Roman Empire copper was principally mined on Cyprus, hence the origin of the name of the metal as Cyprium, "metal of Cyprus", later shortened to Cuprum. It is a ductile metal with an excellent electrical conductivity, and it finds extensive use as an electrical as well as thermal conductor, as building material and as a component of various alloys (e.g. brass $(\mathrm{Cu} / \mathrm{Zn})$, bronzes $(\mathrm{Cu} / \mathrm{Al} / \mathrm{Si})$, precious metal alloys).

In modern chemistry copper compounds play a vital role in organic synthesis as (co-)catalysts for a whole plethora of reactions. For example, copper(I) salts catalyze the decomposition of the diazonium salts, an important step in the Sandmeyer reaction. Organometallic copper complexes, such as cupric and cuprous chlorides, are known to be active in the oxychlorination of ethylene to yield 1,2-dichloroethane. ${ }^{1,2}$ Other well-known catalytic applications of organometallic copper complexes include the ring-opening of epoxides and the addition to alkynes. ${ }^{3,4}$ The most prominent example of copper acting as co-catalyst is in Pdcatalyzed Wacker-type oxidations of alkenes, where copper salts mediate the reduction of palladium(II) by molecular oxygen. ${ }^{5,6,7}$

The fundamental coordination chemistry of copper with respect to the role that coordination effects play in both synthetic and biological systems has been reviewed. ${ }^{8,9,10}$ A large set of copper complexes are known to exist, wherein the oxidation state of the copper ion(s) can range from 0 to $+4 .^{8}$ The most important and common for both biological and synthetic systems are the +1 and especially the +2 oxidation states. Both the coordination number $(\mathrm{CN})$ and the geometry of a copper ion are highly dependent on the metal-oxidation state. Thus, for a $d^{10} \mathrm{Cu}^{\mathrm{I}}$ ion, coordination numbers from 2 to 5 are observed, with 4 being most common $(\mathrm{CN}$ 2: $\left.\left[\mathrm{CuCl}_{2}\right]^{-}, \mathrm{CN} 3:\left[\mathrm{Cu}(\mathrm{CN})_{3}\right]^{-}\right)$. The most common coordination environments are linear $(\mathrm{CN}$ $=2)$, trigonal planar $(\mathrm{CN}=3)$ and tetrahedral $(\mathrm{CN}=4)$ geometries. In contrast, a copper(II) $d^{9}$ ion usually prefers a coordination number of 5 or 6 , resulting in a trigonal bipyramidal geometry (Figure 1 (a)) or a tetragonal pyramidal coordination environment (Figure 1 (b)) for 
the former and an octehedral geometry for the latter; usually a strong Jahn-Teller effect (four ligands are strongly bound in the equatorial plane, with one (or two) additional ligand(s) less strongly coordinated in the axial position(s)) is observed.



a

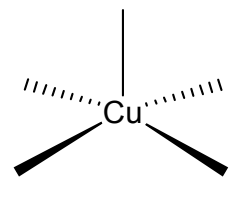

b

Figure 1. Trigonal bipyramidal (a) or tetragonal-pyramidal (b) coordination environment of the copper ion $(\mathrm{CN}=5)$.

These differences in preferred geometry (and the related coordination numbers) for $\mathrm{Cu}^{\mathrm{I}} \mathrm{vs}$. $\mathrm{Cu}^{\mathrm{II}}$ lead to drastic coordination changes in the case of a redox reaction between these two above-mentioned oxidation states. The standard aqueous reduction potentials show that copper(I) is unstable in aqueous solution due to a disproportionation reaction to form metallic copper and copper(II) $\left(2 \mathrm{Cu}^{+} \rightarrow \mathrm{Cu}^{0}+\mathrm{Cu}^{2+}, E^{0}=+0.37 \mathrm{~V}\right)$.

Copper is essential for various biological processes occurring in all higher plant and animal species and it is the third most abundant trace element found in the human body, after iron and zinc. When copper is taken up by the body, it is first absorbed in the gut, and from there transported to the liver as an albumin-bound complex. Copper is found in a wide variety of metalloenzymes, including the active sites of cytochrome $c$ oxidase and the enzyme superoxide dismutase (containing copper and zinc). The main biological role of copper is centered on redox-chemistry (electron transfer or oxidation/oxygenation of organic substrates). The functions of the active sites of copper metalloproteins can be divided in four different types: (i) metal ion uptake, storage and transport, (ii) dioxygen activation, storage and transport, (iii) electron transfer, and (iv) catalytic conversion of organic molecules. Originally, copper centers in biological systems were classified in three groups on the basis of their spectroscopic features; however the immense advances in protein crystallography have led to an expansion of the classification system for the currently known biological coppercontaining systems. There are now seven different classes of copper systems, within which copper is involved in completely different chemical processes, from catalytic oxidation of organic substrates (type 3 ) to the reduction of organic substrate molecules $\left(\mathrm{Cu}_{\mathrm{B}}\right.$ type). ${ }^{11}$ 
Recent discoveries of various novel copper containing proteins pushed the area of bioinorganic chemistry even further into the design of small-molecule models (structural and functional) for the active sites of copper metalloenzymes. Such bioinspired and biomimetic copper complexes serve two different goals. Design and synthetic use of accurate model complexes will lead to a deeper understanding of the functioning or characteristics (e.g. spectroscopic features) of the enzyme active site and in the meantime will provide a great opportunity to apply these principles and copper complexes for the selective transformation of substrate molecules in homogeneous catalysis.

The work described in this thesis was born out of inspiration fuelled by the versatile possibilities in the design and practical use of model copper complexes.

Preorganized dinuclear transition metal complexes have received a lot of attention over the past several years, mainly due to the increasing interest in cooperative effects between individual metal centers. ${ }^{12}$ The body of work detailed in the following chapters is based on pyrazole-based ligands with chelating side arms in the 3- and 5-positions of the heterocyclic core, which have been developed as valuable scaffolds for further studies: the anionic pyrazolate has a high tendency to span two metal ions in a bridging fashion, while the individual coordination spheres as well as the intramolecular metal-metal separation can be tuned by appropriate alterations of the appended chelate substituents. ${ }^{13,14,15,16,17}$

New bioinspired pyrazole ligands have been designed, synthesized and fully characterized. The coordination chemistry of oligonuclear copper complexes with these different pyrazolebased ligands was thoroughly investigated, which has led to detailed insight into the apparently high flexibility of the ligand system whilst coordinating copper and has enabled a systematic determination of the spectroscopic properties of a range of different $\mathrm{Cu}$ complexes. By varying the number of additional donor-functionalities, the geometry of the copper ions in the complexes could be manipulated, whereas modification of the spacer length between the pyrazolate core and the chelating side-arm substituents provided great opportunities for control over the intramolecular metal-metal distance.

Alongside these investigations into the coordination chemistry, studies were performed on the catalytic activity of these novel copper complexes. Particular attention was given to the oxidative C-C coupling of 2,6-dimethylphenol (DMP) and 2,4,6-trimethylphenol (TMP), as 
well as on the 1,6-nucleophilic addition of several small molecules to TMP. Furthermore, dicopper complexes with suitable derivatives of the general class of phenolic substrates that showed no tendency for any $\mathrm{Cu}$-mediated transformation were prepared and characterized in order to determine possible (structural) intermediates of the catalytic reactions studied.

All in all, the results obtained in this work have provided a better understanding of the reaction mechanism behind, in particular for the $\mathrm{C}-\mathrm{C}$ oxidative coupling of TMP. 


\section{Current state of research}

\subsection{Metalloenzymes with a copper containing active site}

One of the important functions performed by metalloproteins is respiration. There are three known classes of dioxygen transport proteins: the hemoglobin-myoglobin family (containing a mononuclear heme center), hemocyanins (which feature a dinuclear copper active site), and hemerythrins (with a dinuclear non-heme iron active site). ${ }^{18,19,20}$ In these proteins, a diatomic $\mathrm{O}_{2}$ molecule binds to an iron or copper ion present in the active site of the metalloenzyme, without the occurrence of any irreversible electron transfer or redox reactions. In hemoglobin and myoglobin the dioxygen binding site is an iron-porphyrin complex. In the oxy form the $\mathrm{Fe}$ ion adopts a square bipyramidal geometry - four $\mathrm{N}$-atoms of the porphyrin ring are coordinated in the equatorial plane, while a histidine residue and end-on bound dioxygen molecule occupy the apical positions. The other two classes of respiratory metalloproteins employ dinuclear active sites for the dioxygen-binding reactions. In hemerythrin the $\mathrm{O}_{2}$ molecule is bound at a terminal site in the $\mathrm{Fe}_{2}$ unit, concomitant with oxidation of the bimetallic center (generation of hydroperoxide).

In hemocyanin (a type 3 copper active site found in molluscs and arthropods), reversible binding of dioxygen is performed by a pair of copper ions (Figure 2).
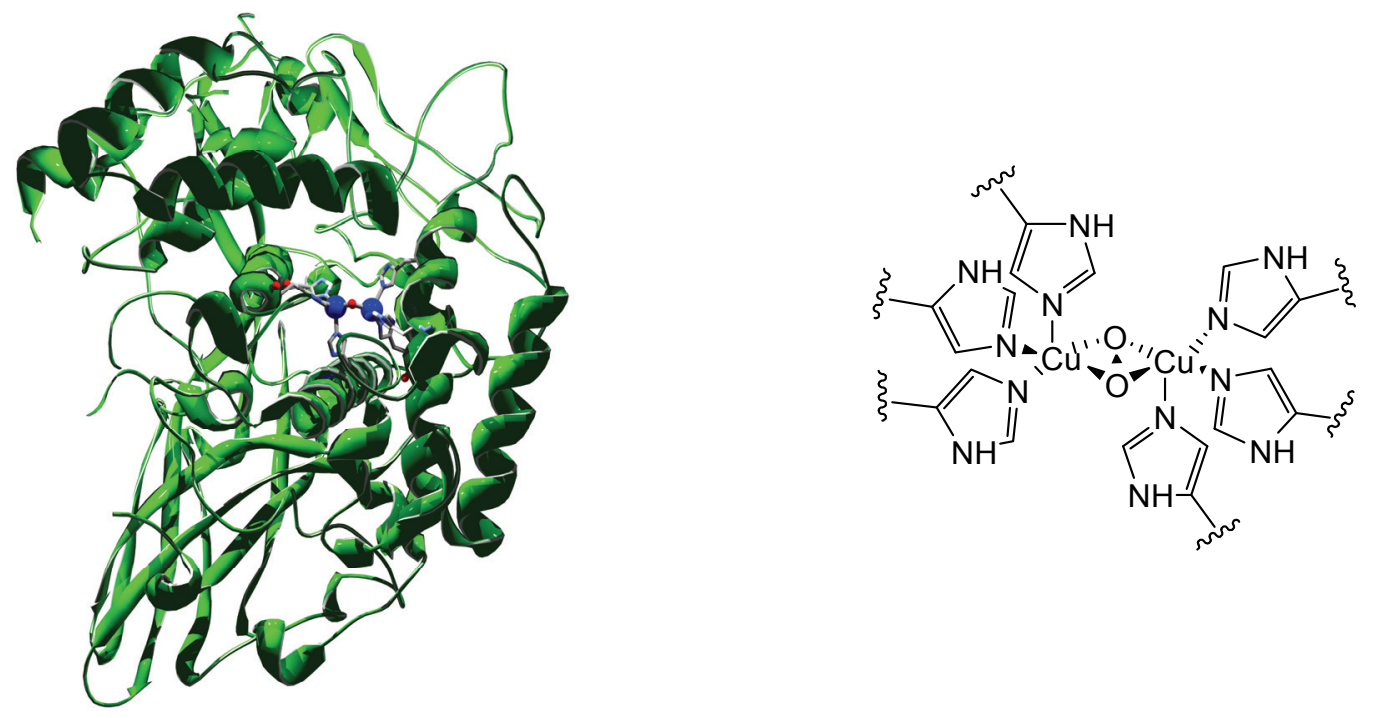

Figure 2. Molecular structure of hemocyanin (left) and the corresponding active site in the oxy form (right). 
The assumption that binding of dioxygen is reversible and concomitant with reversible transformation of dicopper(I) (deoxy form) into the dicopper(II) (oxy form) species is in good agreement with the $\mathrm{Cu}^{\mathrm{II}} / \mathrm{Cu}^{\mathrm{I}}$ reduction potential $\left(E^{0}\left(\mathrm{Cu}^{\mathrm{II}} / \mathrm{Cu}^{\mathrm{I}}\right)=+0.15 \mathrm{~V}\right)$. Both the oxy and the deoxy form of the hemocyanin active site have been structurally characterized. ${ }^{21,22,23,24}$ Dioxygen is coordinated as a side-on $\left(\mu-\eta^{2}: \eta^{2}\right)$ peroxo ligand, with the two copper ions being $3.60 \AA$ apart while each $\mathrm{Cu}$ is ligated by three histidine residues.

The currently known biological copper systems that are involved in oxidation catalysis can be categorized in three classes: type 2, type 3 and type 4.

Type 2 copper active sites are found in different oxidases (e.g. galactose oxidase (Figure.3): oxidation of primary alcohols) ${ }^{25}$ and oxygenases (dopamine- $\beta$-hydroxylase: $\mathrm{C}-\mathrm{H}$ bond activation of benzylic substrates, ${ }^{26}$ and phenylalanine hydroxylase: hydroxylation of aromatic substrates ${ }^{27}$ ) as well as in $\mathrm{CuZn}$ superoxide dismutase (disproportionation of $\mathrm{O}_{2}^{-}$superoxide anion). ${ }^{28}$

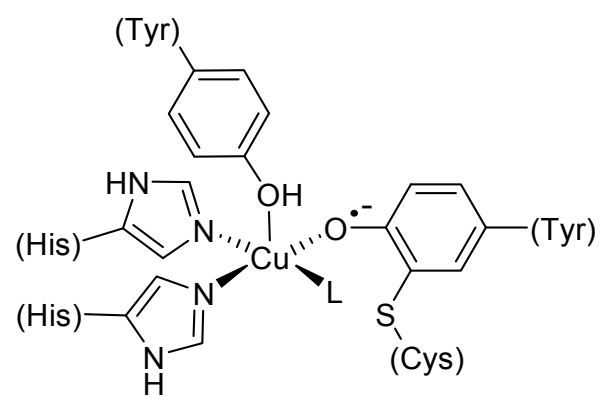

Figure 3. Structure of the active site of galactose oxidase (active form), $\mathrm{L}=\mathrm{H}_{2} \mathrm{O}$.

A type 2 or normal $\mathrm{Cu}^{2+}$ site shows no detectable absorption features in the $\mathrm{UV} / \mathrm{vis}$ region and an EPR line shape reminiscent of common low-molecular weight mononuclear copper complexes $\left(\mathrm{A}_{I P}>140 \cdot 10^{-4} \mathrm{~cm}^{-1}\right)$. In the oxidized state, their colour is light blue because of weak $d$ - $d$ transitions within the single $\mathrm{Cu}^{\mathrm{II}}$ ion. The coordination sphere around $\mathrm{Cu}$, which has either square planar or distorted tetrahedral geometry, contains four ligands with $\mathrm{N}$ and/or $\mathrm{O}$ donor atoms. ${ }^{29}$ Galactose oxidase represents an example of a free radical metalloenzyme active site and it catalyzes the oxidation of galactose and other primary alcohols under twoelectron reduction of $\mathrm{O}_{2}$ to $\mathrm{H}_{2} \mathrm{O}_{2}$, whereas dopamine- $\beta$-hydroxylase catalyses the hydroxylation of 4-(2-aminoethyl)benzene-1,2-diol (L-Dopa) into the neurotransmitter 
noradrenaline (Scheme 1) by selective benzylic C-H bond activation, thereby installing an $\mathrm{OH}$-group at the $\alpha$-carbon. The latter system is therefore classified as an oxygenase enzyme.<smiles>NCCc1ccc(O)c(O)c1</smiles>

dopamine dopamine- $\beta$-hydroxylase

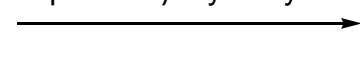

L-noradrenaline

Scheme 1. Hydroxylation of dopamine into noradrenaline.

Type 3 copper sites in their oxy form are characterized by a strong absorption band in the near UV region $\left(\lambda_{\max }=330 \mathrm{~nm}\right)$. These type 3 copper centers are believed to consist of two copper ions that are antiferromagnetically coupled. The type 3 class of proteins comprises hemocyanin, ${ }^{30}$ tyrosinase $^{31}$ and catechol oxidase. ${ }^{32}$ Their active sites are very similar, containing a dicopper core in which both $\mathrm{Cu}$ ions are ligated by three $\mathrm{N}$-bound histidine residues. All three metalloproteins are capable of binding dioxygen reversibly at ambient conditions. Structural insight from X-ray crystallography is now available for all three metalloproteins. ${ }^{33,34,35}$ Antiferromagnetic coupling of the two $\mathrm{Cu}^{\text {II }}$ ions in the oxy state of these metalloproteins leads to EPR-silent behaviour.

Catechol oxidase (Figure 4) performs the oxidation of catechols to $o$-quinones with $\mathrm{O}_{2}$ as the oxidant. This two-electron substrate oxidation is coupled to the reduction of $\mathrm{O}_{2}$ to water, i.e. no oxygen atom(s) stemming from $\mathrm{O}_{2}$ are incorporated in the substrate. During the catalytic cycle, each copper ion switches between the +1 and +2 oxidation states and thus provides only a single electron, but the cooperative effect of two adjacent copper ions enables twoelectron redox reactions to occur at such type 3 active sites. 


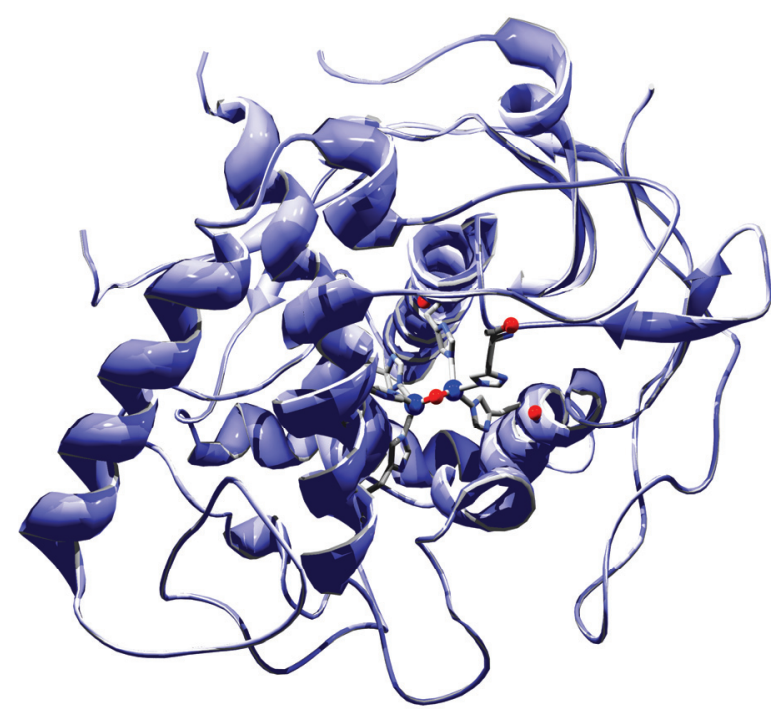

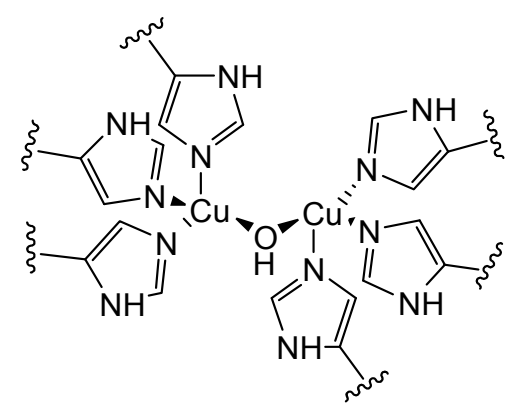

Figure 4. Molecular structure of catechol oxidase (left) and the corresponding active site in the met form (right).

In the deoxy form both copper ions are in the +1 oxidation state and the metal-metal distance is around $4.40 \AA$, while in the oxidized met form the $\mathrm{Cu}$ ions are ligated by three N-bound histidine residues with a copper-copper distance equal to $2.90 \AA$ and an additional bridging $\mathrm{OH}$-ligand. The third representative of type 3 copper proteins is tyrosinase (Figure 5), which functions as a catalyst in the oxidation of phenolic substrates to catechols (cresolase activity) as well as in the (subsequent) oxidation of catechols to $o$-quinones (catecholase activity).

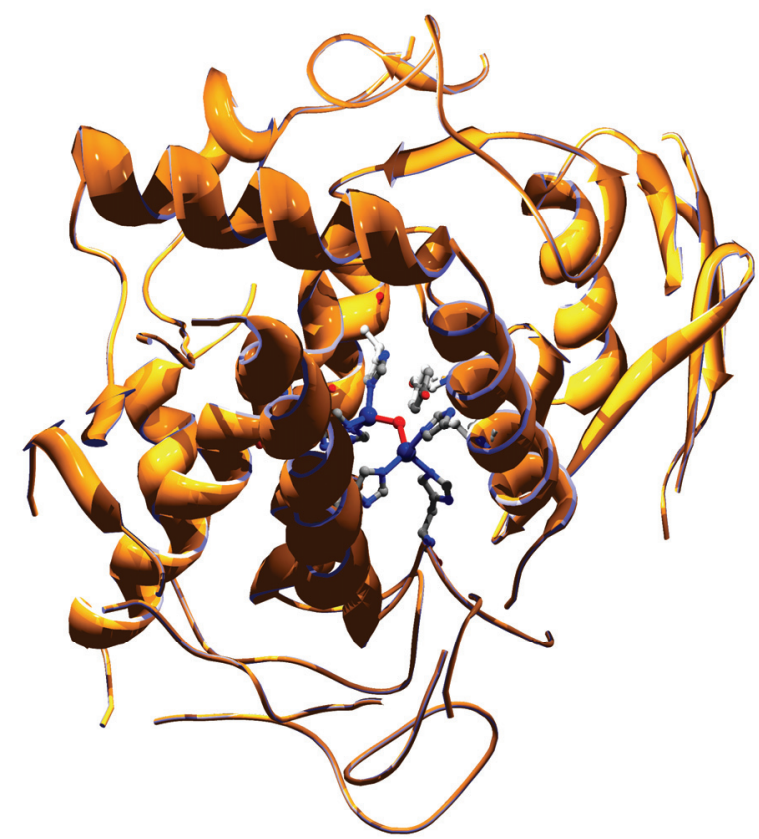

Figure 5. Molecular structure of tyrosinase. 
Oxidation of phenolic substrates to catechols includes transfer of only one O-atom, originating from $\mathrm{O}_{2}$, to a substrate molecule in an oxygenation step (Scheme 2).

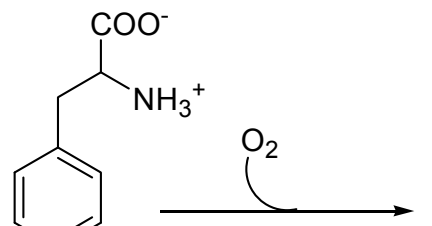

(a) tyrosine<smiles>[NH3+]C(Cc1ccc(O)c(O)c1)C(=O)[O-]</smiles>

L-DOPA (b)

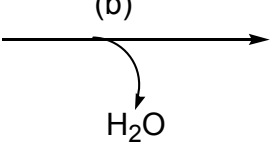<smiles>[NH3+]C(CC1=CC(=O)C(=O)C=C1)C(=O)[O-]</smiles>

Scheme 2. Oxidation of tyrosine by tyrosinase: cresolase (a) and catecholase (b) activity.

The recent elucidation at high resolution of the solid-state structure of tyrosinase by X-ray crystallographic methods has provided much information and allowed for a detailed mechanistic picture of the catalytic cycle to be proposed. ${ }^{31,35}$ It starts with a $\mathrm{Cu}^{\mathrm{I}} \mathrm{Cu}^{\mathrm{I}}$ species (the deoxy form) - the two $\mathrm{Cu}$ centers are denoted $\mathrm{Cu}^{\mathrm{A}}$ and $\mathrm{Cu}^{\mathrm{B}}$ - which reversibly binds $\mathrm{O}_{2}$ to form the common $\mathrm{Cu}^{\mathrm{II}}\left(\mu-\eta^{2}: \eta^{2}\right.$-peroxo) $\mathrm{Cu}^{\mathrm{II}}$ species (oxy form) that is pivotal to all type 3 dicopper sites. The substrate then docks to one of the metal ions of the oxy state (most likely $\mathrm{Cu}^{\mathrm{A}}$ ) and is properly oriented through interactions with histidine residues to enable hydroxylation of the aromatic ring. To this end, the O-O axis of the peroxo ligand has been suggested to rotate in order to point towards the phenolic ring, leading to electrophilic attack of the $\mathrm{Cu}\left(\mu-\mathrm{O}_{2}\right) \mathrm{Cu}$ moiety on the ring with concomitant cleavage of the O-O bond. ${ }^{35}$ The diphenolic intermediate ends up bound in a bidentate fashion and is subsequently released as the $o$-chinone to regenerate the deoxy form of the dicopper center. It should be noted though that alternative mechanistic proposals are being discussed, ${ }^{36}$ and even the site of substrate binding $\left(\mathrm{Cu}^{\mathrm{A}}\right.$ or $\left.\mathrm{Cu}^{\mathrm{B}}\right)$ is not yet fully clarified. Similar considerations apply to the mechanism of action in catechol oxidase, where important open questions comprise the exact mode of substrate and product binding (either to one or two $\mathrm{Cu}$ ions), and where several scenarios have been proposed for the catalytic cycle..$^{33,35}$ 

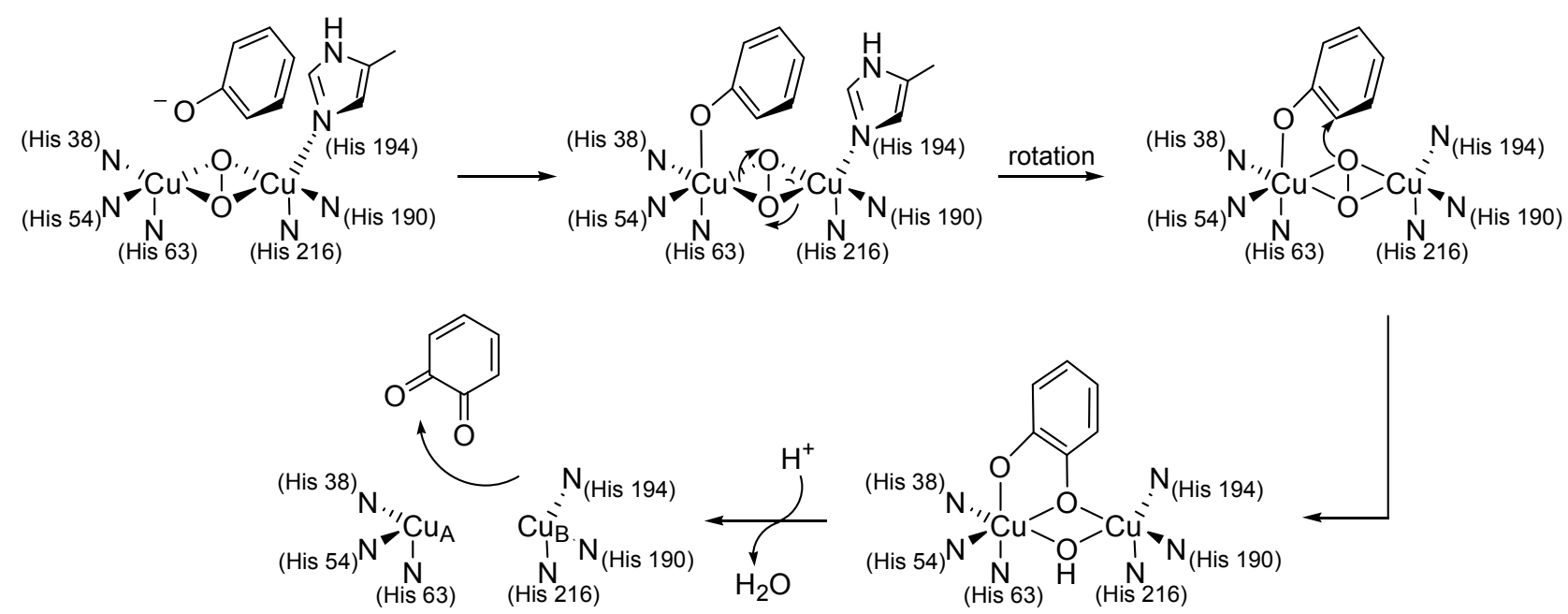

Scheme 3. Proposed catalytic cycle for the oxidation of phenol to catechol by the tyrosinase active site. ${ }^{35}$

Type 4 copper sites are metalloproteins that contain both type 2 and type 3 copper centers. Together, they form a triangular-shaped trinuclear active site, such as found in laccase (polyphenol oxidase) ${ }^{37,38}$ and ascorbate oxidase (Figure 6). ${ }^{39}$

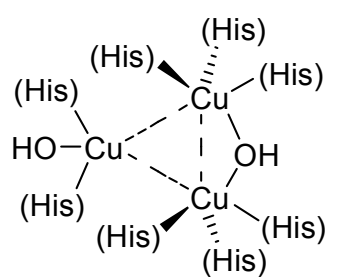

Figure 6. Structure of the active site of ascorbate oxidase.

The blue protein ascorbate oxidase belongs to the group of "blue" oxidases together with laccase and ceruloplasmin. These are multi-copper enzymes that catalyze the one-electron oxidation of specific substrates (no oxygen atom(s) from $\mathrm{O}_{2}$ are incorporated into the substrate) concomitant with the (overall) four-electron reduction of molecular oxygen to water. 


\subsection{Biomimetic model systems}

Many of today's studies are focussed on the design of small model complexes that can aid in understanding the principle mechanisms behind the catalytic activity displayed by metalloenzyme active sites. These model biomimetic and/or bioinspired copper complexes assist in the development of new catalysts for selective oxidations of organic substrates, preferably under mild and environmetally being conditions. Considerable attention has been given to the development of useful catalytic systems for oxidations with dioxygen, which is also very attractive from an industrial and economical point of view. The feasibility of such $\mathrm{O}_{2}$-based oxidation catalysis has recently been demonstrated with different dinuclear model compounds for the galactose oxidase active site. In the presence of these complexes and with $\mathrm{O}_{2}$, a range of alcohols were catalytically oxidized to the corresponding aldehydes. ${ }^{40,41}$

One of the first examples of a functional model complex for the tyrosinase active site was prepared by Karlin et al., wherein intramolecular hydroxylation of the phenyl group, acting as a bridgehead between two tridentate binding pockets, was observed (Scheme 4). In general, complexes derived from these dinucleating ligands feature symmetrically arranged, threecoordinate copper centers with nitrogen donors, because this arrangement simulates the coordination environment present in hemocyanin and tyrosinase.

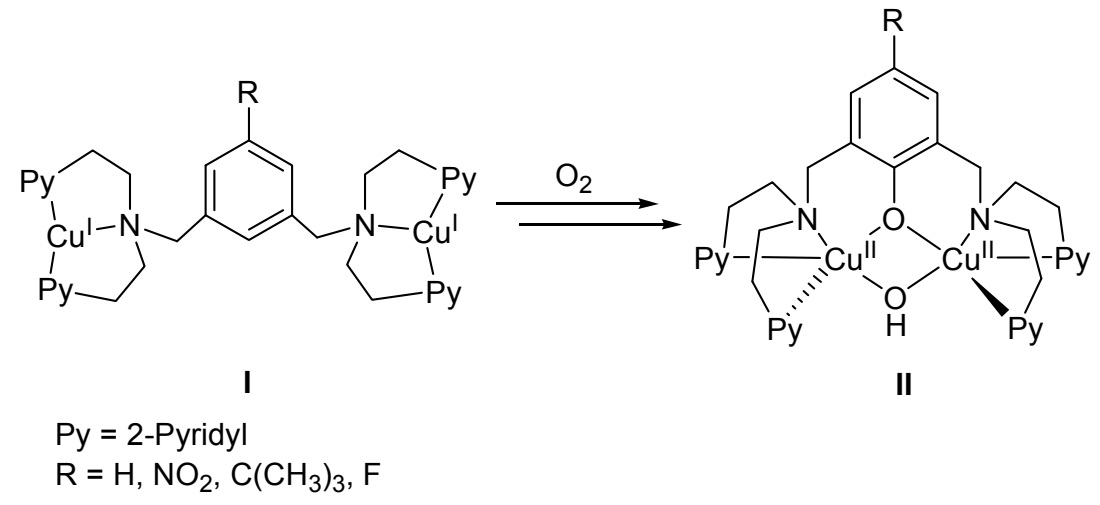

Scheme 4. Intramolecular hydroxylation of the phenyl group.

After addition of dioxygen to dicopper(I) complex I at low temperature, a dicopper(II)( $\mu$ $\eta^{2}: \eta^{2}$-peroxo) species was detected by UV/vis spectroscopy. Further reaction by electrophilic attack of the bridged peroxide to the phenyl spacer led to the formation of complex II. ${ }^{42,43}$ 
Shortly thereafter, Casella reported on a modification of the ligands employing $m$-xylyl spacer. This generation of ligands contained 1-methylbenzimidazole-groups as side arms. Formation of the stable dicopper(II) $\left(\mu-\eta^{2}: \eta^{2}\right.$-peroxo $)$ species $\mathbf{I V}$ only at $-80^{\circ} \mathrm{C}$ by reaction of complex III with dioxygen was proven by UV/vis and Raman spectroscopy, and the most interesting property of these species was the observation of tyrosinase activity (Scheme 5).

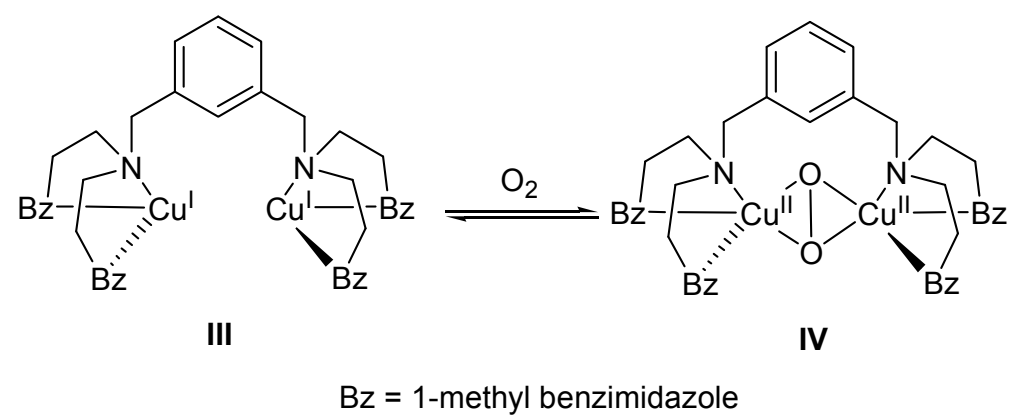

Scheme 5. The reversible oxygenation of the complex III, as reported by Casella.

The regiospecific ortho-hydroxylation of an exogenous, electron-poor phenol (4carbomethoxyphenol) to catechol and oxidation of an electron-rich catechol (3,5-di-tertbutylcatechol) to quinone was performed by III in the presence of dioxygen after formation of dicopper(II)( $\mu-\eta^{2}: \eta^{2}$-peroxo) complex IV at $-60^{\circ} \mathrm{C}$, as detected by $U V / v i s$ spectroscopy. ${ }^{44,45,46,47}$

The most important method for modulating the reaction behaviour of copper complexes is modification of their ligands, either by introducing different donor atoms, changing the chelate ring sizes, or using substituents that can influence the steric or electronic properties of the ligand.

A prominent class of ligands used for preparation of copper complexes is comprised of tripodal tetradentate $\left\{\mathrm{N}_{4}\right\}$ ligands (Figure 7). In general, copper(II) complexes of this type of ligands feature five-coordinate copper centers. Sterically restricting tripodal ligands, forming five-membered chelate rings, stabilize trigonal bipyramidal complexes. ${ }^{48,49,50}$ 

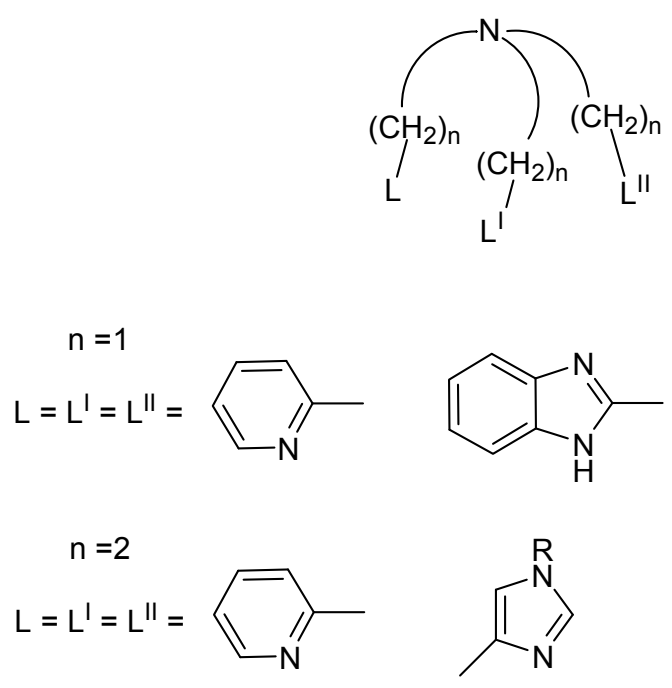<smiles>Cc1nc2ccccc2[nH]1</smiles><smiles>[R]c1cc([R])n(C)n1</smiles><smiles></smiles><smiles>[R]c1cc([R])n(C)n1</smiles>

Figure 7. Tripodal tetradentate nitrogen-based ligands reported in the literature.

When more flexible ligands are applied (with $n=2$, see Figure 7), six-membered chelate rings are formed preferentially, leading to a square pyramidal environment of the $\mathrm{Cu}$ ion. ${ }^{49,51,52} \mathrm{As}$ a result of their greater flexibility, ligands forming six-membered chelate rings when coordinated to $\mathrm{Cu}$ favor the stabilization of $\mathrm{Cu}^{\mathrm{I}}$ over $\mathrm{Cu}^{\mathrm{II}}$, relative to five-membered ring forming ligand systems.

Mononuclear complexes of tripodal tetradentate $\left\{\mathrm{N}_{4}\right\}$ ligands of the general form $\mathbf{V}$ (Figure 8) have proven highly successful for emulating the properties and reactivities of monometallic N-ligated metallobiosites. ${ }^{53}$ This is particularly true in biomimetic copper-dioxygen chemistry where manifold variations of the motif $\mathbf{V}$ have been employed, most prominently ligands of the tris(pyridylmethyl)amine type. ${ }^{54,55}$

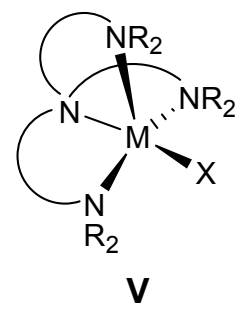

Figure 8. Schematic representation of the complex based on the tripodal tetradentate $\left\{\mathrm{N}_{4}\right\}$ ligand.

In order to mimic the active sites of tyrosinase and hemocyanin, tripodal ligands were modified using different kinds of spacers in-between the two tripodal units. It was found that 
different bridging groups could be introduced, e.g. using a xylyl linker between two tripodal ligands significantly stabilized the formation of the corresponding copper-peroxo complexes. Connecting the tripodal amines with two linkers leads to macrocyclic ligands. The potential of this approach has been highlighted by Bol with the preparation of a macrocyclic ligand Va where the tripodal units are connected through a pyrazolyl/ethyl bridge (Figure 9). ${ }^{56,57}$ With this ligand the dinuclear copper(I) complex forms an extremely stable peroxo complex after reaction with dioxygen. ${ }^{56}$

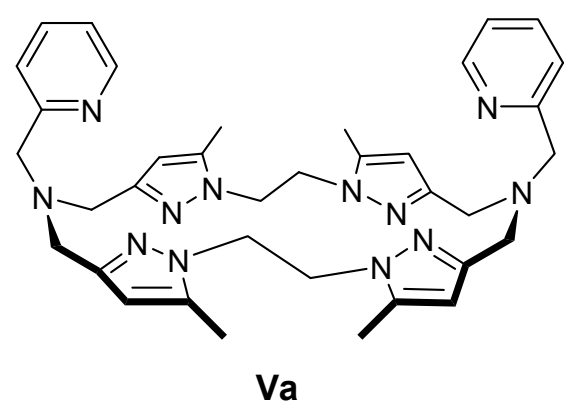

Figure 9. Schematic representation of the macrocyclic ligand Va with the tripodal units connected through a pyrazolyl/ethyl bridge.

These studies on $\mathrm{Cu}^{\mathrm{I}}$ and $\mathrm{Cu}^{\mathrm{II}}$ species with a range of similar ligands show that small changes in the ligand architecture can have a strong influence on the observed reactivity, in particular with $\mathrm{O}_{2}$. In the active sites of a variety of copper enzymes the nature of the ligand donors plays a pivotal role in forcing the metal ions to participate in the desired biochemical reactions. Thus further studies that focus on ligand modifications in synthetic complexes should provide deep insight into the fundamental principles of biologically relevant coordination chemistry. 


\section{Homogeneous copper catalyzed reactions}

\subsection{Hydroxylation and oxidation of phenols}

A lot of studies today focus not only on understanding the design rules in the biological systems involving Cu-dioxygen chemistry, but also on the factors governing the modes of reactivity observed with such systems as well as on the use of model bioinspired copper complexes for the catalytic oxidation or oxygenation of organic substrate molecules in homogeneous catalysis. ${ }^{58}$

As was already mentioned before, the selective ortho-hydroxylation of a phenolic substrate to yield the catechol derivative is catalyzed by the metalloenzyme tyrosinase. Few synthetic model systems exist that selectively perform the same monooxygenase reaction with exogenous substrates. ${ }^{59,60,61,62}$ The group of Casella has studied a series of dicopper complexes based on a $m$-xylyl bridgehead with 1-methylbenzimidazole side arms III, as well as an amine based asymmetric tripodal ligand VI (Figure 10). ${ }^{63}$

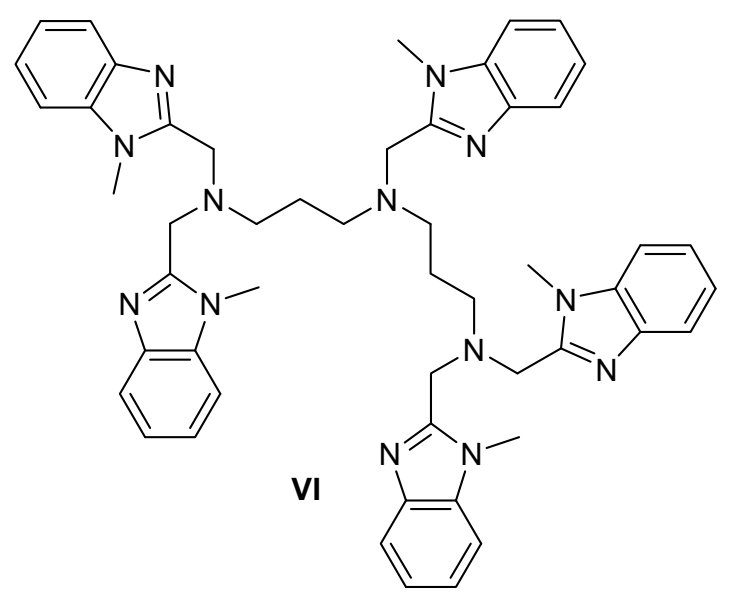

Figure 10. Asymmetric tripodal amine based ligand.

Methyl 4-hydroxybenzoate, as its tetra- $n$-butylammonium salt, was used to test the cresolase activity of two different dicopper(I) complexes, formed with the above-mentioned ligands (Scheme 6). Presence of the electron withdrawing ester-group leads to the stabilization of the dicopper(II)-catecholate product; thereby effectively increasing the selectivity of the catalytic reaction by preventing further redox and condensation reactivity of the product formed. 


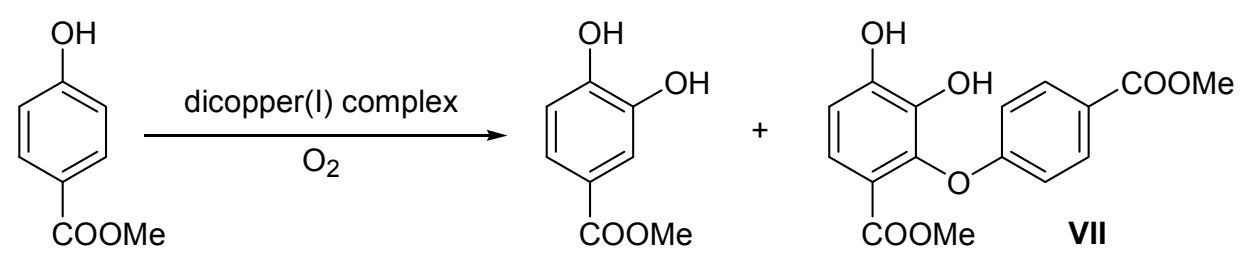

Scheme 6. Hydroxylation of the methyl 4-hydroxybenzoate.

Formation of VII was observed only in the case of complex III with the $m$-xylyl spacer, unless the reaction was carried out at low temperatures. Conversions were moderate however, with a maximum of $50 \%$. With the tripodal amine-based ligand VI no such undesired consecutive reactivity for the formed catechol was observed (i.e. no formation of methyl 2-[4(carbomethoxy)phenoxy]-3,4-dihydroxybenzoate VII), even at room temperature, and the product methyl 3,4-dihydroxybenzoate was formed as the sole product. Also with neutral 4hydroxybenzoic acid some reactivity was observed for the amine-based ligand.

A $\mu-\eta^{2}: \eta^{2}$-peroxo dicopper(II) complex based on the tridentate amine ligand VIII (Figure 11) was also found to act as a functional model for the cresolase activity of tyrosinase. Lithium salts of para-substituted phenols were used as substrates to react with the dicopper(II) peroxo complex in acetone at $-94^{\circ} \mathrm{C}$, reaching yields between 60 and $90 \%$ with only the catechol product formed. ${ }^{62}$ Isotope labeling experiments using ${ }^{18} \mathrm{O}_{2}$ confirmed that the origin of one of the $\mathrm{O}$ atoms of the catechol product is molecular oxygen.



Figure 11. Tridentate amine ligand.

$\mu-\eta^{2}: \eta^{2}$-Peroxodicopper(II) complex IX with a simple, bulky bidentate amine ligand was found to be active as well in the hydroxylation of phenolates at $-80{ }^{\circ} \mathrm{C}$ (in particular for 2,4-ditert-butylphenolate). Stack et al. were able to spectroscopically detect the formation of the bis( $\mu$-oxo)dicopper(III) phenolate complex $\mathbf{X}$ with a fully cleaved O-O bond (Scheme 7). ${ }^{64}$ These observations can be considered as an alternative mechanism for the catalytic hydroxylation of phenols, as carried out by the tyrosinase metalloenzyme. 


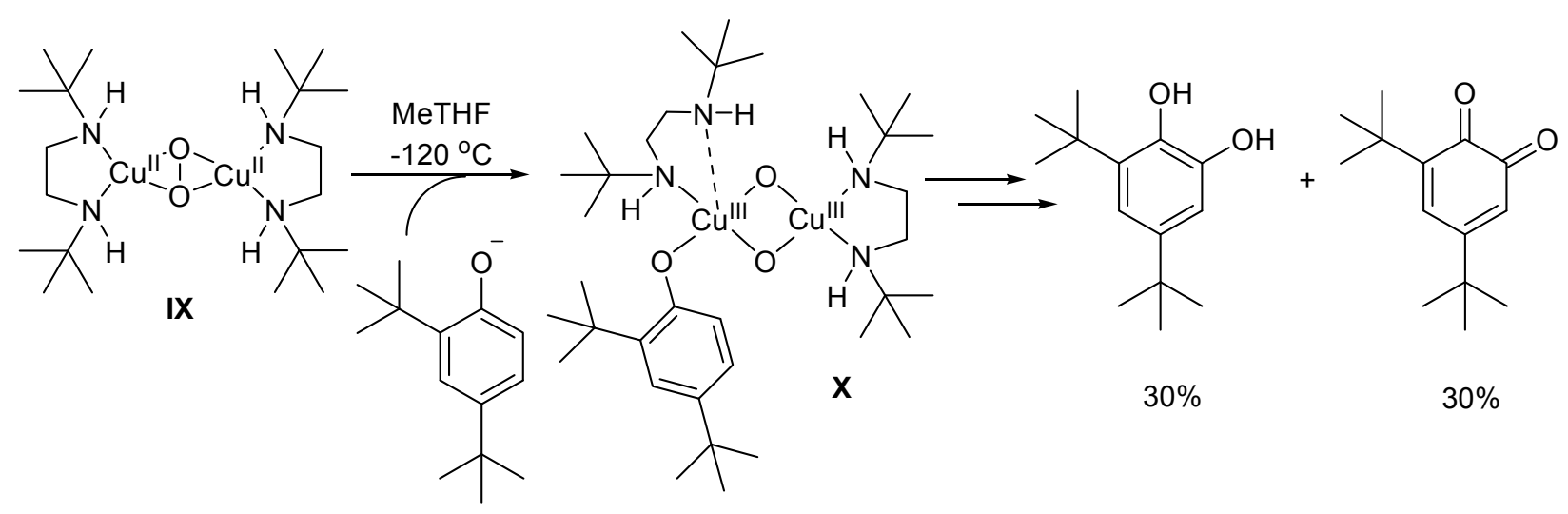

Scheme 7. Oxidation of 2,4-di-tert-butylphenolate to the corresponding catechol and quinone.

Catechols formed after ortho-hydroxylation of a phenolic substrate can be further transformed into the corresponding $o$-quinones. This selective oxidation reaction is catalyzed by both tyrosinase and catechol oxidase. Various mono- and dinuclear copper coordination compounds have been investigated as biomimetic catalysts for the catechol oxidation, ${ }^{11,65}$ in most cases using 3,5-di-tert-butylcatechol (DTBC) as the model substrate (Scheme 8). The bulky tert-butyl groups prevent undesirable side reactions such as polymerization of the resulting quinone to yield brown melanin. Depending on the copper catalyst used, either $\mathrm{H}_{2} \mathrm{O}_{2}$ or $\mathrm{H}_{2} \mathrm{O}$ may be formed as the reduction product.

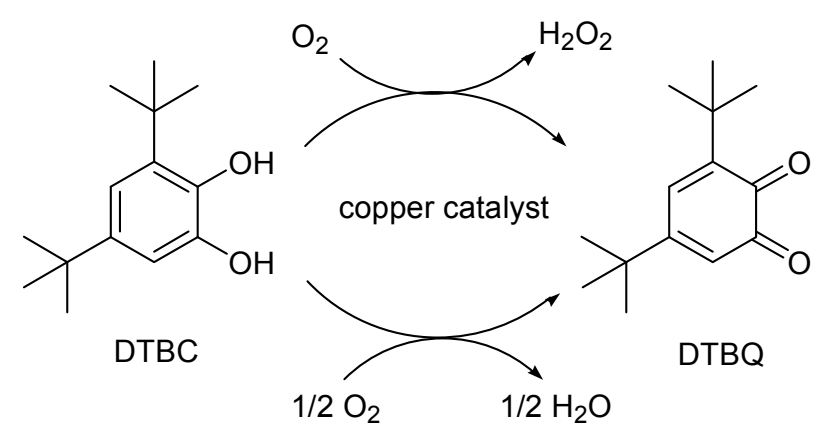

Scheme 8. Oxidation of DTBC by copper catalyst.

Casella and co-workers were successful in determining the reaction rates for the two proposed steps of the catalytic cycle (fast binding of catechol to the dicopper(II) complex and slow oxygenation of the dicopper(I) species with a further binding of a second catechol to the dicopper(II) peroxo intermediate, followed by electron transfer from the catechol anion to the dicopper intermediate), but detailed mechanistic studies are still under debate. ${ }^{66,67,68,69,70}$ 
It was found that dinuclear copper complexes are more reactive than mononuclear compounds in this particular kind of oxidation catalysis. ${ }^{71,72,73}$ The activity of synthetic dicopper model complexes can be tuned by different parameters, such as the intramolecular metal-metal separation, ${ }^{71,74,75,76}$ the redox properties of the two $\mathrm{Cu}$ ions (in a fine interplay with the properties of the ligand donor-groups), ${ }^{74,77,78}$ and by the structure of the dinucleating ligands. ${ }^{71,78,79,80}$ For example, well-known dicopper complexes derived from pyrazolate-based dinucleating ligands that differ only by the length of the chelate side arms (which allows to control the metal-metal distance), the number of donor atoms (which determines the number of accessible coordination sites), and the type of donor atom (e.g. aromatic versus aliphatic N, which changes the redox properties) ${ }^{71,81}$ (Scheme 9) were found to be active in the oxidation of catechols to the corresponding quinones.

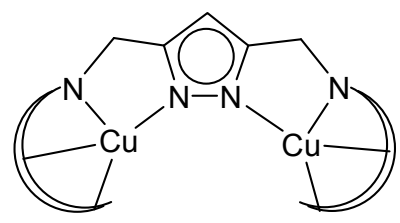

chelate side arms
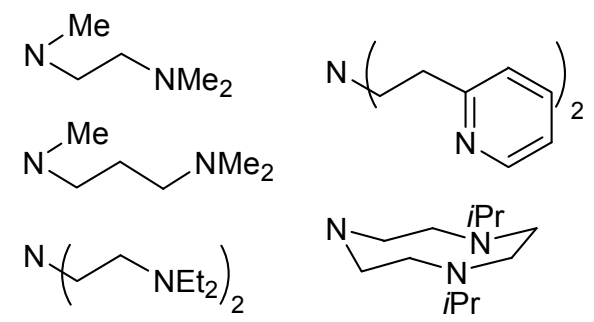

Scheme 9. Schematic representation of a family of dinucleating compartmental pyrazole ligands and the general dicopper(II) complex derived from them.

Such kind of ligand modification leads to a distinct activity in the catalytic oxidation of DTBC mediated by those dicopper complexes. ${ }^{71,74}$ One of the important factors that determine the catalytic activity of these complexes is the $\mathrm{Cu} \cdots \mathrm{Cu}$ distance. The shortest accessible separation with the available set of systems $(\sim 3.50 \AA)$ is clearly advantageous for high activity. 


\subsection{Oxidative polymerization and coupling of phenols}

Several bioinspired model copper complexes were also found to be good catalysts for the oxidative polymerization and coupling of various phenols. It was first discovered in 1959 that a $\mathrm{Cu}^{\mathrm{I}}$-pyridine complex, in the presence of dioxygen as an oxidant, was able to catalyze the oxidative coupling of phenols. Many studies were carried out to optimize the reaction conditions, in particular the factors that influence the reactivity and selectivity of the product formation. It was established that the presence of two small ortho-groups on the phenol ring guides the reaction in the direction of polymerization. In the meantime, larger substituents shift the selectivity towards the formation of undesirable diphenoquinone (DPQ) during the polymerization. ${ }^{82}$ Considering these results, 2,6-dimethylphenol was chosen as a monomer, to yield poly(2,6-dimethylphenylene ether) (PPE) as the product after polymerization (Scheme $10)$.

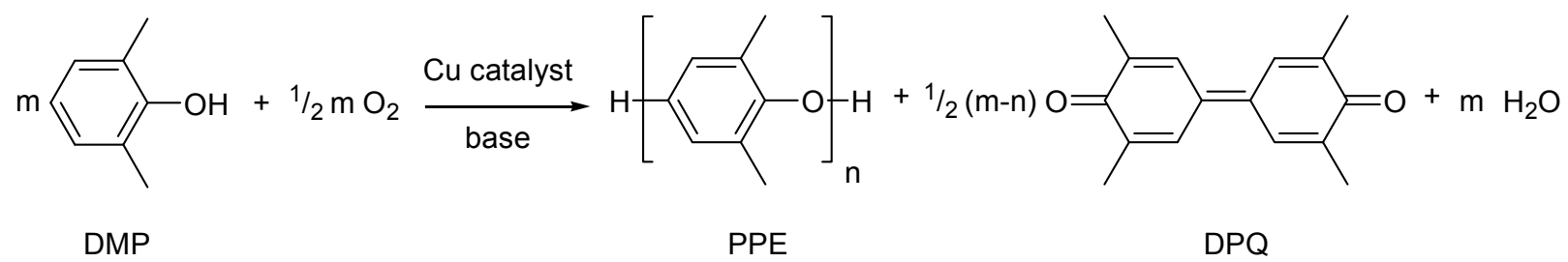

Scheme 10. Copper catalyzed oxidative polymerization of DMP.

PPE is an important ingredient of high-performance engineering plastics. It is a thermoplastic polymer that exhibits unusually low moisture absorption, leading to good electrical insulating properties over a wide humidity- and temperature range. It is also very resistant to a variety of chemicals, water, most salt solutions, acids and bases. Main applications for this material include computer and television housing, keyboard frames and interface boxes.

This polymerization reaction has been extensively studied but the mechanism has not yet been well clarified. ${ }^{83}$ Reedijk and co-workers were able to selectively obtain PPE with a molecular weight $M_{\mathrm{w}}$ of up to $\sim 86000 \mathrm{Da}$, using a biphasic toluene/water emulsion, with an in situ prepared $\mathrm{Cu}(\mathrm{MeIm})$-catalyst $(\mathrm{MeIm}=1$-methylimidazole $)$. The catalytic results were highly dependent on the ligand:metal ratio used. ${ }^{84}$ Different imidazole derivatives were used as ligands and it was found that monodentate ligands led to more active catalysts in comparison 
to bidentate derivatives. Also, it was shown that the activity of the copper catalyst increased with higher ligand basicity. ${ }^{83}$

The oxidative polymerization of DMP could also be carried out in aqueous sodium hydroxide solution $(\mathrm{pH} \sim 13.5)$ at $50{ }^{\circ} \mathrm{C}$ under vigorous stirring using (tmeda) $\mathrm{CuCl}_{2}$ (where tmeda is $N, N, N^{\prime}, N^{\prime}$-tetramethylethylenediamine) as catalyst. In this case only traces of the undesired product DPQ were observed and polymeric material with a $M_{\mathrm{n}}$ of $2900 \mathrm{~g} / \mathrm{mol}$ was obtained as the main product. $^{85}$

Reactions involving the oxidative coupling of phenols are of great importance for general organic synthesis as well as for the production of pharmaceutical chemicals. The classic example of copper catalyzed coupling is the Ullmann biaryl coupling where a phenol and arylhalide substrate are reacted to yield a diarylether (Scheme 11). ${ }^{86,87}$

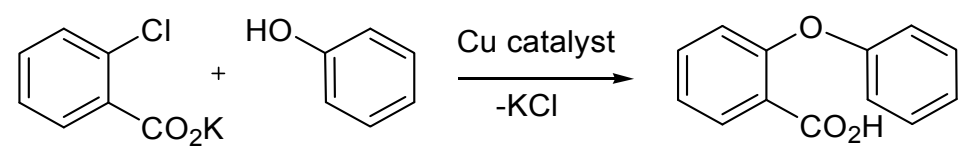

Scheme 11. Ullmann coupling of phenol.

The oxidative C-C coupling of phenols with bulky groups, e.g. tert-butyl substituents, can also be catalyzed by dicopper(I) complexes. Thus, the dicopper(I) complex formed after coordination of the dinucleating bisamino-bispyridine ligand XI was shown to couple 2,4-ditert-butylphenol in the ortho-position to the corresponding bisphenol derivative (Scheme 12).
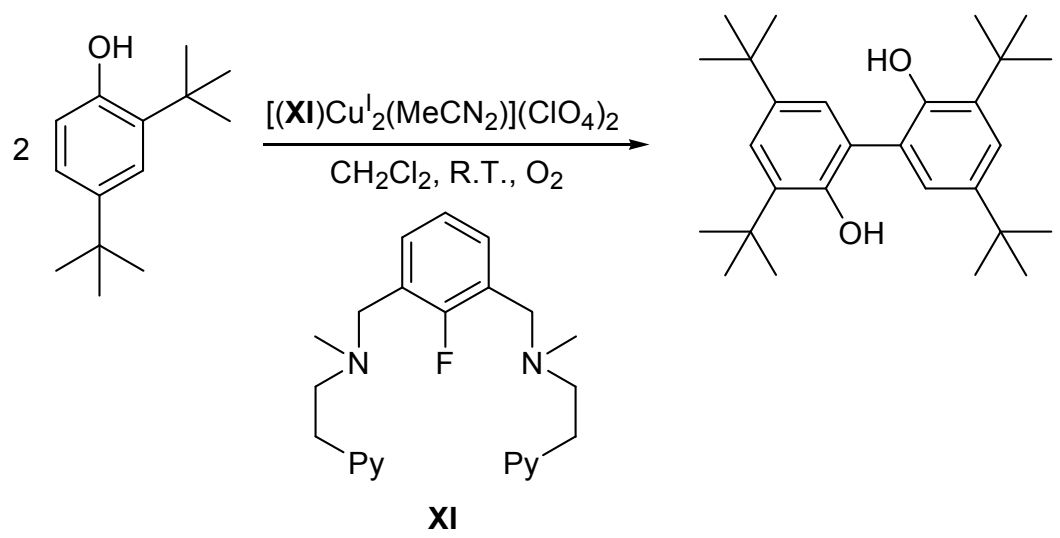

Scheme 12. C-C coupling of 2,4-di-tert-butylphenol. 
At the same time, use of phenolic substrates without substitution at the para-position led to oxidative coupling in the para-position, resulting in the formation of diphenoquinone derivatives (e.g. in case of 2,6-dimethylphenol, DPQ is formed as the main product). ${ }^{88}$ Similar reactivity is observed for various other dicopper systems. ${ }^{89}$

\subsection{C-H bond activation}

Oxidation of alkanes and alkenes under aerobic conditions is of great importance from both a bioinorganic and a synthetic organic point of view. ${ }^{90}$ Nowadays the selective functionalization of, inter alia, the family of alkanes via $\mathrm{C}-\mathrm{H}$ bond activation receives much attention, as this would open up new feedstocks and routes for organic synthesis for laboratory and industrial scale applications.

It was found by Itoh and co-workers that $\beta$-diketiminato ligands with the general formula XII (Figure 12) were able to form complexes with copper(I) or copper(II) ions, yielding the selective formation of different species, depending on the specific conditions employed. ${ }^{91}$ $\operatorname{Bis}(\mu-\mathrm{oxo}) \mathrm{Cu}_{2}{ }_{2}$ si species were obtained after reaction of either $\mathrm{Cu}^{\mathrm{I}}$ and $\mathrm{Cu}^{\mathrm{II}}$ complexes with $\mathrm{O}_{2}$ and $\mathrm{H}_{2} \mathrm{O}_{2}$, respectively. In addition to the formation of such copper(III)-oxo species, the copper(II) complexes were also employed as pre-catalysts for the oxidation of alkanes (cyclohexane and adamantane) in the presence of $\mathrm{H}_{2} \mathrm{O}_{2}$. These complexes have therefore been described as functional models for pMMO (particulate methane monooxygenase). ${ }^{92}$

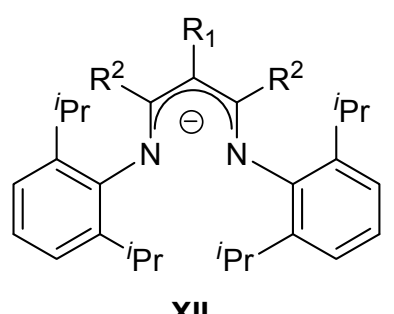

XII

Figure 12. Schemetic representation of $\beta$-diketiminato ligands used by Itoh.

Copper-containing mono-oxygenases such as peptidylglycine- $\alpha$-hydroxylating monoxygenase (PHM) catalyze the oxidation of aliphatic $\mathrm{C}-\mathrm{H}$ bonds. Recent studies on the reactivity observed with model complexes for this particular type of metalloenzymes were carried out by Murahashi and co-workers, who have reported that with $\mathrm{CuCl}_{2}$ and acetaldehyde and in the 
presence of 18-crown-6, cyclohexane could be oxidized to the corresponding cyclohexanone under relatively mild conditions $\left(70{ }^{\circ} \mathrm{C}\right)$. Cyclohexanol was formed as the major side product. ${ }^{93}$ The achieved yield of cyclohexanone was $61 \%$, with turnover numbers of up to 1600 (Scheme 13).

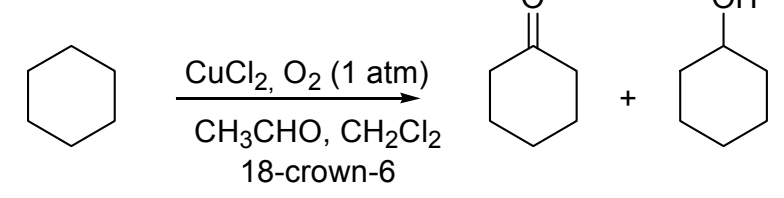

Scheme 13. Oxidation of cyclohexane.

It was recently reported that reaction of $\mathrm{Cu}\left(\mathrm{NO}_{3}\right)_{2}$ with triethanolamine in the presence of sodium hydroxide and different types of aromatic carboxylates, and $\mathrm{NaN}_{3}$ or $\mathrm{NaBF}_{4}$ as additional reagent, yielded multinuclear copper(II) complexes with different structural characteristics, e.g. trinuclear complex XIII (Figure 13). These complexes were found to be active systems for the copper catalyzed oxidation of cyclohexane in $\mathrm{MeCN}$, using $\mathrm{H}_{2} \mathrm{O}_{2}$ as oxidant. $^{94}$

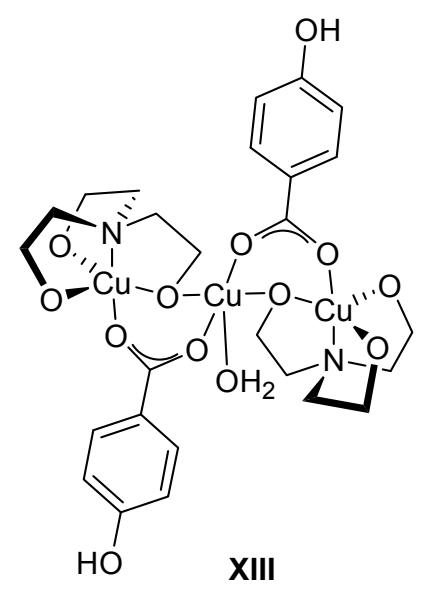

Figure 13. Trinuclear copper complex used for the oxidation of cyclohexane.

The optimal molar ratio of peroxide to catalyst was found to be in the range from 200:1 to 400:1 with total conversions (cyclohexanol and cyclohexanone combined) of up to $32 \%$ after $72 \mathrm{~h}$ at room temperature. 
Moreover, copper(I) systems based on various N-donor ligands such as pyridine, bipyridine, tris(pyrazolyl)borate (tpb), and tris(pyrazolyl)methane (tpm) have been reported as catalysts for the oxidation of alkanes, alkenes, and alcohols using $t \mathrm{BuOOH}$ and 1 atm of dioxygen pressure. These mononuclear copper(I) complexes were inactive with regard to oxygen activation. However, reaction of these complexes with $t \mathrm{BuOOH}$ produced an intermediate $\mathbf{X I V}$, which reacted further to form the superoxide species [ $\left.\mathrm{LCu}^{\mathrm{II}}(\mathrm{OO} t \mathrm{Bu})(\mathrm{OO})\right]$ (Scheme 14). ${ }^{95}$

$$
\begin{aligned}
& \mathrm{L}(\mathrm{py}) \mathrm{Cu} \mathrm{u}^{\prime}+t \mathrm{BuOOH} \longrightarrow\left[\left(\mathrm{LCu} \mathrm{OOOtBu}^{\prime}+\mathrm{pyH}^{+}\right]\right. \text {XIV } \\
& {\left[\left(\mathrm{LCu}^{\prime} \mathrm{OO} \mathrm{BBu}\right)+\mathrm{pyH}^{+}\right] \stackrel{\mathrm{O}_{2}}{\longrightarrow}\left[\left(\mathrm{LCu}^{\prime \prime}(\mathrm{OO})(\mathrm{OOtBu})\right)+\mathrm{pyH}^{+}\right]}
\end{aligned}
$$

Scheme 14. Formation of the superoxide species from mononuclear pyridine base copper(I) complex.

Reaction of $1 \mathrm{M}$ ethylbenzene under 1 atm of $\mathrm{O}_{2}$, and in the presence of copper(I)(tpb) complex and $t \mathrm{BuOOH}$ led to the formation of acetophenone as basically the only product in almost $60 \%$ conversion.

The catalytic oxidation of benzylic $\mathrm{C}-\mathrm{H}$ bonds is also feasible in the presence of copper complexes. One of the frequently used model substrates to probe the catalytic activity of a given $\mathrm{Cu}$-complex in the oxidation of benzylic $\mathrm{C}-\mathrm{H}$ bonds is 2,4,6-dimethylphenol (TMP), which can not undergo C-O polymerization.

Combination of $\mathrm{CuCl}_{2}$ in the presence of amine or oxime ligands and alcohol (in particular $\mathrm{MeOH})^{96}$ leads to the formation of copper systems that show activity in the selective oxidation of the para-methyl group by molecular oxygen to yield 3,5-dimethyl-4hydroxybenzaldehyde XVII (Scheme 15). This transformation bears some similarity to the oxygenation of aromatic $\mathrm{CH}_{3}$-groups to the corresponding aldehyde, as catalyzed by laccase or vanillyl alcohol oxidase. ${ }^{97}$

Reedijk and co-workers recently reported another copper system that is able to catalyze this kind of benzylic C-H oxidation. ${ }^{98}$ Since the oxidation of TMP to a corresponding aldehyde is an overall four-electron process, four equivalents of a $\mathrm{Cu}^{\text {II }}$-based system $\left[\mathrm{CuCl}_{2}(\mathrm{neo}) / \mathrm{NaOMe}\right]$ (neo $=2,9$-dimethylphenanthroline) were used to stoichiometrically 
oxidize TMP selectively to 3,5-dimethyl-4-hydroxybenzaldehyde (XVII) at room temperature in $\mathrm{MeOH}$. This reaction could also be conducted in a catalytic fashion, using $\mathrm{H}_{2} \mathrm{O}_{2}$ as oxidant in refluxing $\mathrm{MeOH}$.

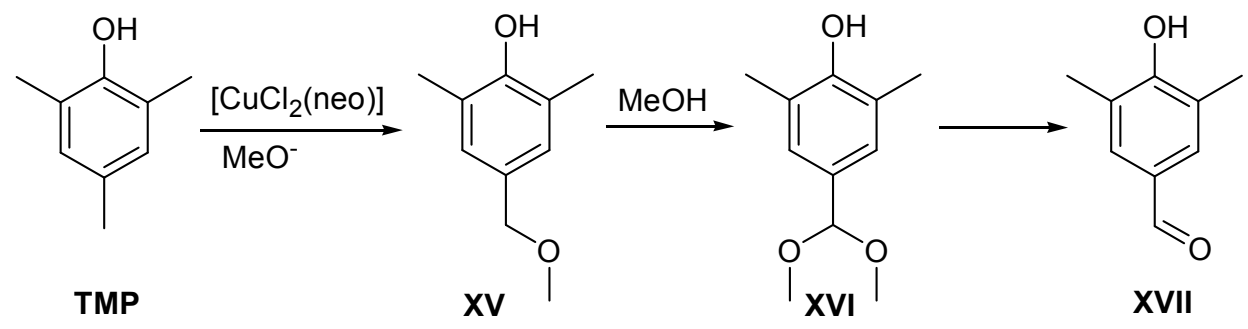

Scheme 15. Sequential oxidation of TMP to XVII, catalyzed by the $\left[\mathrm{CuCl}_{2} / \mathrm{neo} / \mathrm{NaOMe}\right]$ system.

A decrease in the stoichiometry NaOMe:TMP led to selective isolation of the intermediate product 4-(methoxymethyl)-2,6-dimethylphenol XV (MDP). However, the catalytic activity of the $\mathrm{CuCl}_{2}$ (neocuproine) system is rather low with a turnover frequency of $\sim 1.4 \mathrm{~h}^{-1}$. It has been proposed that the reaction proceeds through formation of a $\mathrm{Cu}$-bound phenoxyl radical (that reacts with $\mathrm{O}_{2}$ ) and a benzoquinone methide intermediate, followed by repeated 1,6addition of alcohol to sequentially form 4-(methoxymethyl)-2,6-dimethylphenol XV and 3,5dimethyl-4-hydroxybenzaldehyde dialkyl acetal XVI, which is further hydrolyzed to yield 3,5-dimethyl-4-hydroxybenzaldehyde XVII.

In order to get some insight into a possible reaction pathway or active $\mathrm{Cu}$-species involved in the observed catalysis, pentafluorophenol $\left(\mathrm{C}_{6} \mathrm{~F}_{5} \mathrm{OH}\right)$ was applied as a model substrate. Together with the ligand (neo) and in the presence of $\mathrm{NaOMe}$ and $\mathrm{CuCl}_{2}$, formation of a dinuclear copper(II) core, bridged by a methoxide and a phenoxide, was observed (Figure 14).

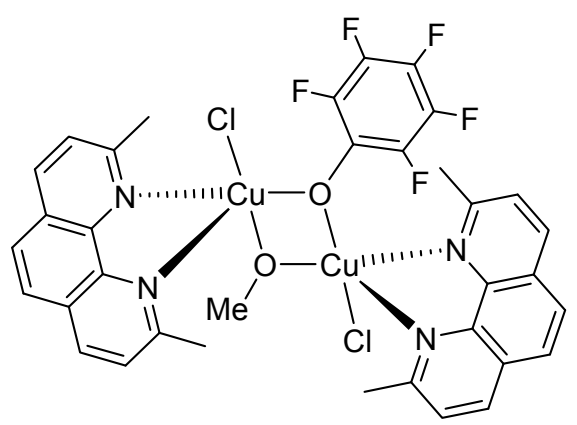

Figure 14. Model dinuclear copper(II) complex with a methoxide and a phenoxide bridge. 
The $\left[\mathrm{CuCl}_{2}\right.$ (neocuproine)/NaOMe] system mentioned above has also been reported as a catalyst for the 1,6-nucleophilic addition of either 1,2-ethanediol, 2,2'-dipyridylamine or 2,4pentanedione to TMP. These oxidative coupling reactions of TMP with various nucleophiles proposedly proceed via the in situ formation of a benzoquinone methide. ${ }^{99}$ Reactions were carried out in DMF as non-nucleophilic solvent, and with $\mathrm{NaOMe}$ as base to deprotonate the para $\mathrm{CH}_{3}$-group of TMP to form the proposed reactive benzoquinone methide intermediate. No oxidative coupling was observed without neocuproine present in the system, which can be considered as evidence for the necessary stabilization of the $\mathrm{Cu}^{\mathrm{I}}$-oxidation state with a bulky bidentate ligand. It was assumed that reduction of $\mathrm{Cu}^{\mathrm{II}}$ to $\mathrm{Cu}^{\mathrm{I}}$ takes place together with formation of the benzoquinone methide fragment. 


\subsection{Goals of the work}

The framework of the thesis is provided by newly developed pyrazole-based ligands with bioinspired chelating side arms in the 3- and 5-positions of the heterocyclic core. The synthesis of these compounds will be described and they will be applied as valuable scaffolds for further studies. The design-concept for these ligands makes use of the fact that the anionic pyrazolate unit has a high tendency to span two metal ions in a $\mu_{-}-\eta^{1}: \eta^{1}$ exo-bidentate fashion, while appropriate modification of the side-arms of such ligand systems makes it possible to tune the properties of the copper complexes derived from them. The coordination spheres of the individual copper ions as well as the intramolecular metal-metal separation are the most important factors when synthesizing models for biologically relevant dicopper complexes; for pyrazolate-bridged complexes, these parameters can be altered by appropriate modifications of the appended chelate substituents.

New bioinspired dinucleating ligand scaffolds that have binding compartments composed of imidazolyl groups will be reported. These ligands form very stable copper complexes, and initial studies will be performed to determine the different species formed in solution, depending on the $\mathrm{pH}$ (in particular for complexes based on a ligand with appended bis[2-(1methylimidazolyl)methyl]aminomethyl chelate arms).

It is necessary to obtain a comprehensive picture of the copper coordination chemistry of the new ligand systems for any further (reactivity) studies to be performed. This will require a full characterization of the various copper complexes obtained, both in solution and in the solid state (e.g. by spectroscopic methods and X-ray crystallography).

Properly characterized copper complexes with carefully tuned and desired properties (nature of the chelate arms, metal-metal separation, nuclearity) will be further applied as catalysts in different types of oxidation of phenolic substrates. In particular, attention will be given to the oxidative C-C coupling of 2,6-dimethylphenol (DMP) and 2,4,6-trimethylphenol (TMP), as well as to the 1,6-nucleophilic addition of several small molecules to TMP, such as MeOH. In order to gain mechanistic insight, specifically for the oxidative C-C coupling of TMP, the reactions (both catalytic and stoichiometric with regard to the phenols used) will be monitored with a variety of spectroscopic methods and organic intermediates formed during the reaction will be identified. 
Moreover, to shed light on the formation and structure of possible intermediates in the catalytic reactions studied, the coordination chemistry of these dicopper complexes with phenolic substrates that display no $\mathrm{Cu}$-mediated transformation will be a subject of research.

All these above-mentioned studies have to provide a better understanding of the principles/factors that govern the catalytic oxidation activity of pyrazolate-based bimetallic model complexes towards phenols. Various kinds of correlations (e.g. metal-metal separation and effective cooperativity of two adjacent copper ions; behaviour of new copper complexes in solution and catalytic activity in the $\mathrm{C}-\mathrm{H}$ bond activation) will be discussed.

Furthermore, some specific examples of unprecedented C-C and C-O bond forming reactions with 4-ethylphenol and 4-bromo-2,6-dimetylphenol are described. 


\section{$4 \quad$ Ligand synthesis}

\subsection{Properties of pyrazole-based compartmental ligands}

As was already described before, tripodal tetradentate $\left\{\mathrm{N}_{4}\right\}$ ligands (such as the tris(pyridylmethyl)amine type $)^{54,55}$ were sucsesfully applied to emulate the properties and reactivities of mononuclear N-ligated metalloenzymes. From this point of view, the pyrazolebridging ligands used in the present work can be described as two tripodal tetradentate $\left\{\mathrm{N}_{4}\right\}$ subunits XVIII bridged by a heterocyclic pyrazole core to give the overall ligand system XIX (Scheme 16). This general ligand architecture allows the direct and selective formation of dinuclear complexes with highly tunable properties (e.g. metal-metal separations can range from 3.40 to $4.50 \AA)^{13,15,16}$

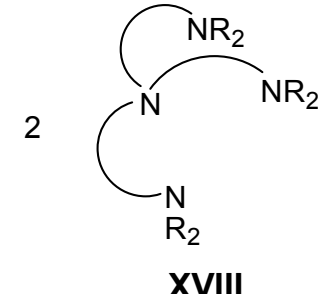

XVIII



XIX

Scheme 16. Schematic representation of the $\left\{\mathrm{N}_{4}\right\}$ subunit XVIII and pyrazole-bridging ligand XIX.

The set of pyrazole-based ligands synthesized and used in this work is depicted in Figure 15. $\mathbf{H L}^{1}$ and $\mathbf{H L}^{2}$ have been reported previously ${ }^{71}$ and represent pyrazole-based ligands with aliphatic side arms. This pair of ligands differs only by the number of additional N-donorfunctionalities, which allows for the manipulation of the geometry of the copper ions in the complexes as well as the redox properties of the dicopper core. Presence of the pyridine-Ndonor compartments in ligand $\mathbf{H L}^{3}$ provides for additional metal-to-ligand backbonding, which leads to a change in the redox properties of the copper ions. Such kind of electronic fine-tuning is not possible for ligands with aliphatic compartments.

The last two ligands $\mathbf{H L}^{4}$ and $\mathbf{H L}^{\mathbf{5}}$ have binding compartments composed of imidazolyl groups. These new pyrazole-based ligands have been designed and prepared with the aim of further advancing the emulation of biological donor environments of copper ions in relevant 
model complexes. The pendant imidazole donors in $\mathbf{H L}^{4}$ and $\mathbf{H L}^{\mathbf{5}}$ significantly increase the biomimetic character of these ligands, and it is foreseen that these systems can be very interesting as building blocks for the synthesis of copper complexes that may exhibit promising catalytic properties. All ligands used were prepared via multi-step procedures.

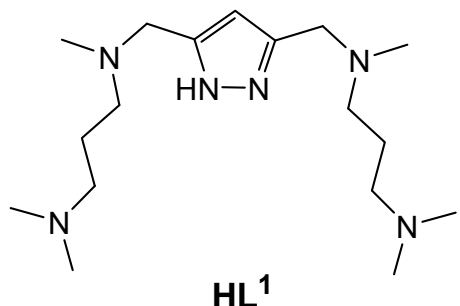

$H L^{1}$

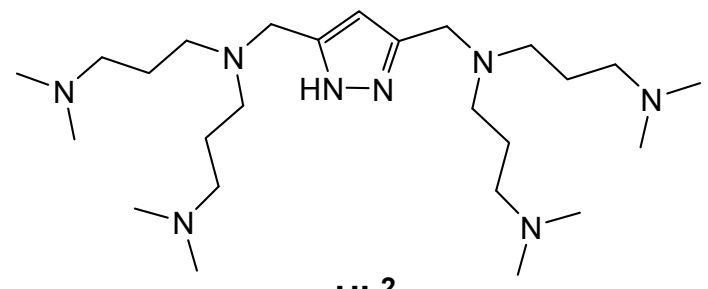

$\mathrm{HL}^{2}$<smiles>Cc1cccc(CN(Cc2cc(CN(Cc3cccc(C)n3)Cc3cccc(C)n3)[nH]n2)Cc2cccc(C)n2)n1</smiles>

$H L^{3}$<smiles>Cn1ccnc1CN(Cc1cc(CN(Cc2nccn2C)Cc2nccn2C)[nH]n1)Cc1nccn1C</smiles>

$\mathrm{HL}^{4}$<smiles>Cn1ccnc1CN(Cc1n[nH]c(CN(Cc2nccn2C)Cc2nccn2C)c1-c1ccccc1)Cc1nccn1C</smiles>

$\mathrm{HL}^{5}$

Figure 15. Pyrazole-based ligands $\mathbf{H L}^{1}-\mathbf{H L}^{5}$. 


\subsection{Synthesis of ligands}

The herein described bis(aminomethyl)-substituted pyrazole-based ligands can be synthesized using the following procedure depicted in Schemes 10 and 11. At first, 3,5-bis(chloromethyl)1-(tetrahydropyran-2-yl)pyrazole XXV has to be prepared according to the literature (Scheme 17). ${ }^{100,101,102}$ By oxidation of 3,5-dimethylpyrazole $\mathbf{X X}$, the mono potassium salt of 3,5pyrazole dicarbonic acid XXI can be obtained. Further esterification of XXI is performed under acidic conditions in methanol. Alcohol XXIII is obtained upon reduction of ester XXII in the presence of $\mathrm{LiAlH}_{4}$ in diethylether, and this former compound can thereafter be chlorinated using thionyl chloride, which at the same time serves as the solvent for this reaction, to yield 3,5-bis(chloromethyl)pyrazole XXIV. The last step in the preparation of 3,5bis(chloromethyl)-1-(tetrahydropyran-2-yl)pyrazole $\mathbf{X X V}$ is protection of the NH-group of the pyrazole by 3,4-dihydro-2H-pyran in dichloromethane (DHP).
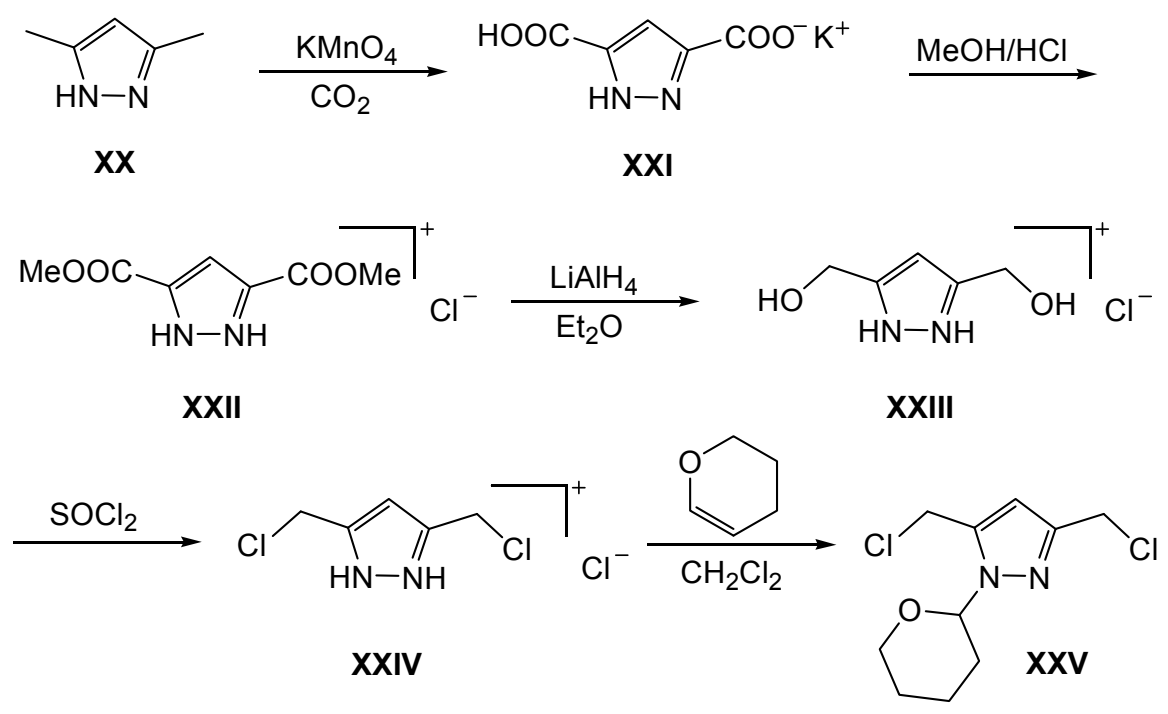

Scheme 17. Preparation of the 3,5-bis(chloromethyl)-1-(tetrahydropyran-2-yl)pyrazole XXV.

It has to be mentioned that protection of the pyrazole NH-functionality with DHP is not always a necessary step for the preparation of amine-based functional derivatives of pyrazoles. Depending on the amine used for the coupling with the bis(chloromethyl) derivative, the above-mentioned protection step can be excluded, and 3,5bis(chloromethyl)pyrazole XXIV can directly undergo reaction with amines to yield the desired ligand with general structure XXVI (Scheme 18). This direct route works especially well for aliphatic and some aromatic amines. 


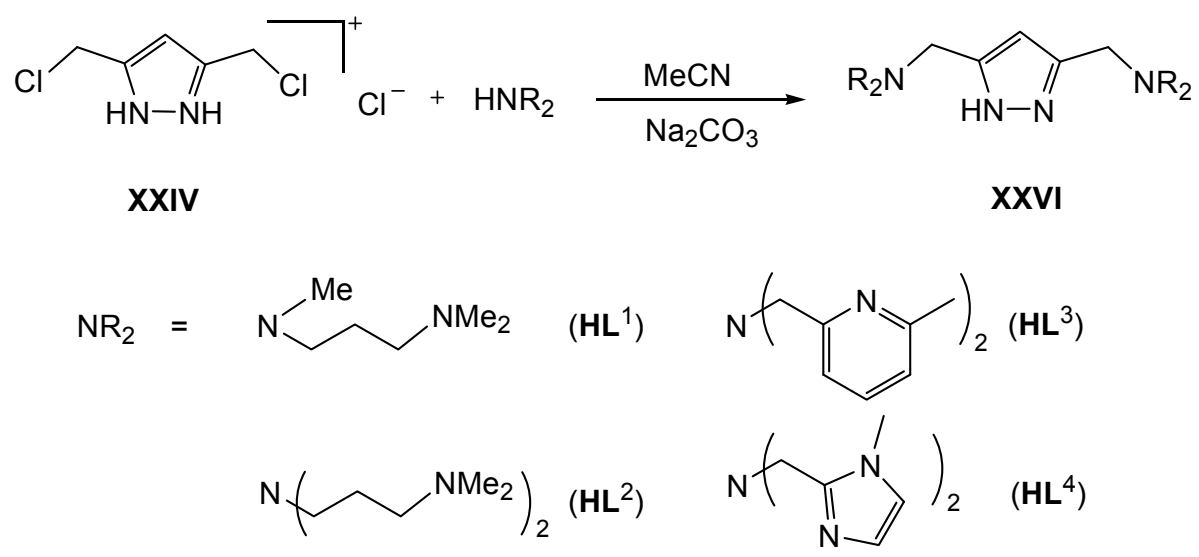

Scheme 18. Synthesis of ligands with aliphatic and aromatic side arms.

This advantage (excluding the NH-protection reaction) was applied in the synthesis of all ligands used, which increased their yields, as especially the deprotection of the pyrazole NHunit is known to lead, in general, to significant product loss.

The amines used for the synthesis of ligands $\mathbf{H L}^{3}, \mathbf{H L}^{4}$ and $\mathbf{H L}^{5}$ were prepared in multistep syntheses following (modified) literature procedures. ${ }^{103}$

To obtain bis[(6-methyl-2-pyridyl)methyl]amine $\quad$ XXIX, ${ }^{103 a} \quad 6$-methylpyridine-2carboxaldehyde XXVII was reacted with hydroxylamine hydrochloride, yielding 6methylpyridine-2-oxime XXVIII. The last step in the preparation of this desired amine is hydrogenation of the oxime in a mixture of methanol/water (Scheme 19).

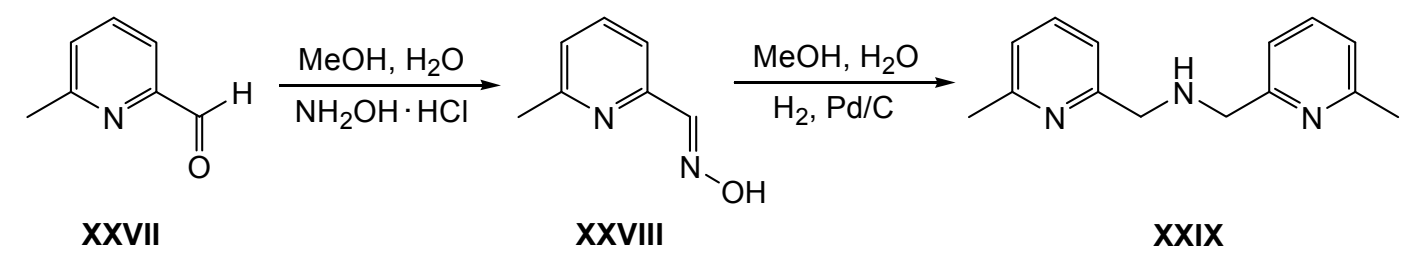

Scheme 19. Preparation of bis[(6-methyl-2-pyridyl)methyl]amine.

Bis[(1-methylimidazol-2-yl)-methyl]amine XXXIII was prepared via a four step synthetic route, ${ }^{103 b, c}$ starting from $N$-methylimidazole $\mathbf{X X X}$, which was deprotonated by $n$-BuLi and then reacted with DMF, yielding $N$-methylimidazolyl-2-aldehyde XXXI. Preparation of $N$ methylimidazolyl-2-oxime XXXII was carried out in an analogous way as for XXIX, in the presence of hydroxylamine hydrochloride. Hydrogenation of the corresponding oxime was performed on $\mathrm{Pd} / \mathrm{C}$ catalyst, yielding product XXXIII (Scheme 20). 

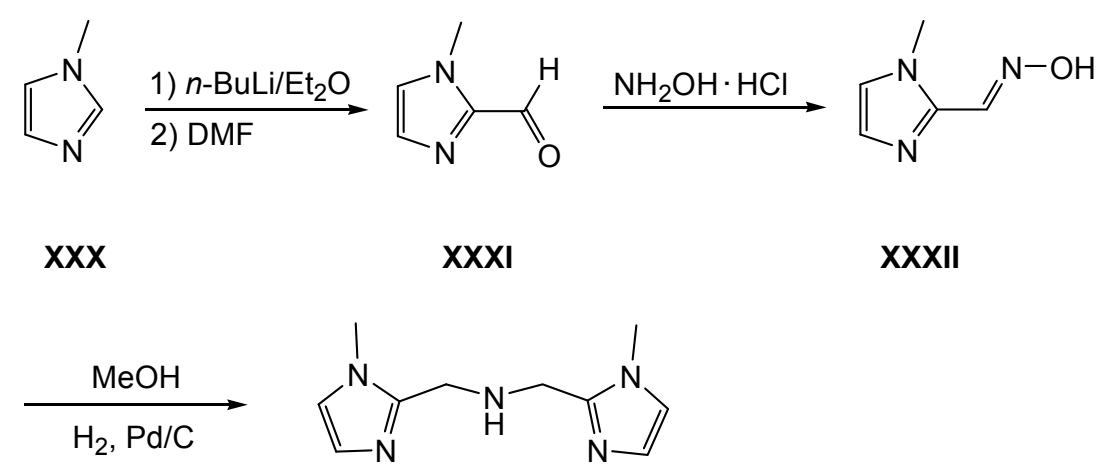

XXXIII

Scheme 20. Synthesis of bis[(1-methylimidazole-2-yl)-methyl $]$ amine.

Inclusion of a phenyl group into the backbone of the central pyrazole unit, effectively yielding 3,5-bis(hydroxymethyl)-4-phenyl-pyrazole XXXVIII, requires drastic changes in the synthetic procedure to obtain this starting material. In this case, commercially available glycine methylester hydrochloride XXXIV was reacted with sodium nitrite in the presence of sulfuric acid, yielding diazoacetic acid methylester XXXV as orange oil. ${ }^{104}$ Subsequently, XXXV was reacted with methylcinnamate to obtain the methylester of 4-phenylpyrazoline dicarbonic acid XXXVI ${ }^{105}$ which could be oxidized by bromine to the corresponding pyrazole analogue XXXVII. The end-product XXXVIII was cleanly obtained upon reduction of XXXVII with $\mathrm{LiAlH}_{4}$ in $\mathrm{Et}_{2} \mathrm{O}$ (Scheme 21). 


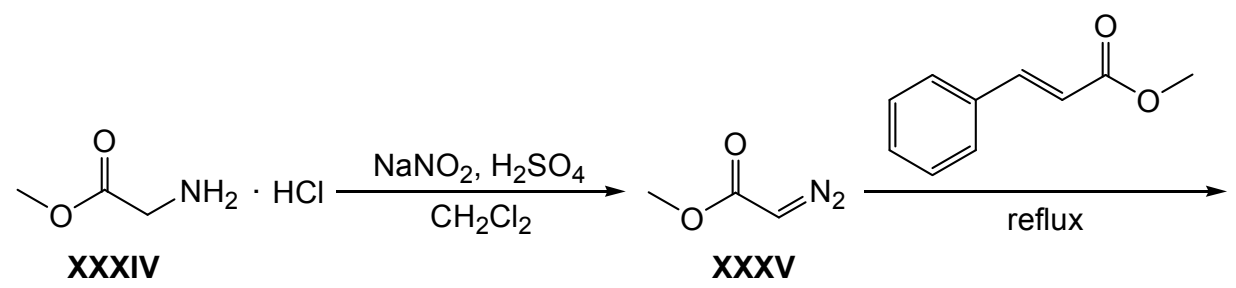<smiles>COC(=O)C1=NNC(C(=O)OC)C1c1ccccc1</smiles>

$\mathbf{X X X V I}$

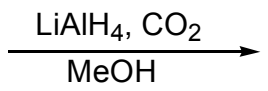

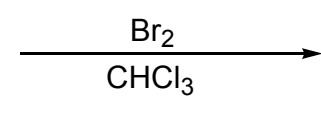<smiles>OCc1n[nH]c(CO)c1-c1ccccc1</smiles>

XXXVIII<smiles>COC(=O)c1n[nH]c(C(=O)OC)c1-c1ccccc1</smiles>

XXXVII

Scheme 21. Synthetic route for the preparation of 3,5-bis(hydroxymethyl)-4-phenyl-pyrazole.

XXXVIII could be further chlorinated using $\mathrm{SOCl}_{2}$ (as depicted in Scheme 10) and this derivative was then finally used for the coupling with amine XXXIII using the same conditions as described above (Scheme 22).<smiles>CCCc1[nH]c(CCl)c(-c2ccccc2)c1CCl</smiles>

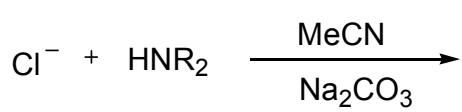<smiles>[R16]Cc1cc(CN[R16])[nH]n1</smiles>



$\left(\mathrm{HL}^{5}\right)$

Scheme 22. Preparation of the ligand $\mathbf{H L}^{5}$.

Thus, a new ligand $\mathbf{H L}^{\mathbf{5}}$, with a 4-phenyl substituted pyrazole as the core of the ligand, was prepared as the first representative of a new family of pyrazole-based compartmental ligands featuring an aryl-substituted pyrazole ring. 


\section{Synthesis and characterization of complexes}

\subsection{Complex synthesis: general procedure}

Pyrazole-bridging ligands $\mathbf{H L}^{\mathbf{1}} \mathbf{-} \mathbf{H L}^{\mathbf{5}}$, each consisting of two tripodal tetradentate $\left\{\mathrm{N}_{4}\right\}$ subunits bridged by a pyrazole motif, serve to form specific, targeted bimetallic copper complexes. The general route for preparing such complexes is depicted in Scheme 23. The nature of the side arms (aliphatic or aromatic) of the pyrazole-based ligands does not play any significant role when choosing the synthetic strategy. To obtain such dicopper species, the pyrazole ligand has to be dissolved in $\mathrm{MeOH}$ or $\mathrm{MeCN}$, or in a mixture of these solvents, followed by addition of two equivalents of base. The first equivalent serves to deprotonate the pyrazole NH-functionality, which generates a dinucleating pyrazolate fragment, while the second equivalent of base is used to deprotonate either a solvent molecule or to abstract a proton from an $\mathrm{H}_{2} \mathrm{O}$ molecule, originating from the hydrated copper(II) salts employed.
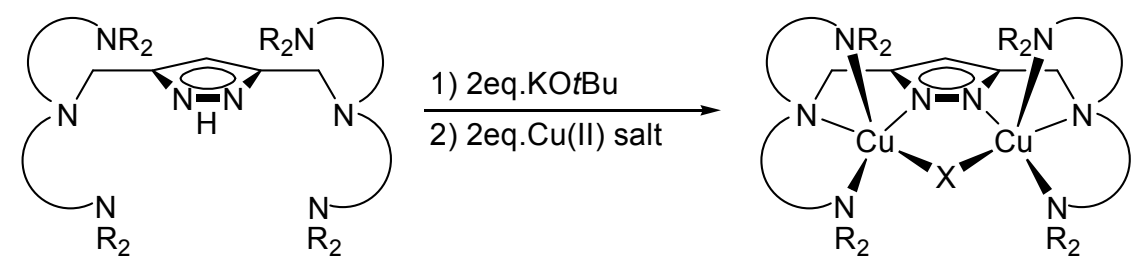

Scheme 23. Synthesis of the dicopper(II) complexes. The $\mathrm{X}$ ligand represents either a $\mathrm{MeO} \cdots \mathrm{HOMe}$, an $\mathrm{HO} \cdots \mathrm{HOH}$ or an $\mathrm{OH}$ bridging pattern.

Subsequent addition of two equivalents of copper(II) salt leads to the formation of the desired dinuclear $\mathrm{Cu}$-species, with each copper ion coordinated within one of the two tripodal $\left\{\mathrm{N}_{4}\right\}$ binding pockets, with either a $\mathrm{MeO} \cdots \mathrm{HOMe}$, an $\mathrm{HO} \cdots \mathrm{HOH}$ or $\mu-\mathrm{OH}$ motif, obtained after the second deprotonation step. From the wide range of available copper(II) salts, those containing large, weakly coordinated anions, such as $\mathrm{ClO}_{4}^{-}, \mathrm{NO}_{3}{ }^{-}$or $\mathrm{BF}_{4}^{-}$were selected to be studied primarily. In these complex-formation reactions, $\mathrm{KO} t \mathrm{Bu}$ is normally used as a base in order to enable easy removal of the byproduct formed during the reaction, e.g. $\mathrm{KClO}_{4}$, by precipitation. The isolated copper complex can be redissolved in the appropriate solvent, whereafter slow diffusion of $\mathrm{Et}_{2} \mathrm{O}$ into this solution generally yields single crystals suitable for X-ray analysis. 


\subsubsection{Purification of complexes}

Traces of the incompletely reacted amine XXXIII still present after the synthesis of ligands $\mathbf{H L}^{4}$ and $\mathbf{H L}^{5}$ proved to have a detrimental influence on the isolation of pure dicopper species, because of formation and co-crystallization of mononuclear copper complexes. Therefore, purification of each crude mixture of $\mathrm{Cu}$-complexes was performed under gravity flow using $\mathrm{Na}^{+}-$loaded SP Sephadex C25 (40-125 $\mu$ ) cation-exchange resin.

SP-Sephadex C25 consists of macroscopic beads that are synthetically derived from the polysaccharide dextran, with strongly acidic propylsulfate (sulfopropyl or SP) groups fixed on the surface (Scheme 24).

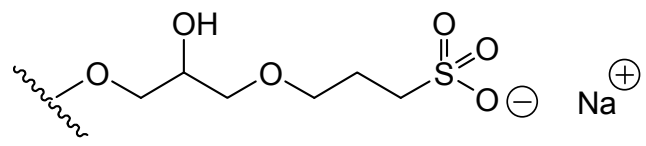

Scheme 24. Schematic representation of the "sulfopropyl" (SP) groups of the cationic exchang resin SP-Sephadex C25.

A solution of the particular copper complex subjected to this purification/separation procedure was prepared applying the same synthetic route described above with minor changes (only one equivalent of base was used). After addition of the copper salt, a further dilution of the reaction mixture with water to reach a ratio between organic solvent:water of 1:10 is required. The principle of ion-exchange column chromatography is based on interaction of the cationic part of the (in this case) Cu-complex present in the solution with the Sephadex column material; retention is dependent on the overall charge of the complex, with highly charged species (in the present study; multinuclear species) eluting slower then mononuclear analogues. Separation of the different complexes formed was carried out by washing the column with various concentrations of an aqueous solution of $\mathrm{NaNO}_{3}$.

The fraction of the singly charged mononuclear copper species derived from the incompletely reacted amine was collected after elution with $0.2 \mathrm{M} \mathrm{NaNO}_{3}$ solution. Subsequent elution with $0.4 \mathrm{M} \mathrm{NaNO}_{3}$ solution resulted in isolation of the main product of the complexation reaction, i.e. a multinuclear copper complex. Additional treatment of this main fraction 
(changing of $\mathrm{pH}$ or solvents with regard to complex solubility) led to the formation and isolation of the desired dicopper species.

\subsection{Copper(II) complexes of ligands $\mathrm{HL}^{1}$ and $\mathrm{HL}^{2}$}

In order to obtain a detailed understanding about the type of complexes formed both in solution and the solid state, the coordination behaviour of the two ligands $\mathbf{H L}^{\mathbf{1}}$ and $\mathbf{H L}^{\mathbf{2}}$, containing functionalized aliphatic side arms, towards copper is studied. The influence of the reaction conditions on the formation of different types of dicopper complexes is discussed.

\subsubsection{Structural characterization of copper complex with $\mathrm{HL}^{1}$}

Reaction of $\mathbf{H L}^{1}$ with two equivalents of $\mathrm{KO} t \mathrm{Bu}$ in $\mathrm{MeOH} / \mathrm{MeCN}$, followed by addition of two equivalents of $\mathrm{Cu}\left(\mathrm{BF}_{4}\right)_{2} \cdot 4 \mathrm{H}_{2} \mathrm{O}$ to the reaction mixture led to the formation of dinuclear copper complex 1 (Figure 16).

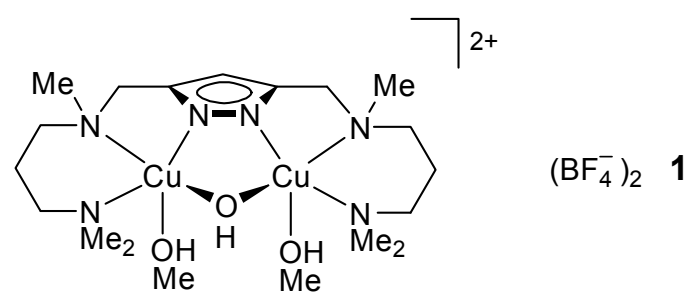

Figure 16. Structure of complex 1.

Single crystals of complex $\mathbf{1}$, obtained by diffusion of the $\mathrm{Et}_{2} \mathrm{O}$ into a methanol solution, were used for an X-ray crystallographic analysis. The molecular structure of the cation of $\mathbf{1}$ is shown in Figure 17.

As was previously described, pyrazole ligands such as $\mathbf{H L}^{\mathbf{1}}$, with (relatively) long and flexible side arms, favor a short intramolecular copper-copper distance. Furthermore, depending on the number of $\mathrm{N}$-donors, coordination of additional co-ligands (either solvent molecules or counter anions) can be observed. ${ }^{71}$ 


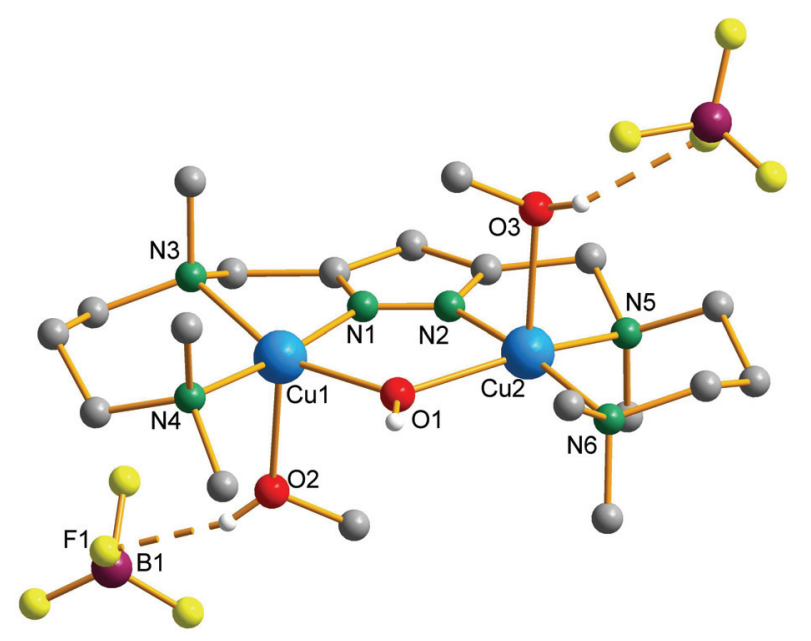

Figure 17. The molecular structure of complex 1.

Two copper(II) ions are nested within their respective coordination pockets and are spanned by the bridging pyrazolate moiety. Each copper atom is in a roughly square planar coordination environment $(\tau \mathrm{Cu} 1=0.23, \tau \mathrm{Cu} 2=0.24),{ }^{106}$ consisting of three $\mathrm{N}$-donors from the pyrazolate ligand and an $\mathrm{O}$ atom from the $\mu-\mathrm{OH}$-unit located within the bimetallic pocket. An additional axial position is occupied by a solvent molecule $(\mathrm{MeOH})$ at a greater distance (2.25 - $2.28 \AA$ ), typical for Jahn-Teller distortion (Table 1). The distance between the copper atom and the oxygen atom from the co-ligand in complex $\mathbf{1}$ is short in comparison to known complexes based on the same ligand with aliphatic compartments, where the $\mathrm{Cu}-\mathrm{O}$ separation is typically in the range from 2.32 to $2.36 \AA$. Fluorine atoms from two $\mathrm{BF}_{4}$ counter-anions and the additional axially coordinated $\mathrm{MeOH}$ ligands participate in hydrogen-bonding. The rather short $\mathrm{Cu} \cdots \mathrm{Cu}$ distance of $3.60 \AA$ is due to the long chelating side arms appended to the pyrazole, which provide formation of the small hydroxo-bridge, without any additionally included solvent molecule within the bimetallic pocket. The UV/vis spectroscopic measurements completely agreed with the persistence of the square planar coordination environment of the copper ions upon dissolution in MeCN, with only one observed $d-d$ transition band at $\lambda_{\max }=628 \mathrm{~nm}$. 
Table 1. Selected intramolecular distances $(\AA)$ and angles $\left(^{\circ}\right)$ for complex $\mathbf{1}$.

\begin{tabular}{|c|c|c|c|}
\hline \multicolumn{4}{|c|}{ Distances } \\
\hline $\mathrm{Cu}(1)-\mathrm{N}(1)$ & $1.849(6)$ & $\mathrm{Cu}(2)-\mathrm{N}(6)$ & $2.030(7)$ \\
\hline $\mathrm{Cu}(1)-\mathrm{N}(4)$ & $1.996(5)$ & $\mathrm{Cu}(2)-\mathrm{N}(5)$ & $2.102(6)$ \\
\hline $\mathrm{Cu}(1)-\mathrm{N}(3)$ & $2.113(7)$ & $\mathrm{Cu}(2)-\mathrm{O}(1)$ & $2.189(5)$ \\
\hline $\mathrm{Cu}(1)-\mathrm{O}(1)$ & $2.115(7)$ & $\mathrm{Cu}(2)-\mathrm{O}(3)$ & $2.289(6)$ \\
\hline $\mathrm{Cu}(1)-\mathrm{O}(2)$ & $2.250(6)$ & $\mathrm{Cu}(1) \cdots \mathrm{Cu}(2)$ & $3.596(1)$ \\
\hline $\mathrm{Cu}(2)-\mathrm{N}(2)$ & $1.871(8)$ & & \\
\hline \multicolumn{4}{|c|}{ Angles } \\
\hline $\mathrm{N}(1)-\mathrm{Cu}(1)-\mathrm{N}(4)$ & $173.5(3)$ & $\mathrm{N}(2)-\mathrm{Cu}(2)-\mathrm{N}(6)$ & $172.5(3)$ \\
\hline $\mathrm{N}(1)-\mathrm{Cu}(1)-\mathrm{N}(3)$ & $79.0(3)$ & $\mathrm{N}(2)-\mathrm{Cu}(2)-\mathrm{N}(5)$ & $81.6(3)$ \\
\hline $\mathrm{N}(4)-\mathrm{Cu}(1)-\mathrm{N}(3)$ & $98.8(3)$ & $\mathrm{N}(6)-\mathrm{Cu}(2)-\mathrm{N}(5)$ & $98.3(3)$ \\
\hline $\mathrm{N}(1)-\mathrm{Cu}(1)-\mathrm{O}(1)$ & $86.0(3)$ & $\mathrm{N}(2)-\mathrm{Cu}(2)-\mathrm{O}(1)$ & $82.8(3)$ \\
\hline $\mathrm{N}(4)-\mathrm{Cu}(1)-\mathrm{O}(1)$ & $94.6(3)$ & $\mathrm{N}(6)-\mathrm{Cu}(2)-\mathrm{O}(1)$ & $95.2(3)$ \\
\hline $\mathrm{N}(3)-\mathrm{Cu}(1)-\mathrm{O}(1)$ & $159.6(2)$ & $\mathrm{N}(5)-\mathrm{Cu}(2)-\mathrm{O}(1)$ & $158.1(2)$ \\
\hline $\mathrm{O}(1)-\mathrm{Cu}(1)-\mathrm{O}(2)$ & $95.2(2)$ & $\mathrm{O}(1)-\mathrm{Cu}(2)-\mathrm{O}(3)$ & $97.1(2)$ \\
\hline $\mathrm{N}(1)-\mathrm{Cu}(1)-\mathrm{O}(2)$ & $92.8(3)$ & $\mathrm{N}(2)-\mathrm{Cu}(2)-\mathrm{O}(3)$ & $90.0(3)$ \\
\hline $\mathrm{N}(3)-\mathrm{Cu}(1)-\mathrm{O}(2)$ & $99.2(2)$ & $\mathrm{N}(5)-\mathrm{Cu}(2)-\mathrm{O}(3)$ & $98.1(2)$ \\
\hline $\mathrm{N}(4)-\mathrm{Cu}(1)-\mathrm{O}(2)$ & $93.6(2)$ & $\mathrm{N}(6)-\mathrm{Cu}(2)-\mathrm{O}(3)$ & $97.4(3)$ \\
\hline
\end{tabular}

\subsubsection{Previously studied catalytic activity of dicopper complexes}

Dicopper(II) complexes based on a set of ligands with aliphatic side arms were previously tested as catalysts for the oxidative polymerization and coupling of 2,6-dimethylphenol (DMP) (Table 2). Poly(2,6-dimethylphenylene ether) (PPE, an important ingredient of highperformance engineering plastics) was the main product obtained under all chosen reaction conditions (Scheme 25). ${ }^{107}$ 


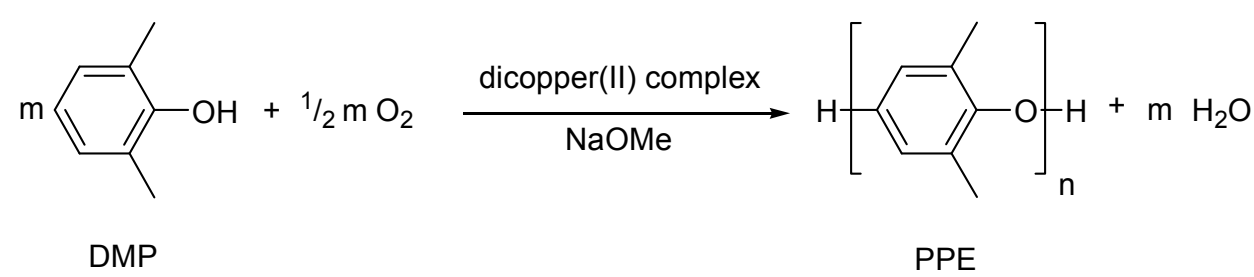

Scheme 25. Oxidative polymerization of DMP catalyzed by pyrazolate-based dicopper(II) complexes.

Table 2. Chelating side arms appended to the pyrazole unit and $\mathrm{Cu} \cdots \mathrm{Cu}$ separation $(\AA)$ found in the dicopper(II) complexes used for polymerization of DMP.

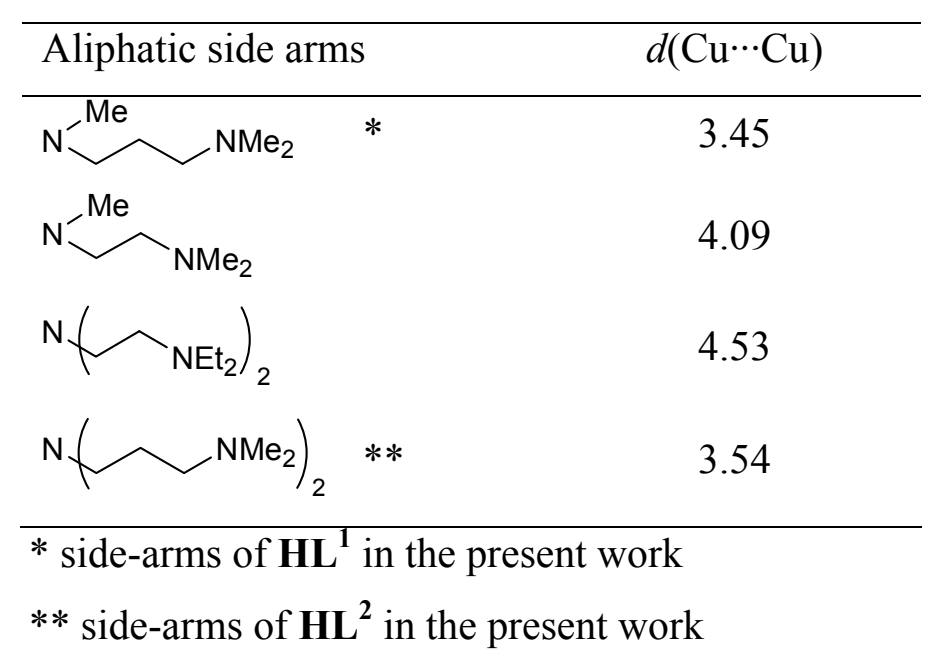

Complex 2 (based on $\left.\mathbf{H L}^{2}\right)^{107,108}$ was found to be the most active catalyst for the oxidative polymerization (C-O coupling), but activity only occurred when two additional equivalents of base $(\mathrm{NaOMe})$ were added to the reaction mixture in $\mathrm{MeCN}$. It was assumed that supplementary base was necessary for deprotonation of two dangling, protonated amine side arms, followed by the formation of complex $\mathbf{2 a}$. This latter complex, derived from $\mathbf{2}$ by deprotonation and coordination of two additional amine groups, was suggested to be the active species in the polymerization of DMP (Scheme 26). 




2

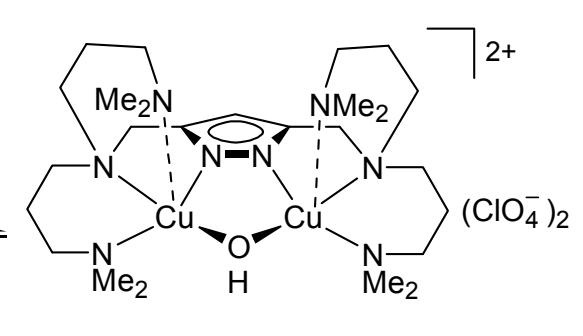

$2 a$

Scheme 26. Schematic representation of the assumed interconversion between $\mathbf{2}$ and $\mathbf{2 a}$.

Dicopper complex 2 resembles the structure found for complex 1, with both copper atoms in identical square pyramidal coordination environments. Two $N$-dimethyl-aminopropyl groups of $\mathbf{H L}^{2}$ are protonated and therefore remain uncoordinated; as such they can be considered analogous to the methyl groups in $\mathbf{H L}^{\mathbf{1}}$. In this sense, the fifth, axial position of the copper atoms in $\mathbf{1}$ is occupied by coordinating solvent molecules, while in $\mathbf{2}$ coordination of the counter anions as co-ligands was observed in the same fashion. ${ }^{71}$

\subsubsection{Equilibria in solution and structural characterization of complexes with $\mathrm{HL}^{2}$ : unusual copper-dioxygen mediated amine to $N$-oxide transformation of $\mathrm{HL}^{2}$}

In order to gain insight in the role of the supplementary base used during the activation of complex $\mathbf{2}$ and prior to any polymerization of DMP, the possible existence of an equilibrium between 2 and 2a was studied. Dicopper complex $\mathbf{2}$ was dissolved in a mixture of $\mathrm{MeOH} / \mathrm{MeCN}\left(0.0049 \mathrm{~mol} \mathrm{~L}^{-1}\right)$ and addition of the $\mathrm{KO} t \mathrm{Bu}$ solution $\left(0.0098 \mathrm{~mol} \mathrm{~L}^{-1}\right)$ was performed in a stepwise manner (Figure 18). 


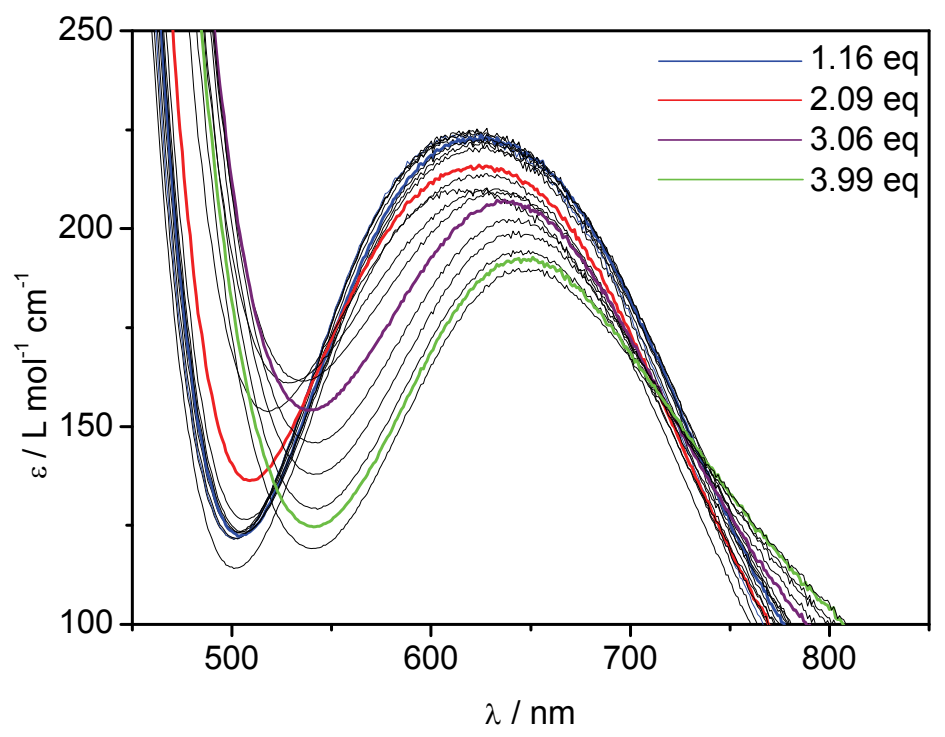

Figure 18. UV/vis titration of a solution of 2 in $\mathrm{MeOH} / \mathrm{MeCN}$ with $\mathrm{KO} t \mathrm{Bu}$.

The initial dicopper species 2 showed a characteristic absorption ( $d-d$ band) at $\lambda_{\max }$ of $618 \mathrm{~nm}$ and during titration with base, this maximum shifted gradually to lower energy, with the final value of $649 \mathrm{~nm}$ reached after four equivalents. However, no spectroscopic changes were observed when up to two equivalents of base were added and only the addition of a further two equivalents of $\mathrm{KO} t \mathrm{Bu}$ induced a shift towards weak ligand field (Figure 19).

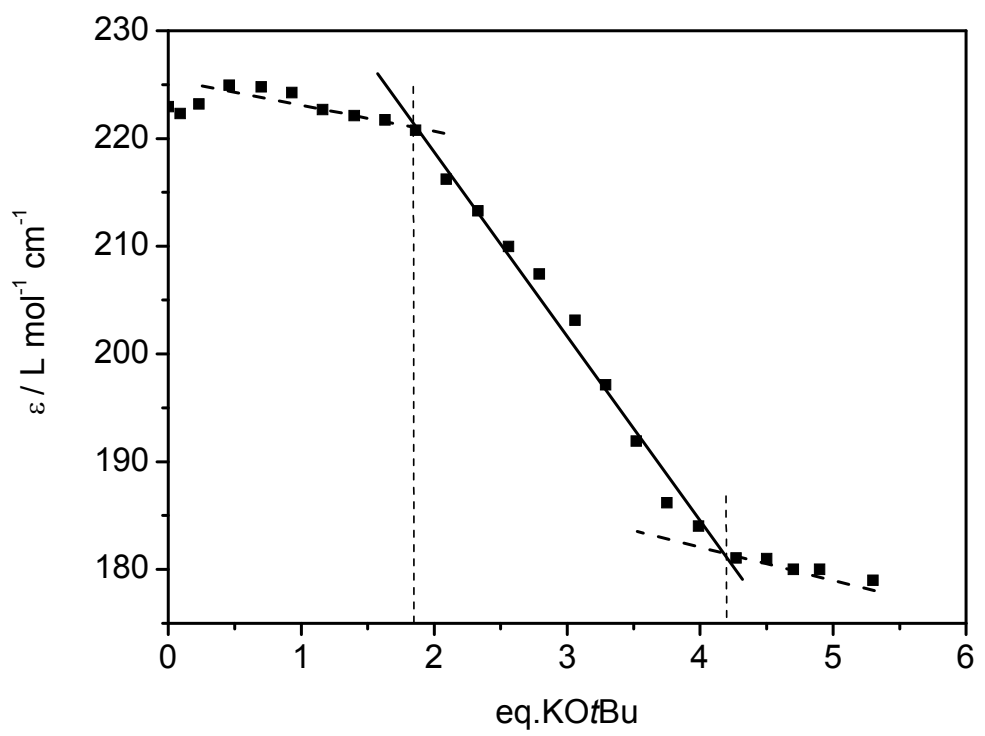

Figure 19. Changes in extinction coefficient depending on the amount of base added. 
The necessity to add four equivalents of base to drive the (equilibrium) reaction to completion showed that the assumed single-step equilibrium between $\mathbf{2}$ and $\mathbf{2 a}$ (see above) is most likely a more complicated multistep-process. One can propose that the protonated amine side arms in 2 are each losing one proton upon addition of the first two equivalents of base and thereafter loosely coordinate to the dicopper core. Coordination of the long, initially protonated side arms in the axial positions would not cause significant changes to the square pyramidal geometry of the copper ions, which is in a good agreement with the observed small shift of the band at $618 \mathrm{~nm}$ in the UV/vis spectrum. Further addition of the second two equivalents of base could then lead to deprotonation of two solvent molecules $(\mathrm{MeOH})$, which would subsequently become coordinated to the copper ions via exchange with the initial coligand $\left(\mathrm{ClO}_{4}^{-}\right)$. This exchange induces structural/electronic changes in the complex, as observed in the UV/vis spectrum, where finally formed species showed an absorption maximum at a $\lambda_{\max }$ of $649 \mathrm{~nm}$.

Crystallization of the deprotonated species, anticipated to have the initially proposed structure as in $\mathbf{2 a}$ was performed from the reaction mixture of $\mathbf{H L}^{2}$ with two equivalents of $\mathrm{KO} t \mathrm{Bu}$ and two equivalents of $\mathrm{Cu}\left(\mathrm{ClO}_{4}\right)_{2} \cdot 6 \mathrm{H}_{2} \mathrm{O}$ in $\mathrm{MeOH}$ or EtOH. However, in sharp contrast with the expected results, the X-ray crystallographic analysis on two different kinds of crystals yielded the molecular structures $\mathbf{3}$ and $\mathbf{3 a}$ (Figure 20). A similar structure $\mathbf{3 b}$ was obtained when using $\mathrm{Cu}\left(\mathrm{BF}_{4}\right)_{2} \cdot 4 \mathrm{H}_{2} \mathrm{O}$ as the copper salt (see below).

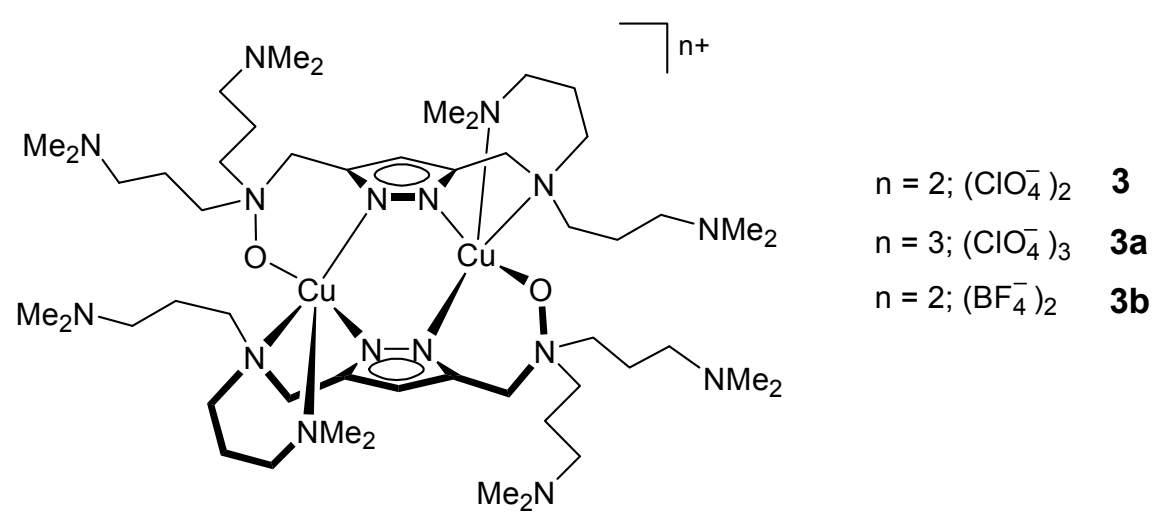

Figure 20. General structure of the $N$-oxide type of complexes $\mathbf{3}, \mathbf{3 a}$ and $\mathbf{3 b}$. $\mathbf{3 a}$ contains a proton not localized in the X-ray structure.

The common feature shared between the different molecular structures found for $\mathbf{3 - 3 b}$ is a drastic modification of the ligand, consisting of oxidation of one tertiary, bridgehead amine of 
the side arms, yielding the $N$-oxide functionality. This oxidation results in the formation of an unexpected and unusual bonding motif, wherein the two copper atoms are coordinated to the oxygen atom of the oxidized amine ( $N$-oxide) fragment.

The only difference observed in the molecular structures between $\mathbf{3}$ and $\mathbf{3 a}$ (the molecular structure of the latter is depicted in Figure 21) is the protonated state of one of the uncoordinated $\mathrm{N}$-dimethyl-aminopropyl groups in $\mathbf{3 a}$, which increases the amount of perchlorate counter anions and induces the asymmetric structure (i.e. the copper atoms are non-equivalent). The protonation also results in a distortion of the coordination geometry of the copper ions. Thus, whereas the crystallographically equivalent copper ions in $\mathbf{3}$ have almost perfect tetragonal geometry $(\tau=0.03)$, in complex 3a the $\tau$-value increases to around 0.20 for the second copper atom (Table 4).

In both complexes the copper atoms are ligated by three nitrogens from one of the (partly non-oxidized) coordinated ligands and the two remaining coordination positions are taken up by a nitrogen from the pyrazole unit and the oxygen atom of the $\mathrm{N}-\mathrm{O}$ fragment stemming from the second coordinated ligand.

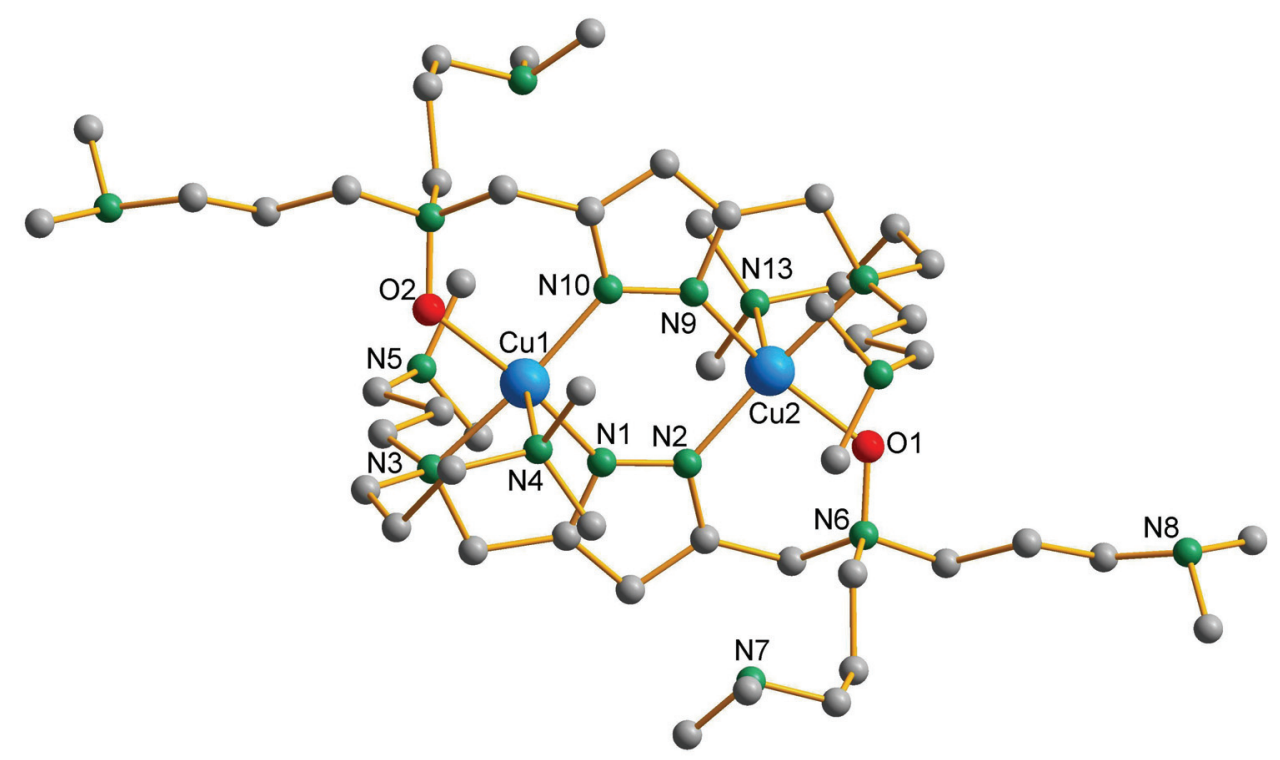

Figure 21. Molecular structure of the cation of $\mathbf{3 a}$. 


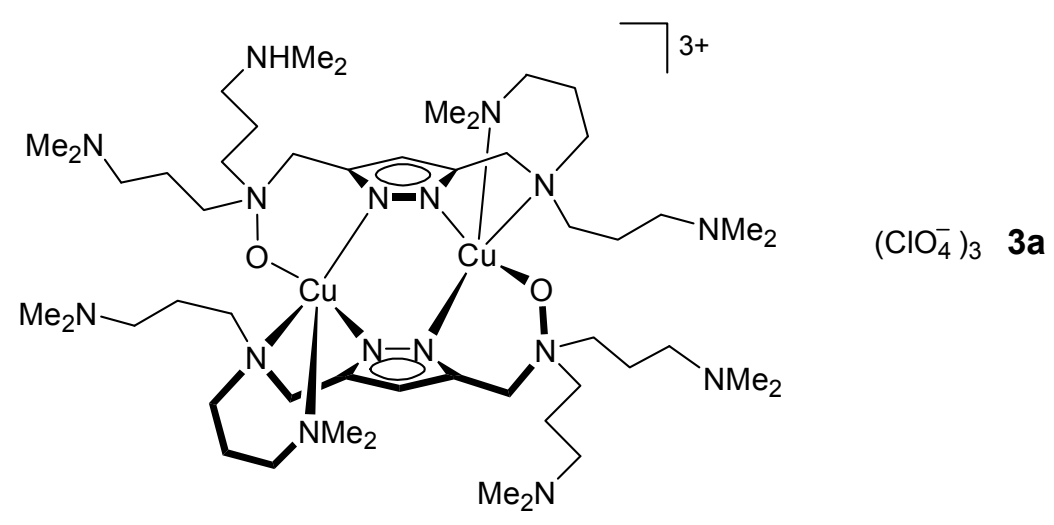

Figure 22. Structure of the $N$-oxide type of complex 3a which contains a proton not localized in the X-ray structure.

Table 3. Selected atom distances $(\AA)$ and angles $\left(^{\circ}\right)$ for complex $\mathbf{3 a}$.

\begin{tabular}{|c|c|c|c|}
\hline \multicolumn{4}{|c|}{ Distances } \\
\hline $\mathrm{Cu}(1)-\mathrm{O}(2)$ & $1.937(7)$ & $\mathrm{Cu}(2)-\mathrm{O}(1)$ & $1.967(6)$ \\
\hline $\mathrm{Cu}(1)-\mathrm{N}(1)$ & $1.962(9)$ & $\mathrm{Cu}(2)-\mathrm{N}(11)$ & $2.125(11)$ \\
\hline $\mathrm{Cu}(1)-\mathrm{N}(10)$ & $1.978(11)$ & $\mathrm{Cu}(2)-\mathrm{N}(13)$ & $2.359(8)$ \\
\hline $\mathrm{Cu}(1)-\mathrm{N}(3)$ & $2.074(12)$ & $\mathrm{Cu}(1) \cdots \mathrm{Cu}(2)$ & $3.931(2)$ \\
\hline $\mathrm{Cu}(1)-\mathrm{N}(4)$ & $2.359(9)$ & $\mathrm{N}(6)-\mathrm{O}(1)$ & $1.386(12)$ \\
\hline $\mathrm{Cu}(2)-\mathrm{N}(9)$ & $1.946(9)$ & $\mathrm{N}(14)-\mathrm{O}(2)$ & $1.434(12)$ \\
\hline $\mathrm{Cu}(2)-\mathrm{N}(2)$ & $1.954(11)$ & & \\
\hline \multicolumn{4}{|c|}{ Angles } \\
\hline $\mathrm{O}(2)-\mathrm{Cu}(1)-\mathrm{N}(1)$ & $162.6(3)$ & $\mathrm{N}(9)-\mathrm{Cu}(2)-\mathrm{N}(2)$ & $97.8(4)$ \\
\hline $\mathrm{O}(2)-\mathrm{Cu}(1)-\mathrm{N}(10)$ & $91.9(3)$ & $\mathrm{N}(9)-\mathrm{Cu}(2)-\mathrm{O}(1)$ & $161.0(3)$ \\
\hline $\mathrm{N}(1)-\mathrm{Cu}(1)-\mathrm{N}(10)$ & $97.9(4)$ & $\mathrm{N}(2)-\mathrm{Cu}(2)-\mathrm{O}(1)$ & $92.4(3)$ \\
\hline $\mathrm{O}(2)-\mathrm{Cu}(1)-\mathrm{N}(3)$ & $85.0(3)$ & $\mathrm{N}(9)-\mathrm{Cu}(2)-\mathrm{N}(11)$ & $82.1(4)$ \\
\hline $\mathrm{N}(1)-\mathrm{Cu}(1)-\mathrm{N}(3)$ & $82.6(4)$ & $\mathrm{N}(2)-\mathrm{Cu}(2)-\mathrm{N}(11)$ & $171.2(4)$ \\
\hline $\mathrm{N}(10)-\mathrm{Cu}(1)-\mathrm{N}(3)$ & $168.9(4)$ & $\mathrm{O}(1)-\mathrm{Cu}(2)-\mathrm{N}(11)$ & $85.4(3)$ \\
\hline $\mathrm{O}(2)-\mathrm{Cu}(1)-\mathrm{N}(4)$ & $96.8(3)$ & $\mathrm{N}(9)-\mathrm{Cu}(2)-\mathrm{N}(13)$ & $94.9(3)$ \\
\hline $\mathrm{N}(1)-\mathrm{Cu}(1)-\mathrm{N}(4)$ & $96.1(3)$ & $\mathrm{N}(2)-\mathrm{Cu}(2)-\mathrm{N}(13)$ & $97.3(4)$ \\
\hline $\mathrm{N}(10)-\mathrm{Cu}(1)-\mathrm{N}(4)$ & $97.5(3)$ & $\mathrm{O}(1)-\mathrm{Cu}(2)-\mathrm{N}(13)$ & $99.7(3)$ \\
\hline $\mathrm{N}(3)-\mathrm{Cu}(1)-\mathrm{N}(4)$ & $93.4(4)$ & $\mathrm{N}(11)-\mathrm{Cu}(2)-\mathrm{N}(13)$ & $91.4(4)$ \\
\hline
\end{tabular}


Table 4. $\tau$-value and the $\mathrm{Cu} \cdots \mathrm{Cu}$ distance $(\AA)$ for complexes $\mathbf{3}, \mathbf{3 a}$ and $\mathbf{3 b}$.

\begin{tabular}{lcc}
\hline Complex & $\tau(\mathrm{Cu} 1 / \mathrm{Cu} 2)$ & $d(\mathrm{Cu} \cdots \mathrm{Cu})$ \\
\hline $\mathbf{3}$ & 0.03 & 3.92 \\
3a & $0.10 / 0.17$ & 3.93 \\
3b & 0.06 & 3.92 \\
\hline
\end{tabular}

The N-O bond distances in the $N$-oxide copper(II) complexes are in the range from 1.41 to $1.43 \AA$ what is close to identical to those reported in the literature by Comba and coworkers. ${ }^{109}$

It has to be mentioned that the $N$-oxide complex is only formed during the long crystallization process (of up to one month). No $N$-oxide containing species were observed by means of ESI mass spectrometry in the reaction mixture before crystallization, while after crystallization the $N$-oxide species was clearly detected. Thus, for complex $\mathbf{3 a}$, where one of the side arms is protonated, signals at 1189, 1289 and $1389 \mathrm{~m} / \mathrm{z}$ were observed, which confirm the presence of the species $\left[\mathrm{Cu}_{2} \mathrm{~L}_{2}^{2} \mathrm{O}_{2}\left(\mathrm{ClO}_{4}\right)\right]^{+},\left[\mathrm{Cu}_{2} \mathrm{~L}_{2}{ }_{2} \mathrm{O}_{2}\left(\mathrm{ClO}_{4}\right)_{2}\right]^{+}$and $\left[\mathrm{Cu}_{2} \mathrm{~L}_{2}{ }_{2} \mathrm{O}_{2}\left(\mathrm{ClO}_{4}\right)_{3}\right]^{+}$, respectively, in $\mathrm{MeOH}$ solution.

Although very rarely observed, it was shown by Karlin and co-workers that such copperdioxygen mediated amine to $N$-oxide transformation can be performed by a dicopper(I) complex based on a ligand with a built-in $N$-benzyl moiety (Figure 23). ${ }^{110}$
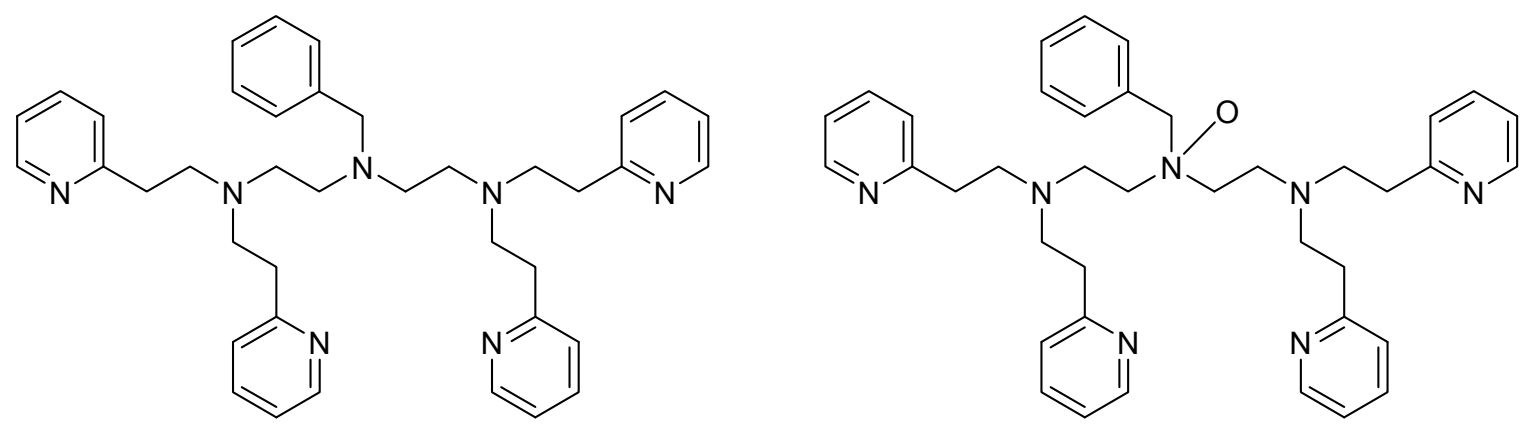

Figure 23. Ligand with a built-in $\mathrm{N}$-benzylic active moiety used by Karlin and isolated $\mathrm{N}$ oxide fragment, after decomposition of $\mathrm{Cu}_{2}{ }^{\mathrm{II}}-\mathrm{O}_{2}$ species.

After reaction of the dinuclear copper(I) species with $\mathrm{O}_{2}$ at $0{ }^{\circ} \mathrm{C}$ to yield a dicopper(II)-peroxo species, oxidation of the benzylic nitrogen and formation of the corresponding $\mathrm{N}$-oxide 
fragment had occurred, as a result of the decomposition of the peroxo species. Moreover, a labeling experiment using ${ }^{18} \mathrm{O}_{2}$ showed that the oxygen atom was derived from molecular dioxygen, which proved that the oxygen atom-transfer reaction was initiated by formation of the $\mathrm{Cu}_{2}{ }^{\mathrm{II}}-\mathrm{O}_{2}$ active species.

The proposed oxidation pathway by Karlin, however, can not be applied to the present amine to $N$-oxide transformation reaction since the copper ions in the initially prepared dicopper complex are already in the oxidation state +2 .

In order to determine the origin of the oxygen responsible for the oxidation of the tertiary amine, a copper salt with a non-oxygen containing counter-anion, such as $\mathrm{Cu}\left(\mathrm{BF}_{4}\right)_{2} \cdot 4 \mathrm{H}_{2} \mathrm{O}$, was used for the complex synthesis instead of the previously applied perchlorate salt. However, after approximately the same crystallization time, single crystals were again obtained and when the molecular structure was elucidated by X-ray crystallography, formation of dinuclear copper $N$-oxide complex $\mathbf{3 b}$ was unequivocally proven. $\mathbf{3 b}$ represents a close analogue of complex $\mathbf{3}$, where two copper atoms are crystallographically equivalent, with almost perfect tetragonal geometry, and a $d(\mathrm{Cu} \cdots \mathrm{Cu})$ equal to $3.92 \AA$.

Unfortunately, complete exclusion of all potential sources of oxygen was not possible with the experimental methods described here, since in the first place crystallization was only induced when performed in the presence of ether, whereas within the second synthetic route, the applied copper tetrafluoroborate salt was hydrated.

The possible influence of aerial dioxygen was firstly elucidated by bubbling pure oxygen gas through a solution of $\mathbf{H L}^{2}$ with two equivalents of $\mathrm{KO} t \mathrm{Bu}$ and two equivalents of $\mathrm{Cu}\left(\mathrm{ClO}_{4}\right)_{2} \cdot 6 \mathrm{H}_{2} \mathrm{O}$, and monitoring the $\mathrm{UV} /$ vis spectrum of the solution. No spectroscopic changes in the $d-d$ band region $(618 \mathrm{~nm})$ were observed during this procedure, indicating that formation of the $N$-oxide species is very slow, and only occurs during the crystallization period. To avoid the presence of any aerial dioxygen, reaction of $\mathbf{H L}^{2}$ with two equivalents of $\mathrm{KO} t \mathrm{Bu}$ and two equivalents $\mathrm{Cu}\left(\mathrm{ClO}_{4}\right)_{2} \cdot 6 \mathrm{H}_{2} \mathrm{O}$ was performed under anaerobic conditions in a glove box. The crystalline compound formed under these conditions was analyzed by means of ESI-MS spectrometry. The only signal found in the ESI spectrum at $591 \mathrm{~m} / \mathrm{z}$ corresponds to a $\left[\mathrm{Cu}_{2} \mathrm{~L}^{2}\right]^{+}$species, which is typically present in the reaction mixture before crystallization. Unfortunately, no single crystals were obtained to structurally prove the role of aerial dioxygen. On the basis of these observations, it can be concluded that no formation of $\mathrm{N}$ - 
oxide species takes place under anaerobic conditions, which implies that dioxygen is most likely responsible for the slow copper-dioxygen mediated amine to $N$-oxide transformation.

\subsection{Characterization of a dicopper complex with the new ligand $\mathrm{HL}^{3}$}

The coordination chemistry of a new pyrazole-based ligand with bis[(6-methyl-2pyridyl)methyl]amine side arms towards copper(II) was studied. Reaction of $\mathbf{H L}^{3}$ with two equivalents of $\mathrm{KO} t \mathrm{Bu}$ in $\mathrm{MeOH} / \mathrm{MeCN}$, followed by addition of two equivalents of $\mathrm{Cu}\left(\mathrm{ClO}_{4}\right)_{2} \cdot 6 \mathrm{H}_{2} \mathrm{O}$ to the reaction mixture led to the formation of dinuclear copper(II) complex $\left[\mathrm{Cu}_{2} \mathrm{~L}^{3}(\mathrm{OH})\right]\left(\mathrm{ClO}_{4}\right)_{2} 4$ (Figure 24).

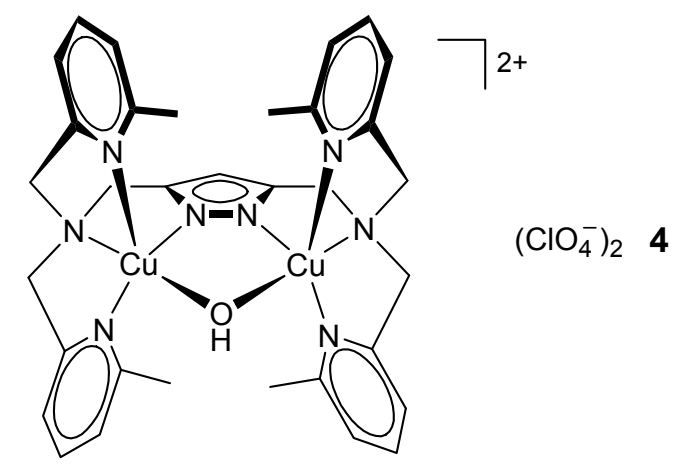

Figure 24. Schematic representation of complex 4.

The single crystals isolated after diffusion of $\mathrm{Et}_{2} \mathrm{O}$ into $\mathrm{CH}_{2} \mathrm{Cl}_{2} / \mathrm{MeCN}$ proved of insufficient quality for a high-resolution X-ray crystallographic analysis and therefore a complete dataset could not be obtained. However, the measurements did allow for a rough estimation of intramolecular distances between atoms with high electron density (like $\mathrm{Cu}, \mathrm{N}$ and $\mathrm{O}$ ), so that key information can still be discussed for this structure. The corresponding approximation of the molecular structure of $\mathbf{4}$ is shown in Figure 25. 


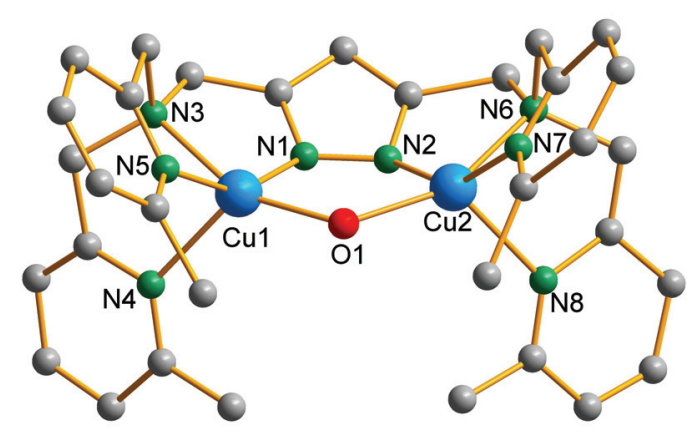

Figure 25. Molecular structure of the cation of 4 .

Two copper(II) ions are nested within their respective coordination pockets and are spanned by the bridging pyrazolate moiety. Both metal ions are five-coordinate with a slightly distorted trigonal bipyramidal geometry $(\tau=0.86)$, in accordance with the solution $U V / v i s$ data, where a $d-d$ transition band appeared at $\lambda_{\max }=816 \mathrm{~nm}$. The coordination sphere of both copper atoms consists of four N-donors from the pyrazolate ligand (one of the central pyrazole core and three from the side arm fragments) and a fifth bridgehead $\mathrm{O}$ atom, presumably originating from a $\mu-\mathrm{OH}$ ligand, located within the bimetallic pocket. The approximate distance between the two copper atoms is $c a 3.50 \AA$. This motif does not closely resemble the structural situation observed for some related $\mathrm{Ni}^{2+}$ and $\mathrm{Zn}^{2+}$ complexes of a pyrazole-derived ligand with bis(pyridyl methyl)amine side arms - without the methyl group in the 6-position - where the intramolecular metal-metal separation is larger than found for the two $\mathrm{Cu}$ ions in $\mathbf{4}$. Thus, in these dinuclear nickel(II) and dizinc(II) complexes, because of the short methylene-spacers in the side arms of the ligand, the metal ions are bridged by an acetate ligand, resulting in metal-metal separations of $c a 4.20 \AA$ and $3.97 \AA$, respectively. ${ }^{111,12}$ At the same time, a dicopper(II) complex derived from the ligand with short bis(pyridyl methyl)amine side arms showed a long intramolecular metal-metal separation of $4.30 \AA$ where within the resulting bimetallic pocket, the $\mathrm{F}$ atom and a methanol molecule form a strong hydrogen bond. ${ }^{113}$ The only reasonable signal, in terms of matching pattern, found in the ESI spectrum (MeCN solution), observed at $829 \mathrm{~m} / \mathrm{z}$, presumably corresponds to $\left[\mathrm{Cu}_{2} \mathrm{~L}^{3}(\mathrm{OH})(\mathrm{MeCN}) \mathrm{ClO}_{4}\right]^{+}$. 


\subsection{Copper complexes of the new bioinspired ligand $\mathrm{HL}^{4}$}

With the aim of further advancing the emulation of biological donor environments in pyrazole-based ligands, the new ligand $\mathbf{H L}^{4}$ that has binding compartments composed of imidazolyl groups has been prepared. To gain knowledge about the type of complexes with $\mathbf{H L}^{4}$, its coordination behaviour towards copper is studied. The complexation equilibria and the stability of copper(II) complexes of $\mathbf{H L}^{4}$ in solution as well as crystallographic and magnetic studies of the different complexes in the solid state are discussed.

\subsubsection{Elucidation of species distribution at different $\mathrm{pH}$ values}

The first studies were focussed on the elucidation of species distribution. Potentiometric titrations were carried out in order to determine the $\mathrm{p} K_{\mathrm{a}}$ values of the new ligand and to probe its copper(II) coordination chemistry. These studies were performed by Eva Anna Enyedy and Dr. Etelka Farkas at the University of Debrecen, Hungary.

Ligand protonation constants: Titrations were performed starting at acidic $\mathrm{pH}$ using a potassium hydroxide titrant. From the titration curves the deprotonation steps can be derived (see experimental part Figure 94 and Table 5). The processes occurring in the measurable $\mathrm{pH}$ range between ca. $\mathrm{pH} 4$ and 9 belong to the dissociation of one proton per protonated imidazole moiety. The difference between the sequential dissociation constants is higher (ca. $0.8 \log$ units) than expected for statistical reason, which suggests some interaction (most probably through space) between the individual protonation sites. According to the experimental results, protonation of the pyrazole occurs only below $\mathrm{pH} 2$ and the stability constant was not determined for this process. 
Table 5. Overall protonation constants $(\log \beta)$ and sequential dissociation constants $\left(\mathrm{p} K_{\mathrm{a}}\right)$ of the ligand $\mathbf{H L}^{4}$ at $25^{\circ} \mathrm{C} ; I=0.2 \mathrm{M}(\mathrm{KCl})^{\text {a }}$, for the sake of simplification all species in solution will be further described using $\mathbf{H L}$ instead of $\mathbf{H L}^{4}$.

\begin{tabular}{ccccc}
\hline & {$\left[\mathrm{LH}_{5}\right]^{4+}$} & {$\left[\mathrm{LH}_{4}\right]^{3+}$} & {$\left[\mathrm{LH}_{3}\right]^{2+}$} & {$\left[\mathrm{LH}_{2}\right]^{+}$} \\
\hline $\log \beta$ & $26.06(1)$ & $20.78(1)$ & $14.71(2)$ & $7.77(1)$ \\
$\mathrm{p} K_{\mathrm{a}}$ & 5.28 & 6.07 & 6.94 & 7.77
\end{tabular}

${ }^{a}$ Standard deviations are given in parenthesis

Species distribution of copper complexes: Titrations of $\mathbf{H L}^{4}$ in the presence of various equivalents of $\mathrm{Cu}^{\text {II }}$ were analyzed in batch calculations in which all titration curves are fitted at the same time with one model (see experimental part Figure 95 and Table 6). Evaluation of the titration curves shows that the $\mathrm{Cu}^{\mathrm{II}}$-binding capabilities of $\mathbf{H L}^{4}$ are high. Although free $\mathrm{Cu}^{\mathrm{II}}$ ions and the mononuclear species $\left[\mathrm{CuLH}_{3}\right]^{4+}$ are observable up to $\sim \mathrm{pH} 4$, the dinuclear species $\left[\mathrm{Cu}_{2} \mathrm{LH}\right]^{4+}$ is already formed at around $\mathrm{pH} 2$ and becomes almost the sole species around $\mathrm{pH} 4$ (Figure 26). This dinuclear complex, which is present at rather acidic conditions, presumably has a protonated (i.e. non-bridging) pyrazole unit. $\left[\mathrm{Cu}_{2} \mathrm{~L}\right]^{3+}$ starts to form above $\mathrm{pH} 4$ and exists in solution up to $\mathrm{pH} 10$.



Figure 26. Concentration distribution of the species formed between $\mathbf{H L}^{4}$ and $\mathrm{Cu}^{\mathrm{II}}$ at $1: 2$ ligand to metal ratio. $\mathrm{C}_{\mathrm{HL}}{ }^{4}=1.5 \cdot 10^{-3} \mathrm{M}$. 
It is the dominant species at $\mathrm{pH} 7$ and most likely is a pyrazolato-bridged complex, where the heterocycle is deprotonated. A further deprotonation step leads to dinuclear $\left[\mathrm{Cu}_{2} \mathrm{LH}_{-1}\right]^{2+}$ which is the major species under more basic conditions. The calculated $\mathrm{p} K_{\mathrm{a}}$ of 7.94 for $\left[\mathrm{Cu}_{2} \mathrm{~L}\right]^{3+}$ represents the acidity of a metal-bound water to give $\left[\mathrm{Cu}_{2} \mathrm{LH}_{-1}\right]^{2+}$, i.e. the latter species is better described as $\left[\mathrm{Cu}_{2} \mathrm{~L}(\mathrm{OH})\right]^{2+}$. The $\mathrm{Zn}^{2+}$ complex of tris(2-(1imidazolyl)methyl)amine shows a $\mathrm{p} K_{\mathrm{a}}$ of 8.72 for a metal-bound water, ${ }^{114}$ and the value for the corresponding mononuclear $\mathrm{Cu}^{\mathrm{II}}$ system (Figure 27, with $\mathrm{X}=\mathrm{H}_{2} \mathrm{O}$ ) should be even higher. $^{115}$



Figure 27. Schematic representation of the mononuclear copper complex with tris(2-(1imidazolyl)methyl)amine and $\mathrm{X}=\mathrm{H}_{2} \mathrm{O}$.

Hence, there is a clear increase in water acidity due to the bimetallic arrangement in $\left[\mathrm{Cu}_{2} \mathrm{~L}\right]^{3+}$, and its relatively low $\mathrm{p} K_{\mathrm{a}}$ value suggests a bridging position of the water or incorporation of the resulting hydroxide in a strongly hydrogen-bonded $\mathrm{O}_{2} \mathrm{H}_{3}$ moiety within the bimetallic pocket. However, acidification of the $\mathrm{Cu}$-bound water is less pronounced than in related pyrazolate-bridged systems with aliphatic N-donor side arms attached to the heterocycle. ${ }^{16}$

Table 6. Overall stability constants $(\log \beta)$ and some dissociation constants $\left(\mathrm{pK}_{\mathrm{a}}\right)$ for the complexes formed with $\mathrm{Cu}^{\text {II }}$ at $25^{\circ} \mathrm{C} ; I=0.2 \mathrm{M}(\mathrm{KCl}){ }^{\mathrm{a}}$

\begin{tabular}{ccccc}
\hline & {$\left[\mathrm{CuLH}_{3}\right]^{4+}$} & {$\left[\mathrm{Cu}_{2} \mathrm{LH}\right]^{4+}$} & {$\left[\mathrm{Cu}_{2} \mathrm{~L}\right]^{3+}$} & {$\left[\mathrm{Cu}_{2} \mathrm{LH}_{-1}\right]^{2+}$} \\
\hline $\log \beta$ & $26.36(4)$ & $23.56(3)$ & $17.79(8)$ & $9.85(9)$ \\
$\mathrm{p} K_{\mathrm{a}}$ & & 5.77 & 7.94 & \\
\hline
\end{tabular}

\footnotetext{
${ }^{\mathrm{a}}$ Standard deviations are given in parenthesis
} 
UV/vis spectra were recorded for aqueous solutions containing $\mathbf{H L}^{4}$ and two equivalents of $\mathrm{Cu}\left(\mathrm{NO}_{3}\right)_{2}$ at $\mathrm{pH} 4.66,6.55$, and 9.40 in order to further characterize the species $\left[\mathrm{Cu}_{2} \mathrm{LH}\right]^{4+}$, $\left[\mathrm{Cu}_{2} \mathrm{~L}\right]^{3+}$, and $\left[\mathrm{Cu}_{2} \mathrm{LH}_{-1}\right]^{2+}$ present under those conditions (Figure 28). The spectral features of the $\left[\mathrm{Cu}_{2} \mathrm{LH}_{-1}\right]^{2+}$ species at $\mathrm{pH} 9.40$ indicate a trigonal bipyramidal coordination geometry for both copper ions $\left(\lambda_{\max }=972 \mathrm{~nm}\right)$, while the species $\left[\mathrm{Cu}_{2} \mathrm{LH}\right]^{4+}$ and $\left[\mathrm{Cu}_{2} \mathrm{~L}\right]^{3+}$, which are present at $\mathrm{pH} 4.66$ and 6.55, respectively, have absorption maxima typical for copper in a square pyramidal environment $\left(\lambda_{\max } \approx 670 \mathrm{~nm}\right.$; Table 7$) .{ }^{116}$

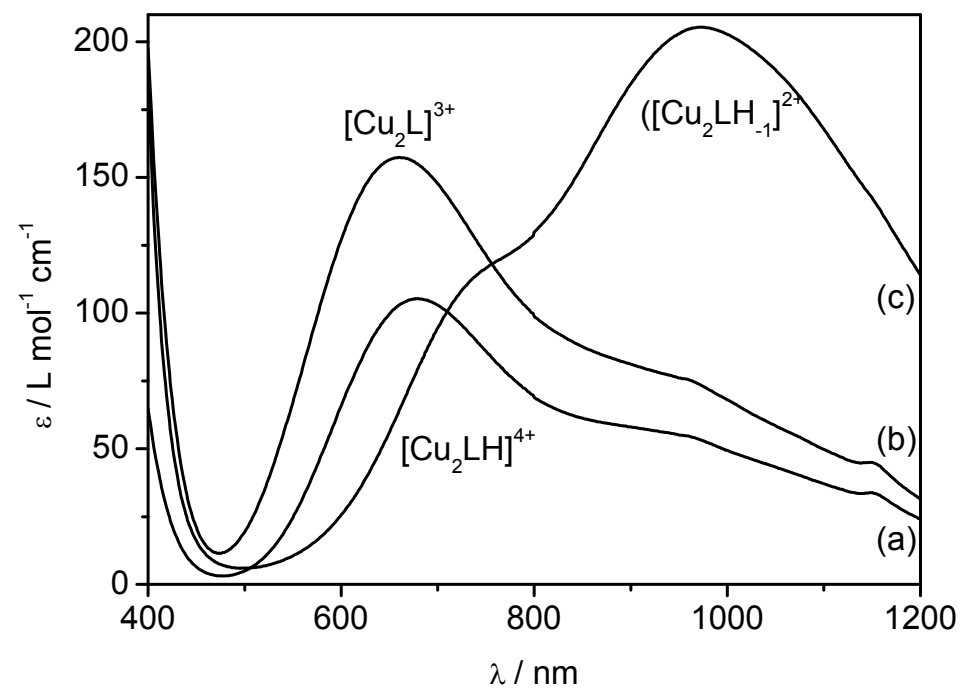

Figure 28. UV/vis spectra of $\mathbf{H L}^{4}$ with 2 equivalents of $\mathrm{Cu}\left(\mathrm{NO}_{3}\right)_{2}$ in $\mathrm{H}_{2} \mathrm{O}$ at $\mathrm{pH} 4.66$ (a), 6.55 (b) and 9.40 (c).

Table 7. UV/vis data of $\mathbf{H L}^{4}$ with 2 equivalents of $\mathrm{Cu}\left(\mathrm{NO}_{3}\right)_{2}$ in aqueous solution at different $\mathrm{pH}$ and of individual complexes 5a, 6, and 7 in $\mathrm{MeOH} / \mathrm{MeCN}(5: 2)$ and in the solid state (diffuse reflectance); $\lambda[\mathrm{nm}]\left(\varepsilon\left[\mathrm{L} \mathrm{mol}^{-1} \mathrm{~cm}^{-1}\right]\right)$.

\begin{tabular}{ccccc}
\hline species & $\mathrm{H}_{2} \mathrm{O}$ & complex & $\mathrm{MeOH} / \mathrm{MeCN}(5: 2)$ & $\begin{array}{c}\text { Diffuse } \\
\text { reflectance }\end{array}$ \\
\hline$\left[\mathrm{Cu}_{2} \mathrm{LH}_{-1}\right]^{2+}$ & $741(110), 972(204)$ & $\mathbf{5 a}$ & 730 sh $(135) 962(269)$ & 952 \\
{$\left[\mathrm{Cu}_{2} \mathrm{~L}\right]^{3+}$} & $663(157)$ & $\mathbf{6}$ & $689(281), 923(272)$ & 705,918 \\
{$\left[\mathrm{Cu}_{2} \mathrm{LH}\right]^{4+}$} & $676(105)$ & 7 & $677(96)$ & 689 \\
\hline
\end{tabular}




\subsubsection{Structural characterization of complexes}

Three distinctly different copper(II) complexes 5a - 7 of the pyrazole-based ligand $\mathbf{H L}^{4}$ could be isolated after work-up at appropriate $\mathrm{pH}$, reflecting the protonation state of the three different species $\left[\mathrm{Cu}_{2} \mathrm{LH}\right]^{4+},\left[\mathrm{Cu}_{2} \mathrm{~L}\right]^{3+}$, and $\left[\mathrm{Cu}_{2} \mathrm{LH}_{-1}\right]^{2+}$ (Figure 29). Single crystals of $\mathbf{5 a}, \mathbf{5 b}$, 6, and 7 were obtained and their molecular structures elucidated by X-ray crystallography.
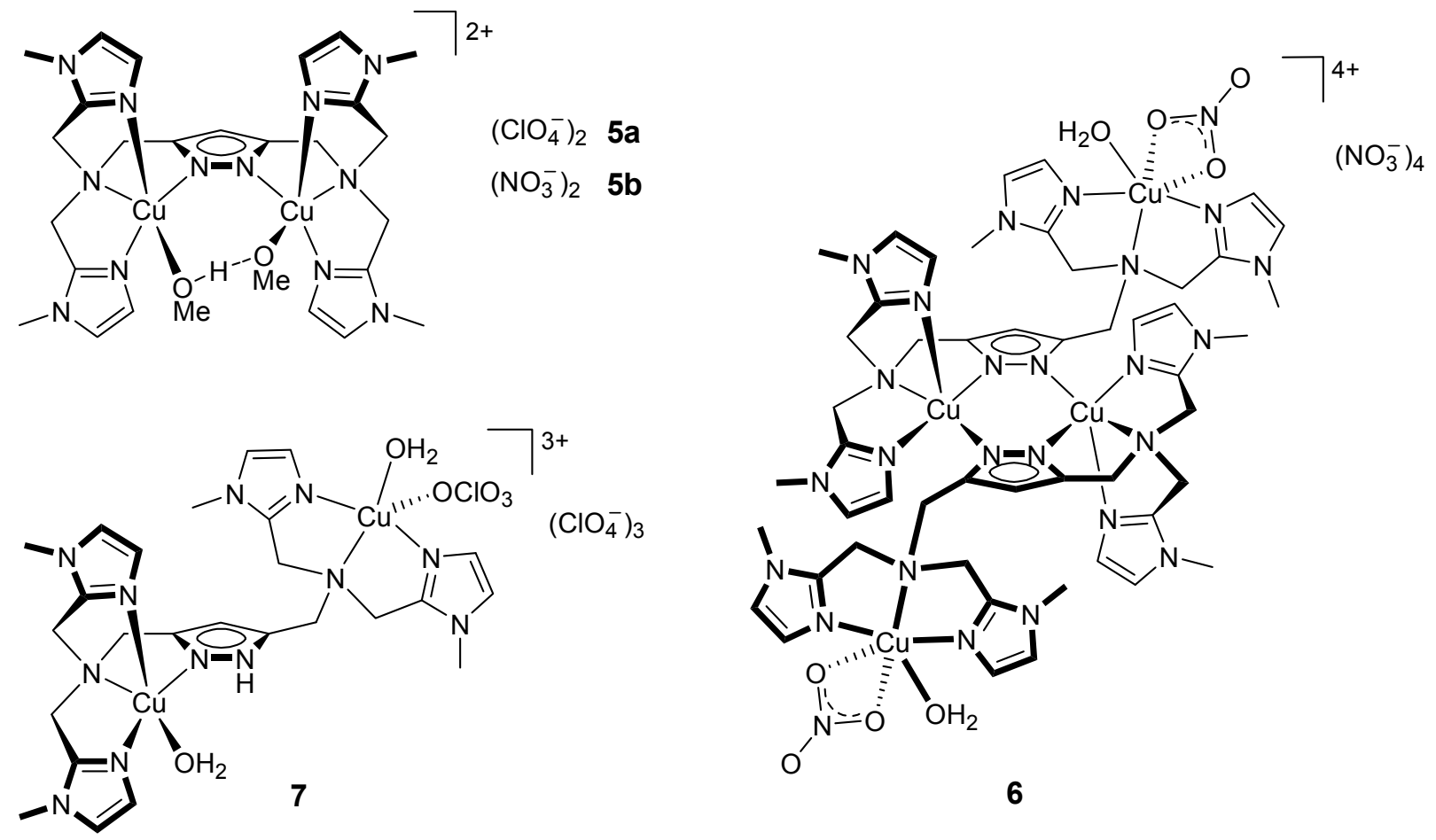

Figure 29. Schematic representation of copper complexes of ligand $\mathbf{H L}^{4}$ isolated under different $\mathrm{pH}$ conditions.

Addition of two equivalents of $\mathrm{Cu}\left(\mathrm{NO}_{3}\right)_{2}$ to a $\mathrm{MeOH} / \mathrm{H}_{2} \mathrm{O}$ solution containing $\mathbf{H L}^{4}$ and one equivalent of base $(\mathrm{KO} t \mathrm{Bu})$ produced a green-blue reaction mixture that was subjected to ionexchange chromatography using a SP-Sephadex C25 column. The first light-blue fraction was obtained after washing the column with a $0.2 \mathrm{M}$ solution of $\mathrm{NaNO}_{3}$. Interpretation of mass spectrometric signals $\left(330 \mathrm{~m} / z \quad\left[\mathrm{C}_{10} \mathrm{H}_{15} \mathrm{~N}_{5} \mathrm{CuNO}_{3}\right]^{+}, 268 \mathrm{~m} / z \quad\left[\mathrm{C}_{10} \mathrm{H}_{15} \mathrm{~N}_{5} \mathrm{Cu}\right]^{+}\right)$led to the conclusion that the compound obtained is a mononuclear copper complex, derived from the incompletely reacted $N, N$-bis[2-(1-methylimidazolyl)methyl]amine side-arm during ligand synthesis, with an isolated yield of $4.5 \%$. 
Washing of the column with a $0.4 \mathrm{M}$ solution of $\mathrm{NaNO}_{3}$ yielded the main blue aqueous fraction, which showed a characteristic maximum in the UV/vis spectrum at $656 \mathrm{~nm}$ (shifted in comparison to $663 \mathrm{~nm}$ due to the presence of $\mathrm{NaNO}_{3}$ in solution). By adjusting the $\mathrm{pH}$ of this solution to 9 and addition of $\mathrm{NaClO}_{4}$ a green powder precipitated that was crystallized from $\mathrm{MeCN} / \mathrm{MeOH} / \mathrm{Et}_{2} \mathrm{O}$ to yield complex 5a. The corresponding nitrate salt $\mathbf{5 b}$ could be prepared directly via the addition of two equivalents of base $(\mathrm{KO} t \mathrm{Bu})$ and two equivivalents of $\mathrm{Cu}\left(\mathrm{NO}_{3}\right)_{2}$ to a solution of $\mathbf{H L}^{4}$, followed by crystallization from $\mathrm{MeCN} / \mathrm{MeOH} / \mathrm{Et}_{2} \mathrm{O}$. Molecular structures of the cations of $\mathbf{5 a}$ and $\mathbf{5 b}$ are very similar, and the molecular structure of the former is shown in Figure 30.



Figure 30. Molecular structure of the cation of $\mathbf{5 a}$.

Table 8. Selected atom distances $(\AA)$ and angles $\left(^{\circ}\right)$ for complex $5 \mathbf{a}$.

\begin{tabular}{llll}
\hline \multicolumn{3}{c}{ Distances } \\
\hline Cu1-O1 & $1.915(2)$ & $\mathrm{Cu} 2-\mathrm{N} 2$ & $2.007(3)$ \\
$\mathrm{Cu} 1-\mathrm{N} 1$ & $2.030(3)$ & $\mathrm{Cu} 2-\mathrm{N} 8$ & $2.111(3)$ \\
$\mathrm{Cu} 1-\mathrm{N} 3$ & $2.129(3)$ & $\mathrm{Cu} 2-\mathrm{N} 9$ & $2.085(3)$ \\
$\mathrm{Cu} 1-\mathrm{N} 4$ & $2.080(3)$ & $\mathrm{Cu} 2-\mathrm{N} 11$ & $2.087(3)$ \\
$\mathrm{Cu} 1-\mathrm{N} 6$ & $2.013(3)$ & $\mathrm{Cu} 1 \cdots \mathrm{Cu} 2$ & $4.3405(6)$ \\
$\mathrm{Cu} 2-\mathrm{O} 2$ & $1.929(3)$ & $\mathrm{O} 1-\mathrm{O} 2$ & $2.407(4)$ \\
\hline & & Angles & $174.8(1)$ \\
\hline O1-Cu1-N1 & $96.4(1)$ & $\mathrm{O} 2-\mathrm{Cu} 2-\mathrm{N} 8$ & $104.2(1)$ \\
$\mathrm{O} 1-\mathrm{Cu} 1-\mathrm{N} 3$ & $177.3(1)$ & $\mathrm{O} 2-\mathrm{Cu} 2-\mathrm{N} 9$ & $96.9(1)$ \\
O1-Cu1-N4 & $101.5(1)$ & $\mathrm{O} 2-\mathrm{Cu} 2-\mathrm{N} 11$ & $80.3(1)$ \\
O1-Cu1-N6 & $96.4(1)$ & $\mathrm{N} 2-\mathrm{Cu} 2-\mathrm{N} 8$ &
\end{tabular}




$\begin{array}{llll}\text { N1-Cu1-N3 } & 80.8(1) & \text { N2-Cu2-N9 } & 118.5(1) \\ \text { N1-Cu1-N4 } & 109.1(1) & \text { N2-Cu2-N11 } & 121.4(1) \\ \text { N1-Cu1-N6 } & 124.2(1) & \text { N8-Cu2-N9 } & 80.1(1) \\ \text { N3-Cu1-N4 } & 81.0(1) & \text { N8-Cu2-N11 } & 78.8(1) \\ \text { N3-Cu1-N6 } & 81.4(1) & \text { N9-Cu2-N11 } & 110.7(1) \\ \text { N4-Cu1-N6 } & 119.47(1) & \text { O1-H1-O2 } & 173(5) \\ \text { O2-Cu2-N2 } & 99.8(1) & & \end{array}$

The two copper ions in 5a reside within the adjacent $\left\{\mathrm{N}_{4}\right\}$ ligand compartments of $\mathbf{L}^{4}$ and are bridged by the pyrazolate ring, as anticipated. Both metal ions are five-coordinate with a slightly distorted trigonal bipyramidal geometry $(\tau=0.88)$, in accordance with the solution $\mathrm{UV} / \mathrm{vis}$ data for $\left[\mathrm{Cu}_{2} \mathrm{LH}_{-1}\right]^{2+}$. The pyrazolate-N and the two imidazole-N atoms constitute the equatorial planes, while axial positions are occupied by the tertiary $\mathrm{N}$ atoms of the ligand backbone and by the $\mathrm{O}$ atoms of a $\mathrm{MeO} \cdots \mathrm{HOMe}$ moiety located within the bimetallic pocket. This motif closely resembles the situation observed for some $\mathrm{Ni}^{2+}, \mathrm{Cu}^{2+}$ and $\mathrm{Zn}^{2+}$ complexes of related pyrazole-derived ligands with pyridyl or aliphatic N-donor chelate substituents, where it was shown that the accessible range of metal-metal separations is determined by the length of the side arms appended to the pyrazole. ${ }^{15,16,81 b, 117}$ The rather short side arms of $\mathbf{H L}^{4}$ give five-membered chelate rings and thus enforce rather large $\mathrm{Cu} \cdots \mathrm{Cu}$ distances that prevent a small hydroxide or methoxide ligand from spanning the two metal ions. Incorporation of an additional $\mathrm{MeOH}$ solvent molecule then furnishes the $\mathrm{MeO} \cdots \mathrm{HOMe}$ bridging unit observed for $5 \mathbf{a}(d(\mathrm{Cu} \cdots \mathrm{Cu})=4.34 \AA)$. Such units have previously been shown to undergo rapid ligand exchange with e.g. water. ${ }^{117 \mathrm{~d}}$ Therefore the species $\left[\mathrm{Cu}_{2} \mathrm{LH}_{-1}\right]^{2+}$ that is present in aqueous solution at high $\mathrm{pH}$ most certainly contains an analogous $\mathrm{HO} \cdots \mathrm{HOH}$ bridge and should thus best be formulated as $\left[\mathrm{Cu}_{2} \mathrm{~L}\left(\mathrm{O}_{2} \mathrm{H}_{3}\right)\right]^{2+}$.

A tetranuclear copper complex $\mathbf{6}$ could be isolated directly from the blue aqueous solution obtained after ion-exchange chromatography (see above). The protonation state of 6 relates it to the species $\left[\mathrm{Cu}_{2} \mathrm{~L}\right]^{3+}$ that predominates in solution around neutral $\mathrm{pH}$, i.e. the pyrazole is deprotonated but no additional hydroxide is present. The absorption maximum in the UV/vis spectrum at $656 \mathrm{~nm}$ (shifted in comparison to $663 \mathrm{~nm}$ due to the presence of $\mathrm{NaNO}_{3}$ in solution) confirms the close relation between 6 and $\left[\mathrm{Cu}_{2} \mathrm{~L}\right]^{3+}$. The molecular structure of the cation of $\mathbf{6}$ is shown in Figure 31. 




Figure 31. Molecular structure of the cation of 6 .

Table 9. Selected atom distances $(\AA)$ and angles $\left(^{\circ}\right)$ for complex 6.

\begin{tabular}{|c|c|c|c|}
\hline \multicolumn{4}{|c|}{ Distances } \\
\hline Cu1-N1 & $2.020(2)$ & $\mathrm{Cu} 2-\mathrm{O} 2$ & $1.996(2)$ \\
\hline $\mathrm{Cu} 1-\mathrm{N} 2{ }^{\prime}$ & $1.949(2)$ & $\mathrm{Cu} 2-\mathrm{O} 3$ & $2.651(2)$ \\
\hline $\mathrm{Cu} 1-\mathrm{N} 3$ & $2.117(2)$ & $\mathrm{Cu} 2-\mathrm{N} 8$ & $2.111(2)$ \\
\hline $\mathrm{Cu} 1-\mathrm{N} 4$ & $2.106(2)$ & $\mathrm{Cu} 2-\mathrm{N} 9$ & $1.941(2)$ \\
\hline $\mathrm{Cu} 1-\mathrm{N} 6$ & $2.074(2)$ & $\mathrm{Cu} 2-\mathrm{N} 11$ & $1.952(2)$ \\
\hline $\mathrm{Cu} 2-\mathrm{O} 1$ & $2.222(2)$ & $\mathrm{Cu} 1 \cdots \mathrm{Cu} 1$ & $3.8674(6)$ \\
\hline \multicolumn{4}{|c|}{ Angles } \\
\hline $\mathrm{N} 1-\mathrm{Cu} 1-\mathrm{N} 2$ & $100.72(8)$ & $\mathrm{O} 1-\mathrm{Cu} 2-\mathrm{O} 2$ & $100.31(8)$ \\
\hline $\mathrm{N} 1-\mathrm{Cu} 1-\mathrm{N} 3$ & 79.93(8) & $\mathrm{O} 1-\mathrm{Cu} 2-\mathrm{N} 8$ & $96.23(8)$ \\
\hline $\mathrm{N} 1-\mathrm{Cu} 1-\mathrm{N} 4$ & $119.64(8)$ & $\mathrm{O} 1-\mathrm{Cu} 2-\mathrm{N} 9$ & $92.41(9)$ \\
\hline $\mathrm{N} 1-\mathrm{Cu} 1-\mathrm{N} 6$ & $119.71(8)$ & $\mathrm{O} 1-\mathrm{Cu} 2-\mathrm{N} 11$ & $88.62(8)$ \\
\hline $\mathrm{N} 2{ }^{\prime}-\mathrm{Cu} 1-\mathrm{N} 3$ & $174.15(8)$ & $\mathrm{O} 2-\mathrm{Cu} 2-\mathrm{N} 8$ & $163.40(7)$ \\
\hline $\mathrm{N} 2{ }^{\prime}-\mathrm{Cu} 1-\mathrm{N} 4$ & $95.70(8)$ & $\mathrm{O} 2-\mathrm{Cu} 2-\mathrm{N} 9$ & $95.77(8)$ \\
\hline $\mathrm{N} 2{ }^{\prime}-\mathrm{Cu} 1-\mathrm{N} 6$ & $103.90(8)$ & $\mathrm{O} 2-\mathrm{Cu} 2-\mathrm{N} 11$ & $99.77(8)$ \\
\hline $\mathrm{N} 3-\mathrm{Cu} 1-\mathrm{N} 4$ & $79.04(8)$ & N8-Cu2-N9 & $82.03(8)$ \\
\hline N3-Cu1-N6 & $80.59(8)$ & $\mathrm{N} 8-\mathrm{Cu} 2-\mathrm{N} 11$ & $81.97(8)$ \\
\hline N4-Cu1-N6 & $111.51(8)$ & N9-Cu2-N11 & $163.98(9)$ \\
\hline
\end{tabular}


Unexpectedly, X-ray crystallographic analysis of $\mathbf{6}$ revealed a dimeric arrangement of two $\left\{\mathrm{Cu}_{2} \mathrm{~L}\right\}$ subunits that features a central bis(pyrazolato) bridged dicopper(II) core. These two copper(II) ions have an almost perfect trigonal bipyramidal $\left\{\mathrm{N}_{5}\right\}$ coordination environment $(\tau$ $=0.91)$, where one of the apical $\mathrm{Cu}-\mathrm{N}$ bonds is slightly elongated at $d(\mathrm{Cu} 1-\mathrm{N} 3)=2.12 \AA$. The remaining ligand side arms are dangling and host the other two copper ions, whose coordination spheres consist of three side arm $\mathrm{N}$ atoms and one water molecule. An overall square pyramidal geometry of those outer metal ions is completed by an O-bound nitrate, but taking into account the additional weak interaction of the metal with a second $\mathrm{O}$ atom of the nitrate $(\mathrm{Cu} 2-\mathrm{O} 3=2.65 \AA)$ increases the coordination number to six and extends the coordination sphere to a strongly Jahn-Teller-distorted octahedron.

Acidification of the blue solution of 6 obtained after ion-exchange chromatography (see above) to $\sim \mathrm{pH} 4$ by the addition of aqueous $\mathrm{HClO}_{4}$ yielded crystalline material of complex 7 that relates to the solution species $\left[\mathrm{Cu}_{2} \mathrm{LH}\right]^{4+}$. As was already concluded from the titration data, 7 has a non-deprotonated pyrazole. The molecular structure of its cation is shown in Figure 32.

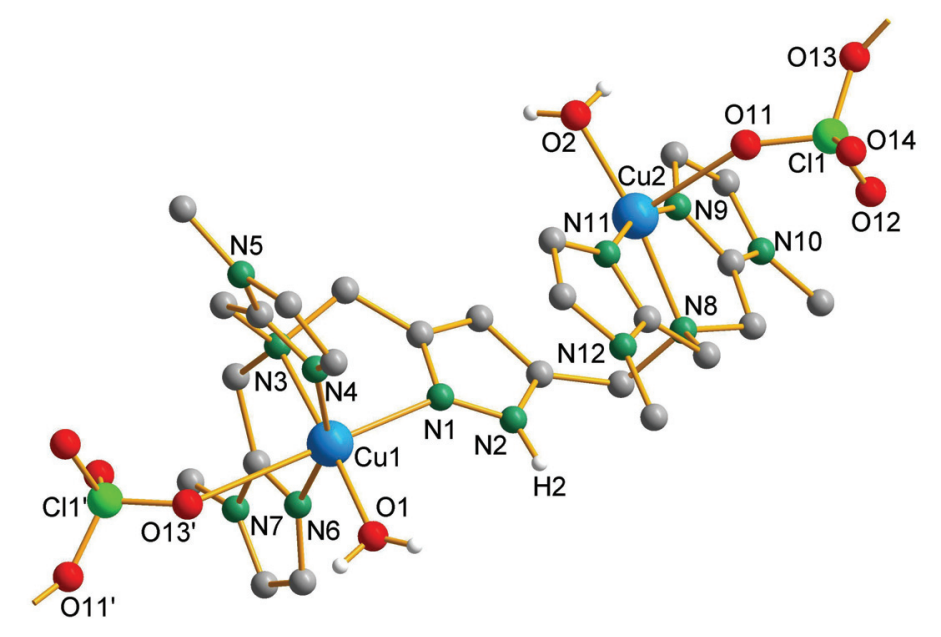

Figure 32. Molecular structure of the cation of 7. 
Table 10. Selected atom distances $(\AA)$ and angles $\left(^{\circ}\right)$ for complex 7.

\begin{tabular}{llll}
\hline \multicolumn{3}{c}{ Distances } \\
\hline $\mathrm{Cu} 1-\mathrm{O} 1$ & $1.990(3)$ & $\mathrm{Cu} 2-\mathrm{O} 2$ & $1.955(3)$ \\
$\mathrm{Cu} 1-\mathrm{O} 13$ & $2.761(4)$ & $\mathrm{Cu} 2-\mathrm{O} 11$ & $2.398(4)$ \\
$\mathrm{Cu} 1-\mathrm{N} 1$ & $2.244(4)$ & $\mathrm{Cu} 2-\mathrm{N} 8$ & $2.076(3)$ \\
$\mathrm{Cu} 1-\mathrm{N} 3$ & $2.146(3)$ & $\mathrm{Cu} 2-\mathrm{N} 9$ & $1.945(4)$ \\
$\mathrm{Cu} 1-\mathrm{N} 4$ & $1.946(4)$ & $\mathrm{Cu} 2-\mathrm{N} 11$ & $1.957(4)$ \\
$\mathrm{Cu} 1-\mathrm{N} 6$ & $1.945(4)$ & $\mathrm{Cu} 1 \cdots \mathrm{Cu} 2$ & $6.7645(8)$ \\
\hline & & Angles & $86.6(2)$ \\
\hline $\mathrm{O} 1-\mathrm{Cu} 1-\mathrm{N} 1$ & $102.1(1)$ & $\mathrm{O} 2-\mathrm{Cu} 2-\mathrm{O} 11$ & $172.0(2)$ \\
$\mathrm{O} 1-\mathrm{Cu} 1-\mathrm{Cu} 2-\mathrm{N} 8$ & $99.6(2)$ \\
$\mathrm{O} 1-\mathrm{Cu} 1-\mathrm{N} 4$ & $177.1(2)$ & $\mathrm{O} 2-\mathrm{Cu} 2-\mathrm{N} 9$ & $96.0(2)$ \\
$\mathrm{O} 1-\mathrm{Cu} 1-\mathrm{N} 6$ & $98.7(2)$ & $\mathrm{O} 2-\mathrm{Cu} 2-\mathrm{N} 11$ & $101.1(2)$ \\
$\mathrm{N} 1-\mathrm{Cu} 1-\mathrm{N} 3$ & $96.0(2)$ & $\mathrm{O} 11-\mathrm{Cu} 2-\mathrm{N} 8$ & $93.4(2)$ \\
$\mathrm{N} 1-\mathrm{Cu} 1-\mathrm{N} 4$ & $80.7(1)$ & $\mathrm{O} 11-\mathrm{Cu} 2-\mathrm{N} 9$ & $88.8(2)$ \\
$\mathrm{N} 1-\mathrm{Cu} 1-\mathrm{N} 6$ & $103.2(2)$ & $\mathrm{O} 11-\mathrm{Cu} 2-\mathrm{N} 11$ & $81.9(2)$ \\
$\mathrm{N} 3-\mathrm{Cu} 1-\mathrm{N} 4$ & $92.4(2)$ & $\mathrm{N} 8-\mathrm{Cu} 2-\mathrm{N} 9$ & $82.5(2)$ \\
$\mathrm{N} 3-\mathrm{Cu} 1-\mathrm{N} 6$ & $80.9(1)$ & $\mathrm{N} 8-\mathrm{Cu} 2-\mathrm{N} 11$ & $164.4(2)$ \\
$\mathrm{N} 4-\mathrm{Cu} 1-\mathrm{N} 6$ & $83.4(1)$ & $\mathrm{N} 9-\mathrm{Cu} 2-\mathrm{N} 11$ & \\
\hline
\end{tabular}

Complex 7 can be described as one-half of $\mathbf{6}$, since protonation of the pyrazolate splits the central bis(pyrazolato) dicopper core and leaves only one of the copper ions coordinated to the non-bridging pyrazole fragment. $\mathrm{Cul}$ is found in an octahedral coordination environment, ligated equatorially by three $\mathrm{N}$-donors from the side arm of $\mathbf{H L}^{4}$ and an $\mathrm{O}$ atom from a water molecule. Axial positions are occupied by the distant N-donor from pyrazole (2.24 $\AA$ ) and a perchlorate counter-anion at an even greater distance $(2.76 \AA)$, typical for a Jahn-Teller elongated situation. The second metal is again nested in the dangling chelate arm compartment and is five-coordinate $(\tau=0.13)$ with an additional water in the equatorial and a perchlorate-O in the axial position. A three-dimensional network is built up by H-bonds between coordinated water molecules, perchlorate anions and the NH-unit of the pyrazole. A one-dimensional chain is represented in Figure 33, where the dicopper pyrazole bridged compartments are connected by a $\mu$-bidentate perchlorate bridge via two copper ions from the different units (Cu1-O13' (2.76 ̊), Cu2-O11 (2.39 ̊). 


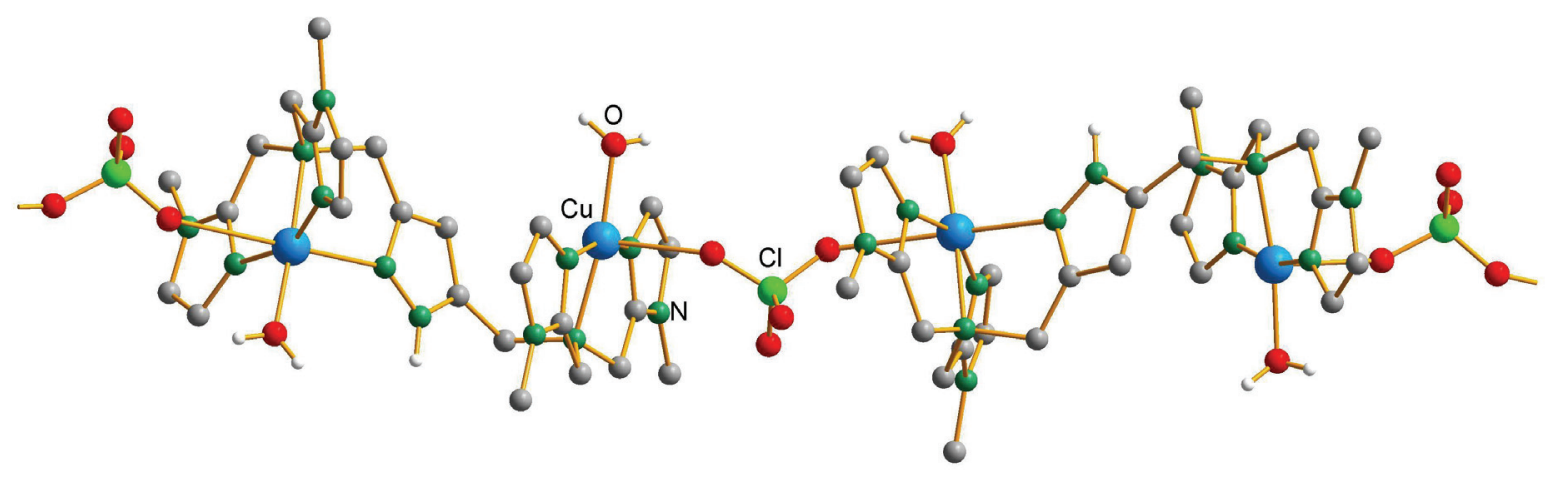

Figure 33. Schematic representation of the one-dimensional chain of 7.

\subsubsection{Spectroscopic properties of the complexes and equilibria in solution}

UV/vis spectra of the three complexes 5a, 6, and 7 have been recorded in $\mathrm{MeOH} / \mathrm{MeCN}(5: 2)$ solution as well as in the solid state (diffuse reflectance). Table 7 (see page 52) compares findings for the ligand-field bands for the species in aqueous solution at different $\mathrm{pH}$ as well as for the isolated compounds. Absorption maxima in the diffuse reflectance spectra are in accordance with the coordination geometries observed crystallographically, i.e. trigonal bipyramidal for 5a (broad $d-d$ absorption at $\sim 950 \mathrm{~nm}$ ), tetragonal for 7 (weak ligand field band at $\sim 690 \mathrm{~nm}$ ) and the presence of both signatures in the case of 6 ( $\lambda_{\max }$ is also affected by the type of donor atoms). Similar spectra were observed for each complex in $\mathrm{MeOH} / \mathrm{MeCN}$ solution, implying that no major structural changes occur upon redissolving the crystalline solids. The same is true for species in aqueous solution at the respective $\mathrm{pH}$ except for $\mathbf{6}$. In the latter system the broad $d$ - $d$ absorption at $\sim 920 \mathrm{~nm}$ seen in the diffuse reflectance spectra and in $\mathrm{MeOH} / \mathrm{MeCN}$ solution is strongly diminished in aqueous solution around neutral $\mathrm{pH}$. Since a trigonal bipyramidal situation (characterized by the $d$ - $d$ band at $\sim 920 \mathrm{~nm}$ ) is observed for the central bis(pyrazolato) bridged dicopper(II) core in $\mathbf{6}$, it is reasonable to assume that in aqueous solution the dimeric aggregate largely breaks apart to give bimetallic species $\left[\mathrm{Cu}_{2} \mathrm{~L}\left(\mathrm{H}_{2} \mathrm{O}\right)_{\mathrm{x}}\right]^{3+}$. It should be noted that pH-potentiometry cannot differentiate between bimetallic $\left[\mathrm{Cu}_{2} \mathrm{~L}\right]^{3+}$ and its dimeric aggregate $\left[\left(\mathrm{Cu}_{2} \mathrm{~L}\right)_{2}\right]^{6+}$.

Equilibria in solution between the three different complexes was monitored by UV/vis spectroscopy upon titration with a base, starting at $\mathrm{pH}$ 4.66. Dicopper complex 7 was dissolved in $0.1 \mathrm{M} \mathrm{NaNO}_{3}$ (in order to prevent precipitation of the perchlorate salt $\mathbf{5 a}$ at higher $\mathrm{pH}$ ), with only $\left[\mathrm{Cu}_{2} \mathrm{LH}\right]^{4+}$ present in solution at $\mathrm{pH}$ 4.66. After stepwise addition of base $(\mathrm{NaO} t \mathrm{Bu})$, the initial dicopper complex was fully converted into $\left[\mathrm{Cu}_{2} \mathrm{~L}\right]^{3+}$ at $\mathrm{pH} 6.55$. 
Further addition of base led to subsequent formation of $\left[\mathrm{Cu}_{2} \mathrm{LH}_{-1}\right]^{2+}$ (which is suggested to represent the dinuclear species $\mathbf{5} \mathbf{b}^{\prime}$ with a $\mathrm{HO} \cdots \mathrm{HOH}$ bridge). This becomes the only species at $\mathrm{pH} 9.40$ (Figure 34).



Figure 34. UV/vis titration of a solution of 7 in $0.1 \mathrm{M} \mathrm{NaNO}_{3}$ with $\mathrm{NaO} t \mathrm{Bu}$ between $\mathrm{pH} 6.55$ (a) and $\mathrm{pH} 9.40$ (b).

The appropriate amount of base necessary to convert tetranuclear 6 into dicopper(II) complex 5b was established by titration of complex 6 with base $(\mathrm{KO} t \mathrm{Bu})$ in $\mathrm{MeOH} / \mathrm{MeCN}(5: 2)$ solution. The concentration of complex 6 was varied in the range from 0.0059 to $0.0027 \mathrm{~mol}$ $\mathrm{L}^{-1}$. Titrations were performed by varying the ratio complex:base from $0.1: 1.0$ to $2.01: 1.0$. Complete conversion of tetranuclear $\mathbf{6}$ into dinuclear $\mathbf{5 b}$ was observed after addition of two equivalents of $\mathrm{KO} t \mathrm{Bu}$, consistent with the structural data: two equivalents of base should be required to split the dimeric species into its bimetallic $\left\{\mathrm{Cu}_{2} \mathrm{~L}\right\}$ constituents, concomitant with formation of the $\mathrm{MeO} \cdots \mathrm{HOMe}$ bridge (Figure 35). 


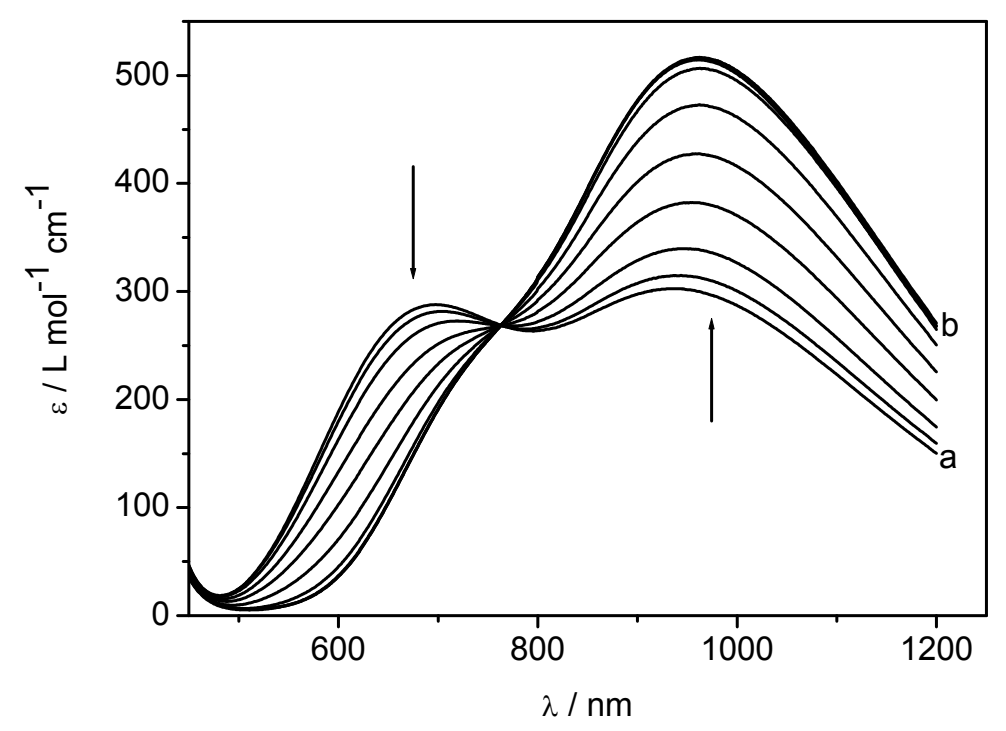

Figure 35. UV/vis titration of a solution of 6 in $\mathrm{MeOH} / \mathrm{MeCN}(5: 2)$ with $\mathrm{KO} t \mathrm{Bu}$ : solution of 6 without $\mathrm{KO} t \mathrm{Bu}(\mathrm{a})$ and with 2 eq. $\mathrm{KO} t \mathrm{Bu}(\mathrm{b})$.

During the course of these studies, an additional concentration dependence was observed for $\mathbf{6}$ (Figure 36), suggesting an equilibrium between the tetranuclear complex and a putative dinuclear species $\mathbf{6}^{\prime}$ around neutral $\mathrm{pH}$. A concentrated solution of complex 6 (0.01 M in $\mathrm{MeOH} / \mathrm{MeCN}(5: 2))$ showed two bands at $689 \mathrm{~nm}$ and $923 \mathrm{~nm}$, in accordance with the two types of copper ions found in the solid state (square pyramidal and trigonal bipyramidal). Dilution of this solution causes a shift of the absorption envelope from 923 to $962 \mathrm{~nm}$ and from 689 to $710 \mathrm{~nm}$, with concomitant increase in intensity for the former and decrease in intensity for the latter band. Spectral features for $\mathbf{6}^{\mathbf{\prime}}$ are reminiscent of those seen for 5a. The equilibrium thus induces a structural reorganization of the copper centers from square pyramidal to trigonal bipyramidal upon dilution, which is likely associated with dissociation of the tetranuclear complex into bimetallic species $\left[\mathrm{Cu}_{2} \mathrm{~L}\right]^{3+}\left(6^{\prime}\right)$. 


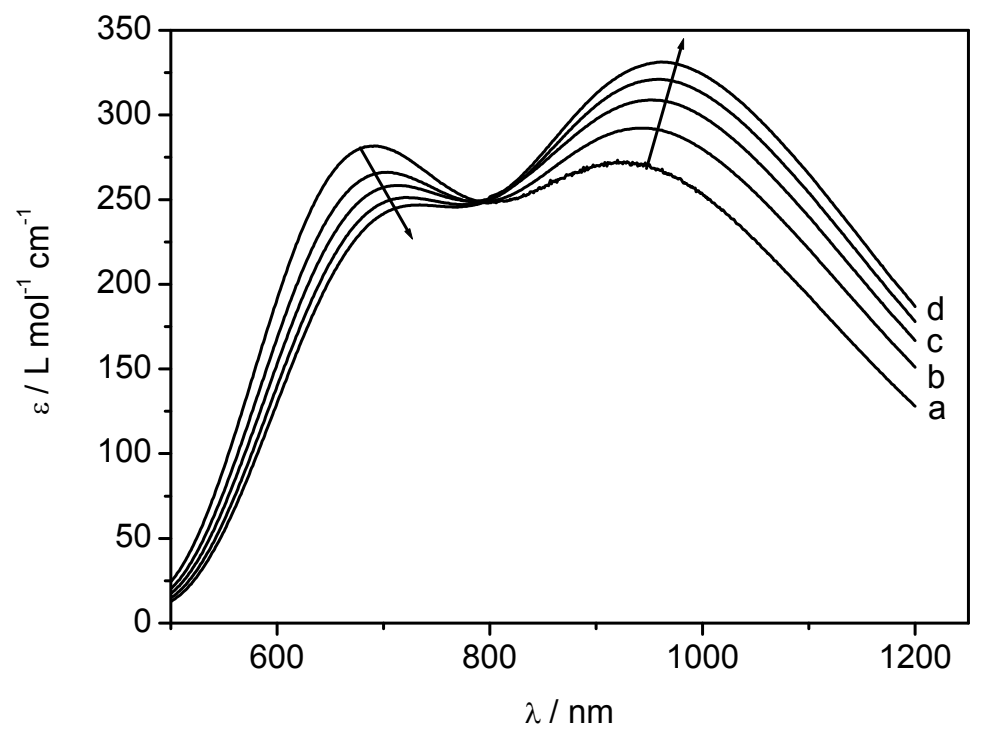

Figure 36. UV/vis spectra of 6 at different concentrations: $0.01 \mathrm{M}(\mathrm{a}) ; 0.005 \mathrm{M}(\mathrm{b}) ; 0.003 \mathrm{M}$ (c); $0.002 \mathrm{M}$ (d) in $\mathrm{MeOH} / \mathrm{MeCN}$ (5:2).

Above described overall equilibria between the three different complexes in $0.1 \mathrm{M}$ aqueous solution of $\mathrm{NaNO}_{3}$ is depicted in Scheme 27.

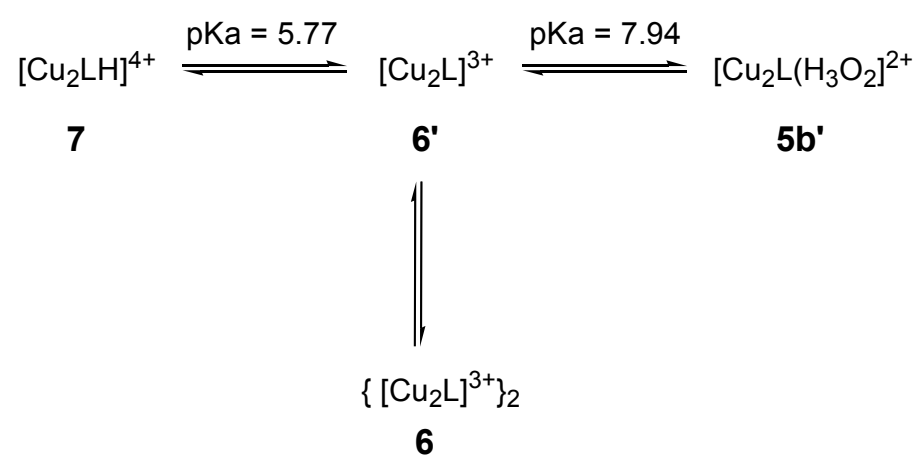

Scheme 27. Schematic representation of the overall equilibria between three complexes in 0.1 $\mathrm{M} \mathrm{NaNO}_{3}$ solution: dinuclear species 7 at $\mathrm{pH}=4.66$; tetranuclear species 6 at $\mathrm{pH}=6.55$; dinuclear species $\mathbf{6}$ ' formed upon dissociation of $\mathbf{6}$ at neutral $\mathrm{pH}$; dinuclear species $\mathbf{5} \mathbf{b}^{\mathbf{\prime}}$ at $\mathbf{p H}=$ 9.40 with a $\mathrm{HO} \cdots \mathrm{HOH}$ bridge. 


\subsubsection{Magnetic properties}

Magnetic susceptibilities for powdered samples of 5a, 6, and 7 were measured at two different magnetic fields $(2000 \mathrm{G}$ and $5000 \mathrm{G})$ in a temperature range from $2.0 \mathrm{~K}$ to $295 \mathrm{~K}$. No significant field dependence was observed for any of the complexes. The temperature dependence of the magnetic susceptibility $\chi_{M}$ and of the product $\chi_{M} T$ for the three distinct systems is depicted in Figure 37.

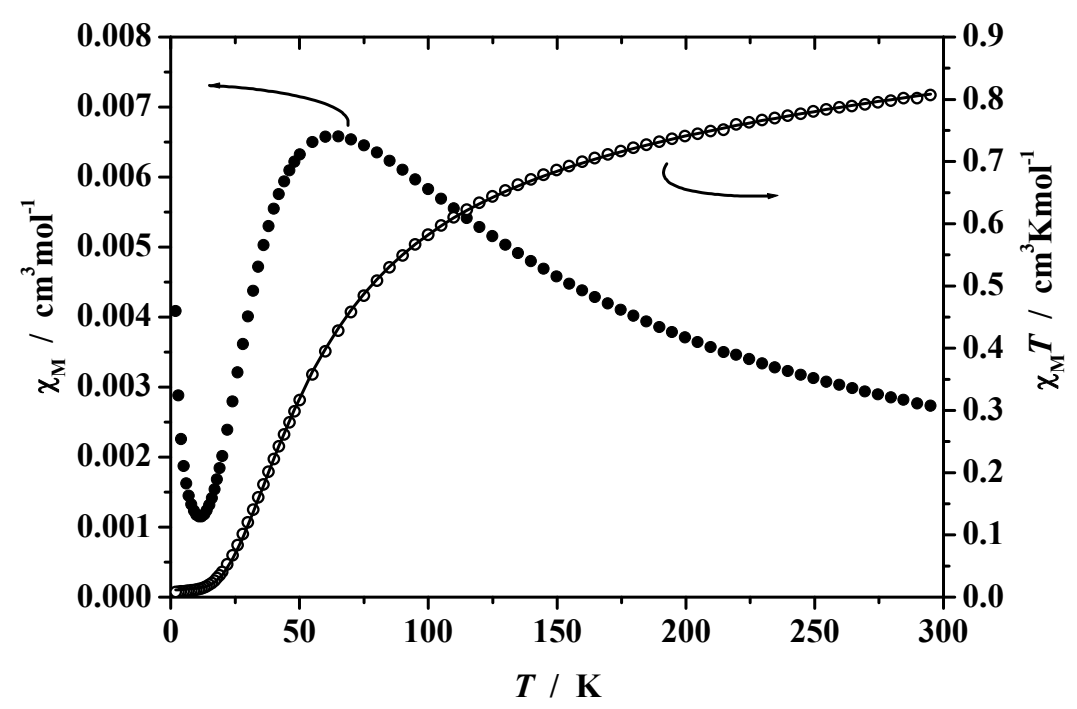



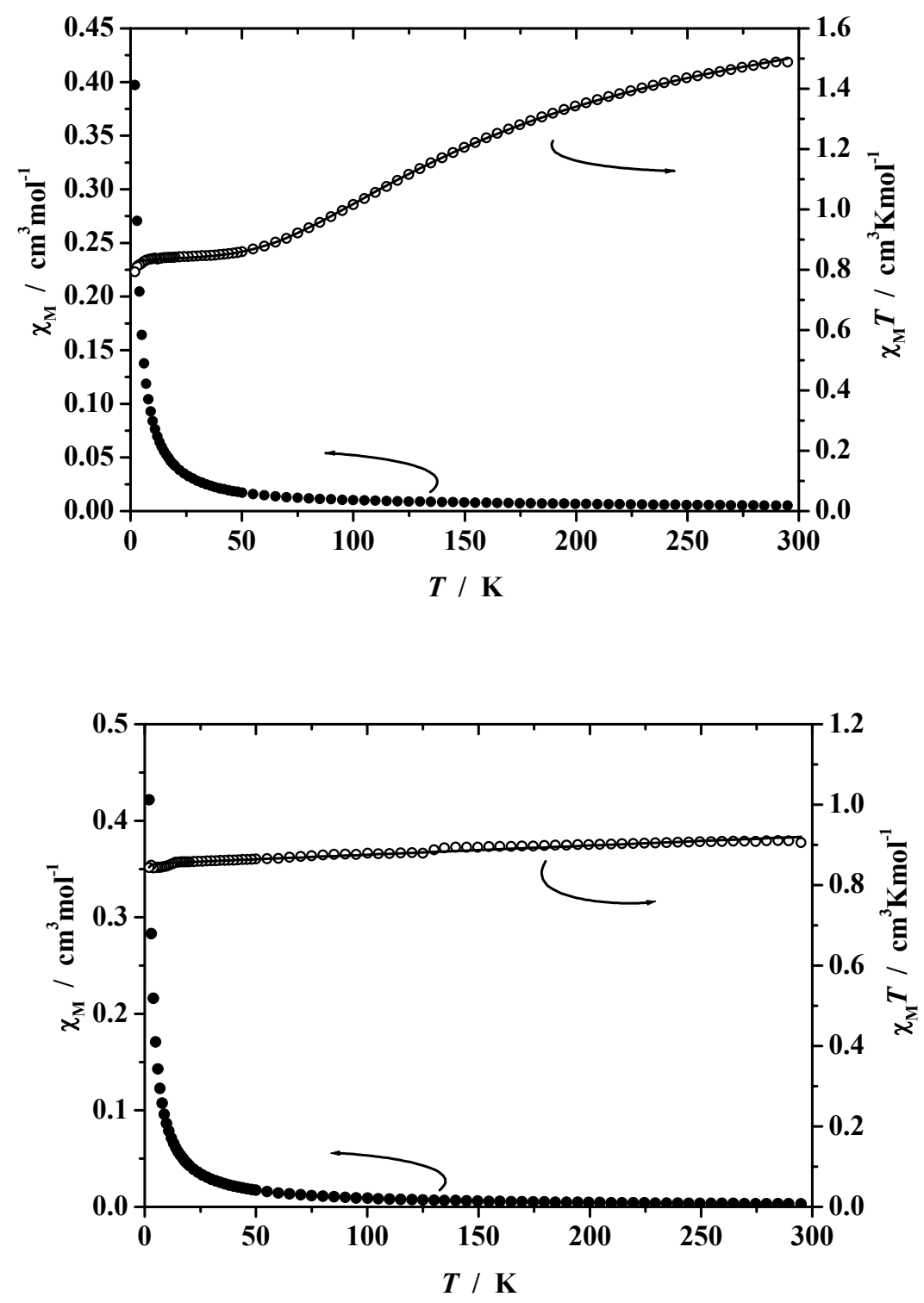

Figure 37. Plots of $\chi_{M}$ (solid circles) and $\chi_{M} T$ (open circles) versus temperature for 5a (top), 6 (middle) and 7 (bottom) at $5000 \mathrm{G}$; the solid black lines represent the calculated curve fits (see text).

The observed $\chi_{M} T$ value at $295 \mathrm{~K}$ for dicopper complex 7 is $0.91 \mathrm{~cm}^{3} \cdot \mathrm{K}^{\prime} \cdot \mathrm{mol}^{-1}\left(2.63 \mu_{\mathrm{B}}\right)$, which indicates the presence of two uncoupled copper(II) ions $\left(2.62 \mu_{\mathrm{B}}\right.$ for $\left.g=2.14\right)$. In contrast to 5a and $\mathbf{6}, \chi_{M} T$ remains almost constant over a wide temperature range and shows only a slight linear decrease when the temperature is lowered to $2 \mathrm{~K}$, which is a signature for complexes without magnetic interaction.

Experimental data for both dinuclear complexes 5a and 7 were modelled by using a fitting procedure to the appropriate Heisenberg-Dirac-van-Vleck (HDvV) spin Hamiltonian for 
isotropic exchange coupling and Zeeman splitting, equation (1). ${ }^{118}$ A Curie-Weiss behaved paramagnetic impurity $(\rho)$ with spin $S=1 / 2$ and temperature-independent paramagnetism (TIP) were included according to $\chi=(1-\rho) \cdot \chi+\rho \cdot \chi_{\text {mono }}+T I P .{ }^{119}$ Susceptibility data for the tetranuclear complex 6 were treated in a model that assumes an exchange-coupled bis $(\mu-$ pyrazolato) bridged dicopper(II) core according to equation (1), but with an additional singleion Curie term for two well-separated non-interacting copper(II) ions (with $g_{2}$ ). Calculated curve fits are shown as solid lines in Figure 37, and best fit parameters for all complexes are summarized in Table 11.

$$
H=-2 J \vec{S}_{1} \vec{S}_{2}+g \mu_{B}\left(\vec{S}_{1}+\vec{S}_{2}\right) \vec{B}
$$

Table 11. Best fit parameters of magnetic data analyses for complexes 5a, 6 and 7.

\begin{tabular}{cccc}
\hline complex & $\mathbf{5 a}$ & $\mathbf{6}$ & $\mathbf{7}$ \\
\hline$g_{l}$ & 2.07 & 2.06 & 2.14 \\
$g_{2}$ & - & 2.11 & - \\
$J, \mathrm{~cm}^{-1}$ & -35.3 & -100.6 & 0 \\
$\rho, \%$ & 2.9 & 1.9 & 0.3 \\
$T I P, \mathrm{~cm}^{3} \cdot \mathrm{mol}^{-1}$ & $3.09 \mathrm{E}-4$ & $4.13 \mathrm{E}-4$ & $2.27 \mathrm{E}-4$ \\
\hline
\end{tabular}

The differences in the magnetic properties for 5a, $\mathbf{6}$ and 7 can be rationalized on the basis of the crystallographic findings. Tetranuclear $\mathbf{6}$ has two different types of copper ions: two metal ions constitute the bis( $\mu$-pyrazolato $)$ bridged core $\left(d\left(\mathrm{Cu} 1^{\cdots} \mathrm{Cu} 1^{\prime}\right)=3.86 \AA\right)$ and two further ones are coordinated to the remaining ligand side arms and are well separated from the central core $\left(d\left(\mathrm{Cu} 1 \cdots \mathrm{Cu} 2^{\prime}\right)=8.31 \AA\right)$. Antiferromagnetic coupling is observed only within the pyrazolate-bridged core, while the constant value of $\chi_{M} T\left(0.84 \mathrm{~cm}^{3} \mathrm{Kmol}^{-1}\right)$ below $50 \mathrm{~K}$ confirms the presence of two additional uncoupled copper(II) ions. Two different $g$ values (2.06 and 2.11) were observed for complex 6, in agreement with the proposed model. The magnitude of the antiferromagnetic interaction in $\mathbf{5 a}$ containing a single pyrazolate bridge is $-35.3 \mathrm{~cm}^{-1}$, which is much lower than for the central part of complex $6\left(-100.6 \mathrm{~cm}^{-1}\right)$ with its doubly pyrazolato-bridged dicopper(II) core. It can be safely concluded that the primary pathway for the magnetic interaction is through the pyrazolate bridges, whereas coupling through the H-bonded $\mathrm{MeO} \cdots \mathrm{HOMe}$ moiety in 5a should be negligible. The value for the antiferromagnetic interaction in $\mathbf{6}$, however, is still relatively small compared to data for 
various other bis( $\mu$-pyrazolato) bridged dicopper(II) compounds. ${ }^{120,121,122}$ This can be ascribed to the trigonal bipyramidal coordination geometry imposed by the tripodal tetradentate $\left\{\mathrm{N}_{4}\right\}$ ligand compartments of $\mathbf{H L}^{4}$ and the resulting orientation of the magnetic $\mathrm{d}_{\mathrm{z} 2}$ orbital of each metal ion, which represents a quite rare situation in copper pyrazolate chemistry. ${ }^{123}$ The bridging pyrazolate groups in $\mathbf{6}$ are positioned in such a way that one $\mathrm{N}$ atom occupies an axial site on one copper while the other $\mathrm{N}$ atom occupies an equatorial site on the second copper, and consequently the overlap of the magnetic $\mathrm{d}_{\mathrm{z} 2}$ orbitals with ligand orbitals of the metal xy plane is relatively weak. In contrast, many other known dicopper(II) complexes that are spanned by two pyrazolato bridges feature square planar or tetragonal metal ions where the magnetic $d_{x 2-y 2}$ orbitals are located within the plane of the bridging heterocycles. ${ }^{120,123}$ This latter situation establishes a much more efficient coupling pathway and $J$ values in the order of $-200 \mathrm{~cm}^{-1}$. It should be noted that additional parameters such as the deviation from co-planarity of the pyrazolate planes, ${ }^{124}$ the $\mathrm{Cu}-\mathrm{N}-\mathrm{N}$ angle, ${ }^{125}$ the bending angles $\delta_{\text {pz-bend }}$ (representing the dihedral angle of the pyrazolate plane relative to the $\mathrm{Cu}-\mathrm{N}-\mathrm{N}-$ $\mathrm{Cu}$ plane), ${ }^{123}$ and possibly the terminal donor atoms have been found to also contribute to the strength of the antiferromagnetic coupling in bis(pyrazolato) bridged dicopper(II) scaffolds.

The magnetic behaviour of dicopper complex 7, which does not feature a pyrazolate-bridging motif but a large $\mathrm{Cu} \cdots \mathrm{Cu}$ distance of $6.76 \AA$, is in accordance with the absence of any efficient exchange pathway and the lack of any significant coupling between the two metal ions. The slight linear decrease of $\chi_{M} T$ upon lowering the temperature to $2 \mathrm{~K}$ in this case is merely due to the $\operatorname{TIP}\left(2.27 \mathrm{E}-4 \mathrm{~cm}^{3} \mathrm{~mol}^{-1}\right)$.

\subsubsection{Cyclovoltammetry}

Cyclovoltammetric measurements on dicopper(II) complex 5a revealed only one irreversible reduction process at $E_{\mathrm{p}}{ }^{\mathrm{Red}}=-1.3 \mathrm{~V}$ versus the $\mathrm{Cp}_{2} \mathrm{Fe} / \mathrm{Cp}_{2} \mathrm{Fe}^{+}$couple. The cyclic voltammogram of $\mathbf{5 a}$ is depicted in Figure 38. 


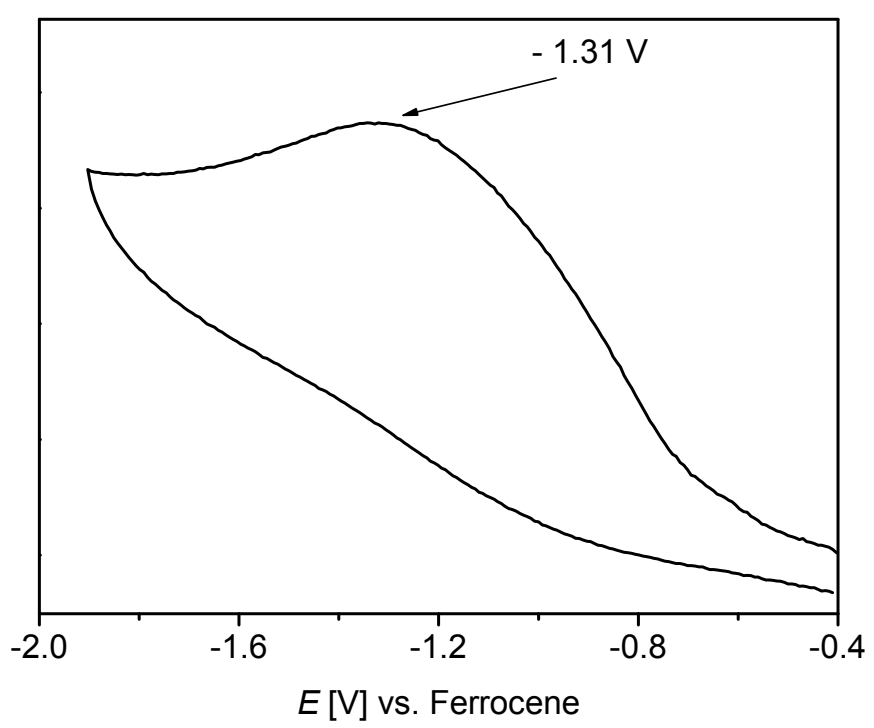

Figure 38. Cyclic voltammogram of 5a in $\operatorname{MeCN}\left(0.1 \mathrm{M} \mathrm{N}^{n} \mathrm{Bu}_{4} \mathrm{PF}_{6}, 100 \mathrm{mV} \cdot \mathrm{s}^{-1}\right)$; peak potentials in Volts versus $\mathrm{Cp}_{2} \mathrm{Fe} / \mathrm{Cp}_{2} \mathrm{Fe}^{+}$couple.

The reduction occurred only at low potential, which illustrates the significant stability of the dicopper(II) species. ${ }^{74,108}$ Moreover, the irreversible nature of the reduction most likely is caused by the drastic differences between copper(II) and copper(I) regarding their structural and electronic requirements. Electrochemical production of the copper(I) species is presumably supported by a structural reorganisation relative to the initial dicopper(II) species, i.e. dissociation of the bridging $\mathrm{MeO} \cdots \mathrm{HOMe}$ unit, which possesses a very low affinity to copper(I) ions. The preferences in coordination geometry for the copper(I) ion formed upon reduction could also modify the overall structural motif found in $\mathbf{5 a}$ by disruption of the strongly chelating, dinucleating character of the ligand as observed in the dicopper(II) species, resulting in an expulsion of one of the side arms to generate a preferable coordination pocket for the copper(I) ion, as for example observed in complex 9 (see section 7.6.4). However, the irreversible nature of the electrochemically induced redox-process clearly does not affect the activity of dicopper(II) complex $\mathbf{5 a}$ as an oxidation catalyst, in particular for the oxidative CC coupling of TMP (see section 6.3). The single observable reduction peak can be assigned to a $2 \mathrm{e}^{-}$reduction of the $\mathrm{Cu}^{\mathrm{II}} \mathrm{Cu}^{\mathrm{II}}$ species to yield the corresponding $\mathrm{Cu}^{\mathrm{I}} \mathrm{Cu}^{\mathrm{I}}$ analogue or to only $1 \mathrm{e}^{-}$reduction of the initial dicopper(II) species resulting in the formation of a mixed-valence $\mathrm{Cu}^{\mathrm{I}} \mathrm{Cu}^{\mathrm{II}}$ species. The oxidation process is taking place at high potentials (not shown in the Figure $38, \sim 1.7 \mathrm{~V}$ ) and occurs most probably in concert with oxidation of the solvent used for these measurements. 


\subsection{Characterization of a copper complex with the new ligand $\mathrm{HL}^{5}$}

The new ligand $\mathbf{H L}^{\mathbf{5}}$, based on 4-phenyl pyrazole as the core framework, was prepared as the first representative of a new family of substituted pyrazole-based compartmental ligands featuring an arylated pyrazole ring. This substitution of a phenyl group instead of a proton is expected to significantly change the solubility of the copper complexes derived from ligands featuring this building block and at the same time influence the properties of the molecule (packing) in the solid state and hopefully enhance the isolation of crystalline samples.

The standard synthetic route was applied for the synthesis of copper complexes. Addition of two equivalents of $\mathrm{Cu}\left(\mathrm{NO}_{3}\right)_{2}$ to a $\mathrm{MeOH} / \mathrm{MeCN}$ solution containing $\mathbf{H L}^{\mathbf{5}}$ and two equivalents of base $(\mathrm{KO} t \mathrm{Bu})$ produced a green reaction mixture, from which single crystals were obtained by diffusion of $\mathrm{Et}_{2} \mathrm{O}$ that were suitable for an X-ray crystallographic analysis. The resultant molecular structure of the cation of $\mathbf{8}$ is depicted in Figure 39.



Figure 39. Molecular structure of the cation of $\mathbf{8}$.

Surprisingly, formation of the kindred tetranuclear species took place instead of the expected dinuclear. This tetranuclear complex 8 possesses two independent dinuclear units that are bridged by two $\mu-\mathrm{OH}$ ligands. All four copper atoms are ligated by four $\mathrm{N}$ atoms, i.e. one from the pyrazolate-unit, two from the imidazoles in the side arm and the tertiary $\mathrm{N}$ atom of the ligand backbone, and by the $\mathrm{O}$ atom from the bridging $\mathrm{OH}$ unit. This ligation results in an 
almost perfect trigonal bipyramidal geometry for $\mathrm{Cu} 3(\tau=0.95)$ and $\mathrm{Cu} 4(\tau=0.92)$ as well as for the other two copper atoms $(\tau=0.95)($ Table 12$)$.

Table 12. Selected intramolecular distances $(\AA)$ and angles $\left(^{\circ}\right)$ for complex 8 .

\begin{tabular}{|c|c|c|c|}
\hline \multicolumn{4}{|c|}{ Distances } \\
\hline $\mathrm{Cu}(1)-\mathrm{O}(1)$ & $1.911(3)$ & $\mathrm{Cu}(3)-\mathrm{N}(24)$ & $2.123(3)$ \\
\hline $\mathrm{Cu}(1)-\mathrm{N}(1)$ & $1.975(4)$ & $\mathrm{Cu}(3)-\mathrm{N}(23)$ & $2.130(3)$ \\
\hline $\mathrm{Cu}(1)-\mathrm{N}(6)$ & $2.011(4)$ & $\mathrm{Cu}(4)-\mathrm{O}(1)$ & $1.895(3)$ \\
\hline $\mathrm{Cu}(1)-\mathrm{N}(3)$ & $2.136(3)$ & $\mathrm{Cu}(4)-\mathrm{N}(22)$ & $2.041(3)$ \\
\hline $\mathrm{Cu}(1)-\mathrm{N}(4)$ & $2.136(4)$ & $\mathrm{Cu}(4)-\mathrm{N}(29)$ & $2.043(4)$ \\
\hline $\mathrm{Cu}(2)-\mathrm{O}(2)$ & $1.935(3)$ & $\mathrm{Cu}(4)-\mathrm{N}(31)$ & $2.090(4)$ \\
\hline $\mathrm{Cu}(2)-\mathrm{N}(9)$ & $2.032(4)$ & $\mathrm{Cu}(4)-\mathrm{N}(28)$ & $2.111(4)$ \\
\hline $\mathrm{Cu}(2)-\mathrm{N}(2)$ & $2.056(3)$ & $\mathrm{Cu}(1) \cdots \mathrm{Cu}(2)$ & $4.2895(7)$ \\
\hline $\mathrm{Cu}(2)-\mathrm{N}(11)$ & $2.063(4)$ & $\mathrm{Cu}(1) \cdots \mathrm{Cu}(3)$ & $5.4999(6)$ \\
\hline $\mathrm{Cu}(2)-\mathrm{N}(8)$ & $2.116(4)$ & $\mathrm{Cu}(1) \cdots \mathrm{Cu}(4)$ & $3.4877(7)$ \\
\hline $\mathrm{Cu}(3)-\mathrm{O}(2)$ & $1.943(3)$ & $\mathrm{Cu}(2) \cdots \mathrm{Cu}(3)$ & $3.5249(7)$ \\
\hline $\mathrm{Cu}(3)-\mathrm{N}(21)$ & $2.017(3)$ & $\mathrm{Cu}(2) \cdots \mathrm{Cu}(4)$ & $4.6024(8)$ \\
\hline $\mathrm{Cu}(3)-\mathrm{N}(26)$ & $2.062(3)$ & $\mathrm{Cu}(3) \cdots \mathrm{Cu}(4)$ & $4.3774(7)$ \\
\hline \multicolumn{4}{|c|}{ Angles } \\
\hline $\mathrm{O}(1)-\mathrm{Cu}(1)-\mathrm{N}(1)$ & 99.91(14) & $\mathrm{O}(2)-\mathrm{Cu}(3)-\mathrm{N}(21)$ & $101.19(13)$ \\
\hline $\mathrm{O}(1)-\mathrm{Cu}(1)-\mathrm{N}(6)$ & $96.26(14)$ & $\mathrm{O}(2)-\mathrm{Cu}(3)-\mathrm{N}(26)$ & $97.95(13)$ \\
\hline $\mathrm{N}(1)-\mathrm{Cu}(1)-\mathrm{N}(6)$ & $123.54(17)$ & $\mathrm{N}(21)-\mathrm{Cu}(3)-\mathrm{N}(26)$ & $120.35(14)$ \\
\hline $\mathrm{O}(1)-\mathrm{Cu}(1)-\mathrm{N}(3)$ & $173.79(14)$ & $\mathrm{O}(2)-\mathrm{Cu}(3)-\mathrm{N}(24)$ & $102.16(13)$ \\
\hline $\mathrm{N}(1)-\mathrm{Cu}(1)-\mathrm{N}(3)$ & $79.75(14)$ & $\mathrm{N}(21)-\mathrm{Cu}(3)-\mathrm{N}(24)$ & $112.90(14)$ \\
\hline $\mathrm{N}(6)-\mathrm{Cu}(1)-\mathrm{N}(3)$ & $78.96(15)$ & $\mathrm{N}(26)-\mathrm{Cu}(3)-\mathrm{N}(24)$ & $117.21(14)$ \\
\hline $\mathrm{O}(1)-\mathrm{Cu}(1)-\mathrm{N}(4)$ & $107.80(14)$ & $\mathrm{O}(2)-\mathrm{Cu}(3)-\mathrm{N}(23)$ & $177.73(13)$ \\
\hline $\mathrm{N}(1)-\mathrm{Cu}(1)-\mathrm{N}(4)$ & $111.20(15)$ & $\mathrm{N}(21)-\mathrm{Cu}(3)-\mathrm{N}(23)$ & $80.38(12)$ \\
\hline $\mathrm{N}(6)-\mathrm{Cu}(1)-\mathrm{N}(4)$ & $114.36(17)$ & $\mathrm{N}(26)-\mathrm{Cu}(3)-\mathrm{N}(23)$ & $79.82(13)$ \\
\hline $\mathrm{N}(3)-\mathrm{Cu}(1)-\mathrm{N}(4)$ & $77.95(14)$ & $\mathrm{N}(24)-\mathrm{Cu}(3)-\mathrm{N}(23)$ & $78.61(13)$ \\
\hline $\mathrm{O}(2)-\mathrm{Cu}(2)-\mathrm{N}(9)$ & 98.91(14) & $\mathrm{O}(1)-\mathrm{Cu}(4)-\mathrm{N}(22)$ & $104.47(13)$ \\
\hline $\mathrm{O}(2)-\mathrm{Cu}(2)-\mathrm{N}(2)$ & $105.83(13)$ & $\mathrm{O}(1)-\mathrm{Cu}(4)-\mathrm{N}(29)$ & $98.84(14)$ \\
\hline $\mathrm{N}(9)-\mathrm{Cu}(2)-\mathrm{N}(2)$ & $114.22(16)$ & $\mathrm{N}(22)-\mathrm{Cu}(4)-\mathrm{N}(29)$ & $117.54(14)$ \\
\hline $\mathrm{O}(2)-\mathrm{Cu}(2)-\mathrm{N}(11)$ & $97.18(15)$ & $\mathrm{O}(1)-\mathrm{Cu}(4)-\mathrm{N}(31)$ & $97.56(15)$ \\
\hline
\end{tabular}




$\begin{array}{llll}\mathrm{N}(9)-\mathrm{Cu}(2)-\mathrm{N}(11) & 121.91(15) & \mathrm{N}(22)-\mathrm{Cu}(4)-\mathrm{N}(31) & 114.46(14) \\ \mathrm{N}(2)-\mathrm{Cu}(2)-\mathrm{N}(11) & 114.07(15) & \mathrm{N}(29)-\mathrm{Cu}(4)-\mathrm{N}(31) & 118.68(16) \\ \mathrm{O}(2)-\mathrm{Cu}(2)-\mathrm{N}(8) & 175.17(14) & \mathrm{O}(1)-\mathrm{Cu}(4)-\mathrm{N}(28) & 174.82(13) \\ \mathrm{N}(9)-\mathrm{Cu}(2)-\mathrm{N}(8) & 80.17(18) & \mathrm{N}(22)-\mathrm{Cu}(4)-\mathrm{N}(28) & 80.58(13) \\ \mathrm{N}(2)-\mathrm{Cu}(2)-\mathrm{N}(8) & 78.79(15) & \mathrm{N}(29)-\mathrm{Cu}(4)-\mathrm{N}(28) & 79.55(14) \\ \mathrm{N}(11)-\mathrm{Cu}(2)-\mathrm{N}(8) & 79.44(19) & \mathrm{N}(31)-\mathrm{Cu}(4)-\mathrm{N}(28) & 79.07(16)\end{array}$

The bridging $\mathrm{OH}^{-}$ligands between two dicopper units induce an enlargement of the coppercopper distance within the independent bimetallic core to $4.60 \AA$ for $d(\mathrm{Cu} 2 \cdots \mathrm{Cu} 4)$, compared to the usual distance observed in dinuclear copper(II) complexes with related imidazole side arms (see section 5.4.2). However, the distance between two copper atoms belonging to different bimetallic subunits that are connected by a $\mu-\mathrm{OH}$ ligand is rather small $(d(\mathrm{Cu} 1 \cdots \mathrm{Cu} 4)=3.49 \AA)$.

The structural features were reflected perfectly in the corresponding UV/vis spectrum, where one $d$ - $d$ transition band for copper ions in a trigonal bipyramidal environment at $\lambda_{\max }$ of 995 $\mathrm{nm}$ was observed with the typical additional shoulder at $\lambda_{\max }$ of $726 \mathrm{~nm}$. Unfortunately, it was not possible by means of UV/vis spectroscopy to distinguish between the isolated tetranuclear species and the related dinuclear complex, which presumably forms in solution through dissociation of the former, since the dinuclear species, should have similar spectroscopic features.
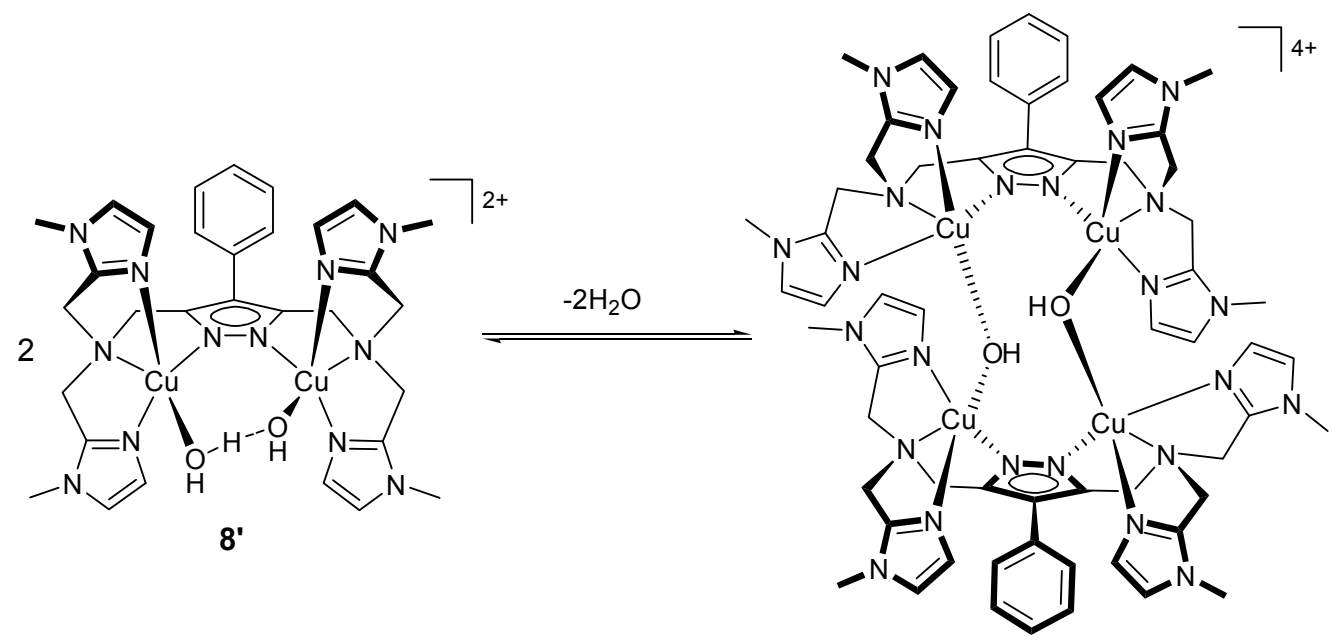

8

Scheme 28. Equilibrium in aqueous solution between tetranuclear species $\mathbf{8}$ and dinuclear species $\mathbf{8}^{\prime}$. 
The ESI spectrum of the MeCN solution of $\mathbf{8}$ confirmed the presumed dissociation process resulting in formation of dinuclear subunit $\mathbf{8}^{\prime}$ with $\mathrm{MeO}{ }^{\cdots} \mathrm{HOMe}$ or $\mathrm{HO}{ }^{\cdots} \mathrm{HOH}$ bridging motif (depending on the solvent used) within the bimetallic pocket (Scheme 28), as for example observed in 5a.The observed signals in the ESI spectrum at 703, 766 and $827 \mathrm{~m} / \mathrm{z}$ are typical for dinuclear copper(II) species, as they correspond to $\left[\mathrm{Cu}_{2} \mathrm{~L}^{5}\right]^{+},\left[\mathrm{Cu}_{2} \mathrm{~L}^{5}\left(\mathrm{NO}_{3}\right)\right]^{+}$and $\left[\mathrm{Cu}_{2} \mathrm{~L}^{5}\left(\mathrm{NO}_{3}\right)_{2}\right]^{+}$, respectively.

The dissociation of the tetranuclear species is very important for the subsequent catalytic reactions studied with this system, in particular for the oxidative C-C coupling of 4ethylphenol, where dicopper(II) species are shown to act as the catalyst (see section 9).

\subsection{Comparison of the spectroscopic and structural features of the copper complexes}

In order to analyze and compare the geometries, both in solid state and solution, of the copper ions in the complexes described in this chapter, values for the important parameters $\tau$ and $\lambda_{\max }$ are summarized in Table 13.

Table 13. $\tau$ values and $\lambda_{\max }$ for selected copper complexes.

\begin{tabular}{cccc}
\hline Complex & $d(\mathrm{Cu} \cdots \mathrm{Cu})[\AA]$ & $\tau$ & $\lambda_{\max }[\mathrm{nm}]\left(\varepsilon\left[\mathrm{L} \mathrm{mol}^{-1} \mathrm{~cm}^{-1}\right]\right)^{*}$ \\
\hline $\mathbf{1}$ & 3.59 & $0.23 / 0.24$ & $628(282)$ \\
$\mathbf{4}$ & $\sim 3.5$ & 0.86 & $816(291)$ \\
$\mathbf{5 a}$ & 4.34 & 0.88 & $730 \mathrm{sh}(135) 962(269)$ \\
$\mathbf{6}$ & 3.87 & 0.91 & $689(281), 923(272)$ \\
$\mathbf{7}$ & 6.76 & 0.13 & $677(96)$ \\
$\mathbf{8}$ & $3.48 / 4.60$ & $0.83 / 0.95$ & $995(280), 726 \mathrm{sh}(135)$
\end{tabular}

*UV/vis spectra in solution (see experimental part)

In the crystal structure of 5a, two copper ions were found to be in almost perfect trigonal bipyramidal $\left\{\mathrm{N}_{4} \mathrm{O}\right\}$ coordination environment, and this is reflected nicely in the $\mathrm{UV} / \mathrm{vis}$ spectrum, with an absorption maximum at $962 \mathrm{~nm}$. In case of a slightly distorted square pyramidal geometry of the copper ions, as in complex $1, \lambda_{\max }$ is typically found at lower value, and the observations prove the accordance between the solid state and solution 
structure for this species. The presence of two geometrically different copper ions in complex 6 should give rise to two bands in the UV/vis spectrum for both square pyramidal and trigonal bipyramidal copper, and this was indeed confirmed. Moreover, equilibrium between dinuclear and tetranuclear species was occurred in some cases.

From the observed correlations between significant structural parameters of both the solid state and solution structure, it can be safely implied that the structures, as determined by Xray crystallography for isolated crystalline forms of these complexes, are clearly retained when in solution. This not only leads to the full characterization of these species, but more importantly shows the high structural stability of these pyrazolate-based ligand systems and their $\mathrm{Cu}$-complexes. This in turn might allow the use of solution spectroscopic techniques as elegant and credible tools for the elucidation of e.g. the characterization of specific intermediates when these $\mathrm{Cu}$-species are employed as catalysts. 


\section{Bioinspired oxidation reactions of phenols}

Unprecedented and highly interesting catalytic activity of the pyrazolate-based dicopper(II) complex with binding compartments composed of imidazolyl groups (complex 5a) in phenol oxidation will be discussed in detail. Substrate binding to the dicopper site was investigated by spectroscopic techniques and solid-state X-ray crystallography, using phenols that are inert to oxidation. A subtle interplay of reaction conditions and substrate specific properties enabled the determination of different kinds of organic products after catalysis. Mechanistic insight in the studied oxidation reactions is provided from a combination of spectroscopic studies.

\subsection{Screening of the phenol substrates}

In order to test the reactivity of the pyrazolate-based dicopper(II) complex $\mathbf{5 a}$ as a catalyst in bioinspired phenol oxidation, a screening of a range of phenol substrates was performed. It was envisioned that these very different phenols might show dissimilar tendencies to undergo chemical transformations during catalysis (Figure 40).<smiles>CC(C)(C)c1ccc(O)cc1</smiles><smiles>CC(C)(C)c1ccc(O)cc1</smiles>

tBuP<smiles>Cc1cccc(C)c1O</smiles>

DMP

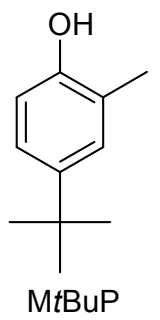<smiles>Cc1cc(C)c(O)c(C)c1</smiles>

TMP<smiles>Cc1ccc(C)c(O)c1C</smiles>

2,5,6-TMP<smiles>Cc1cc(C)c(C)c(O)c1</smiles>

2,3,5-TMP<smiles>Cc1cc(Br)cc(C)c1O</smiles>

DMBrP<smiles>CCc1ccc(O)cc1</smiles>

4-EtP



PFP<smiles>NC(=O)c1ccc(O)cc1</smiles>

4-AP

Figure 40. The set of the phenols screened for activity with dicopper complex $\mathbf{5 a}$.

The nature of the substituents on the phenol ring has a large influence on the reactivity observed with the chosen dinuclear $\mathrm{Cu}$-complex $\mathbf{5 a}$, leading to a wide variety of products and 
unexpected modes of activation of the phenolic substrates. Thus, electron rich DMP and TMP are shown to easily undergo $\mathrm{C}-\mathrm{C}$ coupling in the respective para-positions, catalyzed by dicopper(II) complex 5a (see section 6.2 and 6.3). 4-Ethylphenol, a phenol with unsubstituted ortho-positions, is found to be a particularly reactive substrate for $\mathrm{C}-\mathrm{C}$ oxidative coupling in the ortho-position (see section 9). Presence of the extremely reactive bromo substituent in the para-position of 4-bromo-2,6-dimethylphenol (DMBrP) directs the catalysis towards oxidative C-O coupling, with formation of a polymeric material, analogous to PPE (see section 11). Phenols with strong electron-withdrawing properties, such as pentafluorophenol (PFP) and 4-hydroxybenzamide (4-AP), are inert to any kind of Cu-catalyzed oxidation reaction and are therefore applied as model substrates to elucidate phenol binding to the dicopper active core (see section 8.2). Possible correlations between the observed activities of the screened phenols and their electronic and steric properties will be discussed in detail within the following chapters of the present work.

\subsection{Oxidative C-C coupling of 2,6-dimethylphenol}

2,6-dimethylphenol (DMP) is known to be oxidized in the presence of a copper-catalyst to yield the C-O coupled polymeric product poly(2,6-dimethylphenylene ether) (PPE). Therefore, to test the catalytic activity of the newly prepared dicopper complex 5a, featuring pendant imidazolyl side arms, DMP was chosen as the initial substrate. In marked contrast to previous results obtained with pyrazolate-based dicopper(II) complexes having aliphatic side arms (see section 5.2.2), whereby DMP was selectively converted to yield PPE, complex 5a was found to catalyze the oxidation of DMP in a very different manner. Thus, in the presence of 5a, DMP underwent C-C oxidative coupling, yielding 3,3',5,5'-tetramethyl-4,4'diphenoquinone (DPQ) as the main product (Scheme 29), with no traces of C-O coupled polymer found. Notably, DPQ is normally observed as by-product in the synthesis of PPE, but few selective processes have so far been devised to form DPQ as the main product from copper-catalyzed oxidative coupling of DMP. ${ }^{126}$ 
2<smiles>Cc1cccc(C)c1O</smiles>

DMP

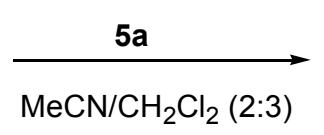

$\mathrm{MeCN} / \mathrm{CH}_{2} \mathrm{Cl}_{2}(2: 3)$

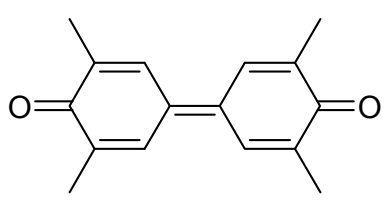

$\mathrm{DPQ}$

Scheme 29. Oxidative coupling of DMP catalyzed by dicopper complex $\mathbf{5 a}$.

Using catalyst 5a instead of the previously employed mononuclear copper systems ${ }^{88,89}$ allowed the $\mathrm{C}-\mathrm{C}$ coupling to be performed under mild conditions, avoiding high temperatures and long reaction times. ${ }^{126}$ The standard conditions used for the $\mathrm{C}-\mathrm{C}$ coupling as well as the $\mathrm{C}-\mathrm{O}$ coupling reactions of phenols require the use of additional base (e.g. triethylamine) to activate the phenolic substrate and enable interaction with the Cu-catalyst, whereas 5a already contains a highly basic methanolate that can act as an internal base. The catalytic reaction of DMP with aerial dioxygen as oxidant and in the presence of 5a takes place without addition of base and at room temperature.

The C-C coupling reaction of DMP to DPQ was monitored using time-resolved stopped-flow UV/vis spectroscopy (Figure 41).

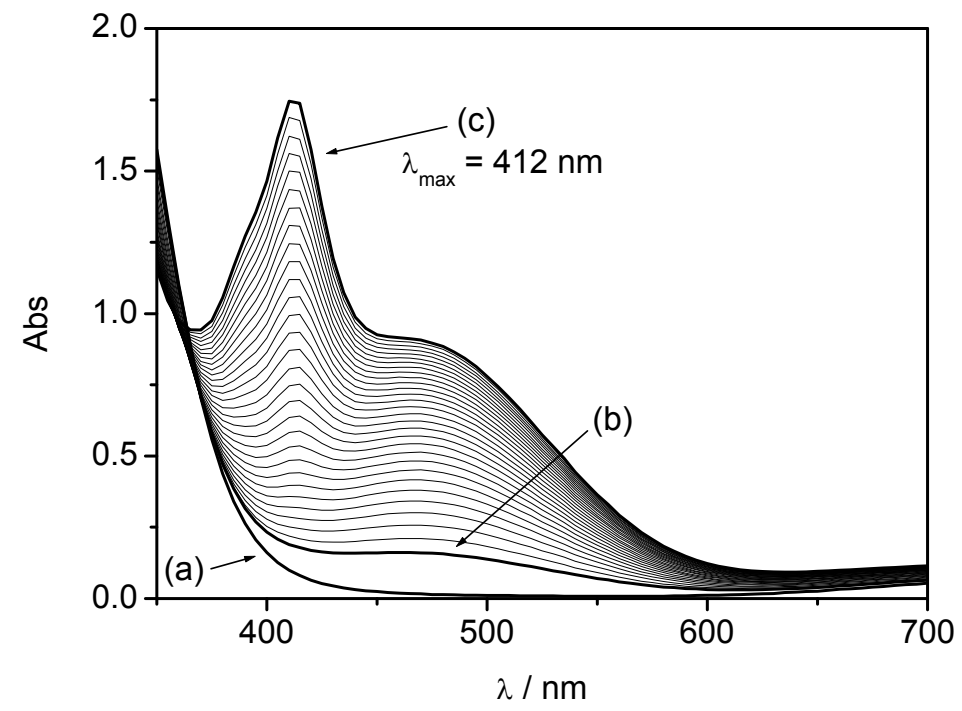

Figure 41. UV/vis spectra at different stages of the oxidative coupling of DMP (initial concentration of $\mathbf{5 a}=4 \cdot 10^{-4} \mathrm{~mol} \cdot \mathrm{L}^{-1}$; initial concentration of DMP $=2 \cdot 10^{-3} \mathrm{~mol} \cdot \mathrm{L}^{-1} ; \mathrm{L}=1.0$ cm; solvent: MeCN; (a) starting solution of 5a; (b) a few seconds after addition of TMP (red solution); (c) 6 minutes after addition of DMP (absorption spectrum of the formed DPQ as indicated by the intense absorption band at $412 \mathrm{~nm}$ ). 
Measurements were performed in the range from 350 to $900 \mathrm{~nm}$, as no $d$ - $d$ bands from the dicopper complex 5a are to be expected in this region. Drastic changes were already observed a few seconds after addition of DMP, with the appearance of an intense band at $\lambda_{\max } 472 \mathrm{~nm}$ $\left(\varepsilon \sim 1500 \mathrm{~L} \cdot \mathrm{mol}^{-1} \cdot \mathrm{cm}^{-1}\right)$. DPQ started to form after 3 minutes of reaction, as indicated by the intense absorption band at $412 \mathrm{~nm}$ (Figure 41 (c)).

This $4 \mathrm{e}^{-}$oxidation of DMP to DPQ in the presence of aerial dioxygen can not be performed in one step, as only $2 \mathrm{e}^{-}$can be taken up by the initial complex $\mathbf{5 a}$ (assuming a $\mathrm{Cu}^{\mathrm{II}} \mathrm{Cu}^{\mathrm{II}}$ to $\mathrm{Cu}^{\mathrm{I}} \mathrm{Cu}^{\mathrm{I}}$ redox-process). The overall reaction therefore likely proceeds via formation of an intermediate organic product. In accord with this hypothesis, it was observed that under anaerobic conditions the oxidative coupling reaction was halted after formation of the 3,3',5,5' -tetramethylbiphenyl-4,4'-diol (TMBD) (Scheme 30).

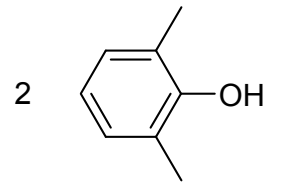

DMP

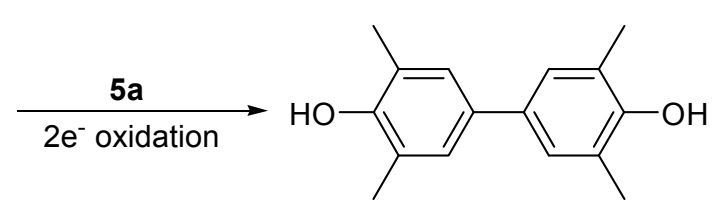

TMBD

Scheme 30. 2e- oxidative C-C coupling of DMP under anaerobic conditions.

This $2 \mathrm{e}^{-}$oxidative C-C coupling of DMP (1 $\mathrm{e}^{-}$per DMP molecule) under anaerobic conditions can only take place in parallel with reduction of the initial dicopper complex 5a. In order to elucidate the above-mentioned aspects of this $\mathrm{C}-\mathrm{C}$ coupling reaction, it was deemed logical to switch to the related compound 2,4,6-trimethylphenol (TMP) and investigate if substitution of the reactive para-position would alter or possibly even totally block the reactivity of 5a. This would then allow a study of the coordination of 'unreactive' phenols to the dicopper core of $\mathbf{5 a}$ and provide insights to elucidate some factors guiding the activity of $\mathbf{5 a}$.

\subsection{Oxidative C-C coupling of 2,4,6-trimethylphenol}

The copper-mediated oxidation of 2,4,6-trimethylphenol (TMP) with oxygen as an oxidant has been dominated by the formation of known oxygenated products such as 4-hydroxy-3,5dimethylbenzaldehyde, 2,6-dimethyl-p-benzoquinone and 4-alkoxy-2,6-dimethylphenols. ${ }^{96 \mathrm{~b}, \mathrm{c}}$ As a consequence, the oxidative coupling of TMP at the benzylic carbon in the para-position 
is rarely observed and has so far required the use of excess strong oxidants to proceed. ${ }^{127}$ The only reported incident of selective oxidative C-C coupling of TMP to yield stilbenequinone was achieved in the presence of dioxygen and a mononuclear copper complex, as published by Tsushida and co-workers. ${ }^{128}$

As mentioned in the previous paragraph, despite the remarkable selectivity towards C-C coupling that was evidenced for DMP, it was hypothesized that with TMP as substrate, such reactivity would not be observed, as the para-position is blocked for direct aryl-aryl C-C coupling.

The same reaction conditions as used for the copper-catalyzed oxidative coupling of DMP were applied, where TMP was added to a solution of 5a in a mixture of $\mathrm{MeCN} / \mathrm{CH}_{2} \mathrm{Cl}_{2}$. Red crystals, which separated from the reaction mixture after standing overnight, could be isolated and fully characterized by a combination of X-ray crystallography and spectroscopic methods. It was thereby revealed that the product obtained corresponded to 3,3',5,5'tetramethylstilbene-4,4'-quinone (TMSQ) (Scheme 31).

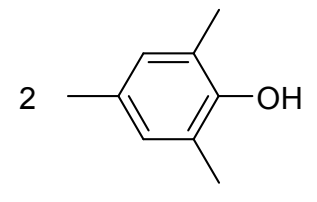

TMP

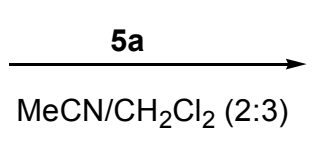

$\mathrm{MeCN} / \mathrm{CH}_{2} \mathrm{Cl}_{2}(2: 3)$

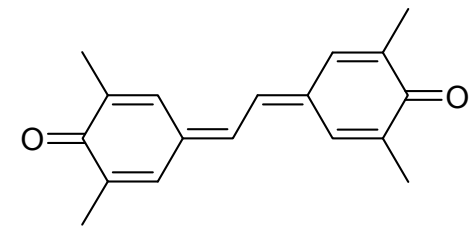

TMSQ

Scheme 31. Oxidative C-C coupling of TMP catalyzed by $\mathbf{5 a}$.

The high-yield formation of TMSQ under the applied reaction conditions established that dicopper complex 5a, incorporating functional imidazolyl side arms, shows remarkable and significant selectivity towards the oxidative C-C coupling with both DMP and TMP as substrates.

The reaction was followed in time by means of UV/vis spectroscopy. The electronic spectrum of $5 \mathbf{a}$ in $\mathrm{MeCN} / \mathrm{CH}_{2} \mathrm{Cl}_{2}$ showed a band at $\lambda_{\max } 989 \mathrm{~nm}\left(\varepsilon=269 \mathrm{~L} \cdot \mathrm{mol}^{-1} \cdot \mathrm{cm}^{-1}\right)$ with a shoulder at $724 \mathrm{~nm}$, corresponding to $d$ - $d$ transitions of copper(II) ions in a trigonal bipyramidal environment (Figure 42 (a)). Significant changes were observed directly after addition of TMP (Figure 42 (b)), namely the appearance of an intense band at $\lambda_{\max } 495 \mathrm{~nm}(\varepsilon \sim 1500$ 
$\mathrm{L} \cdot \mathrm{mol}^{-1} \cdot \mathrm{cm}^{-1}$ ) and a shift of the $\mathrm{Cu}^{\mathrm{II}}$-based $d-d$ transition band to $960 \mathrm{~nm}$. Formation of the product (TMSQ) began after a relatively long incubation period $(2 \mathrm{~h})$ and the intense absorption band of the product is detected at $440 \mathrm{~nm}\left(\varepsilon \sim 96000 \mathrm{~L} \cdot \mathrm{mol}^{-1} \cdot \mathrm{cm}^{-1}\right.$ in $\left.\mathrm{CH}_{2} \mathrm{Cl}_{2}\right)$ (Figure $42(\mathrm{c})$ ).

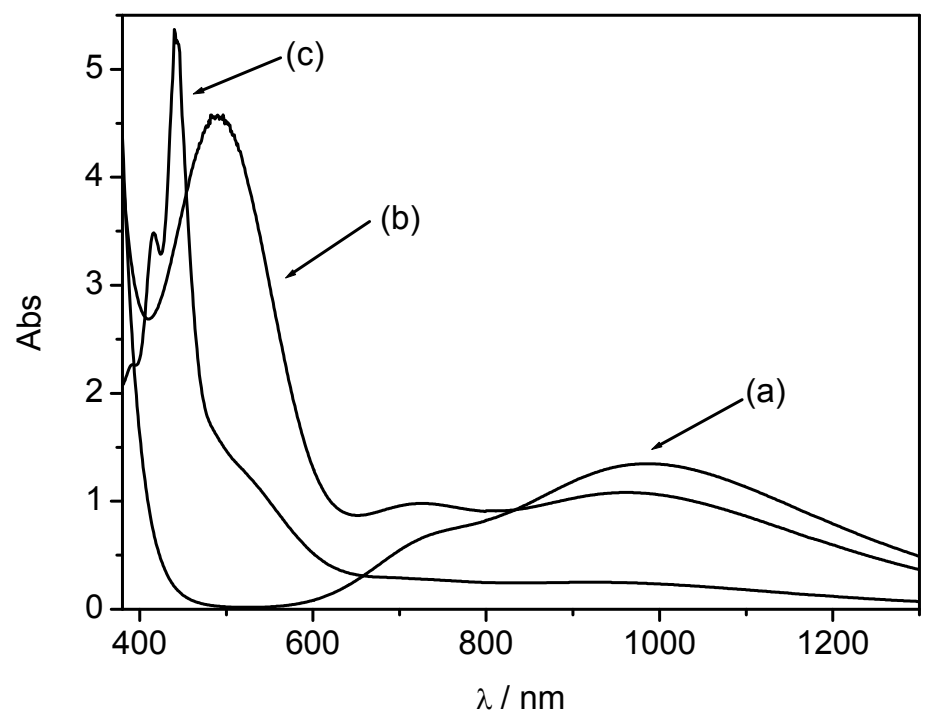

Figure 42. UV/vis spectra at different stages of the oxidative coupling of TMP (initial concentration of $\mathbf{5 a}=0.005 \mathrm{~mol} \cdot \mathrm{L}^{-1}$; initial concentration of $\mathrm{TMP}=0.025 \mathrm{~mol} \cdot \mathrm{L}^{-1} ; \mathrm{L}=1.0$ cm; solvent: $\mathrm{MeCN} / \mathrm{CH}_{2} \mathrm{Cl}_{2}$ 4:6 v/v): (a) starting solution of 5a; (b) a few minutes after addition of TMP (red solution); (c) $8 \mathrm{~h}$ after addition of TMP (absorption spectrum of the formed TMSQ scaled by a factor 90$)$.

One notable difference with the related reactivity observed for DMP with complex 5a is that the oxidative C-C coupling of TMP to yield TMSQ is an overall 6e-process $\left(3 \mathrm{e}^{-}\right.$per TMP molecule), which implies a rather complicated reaction pathway and hence intriguing mechanistic considerations. Further studies on the mechanism of C-C coupling of TMP will be discussed in details (see section 7). 


\section{Investigations of the mechanism of C-C coupling of TMP}

\subsection{Determination of the optimal reaction conditions for C-C-coupling of TMP}

Optimization of the reaction conditions was first of all focussed on improving the yield of TMSQ, which was targeted by studying the solvent influence on the catalytic performance. The yield of TMSQ was determined by means of UV/vis spectroscopy, with the aid of the intense absorption band of this product at $440 \mathrm{~nm}\left(\varepsilon \sim 96000 \mathrm{~L} \cdot \mathrm{mol}^{-1} \cdot \mathrm{cm}^{-1}\right)$. It was found that reactions performed in a 2:3 mixture of $\mathrm{MeCN} / \mathrm{CH}_{2} \mathrm{Cl}_{2}$ provided the highest yield of TMSQ, approximately $65 \%$. Further studies were concentrated on the influence of the substrate (TMP) to catalyst 5a (S/C) ratio on the yield of TMSQ. Six identical samples of the dicopper(II) complex 5a in $\mathrm{MeCN} / \mathrm{CH}_{2} \mathrm{Cl}_{2}$ (2:3) with identical concentration (0.0024 M) were prepared and TMP was added as a solid to each solution, but with varying initial concentration $(0.0026 \mathrm{M}, 0.0050 \mathrm{M}, 0.0131 \mathrm{M}, 0.0260 \mathrm{M}, 0.0480 \mathrm{M}, 0.0655 \mathrm{M})$. The red coloured mixtures were stirred for $24 \mathrm{~h}$ at room temperature and after appropriate dilution of the reaction samples with $\mathrm{CH}_{2} \mathrm{Cl}_{2}$, UV/vis spectra were recorded. The experimental data showed that the yield in TMSQ was only slightly dependent on the ratio TMP:5a, with the most optimal S/C ratio at 5:1 (TMP $(0.0131 \mathrm{M}): 5 a(0.0024 \mathrm{M}))$, giving $66 \%$ of TMSQ (Figure 43).

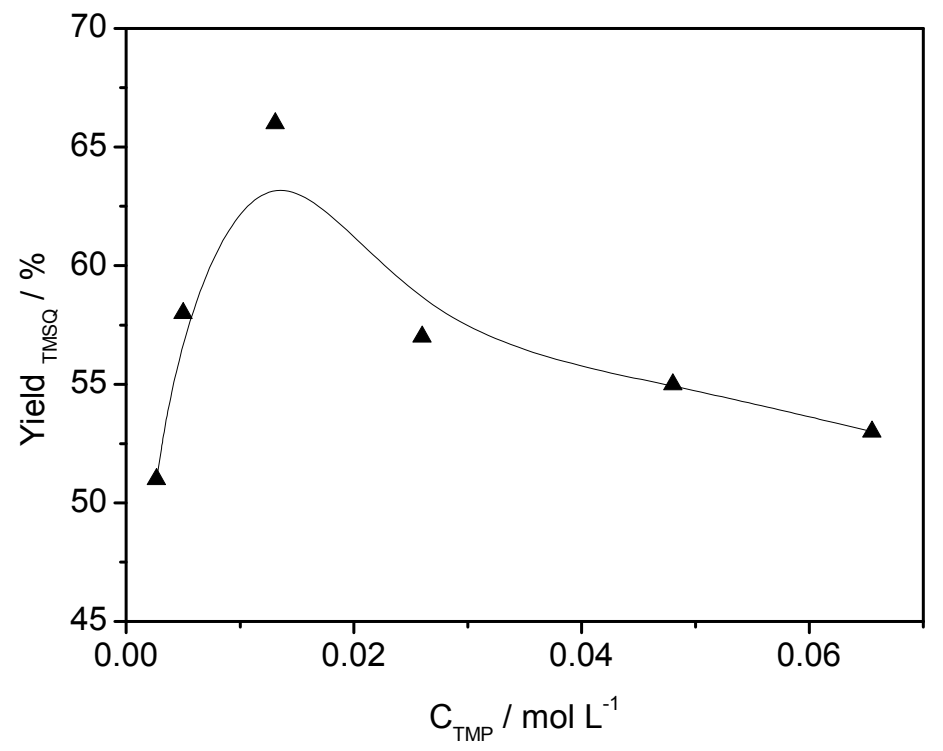

Figure 43. Dependence of the yield of TMSQ on the S/C ratio, as followed by UV/vis spectroscopy, with a constant concentration in catalyst 5a $(0.0024 \mathrm{M})$. 
The TON number (expressed as mole substrate converted per mole catalyst used) increased linearly with the concentration of TMP employed in the reaction, which is a clear indication for high catalyst stability and good overall performance of the catalytic active dicopper complex (Figure 44).

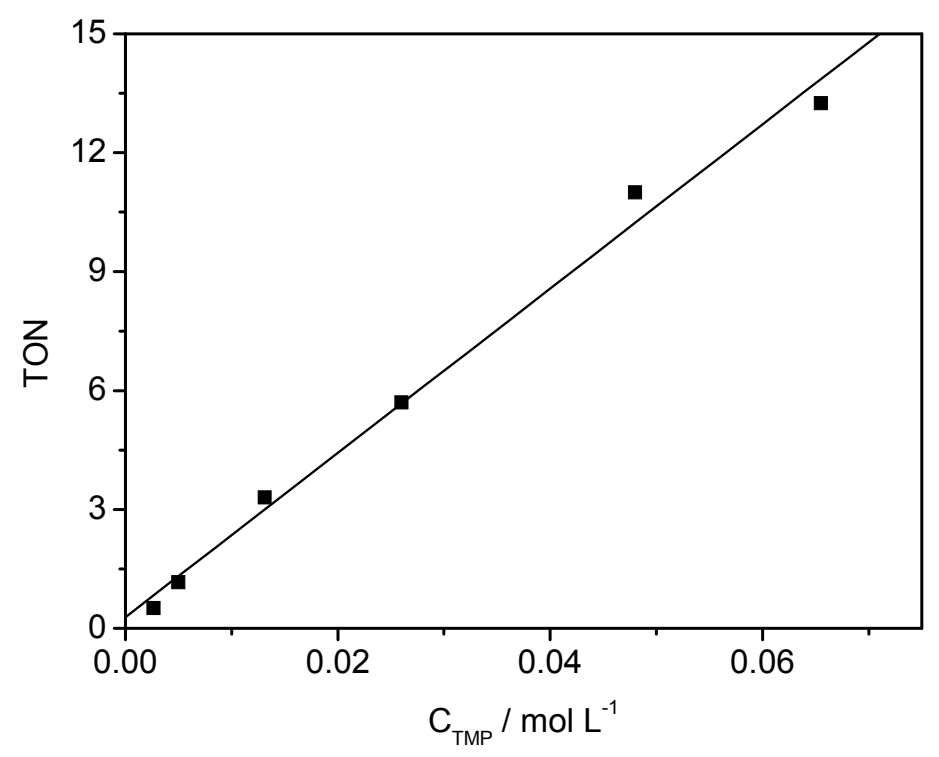

Figure 44. Turnover numbers for formation of TMSQ (after $24 \mathrm{~h}$ for each sample).

Thus, as the catalytic activity of 5a was determined to not be strongly dependent on the substrate to catalyst ratio, S/C 5:1 ratio was chosen as standard condition for further studies. The temperature profile of the yield in TMSQ was not investigated, because it was reasoned that the oxidative C-C coupling of TMP with copper catalyst 5a could attract the greatest interest when selectively conducted under very mild conditions, i.e. at room temperature and in the absence of excess base or other additives.

\subsection{Investigations with deuterated TMP substrate}

It is known that 2,6-dimethylphenol (DMP) can be oxidized in the presence of a coppercatalyst, to yield the $\mathrm{C}-\mathrm{O}$ coupled polymeric product poly(2,6-dimethylphenylene ether) (PPE) as well as the C-C coupled product 3,3',5,5'-tetramethyl-4,4'-diphenoquinone (DPQ). Many studies were done in order to investigate the mechanism of the oxidative polymerization of DMP (see section 3.2) and it was shown that the reaction can proceed through free radical intermediates and, moreover, that migration of the $\mathrm{H}$-atom from the para- 
position to the meta-position of the phenol is taking place during the polymerization process (Scheme 32). ${ }^{129}$

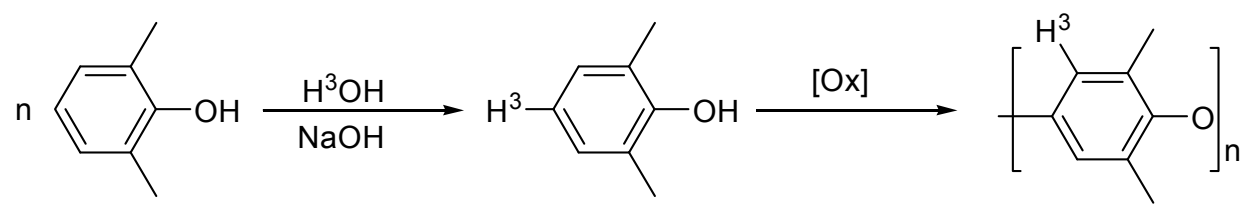

Scheme 32. H-atom migration during the C-O coupling of DMP.

With the aim to further elucidate the mechanism of the oxidative C-C coupling of TMP, such an H-atom migration pathway had to be considered as a possible step in the mechanism. Therefore, NMR spectroscopy was used to clarify whether this was indeed a viable option to take into account for the overall mechanistic picture for this reaction.

First of all, the substrate with fully deuterated $\mathrm{CH}_{3}$-groups $\left(C D_{3}\right.$-TMP) was prepared (via standard Raney-Ni catalyzed H/D exchange for TMP, using $\mathrm{D}_{2} \mathrm{O}$ as the deuterium source $)^{130}$ and tested in the $\mathrm{C}-\mathrm{C}$ coupling. Under the same reaction conditions as for the $\mathrm{C}-\mathrm{C}$ coupling of TMP, $C D_{3}$-TMP smoothly underwent reaction to yield $\mathrm{TM}^{\mathrm{D}} \mathrm{SQ}-$ the analogue of TMSQ with deuterated $\mathrm{CH}_{3}$-groups (Scheme 33).

2<smiles>[R]Oc1cc(C([2H])([2H])[2H])c(O)c(C([2H])([2H])[2H])c1</smiles>

$C D_{3}-\mathrm{TMP}$

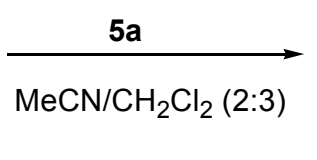

$$
\mathrm{D}_{3}
$$

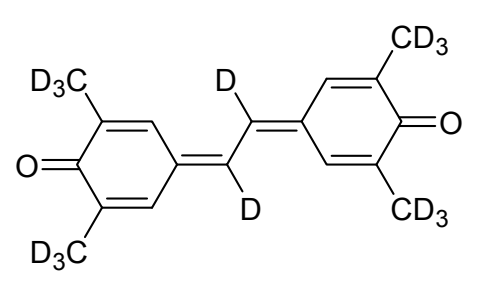

$T M^{\mathrm{D}} \mathrm{SQ}$

Scheme 33. Oxidative C-C coupling of $C D_{3}$-TMP catalyzed by $\mathbf{5 a}$.

The formed product $T M^{\mathrm{D}} \mathrm{SQ}$ was straightforwardly characterized by standard ${ }^{1} \mathrm{H}$ and ${ }^{13} \mathrm{C}$ NMR spectroscopy, but the obtained results did not allow any unambiguous distinction between products formed with or without possible H-atom migration (Figure 45). 


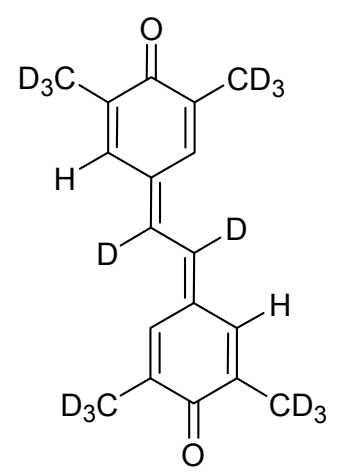

a

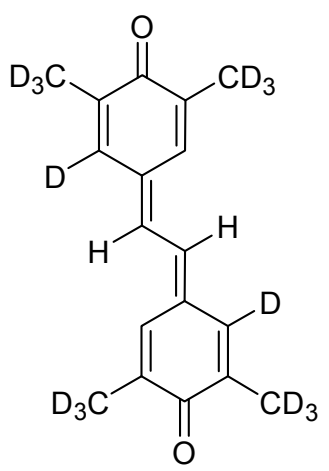

b

Figure 45. Two possible TM ${ }^{\mathrm{D}} \mathrm{SQ}$ products formed: (a) without H-atom migration; (b) with Hatom migration.

Further determination of the $\mathrm{TM}^{\mathrm{D}} \mathrm{SQ}$ structure was carried out using Heteronuclear Multiple Quantum Corrrelation (HMQC) and Heteronuclear Multiple Bond Correlation (HMBC) NMR techniques. Both of them are two-dimensional inverse $\mathrm{C}, \mathrm{H}$-correlation techniques that provide information about the carbon (or other heteroatoms) to hydrogen connectivity. HMQC is selective for direct $\mathrm{C}-\mathrm{H}$ coupling interactions, while HMBC will give longer range couplings (two- to four-bond coupling) between heteroatom nuclei. The 1D HMQC spectrum of $\mathrm{TM}^{\mathrm{D}} \mathrm{SQ}$ was recorded in $\mathrm{CDCl}_{3}$ (Figure 46).



Figure 46. 1D HMQC spectrum of $\mathrm{TM}^{\mathrm{D}} \mathrm{SQ}$ in $\mathrm{CDCl}_{3}$. 
Two signals for two protons from $\mathrm{TM}^{\mathrm{D}} \mathrm{SQ}$, which appear at $\delta 7.02$ and $7.50 \mathrm{ppm}$ in the ${ }^{1} \mathrm{H}$ NMR spectrum, show a mutual coupling with a coupling constant $J_{\mathrm{HH}}$ of $2.6 \mathrm{~Hz}$, as well as short range C-H coupling with $J_{\mathrm{CH}}=160 \mathrm{~Hz}$. The observed value for the $J_{\mathrm{HH}}$ coupling is typical for aromatic protons in meta-position to each other, as found in the proposed structure for $\mathrm{TM}^{\mathrm{D}} \mathrm{SQ}$ formed without $\mathrm{H}$-atom migration, i.e. structure (a). It has to be mentioned that in case H-atom migration would have occurred (structure (b)), the corresponding 1D HMQC spectrum should include signals corresponding to a $\mathrm{H}, \mathrm{H}$ trans coupling with a value for the coupling constant $J_{\mathrm{HH}}$ of $\sim 7-10 \mathrm{ppm}$.

The HMBC method, which is selective for longer range couplings (two- to four-bond coupling), was applied to obtain additional evidence about the actual structure of $\mathrm{TM}^{\mathrm{D}} \mathrm{SQ}$ and to support the preliminary conclusion, that no $\mathrm{H}$-atom migration takes place during its formation. In the recorded $\mathrm{HMBC}$ spectrum both protons from $\mathrm{TM}^{\mathrm{D}} \mathrm{SQ}$ resonating at $\delta 7.02$ and $7.50 \mathrm{ppm}$ in the ${ }^{1} \mathrm{H}$ NMR spectrum, showed four bond coupling to the carbonyl C-atom $(\mathrm{C}=\mathrm{O})$ at $185 \mathrm{ppm}$, which is possible only in the case of proposed structure (a).

On the basis of these results, it can be safely concluded that the oxidative C-C coupling of TMP in the presence of 5a and dioxygen is taking place without $\mathrm{H}$-atom migration from the para $\mathrm{CH}_{3}$-group to the meta-position of the aromatic ring.

\subsection{Discussion of the nature of the UV/vis Charge Transfer band of dicopper phenolate complexes}

Addition of DMP and TMP substrates to a green solution of dicopper(II) complex 5a in $\mathrm{MeCN}$ led to a colour change of the solution from green to red with concomitant appearance of an intense band at $\lambda_{\max }=472$ and 495 , respectively. These spectral changes are most likely occurred do to the coordination of a phenolate to one of the copper(II) ions of complex 5a owing to a methanolate-phenolate exchange. Appearance of such an intense band in UV/vis spectrum upon addition of phenols to various copper complexes was observed in similar systems. $^{131,132,133}$ and, moreover, this band was characterized as a ligand-to metal chargetransfer band (LMCT).

In order to determine the nature of the observed charge-transfer band, addition of the various phenol substrates to a green solution of 5a was performed. Identical colour change, as for 
DMP and TMP was observed, with concomitant appearance of an intense band with $\lambda_{\max }$ in the range from 390 to $500 \mathrm{~nm}$ (Table 14).

Table 14. Various phenols used for complexation studies with dicopper complex $5 \mathbf{a}$ and $\lambda_{\max }$ of the CT band of the corresponding phenolate adducts.

\begin{tabular}{c|c}
\hline phenol & $\lambda_{\max }(\mathrm{nm})$ \\
\hline $4-\mathrm{MeP}$ & 457 \\
DMP & 472 \\
TMP & 495 \\
$t \mathrm{BuP}$ & 455 \\
$4-\mathrm{AP}$ & 440 \\
PFP & 397 \\
\hline
\end{tabular}

A clear trend was observed in $\lambda_{\max }$ for the different phenols used, in particular for the three methyl-substituted phenols 4-MeP, DMP and TMP. The energy of the charge-transfer transition of these copper-phenolate complexes will decrease with an increase in the ability of the coordinated phenol to donate electron density (originating from the $\mathrm{CH}_{3}$-groups on the phenol) to one of the copper ions, resulting in a shift of the charge-transfer (CT) band in the $\mathrm{UV} / \mathrm{vis}$ spectrum to higher wavelength, i.e. lower energy. Therefore, the charge-transfer band in these particular cases was identified as a ligand-to-metal (LMCT) band, from the phenolate to the copper(II) ion. The presence of strong electron-withdrawing groups (e.g. F or $\mathrm{CONH}_{2}$ ) on the phenol induces a rather big shift of the CT band to lower wavelength, as clearly seen in the UV/vis spectrum of the copper pentafluorophenol adduct ( $\lambda_{\max } 397 \mathrm{~nm}$ ). Previous studies on the determination of the nature of charge-transfer band for mononuclear copper-phenolate complexes with simple amine ligands (e.g. pyridine) using substituted chlorophenols have shown that the presence of strong electron-withdrawing groups typifies the CT band as a metal-to-ligand charge transfer. ${ }^{131}$ Therefore the CT band of the copper pentafluorophenolate adduct may possibly be better qualified as a metal-to-ligand charge transfer (MLCT) band. 


\subsection{Determination of the nature of the adduct formed upon the addition of TMP}

Oxidative C-C coupling of phenols, in particular DMP, as catalyzed by copper complexes, including dicopper species 5a, can be envisioned to occur via three different mechanistic pathways depicted in Scheme 34.

(a) Radical<smiles>CC1=CC([CH2+]c2cc(C)c(Oc3c(C)cc(C4C=C(C)C(=O)C(C)=C4)cc3C)c(C)c2)C=C(C)C1=O</smiles>

(b) Electrophilic<smiles>CC1=CC([CH2+]c2cc(C)c(Oc3ccccc3)c(C)c2)=CC(C)C1=O</smiles><smiles></smiles>

(c) Nucleophilic<smiles>[CH+]C1C=C(C)C(=O)C(C)=C1</smiles><smiles>CC1=CC(C2C=C(C)C(=O)C(C)=C2)C=C(C)C1=O</smiles>

Scheme 34. Mechanistic pathways proposed for the oxidative C-C coupling of DMP. ${ }^{134}$

Privious studies have shown that treatment of DMP with a radical initiator like benzoyl peroxide produced the 2,6-dimethyl-2,5-cyclohexadienone radical ${ }^{135,136}$, which will mainly undergo C-C coupling yielding DPQ as the product. On these grounds, the formation of DPQ is believed to be a radical process, where DMP coordinates to a copper(II) ion with further formation of a copper(I)-phenoxyl radical adduct.

In this sense, the unusual C-C coupling of TMP catalyzed by dicopper(II) complex 5a can in principle also occur via the three mentioned mechanistic pathways. In order to elucidate and determine the most plausible mechanistic route, Raman and EPR spectroscopy were applied as useful instruments to investigate the phenol binding within the dicopper pocket, with a further possibility to identify the reactive species formed upon substrate coordination, 
particularly distinguishing between copper(II)-phenolate and copper(I)-phenoxyl radical character.

\subsubsection{Raman spectroscopy}

Coordination of the TMP substrate to the dicopper core of 5a during the oxidative C-C coupling reaction was proven by means of UV/vis spectroscopy, particularly through the observation of an intence band with $\lambda_{\max }$ of $495 \mathrm{~nm}\left(\varepsilon \sim 1500 \mathrm{~L} \mathrm{~mol}^{-1} \mathrm{~cm}^{-1}\right)$. This band was assigned to a phenolate-to-copper LMCT transition, based on the relative shift in the UV/vis spectrum depending on the substituents in the phenol ring (see section 7.3). These studies are in good agreement with reported literature data for various TACN-supported mono- and bis(phenolate) copper(II) complexes, with $\lambda_{\max }$ in the range of $\sim 430-540 \mathrm{~nm}(\varepsilon \sim 1000-2000$ $\left.\mathrm{L} \mathrm{mol}^{-1} \mathrm{~cm}^{-1}\right) .{ }^{137,138,139}$ In marked contrast to the spectroscopic features observed for such copper-phenolate complexes, the UV/vis absorption spectra of the $\mathrm{Cu}^{\mathrm{II}}$-phenoxyl radical complexes show an intense CT band at $\lambda_{\max } \sim 390-430 \mathrm{~nm}\left(\varepsilon \sim 3000-8000 \mathrm{~L} \mathrm{~mol}^{-1} \mathrm{~cm}^{-1}\right)$ that is generally assigned to the phenoxyl $\pi-\pi^{*}$ transition on the basis of enhancements of radical ring Raman modes upon laser excitation (vide infra). ${ }^{138,139,140}$ This initial spectroscopic observation already strongly suggests that coordination of a molecule of TMP within the bimetallic pocket of 5a leads to the formation of the copper(II)-phenolate and not to a copper(I)-phenoxyl radical complex. It should be noted, however, that spectral characteristics of a copper(I)-phenoxyl radical might be somewhat different from those of a copper(II)phenoxyl radicals.

Resonance Raman spectroscopy is particularly useful for gaining insight into the structural and electronic properties of both metal-phenoxyl radical as well as metal-phenolate species, and moreover, to distinguish between them. ${ }^{138,139,140,141,142,143,144}$ Laser excitation into the $\pi$ $\pi^{*} \mathrm{UV} / \mathrm{vis}$ band $\left(\lambda_{\max } \sim 400 \mathrm{~nm}\right.$ ) of TACN-supported mono- and bis(phenoxyl radical) copper(II) and zinc(II) complexes - prepared by chemical or electrochemical oxidation of those copper-phenolate compounds - resulted in resonance Raman spectra very similar to those of free phenoxyl radicals, ${ }^{145,146}$ with two dominant features at $\sim 1500 \mathrm{~cm}^{-1}\left(v_{7 \mathrm{a}}\right.$, predominantly C-O stretching) and $\sim 1600 \mathrm{~cm}^{-1}$ ( $v_{8 \mathrm{a}}, \mathrm{C}_{\text {ortho }}-\mathrm{C}_{\text {meta }}$ ring stretching). The $v_{7 \mathrm{a}}$ band in these complexes occurs at $\sim 250 \mathrm{~cm}^{-1}$ higher energy than in the metal-phenolate precursors, indicative of increased C-O double bond character in the coordinated phenoxyl radicals. 
The Raman spectra of the copper(II)-phenolate complexes generally show a set of multiple features between 1100 and $1600 \mathrm{~cm}^{-1}$ that are typical for metal-tyrosinase sites in proteins at $1174,1270,1500$, and $1598 \mathrm{~cm}^{-1}$ (see also section 8.1).

Therefore, Resonance Raman spectroscopy was used in order to provide additional evidence for the formation of either the copper(II)-phenolate or the copper(I)-phenoxyl radical active species upon the coordination of TMP. Because of the spectroscopic features observed in the $\mathrm{UV} / \mathrm{vis}$ spectrum, laser excitation was performed at $488 \mathrm{~nm}$, into the LMCT band of the adduct with a band at $495 \mathrm{~nm}$. TMP was added to the green solution of dicopper(II) complex 5a and the Raman spectrum of the resulting red solution was recorded (Figure 47).



Figure 47. Resonance Raman spectrum of the mixture of 5a and TMP in MeCN; $\lambda_{\text {ex }}=488$ $\mathrm{nm}$, solvent signals are subtracted.

Typical features for a phenolate coordinated to copper(II) were observed in the Raman spectrum with bands at 1156 ( $v_{9 \mathrm{a}} \mathrm{C}-\mathrm{H}$ bending), 1237 ( $v_{7 \mathrm{a}} \mathrm{C}-\mathrm{O}$ stretching), and $1607 \mathrm{~cm}^{-1}\left(v_{8 \mathrm{a}}\right.$ $\mathrm{C}_{\text {ortho }}-\mathrm{C}_{\text {meta }}$ ring stretching). The remaining three signals could not be identified, but they most probably represent the $\mathrm{C}-\mathrm{H}$ bending and $\mathrm{C}-\mathrm{C}$ stretching bands of the TMP substrate molecule. For thorough assignment of the characteristic vibrations corresponding to the coordinated phenolate-ring, in particular for the copper-phenolate adduct derived from 5a and TMP, a model $C D_{3}$-TMP substrate with fully deuterated mehyl groups was used. The modified TMP substrate is also active in the oxidative $\mathrm{C}-\mathrm{C}$ coupling in the para-position and behaves in the same fashion towards the dicopper catalyst (i.e. formation of the copper-phenolate adduct, ultimately yielding the $\mathrm{C}-\mathrm{C}$ coupled product $\mathrm{TM}^{\mathrm{D}} \mathrm{SQ}$; see section 7.2). The Raman spectrum 
of the reaction mixture of $\mathbf{5 a}$ with $C D_{3}$-TMP was recorded under the same conditions as with TMP and is depicted in Figure 48.

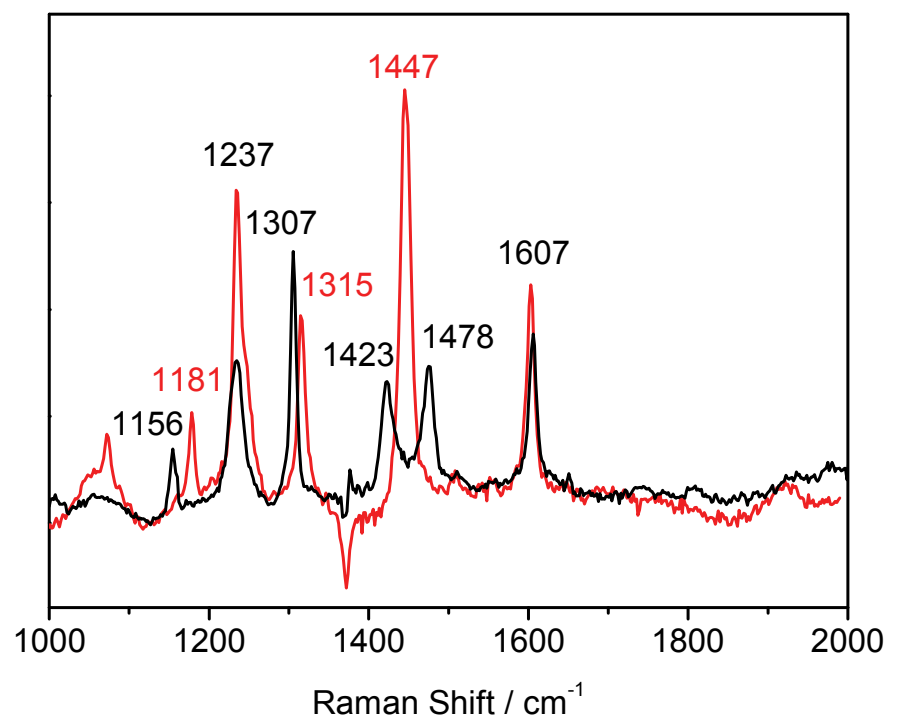

Figure 48. Resonance Raman spectra: $\lambda_{\mathrm{ex}}=488 \mathrm{~nm}$; black line - mixture of 5a and TMP in $\mathrm{MeCN}$; red line - mixture of $\mathbf{5} \mathbf{a}$ and $C D_{3}$-TMP in MeCN, solvent signals are subtracted.

Two peaks at 1237 and $1607 \mathrm{~cm}^{-1}$ in the original Raman spectrum with TMP remained unaffected by the introduction of the $C D_{3}$-groups in the phenol ring. This result is in good agreement with the definition of the stretching character of the two mentioned signals: $v_{7 \mathrm{a}}(\mathrm{C}$ $\mathrm{O}$ stretching) and $v_{8 \mathrm{a}}\left(\mathrm{C}_{\text {ortho }}-\mathrm{C}_{\text {meta }}\right.$ ring stretching $)$ which should not have any $\mathrm{C}-\mathrm{H}$ contribution. A strong influence of the $C D_{3}$-groups on the other stretching values of the phenol ring is indicated by the observed shifts of the signals at 1156 and $1307 \mathrm{~cm}^{-1}$ to higher wavenumbers $\left(\Delta v\right.$ of $+25 \mathrm{~cm}^{-1}$ and $\left.+8 \mathrm{~cm}^{-1}\right)$. Therefore, any contribution of the $\mathrm{CH}_{3}$-groups on the two mentioned bands can be excluded and, moreover, introduction of the $\mathrm{CD}_{3}$-groups influenced only the $\mathrm{C}-\mathrm{H}$ bending of the phenol ring itself. This is in good agreement with the fact that the signal at $1156 \mathrm{~cm}^{-1}$ is assigned as $\mathrm{C}-\mathrm{H}$ bending of the phenolate-ring for parasubstituted phenols. ${ }^{147}$

Moreover, the oxidative C-C coupling of TMP catalyzed by $\mathbf{5} \mathbf{a}$ to yield TMSQ was followed by Raman spectroscopy over a period of $\sim 8 \mathrm{~h}$. Formation of the quinone product had already started after $2 \mathrm{~h}$ of reaction time (Figure 49) as indicated by the appearance of signals at 1204, 1264, 1530 and $1634 \mathrm{~cm}^{-1}$ assigned to TMSQ, which most likely correspond to C-H bending (first signal), C-O and the ring C-C stretching vibrations (two last signals), respectively. 


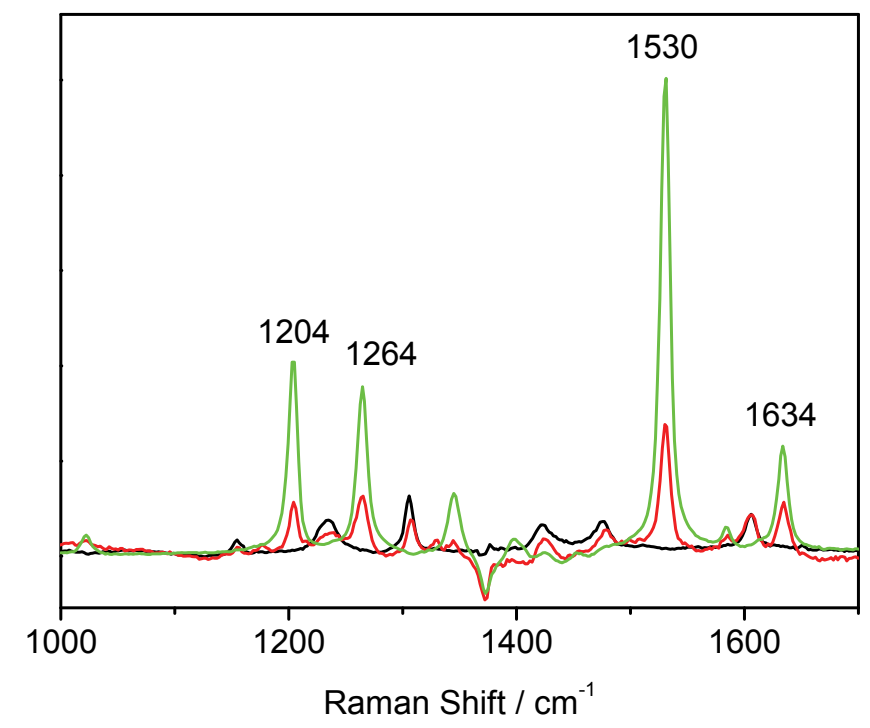

Figure 49. Resonance Raman spectra: $\lambda_{\mathrm{ex}}=488 \mathrm{~nm}$; black line - mixture of 5a and TMP in $\mathrm{MeCN}$; red line - mixture of 5a and TMP after 140 min; green line - TMSQ in MeCN, solvent signals are subtracted.

In order to learn more about the spectroscopic features of the dinuclear copper(II)-phenolate adducts with 5a, dicopper complex 5a was reacted with various phenols that are inert in any kind of oxidation reaction, focussing in particular on 2-methyl-4-tert-butylphenol ( $\mathrm{M} t \mathrm{BuP})$. Laser excitation into the LMCT band of the copper-phenolate adduct with $\mathrm{M} t \mathrm{BuP}\left(\lambda_{\max } \sim 475\right.$ $\mathrm{nm}$ ) led to the resonance effect in the Raman spectrum depicted in Figure 50.

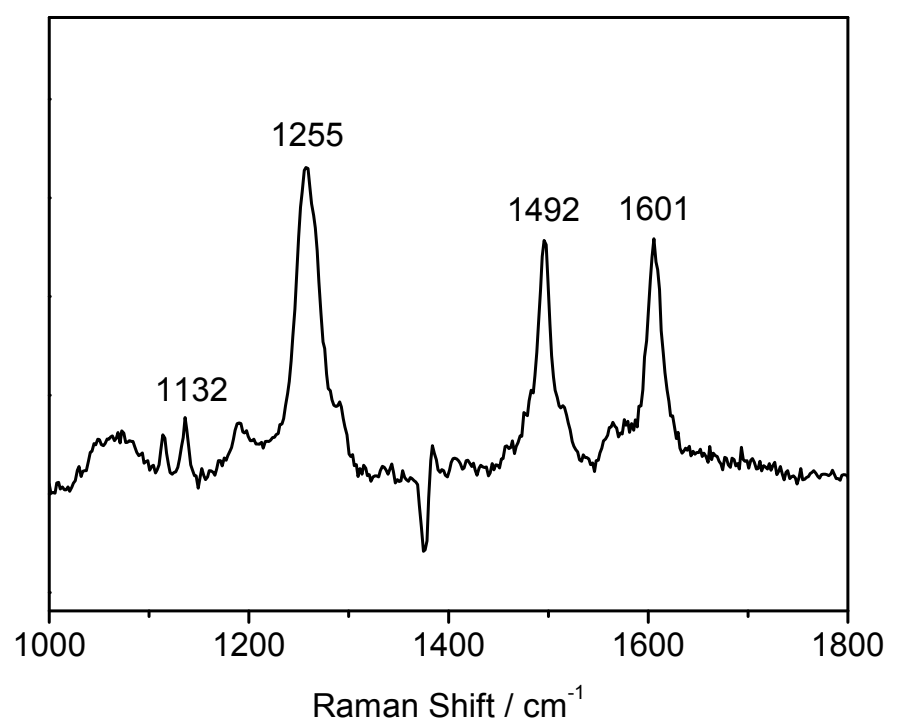

Figure 50. Resonance Raman spectrum of the mixture of 5a and $\mathrm{M} t \mathrm{BuP}$ in $\mathrm{MeCN} ; \lambda_{\mathrm{ex}}=488$ $\mathrm{nm}$, solvent signals are subtracted. 
The Raman spectrum of the copper(II)-phenolate complex formed upon addition of $\mathrm{M} t \mathrm{BuP}$ showed typical features for phenolate-ring deformation at 1132, 1255, 1492 and $1601 \mathrm{~cm}^{-1}$. Hence, the detected signals appeared in the same range as for the copper-phenolate species with the active TMP substrate and the only difference observed is the absence of the signals at 1307, 1423 and $1478 \mathrm{~cm}^{-1}$; these presumably correspond to some modified copper(II)phenolate active species formed further on in the catalytic reaction with TMP or to a TMP molecule coordinated within the bimetallic pocket itself.

The Raman measurements clearly allow the species formed upon coordination of the TMP within the bimetallic pocket of $\mathbf{5 a}$ to be described as a copper(II)-phenolate adduct.

\subsubsection{EPR studies}

The use of EPR spectroscopy is very limited in case a phenoxyl radical is coordinated to copper(II), due to the antiferromagnetic coupling between the unpaired electron of the copper(II) ion and the electron of the radical species. Therefore, in order to gain insight into the spectroscopic properties of the coordinated phenoxyl radical without interference from features due to the $d^{9} \mathrm{Cu}^{\mathrm{II}}$ ion, many studies were performed on the $\mathrm{Zn}^{\mathrm{II}}$-phenoxyl radical analogs. ${ }^{139,143,148}$ The EPR spectra of these complexes exhibit an $S=1 / 2$ signal centered at $g \sim$ 2.0, similar to those for free phenoxyl radicals. ${ }^{149}$

It was shown, by means of both UV/vis and Raman spectroscopy, that coordination of the TMP substrate to the dicopper(II) core of 5a led to formation of a dicopper(II)-phenolate species. EPR studies of the red solution containing this dicopper(II)-phenolate species at room temperature were not informative: the broad signal, observed for dicopper(II) complex 5a (which is typical for dinuclear copper(II) complexes with pyrazole-based ligands at room temperature) remained completely unchanged upon addition of TMP. Studies at lower temperature $(120 \mathrm{~K})$ revealed the same result. This broad signal with a weak half-field signal, characteristic for an exchange-coupled antiferromagnetic dicopper(II) system in the case of $\mathbf{5 a}$ appears as the result of significant interaction between the two copper(II) ions and only at very low temperature $(15 \mathrm{~K})$ this complex is EPR-silent, due to antiferromagnetic coupling (see section 5.4.4) and an $\mathrm{S}=0$ ground state (Figure 51). 


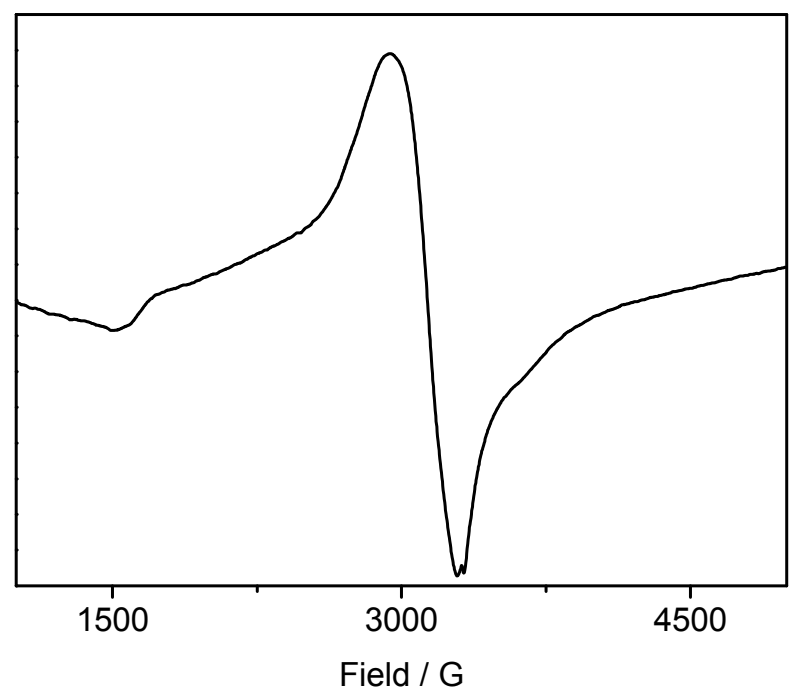

Figure 51. EPR spectrum of $\mathbf{5 a}$ in MeCN glass at $50 \mathrm{~K}$.

On the one hand it can be argued that this particular interaction between two copper(II) ions is also observed in the dicopper(II)-phenolate species, leading to the same EPR behaviour as for 5a. On the other hand, the broad signal in the EPR spectrum of the red solution can be interpreted as stemming from a copper(II)copper(I)-phenoxyl radical species, which would also yield a spectrum for an exchange-coupled system, and might not be very different from that of $\mathbf{5 a}$.

With the idea to exclude any interaction between the $d^{9}$ copper(II) ions upon coordination of the TMP molecule, a model dinuclear copper-zinc complex was synthesized to perform analogous EPR studies on a system where the second metal ion is EPR silent. To a solution of the deprotonated ligand $\mathbf{H L}^{4}$ in $\mathrm{MeCN} / \mathrm{MeOH}(1: 1, \mathrm{v} / \mathrm{v})$ one equivalent of both $\mathrm{Cu}\left(\mathrm{ClO}_{4}\right)_{2} \cdot 6 \mathrm{H}_{2} \mathrm{O}$ and $\mathrm{Zn}\left(\mathrm{ClO}_{4}\right)_{2} \cdot 6 \mathrm{H}_{2} \mathrm{O}$ were added. The obtained blue-green solution was additionally treated with one equivalent of $\mathrm{KO} t \mathrm{Bu}$ to establish a hydrogen-bonded $\mathrm{MeO} \cdots \mathrm{HOMe}$ bridge between the two metal ions. The EPR spectrum of the frozen solution of this copper-zinc complex was measured at $15 \mathrm{~K}$ (Figure 52). 


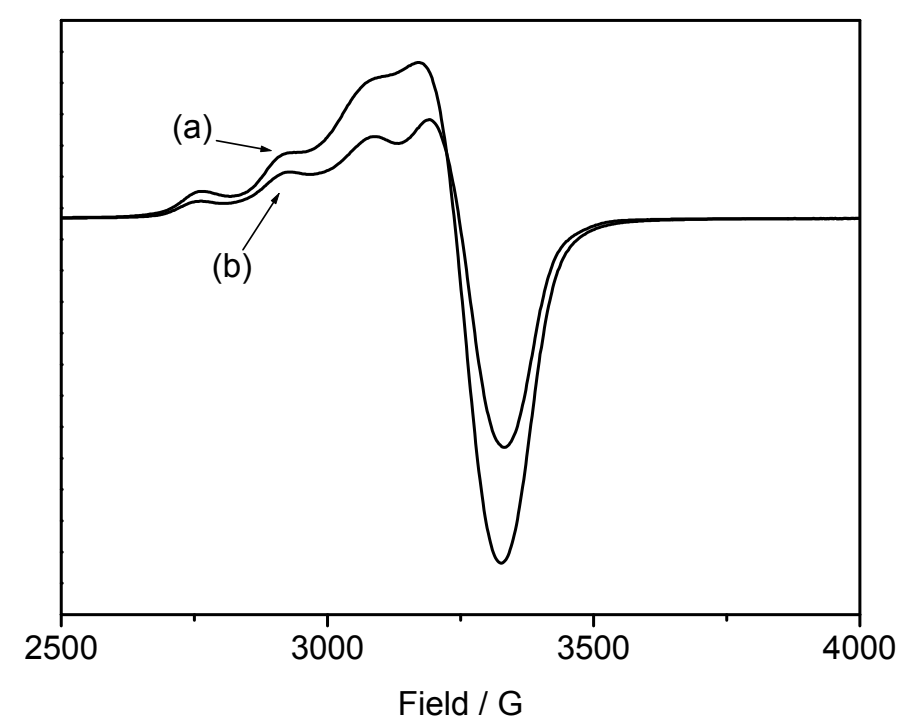

Figure 52. EPR spectra at $15 \mathrm{~K}$ : (a) frozen solution of the $\mathrm{Cu}^{\mathrm{II}} \mathrm{Zn}^{\mathrm{II}}$ model complex; (b) frozen solution of the $\mathrm{Cu}^{\mathrm{II}} \mathrm{Zn}^{\mathrm{II}}$ complex after addition of TMP.

A characteristic four-line pattern, expected for an isolated $d^{9} \mathrm{Cu}^{\mathrm{II}}$ ion, was observed in the EPR spectrum of the $\mathrm{Cu}^{\mathrm{II}} \mathrm{Zn}^{\mathrm{II}}$ complex, which confirmed the presence of the mixed metal species (the other possibly formed species, i.e. $\mathrm{Cu}^{\mathrm{II}} \mathrm{Cu}^{\mathrm{II}}$ and/or $\mathrm{Zn}^{\mathrm{II}} \mathrm{Zn}^{\mathrm{II}}$, are EPR silent at 15 $\mathrm{K})$. No significant changes were observed upon the addition of TMP substrate to the solution of the $\mathrm{Cu}^{\mathrm{II}} \mathrm{Zn}^{\mathrm{II}}$ complex, which strongly suggests formation of the copper(II)-phenolate adduct also with this dinuclear complex. Surprisingly, the colour of the solution changed from bluegreen to green without formation of the typical red colour of copper(II)-phenolate complexes. In this light, it can be assumed that the presence of the second copper(II) ion is necessary for the characteristic CT transition band to occur. Unfortunately, all attempts to crystallize the $\mathrm{Cu}^{\mathrm{II}} \mathrm{Zn}^{\mathrm{II}}$ species led to formation of the dicopper(II) or dizinc(II) complexes as crystalline compounds.

\subsection{Determination of the amount of coordinated phenol}

\subsubsection{General}

Job's Method, ${ }^{150}$ also called the Method of Continuous Variation, is a simple and effective approach to determine the stoichiometry for any chemical reaction with a general description as in $\mathrm{Eq}(1)$. 


$$
\mathrm{aA}+\mathrm{bB} \rightleftharpoons \mathrm{dD}
$$

This equation can be rewritten into the form of (2) by dividing all coefficients by "a".

$$
\mathrm{A}+\mathrm{nB} \rightleftharpoons \mathrm{mD}
$$

where $n=b / a$ and $m=d / a$.

Job's method is based on the following fact: if a series of solutions is prepared, each containing the same total number of moles of $A$ and $B$, but a different ratio $R$ of moles $B$ to moles $A$, the maximum amount of product $D$ is obtained in the solution in which $R=n$ (the stoichiometric ratio). This method is therefore useful and practical when information is sought about the formation of particular ligand-adducts of metal complexes or to deduce the maximum number of substrates B capable of coordinating to a given metal species A. To implement Job's Method experimentally, one prepares a series of solutions containing a fixed total number of moles of $\mathrm{A}$ and $\mathrm{B}$, but in which their ratio $\mathrm{R}$ is systematically varied from large to small, and then measures the amount of product obtained in each solution (e.g. via UV/vis spectroscopy). Then the amount of product (in case of UV/vis measurements represented by the absorption value) is plotted versus the molar fraction in B (b), and a maximum in the plot is obtained at the initially unknown value of $n$ (amount of the coordinated compound), that can be calculated with the simple equation $n=b / a$ (Table 15).

Table 15. Relation between $b$ value obtained experimentally from Job's plot and $n$ (amount of the coordinated compound).

\begin{tabular}{c|c}
\hline $\mathrm{b}$ & $\mathrm{n}$ \\
\hline 0.33 & 0.5 \\
0.50 & 1 \\
0.66 & 2 \\
0.75 & 3 \\
0.80 & 4 \\
\hline
\end{tabular}

Thus, for the present systems, a series of solutions was prepared by mixing different volumes of equimolar solutions of the two components (i.e. the appropriate dicopper complex $\mathrm{A}$ and phenol substrate B), followed by dilution to a constant volume to give solutions with identical total molar concentrations but different molar fractions. The UV/vis spectrum for each sample 
was then recorded. If a single, stable complex is formed, i.e. one that shows no appreciable dissociation, a plot of the absorbance versus the mole fraction of reactant B (phenol) gives a characteristic triangular correlation (Figure. 53 (1)). The mole fraction of the maximum of this plot, the apex of the triangle, indicates the molar composition of the formed complex. However, if a weak complex is formed, a very curved plot results (Figure. 53 (2)).

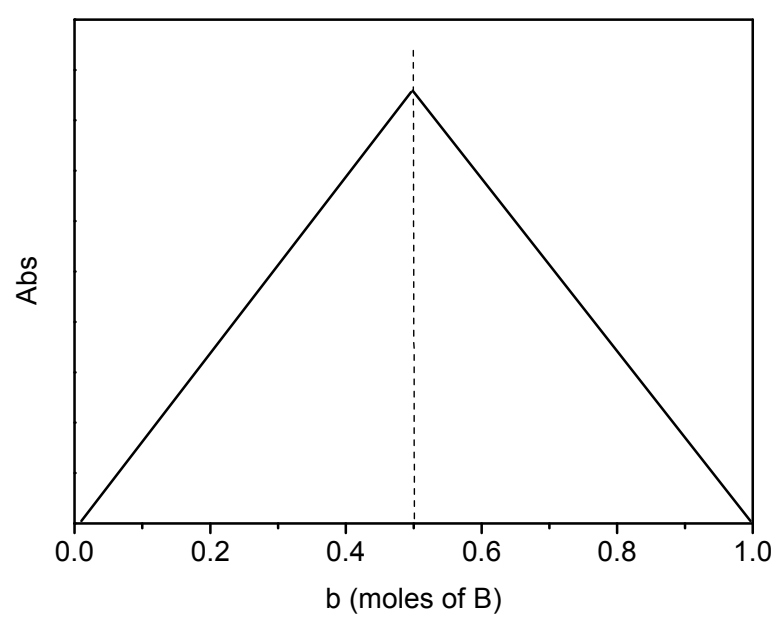

(1)

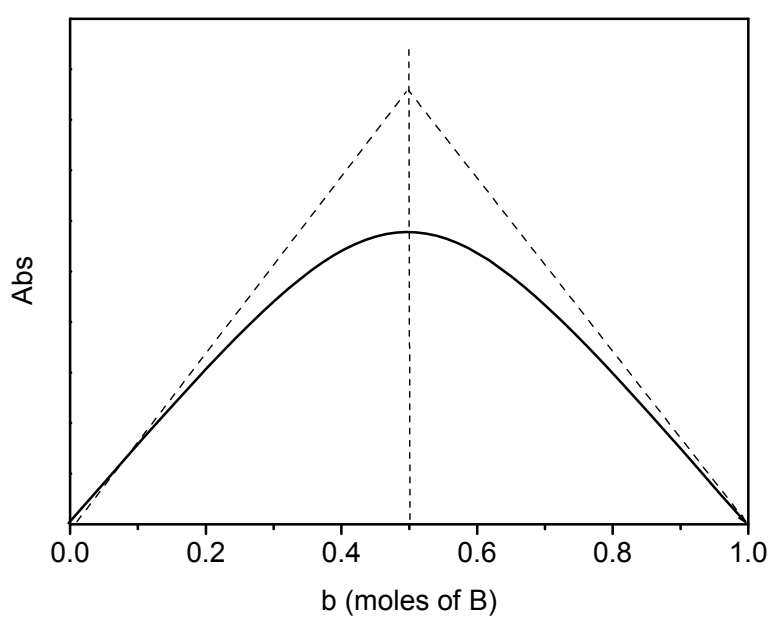

(2)

Figure 53. Schematic representation of Job's plots of stable (not dissociated) (1) and weak (2) complexes.

For a moderately stable complex, the molar composition of the complex can be obtained from the point of intersection of the tangents to the curve. However, for complexes where the dissociation constant $K_{d}$ (see Eq. (4)) is too large, the value for $n$ can not be determined very reliably using the continuous variations method as described by Job.

\subsubsection{Job plot of TMP with dicopper complex 5a}

Coordination of a phenol substrate like TMP to the dicopper complex 5a can be described with the equilibrium reaction given in Eq. (3). Methanolate, which serves as internal base, undergoes exchange with phenol substrate, resulting in release of a methanol molecule and formation of a dicopper(II)-phenolate species. However, depending on the reaction conditions, coordination of more than one phenol molecule is conceivable. 


$$
\left[\mathrm{LCu}_{2}(\mathrm{MeO}) \mathrm{MeOH}\right]^{2+}+\mathrm{n} \mathrm{PhOH} \rightleftharpoons\left[\mathrm{LCu}_{2}(\mathrm{PhO})_{n}(\mathrm{MeOH})\right]^{(3-n)+}+\mathrm{MeOH}
$$

Solutions of the dicopper complex and phenol used to perform a Job experiment were equimolar with concentrations equal to $\mathrm{C}_{0}$. Taking this into account, the maximum number of coordinated phenol molecules $n$ can be calculated accurately from the equation $n=b / a$ only when $K_{\mathrm{d}}$ (dissociation constant of phenolate adduct, Eq. (4)) is significantly smaller than $\mathrm{C}_{0}$ and can therefore be ignored in Eq. (5).

$$
\begin{gathered}
\mathrm{K}_{\mathrm{d}}=\frac{\left.\left[\mathrm{LCu}_{2}(\mathrm{MeO}) \mathrm{MeOH}\right]^{2+}\right][\mathrm{PhOH}]^{\mathrm{n}}}{\left.\left[\mathrm{LCu}_{2}(\mathrm{PhO})_{\mathrm{n}}\right]^{(3-\mathrm{n})+}\right]}=\frac{[\mathrm{A}][\mathrm{B}]}{[\mathrm{D}]} \\
\frac{\mathrm{b}}{\mathrm{a}}=\frac{\mathrm{K}_{\mathrm{d}}+\mathrm{nC}_{0}}{\mathrm{~K}_{\mathrm{d}}+\mathrm{C}_{0}} \sim \mathrm{n}
\end{gathered}
$$

Because of the further reactivity of the dicopper(II)-phenolate species formed after addition of TMP to 5a, determination of the stoichiometry for the C-C coupling reaction of TMP to yield TMSQ was performed using time-resolved stopped-flow UV/vis spectroscopy. In the presence of dioxygen, solutions of substrate and complex in $\mathrm{MeCN}$ (total concentration of 47 $\mathrm{mM}$ ) were mixed in different ratios, and the reaction was followed until the phenolate-tocopper LMCT band at $\lambda_{\max } 495 \mathrm{~nm}$ had reached its maximum. The corresponding absorption value for each mixture was plotted versus the molar fraction in phenol (b) (Figure 54). The maximum amount of TMP coordinated to the dicopper core of complex 5a was found to be 2 (Table 15). 


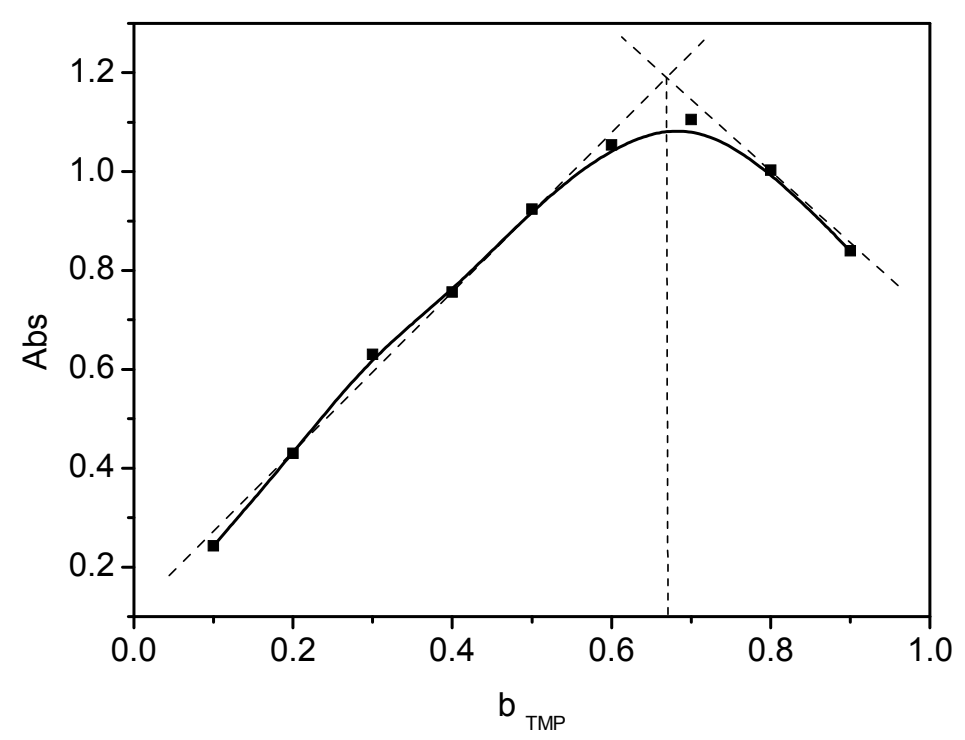

Figure 54. Job plot of TMP with dicopper complex 5a, $\lambda_{\max }=495 \mathrm{~nm}$.

The curved plot shows that the dicopper(II)- phenolate complex formed upon coordination of TMP within the bimetallic pocket exhibits fairly low overall stability owing to moderate binding of the phenolate (strong enough to accomplish appropriate activation of the total substrate fragment and therefore reactivity to occur, but weak enough to ensure that the product leaves after reaction and is thus isolable) and substantial dissociation occurs, which is indeed necessary to enable the further reaction steps with final formation of TMSQ.

The maximum number of coordinated TMP molecules indicated by the Job plot measurements requires careful consideration, since the copper-phenolate species is not very stable, and therefore the approximation $\mathrm{K}_{\mathrm{d}}<<\mathrm{C}_{0}$ and the corresponding simplification in Eq. (5) are invalid. Nevertheless, it can be assumed that coordination of two TMP molecules to the dicopper core in 5a requires some structural changes in the bimetallic core, which shows that catalytic oxidative C-C coupling of TMP to TMSQ is a complicated process.

In order to perform further studies on the formation of copper-phenolate species in solution and to gain knowledge about the driving force needed to accomplish activation of TMP as the key step for the reaction to occur, various phenols that are inert to any kind of oxidation reactions catalyzed by $\mathbf{5 a}$ were applied as model substrates. The binding of these phenols was proven by detection of the phenolate-to-copper LMCT band using UV/vis spectroscopy. Job's technique described above was applied in order to determine the stoichiometry upon binding of the model phenol substrates to the dicopper complex $\mathbf{5 a}$. 


\subsubsection{Job plot of 4-methylphenol and 4-tert-butylphenol with dicopper complex 5a}

Initial model studies on the binding of phenol substrates to the dicopper core in 5a were performed using $p$-cresol (4-MeP), an analogue of TMP. Addition of $p$-cresol to a green solution of 5a in $\mathrm{MeCN}$ led to a visible colour change of the solution from green to red with appearance of an LMCT band at $\lambda_{\max } 457 \mathrm{~nm}$ in the UV/vis spectrum. No spectroscopic changes were observed after storing the red solution in air for one week, which confirmed the "inert nature" of 4-MeP in any kind of transformation in the presence of $\mathbf{5 a}$.

This unreactivity of p-cresol compared to DMP and TMP, particularly in the coppercatalyzed oxidative para C-C coupling, could be explained by the varying number of electrondonating $\mathrm{CH}_{3}$-groups on the aromatic ring of each phenol, which are most likely responsible for activation of the substrate while coordinated to the dicopper core in 5a. Additional $\mathrm{CH}_{3^{-}}$ groups not only ensure a stronger binding of the phenolate to the copper ion (as reflected in the $\lambda_{\max }$ section 7.3) but also enhance the formation of a reactive species. Thus, for any further reactivity to occur (formation of $C$-C coupled product) the presence of at least two $\mathrm{CH}_{3}$-groups on the phenol substrate is required.

The Job plot of 4-MeP (as well as other model phenol substrates) with 5a was performed in a slightly different manner compared to the previous experiments described in this chapter. The initial solution of dicopper complex 5a in $\mathrm{MeCN}$ was diluted further in a stepwise manner (each dilution representing a certain complex to phenol ratio $\mathrm{R}$ ) by addition of a solution of phenol with a constant concentration. Thus, for the Job plot of 4-MeP with 5a, solutions with a concentration of $48 \mathrm{mM}$ were used and the UV/vis spectrum of the mixture was recorded after each dilution step (Figure 55). It has to be mentioned that a dilution effect for the band of interest $\left(\lambda_{\max }=457 \mathrm{~nm}\right)$ could be observed during the experiment as reflected in UV/vis spectrum, but only after the maximum absorbance (and therefore the maximum number of coordinated phenol) of the dicopper(II)-phenolate species was reached. 




Figure 55. UV/vis spectrum of the mixture after each dilution step: green line (initial dicopper complex 5a); red line (solution of $\mathbf{5 a}$ and 4-MeP with a maximum absorbance).

From the Job plot depicted in Figure 56 it can be deduced that a maximum of two molecules of 4-MeP can be coordinated to the dicopper core of complex $\mathbf{5 a}$, similar to the situation with TMP.

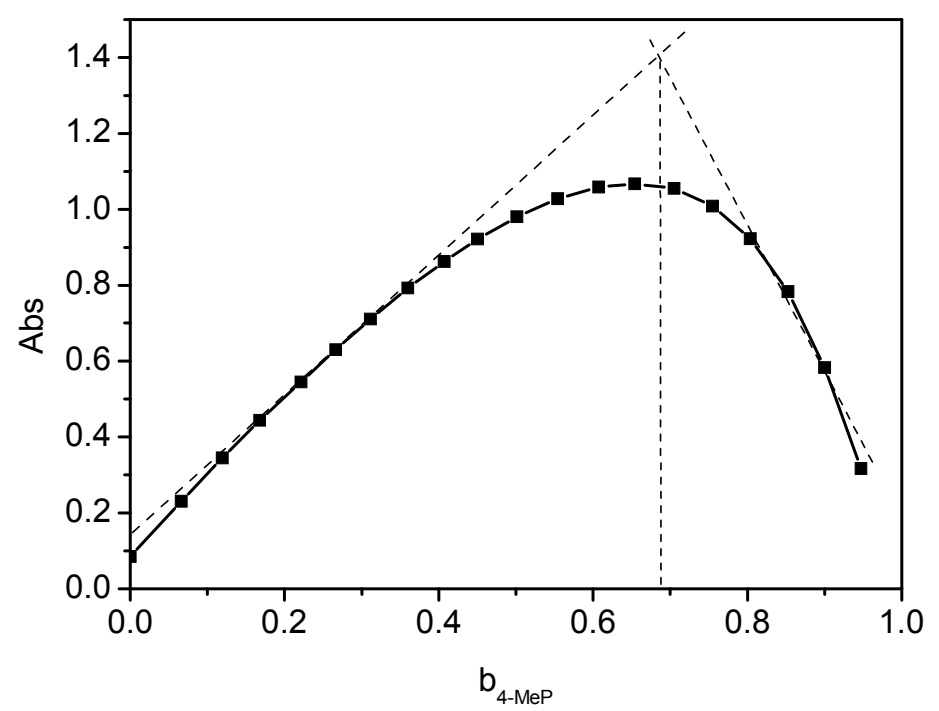

Figure 56. Job plot of $p$-cresol with dicopper complex 5a, $\lambda_{\max }=457 \mathrm{~nm}$.

A Job plot of 4-tert-butylphenol $t \mathrm{BuP}$ with 5a was performed using $49 \mathrm{mM}$ solutions of both components. The corresponding absorption value of the LMCT band $\left(\lambda_{\max }=455 \mathrm{~nm}\right)$ for each mixture after dilution was plotted versus the molar fraction in $t \mathrm{BuP}$ (b) (Figure 57). The 
maximum number of $t \mathrm{BuP}$ molecules coordinated to the dicopper core of complex 5a was also found to be two.

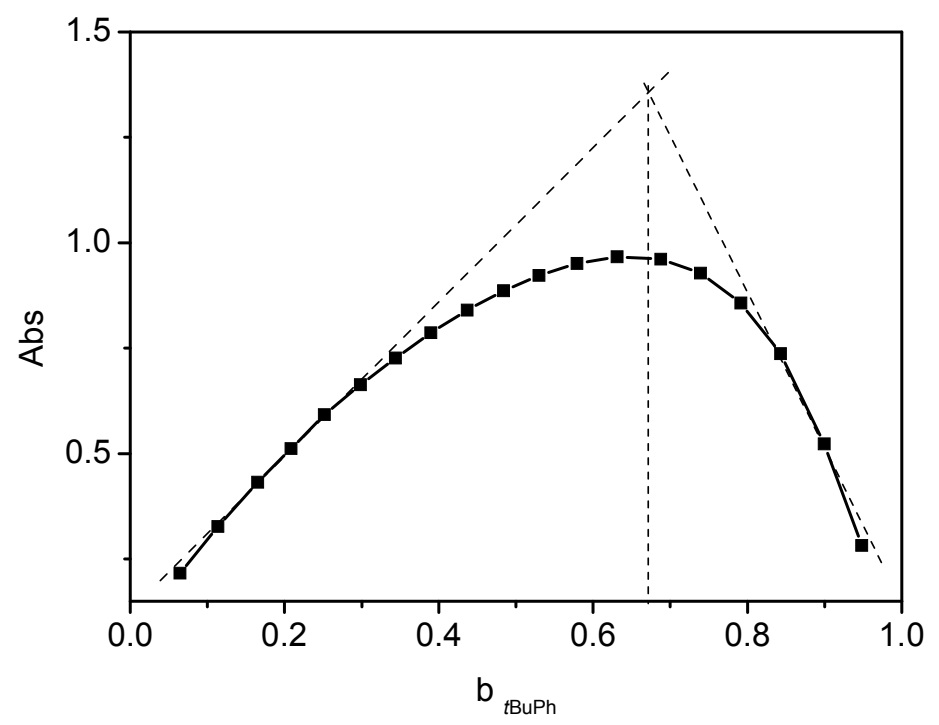

Figure 57. Job plot of $t$ BuP with dicopper complex 5a, $\lambda_{\max }=455 \mathrm{~nm}$.

The curved shape for this Job plot - as in the case of 4-MeP - shows that the dicopperphenolate complex formed upon coordination of $t \mathrm{BuP}$ to the dicopper complex exhibits rather low stability, but in contrast to TMP this low stability does not induce any further reactivity and results only in dissociation of the phenolate species. This became evident when all attempts to crystallize any of the observed alkyl-substituted dicopper-phenolate species only led to regeneration and isolation of the initial dicopper(II) complex 5a, indicative of significant dissociation of the phenolate species under these conditions.

Based on these results, it can be assumed that a key factor required for further reaction to occur is not the number of coordinated phenol substrates, as the Job plot experiments have indicated the same values for TMP, 4-MeP and $t \mathrm{BuP}$, but the electronic and steric properties of the substituents on the phenol ring.

\subsubsection{Job plot of 4-hydroxybenzamide and pentafluorophenol with dicopper complex} $5 a$

Further studies on the binding of phenol molecules within the dicopper pocket of 5a were done using phenols with electron-withdrawing groups, like pentafluorophenol (PFP) and 4- 
hydroxybenzamide (4-AP). Addition of 4-AP to a solution of 5a in $\mathrm{MeCN}$ led to the formation of a yellow-green solution with an LMCT band at $\lambda_{\max } 446 \mathrm{~nm}$, which is in sharp contrast to the previously described observations after phenol addition, where red solutions were obtained. The highest absorption value reached in the Job plot experiment corresponded to a complex with a 1 to 1 ratio of 4-AP and $\mathbf{5 a}$ (Figure 58).

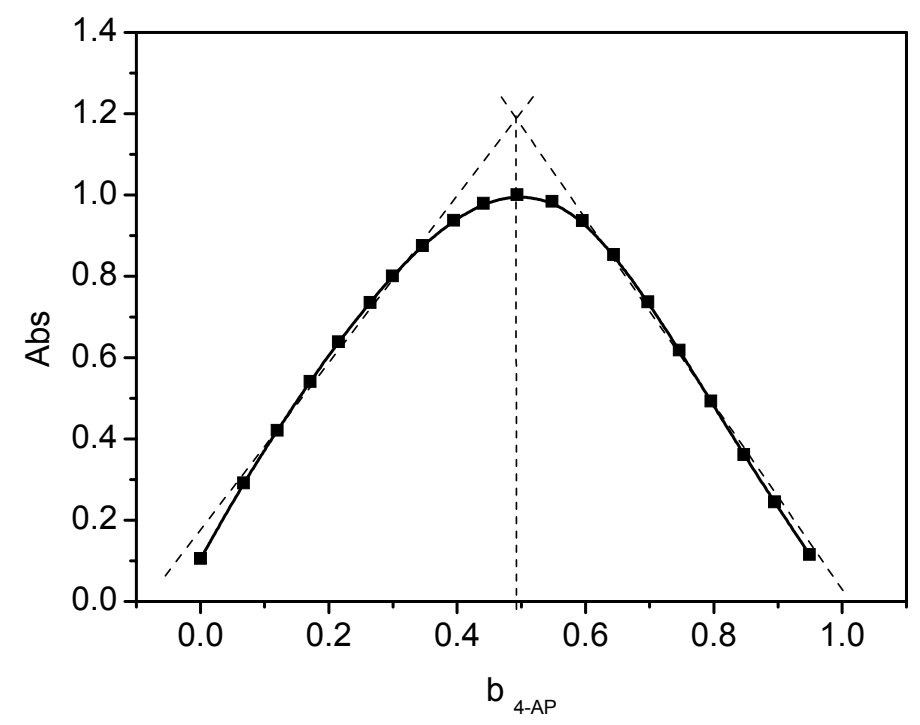

Figure 58. Job plot of 4-AP with dicopper complex 5a, $\lambda_{\max }=446 \mathrm{~nm}$.

In this case only one phenol molecule is able to coordinate to the dicopper core. The slightly curved plot (the deviation from a perfect triangular shape is significantly smaller than for any of the phenols discussed in section 7.5.3) shows that the dicopper phenolate complex exhibits moderate stability and dissociation of the phenolate species is still likely to occur to some degree in solution. Nonetheless, crystallization of a solution of 5a with excess of 4-AP in $\mathrm{MeCN}$ by slow diffusion with $\mathrm{Et}_{2} \mathrm{O}$ led to the formation of a crystalline compound. Single crystals were used for an X-ray crystallographic analysis and the formation of a complex with only one coordinated 4-AP molecule was unambiguously proven (see section 8.2 ).

The UV/vis spectrum of the yellow-green solution formed after addition of PFP to dicopper complex 5a in MeCN showed an intense CT band at $\lambda_{\max } 397 \mathrm{~nm}$. Formation of a very stable copper-pentafluorophenolate complex was evidenced by a Job's experiment, as the corresponding plot of absorbance versus the mole fraction $b$ of PFP featured the characteristic triangular correlation (Figure 59). The optimal value of $b \sim 0.5$ implies that this stable complex contains only one coordinated pentafluorophenolate molecule. 




Figure 59. Job plot of PFP with dicopper complex 5a, $\lambda_{\max }=397 \mathrm{~nm}$.

Single crystals of this phenolate adduct were obtained and coordination of only one PFP molecule was unequivocally proven by X-ray crystallography (see section 8.2 ).

A set of Job experiments carried out with different phenols as substrate molecules clearly showed that the deciding factor for C-C oxidative coupling to occur - after coordination of the phenol substrate has taken place - is not the number of coordinated phenols, but the electronic properties of the substituents on the phenol ring. Moreover, stable (mono)phenolate adducts could be isolated and even crystallized in case of strong electron-withdrawing substituents on the phenol ring.

\subsection{Mechanistic studies of C-C coupling of TMP under anaerobic conditions}

The catalytic oxidative C-C coupling of TMP to yield TMSQ, catalyzed by dicopper complex 5a and with dioxygen as the oxidant, is an overall 6-electron process ( $3 \mathrm{e}^{-}$per TMP molecule) which implies a rather complicated overall reaction pathway. The formation of TMSQ as the final product is expected to proceed through a series of intermediate species, each of which presumably requires the formation of an at least partially reduced dicopper species. The proposed reaction pathway is described in more detail in section 12. Therefore, initial mechanistic studies were performed under anaerobic conditions with the aim to determine the 
role of dioxygen in the oxidative C-C coupling of TMP and to trap possible intermediates (both organic compounds and copper species).

\subsubsection{UV/vis studies under $\mathrm{N}_{2}$-atmosphere}

Addition of TMP to a green solution of $5 \mathbf{a}$ (Figure 60 (a)) under an $\mathrm{N}_{2}$-atmosphere led to the same spectroscopic changes as for the analogous reaction in the presence of dioxygen, i.e. instantaneous formation of a red solution with concomitant appearance of an intense LMCT band at $\lambda_{\max } 495 \mathrm{~nm}\left(\varepsilon \sim 1500 \mathrm{~L} \cdot \mathrm{mol}^{-1} \cdot \mathrm{cm}^{-1}\right)$ and a shift of the $\mathrm{Cu}$-based $d$ - $d$ transition band from $989 \mathrm{~nm}$ to $960 \mathrm{~nm}$ (Figure 60 (b)). This shift indicates slight geometric changes of the copper ion/ions from trigonal bipyramidal to square planar geometry upon substitution of methanolate for phenolate within the bimetallic pocket.

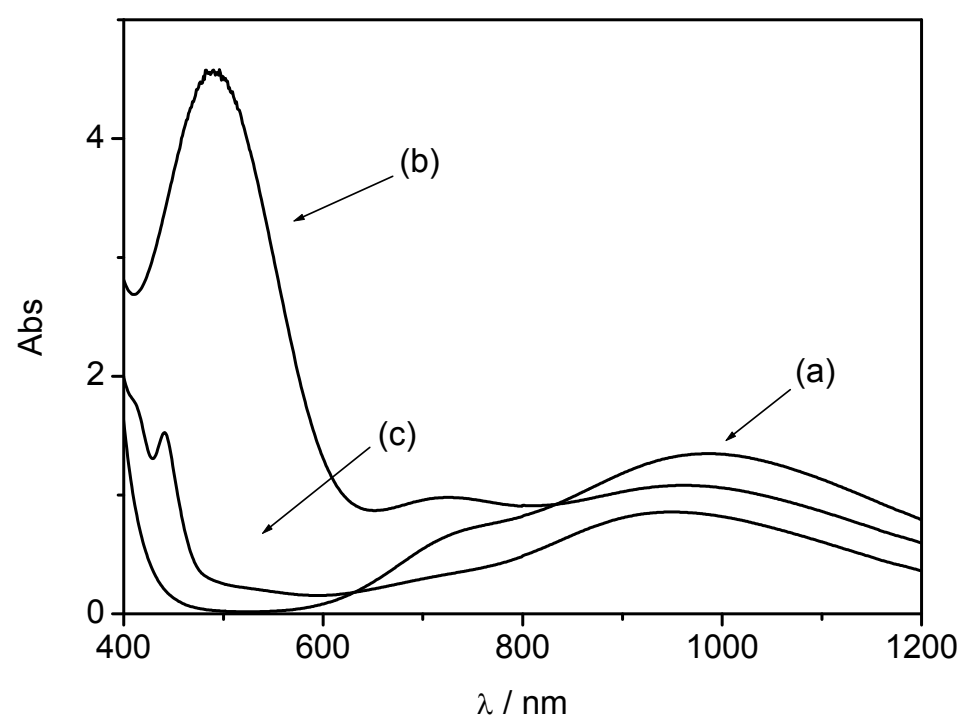

Figure 60. UV/vis spectra at different stages of the oxidative coupling of TMP under anaerobic conditions (initial concentration of $\mathbf{5 a}=0.005 \mathrm{~mol} \cdot \mathrm{L}^{-1}$; initial concentration of TMP $=0.025 \mathrm{~mol} \cdot \mathrm{L}^{-1}$; solvent: $\mathrm{MeCNCN} / \mathrm{CH}_{2} \mathrm{Cl}_{2} 4: 6 \mathrm{v} / \mathrm{v}$ ): (a) starting solution of $\mathbf{5 a}$; (b) a few minutes after addition of TMP (red solution); (c) $48 \mathrm{~h}$ after addition of TMP (only traces of TMSQ are observed).

Complete disappearance of the charge-transfer band was observed within $48 \mathrm{~h}$, concomitant with formation of a yellow-green solution with a $\mathrm{Cu}^{\mathrm{II}}$-based $d-d$ transition band at $\lambda_{\max } 947$ nm. Only traces of the TMSQ product were detected; this product has a very large extinction 
coefficient $\varepsilon$ of $c a .96000 \mathrm{~L} \cdot \mathrm{mol}^{-1} \cdot \mathrm{cm}^{-1}$ and therefore even traces already show up quite pronounced in the UV/vis spectrum (Figure 60 (c)). The yellow-green solution formed after this first reaction step can be stored under an $\mathrm{N}_{2}$-atmosphere indefinitely, without any observable spectroscopic and visual changes. For any further oxidation reaction to occur the presence of dioxygen is required. Addition of $\mathrm{O}_{2}$ induced an instantaneous colour change from yellow-green to the initially observed red colour and the product TMSQ started to form 10 minutes after introducing dioxygen to the catalytic system.

It was deemed interesting to study the coordination of TMP within the bimetallic pocket of $\mathbf{5 a}$ in the absence of oxygen, since in this case the reaction can be halted at the first intermediate stage and only stoichiometric processes can take place.

\subsubsection{Job plot of TMP with 5 a under anaerobic conditions}

Determination of the stoichiometry for the reaction of TMP with dicopper complex 5a in the absence of dioxygen was performed using time-resolved stopped-flow UV/vis spectroscopy. Solutions of the TMP substrate and the complex in MeCN $(50 \mathrm{mM})$ were prepared in an $\mathrm{N}_{2}$ filled glove box and then mixed in different ratios; and the reaction was followed until the LMCT band at $495 \mathrm{~nm}$ had reached its maximum (after 9 min for each sample). Absorption maxima for each mixture were plotted versus the molar fraction in TMP (Figure 61). In contrast to the results obtained from the Job plot experiments in the presence of $\mathrm{O}_{2}$, where two TMP molecules were found to be interacting with dicopper complex 5a, the maximum number of TMP molecules coordinating to $\mathbf{5 a}$ under an $\mathrm{N}_{2}$-atmosphere was found to be only one. 


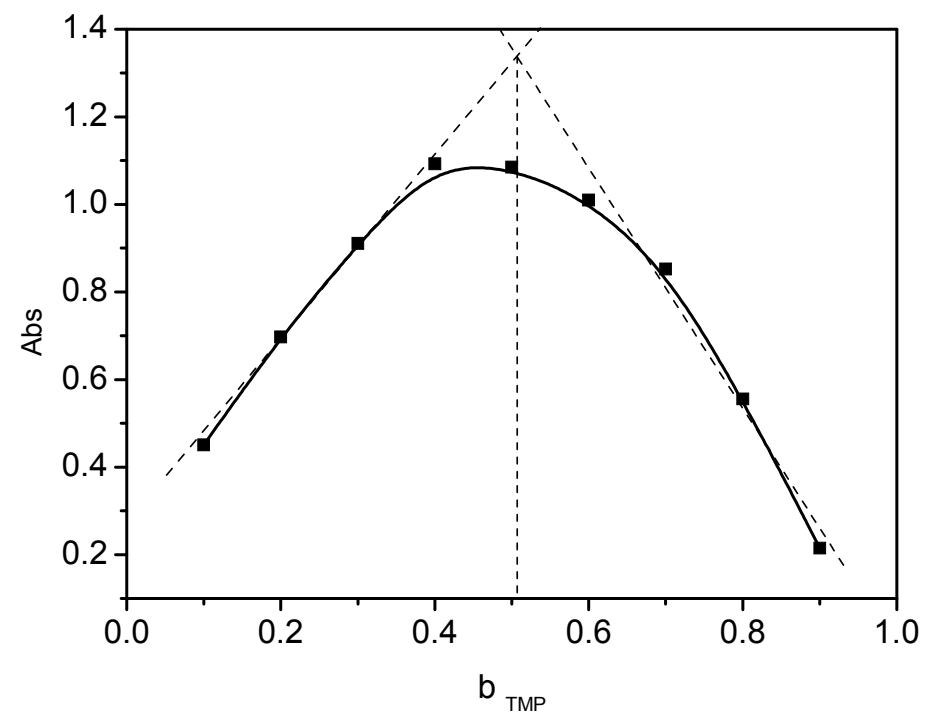

Figure 61. Job plot of TMP with 5a under anaerobic conditions, $\lambda_{\max }=495 \mathrm{~nm}$.

From these studies it can be concluded that coordination of TMP within the bimetallic pocket of 5a occurs under anaerobic conditions as well, while further formation of TMSQ can take place only in the presence of dioxygen. Disappearance of the charge-transfer band and formation of the yellow-green solution indicate the complete absence of the initially formed copper-phenolate species after $48 \mathrm{~h}$ of reaction under anaerobic conditions, which is clear evidence for its participation in the oxidative coupling reaction.

\subsubsection{Trapping of the intermediates under anaerobic conditions}

The observation that under anaerobic conditions the reaction can be halted at what appears to be an intermediate stage of the overall oxidative coupling reaction, encouraged to attempt the synthesis of this organic intermediate on a sufficiently large scale to allow its isolation and characterization. Thus, $250 \mathrm{mmol}$ of TMP and $50 \mathrm{mmol}$ of dicopper complex $\mathbf{5 a}$ were reacted under an inert atmosphere to generate, after flash chromatography, a white powder. The product could be identified as 4,4'-dihydroxy-3,3',5,5' -tetramethylbibenzyl (TMBB) (Scheme $35)$. 


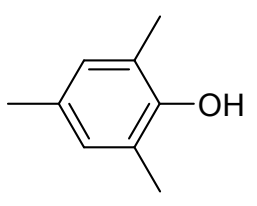

TMP

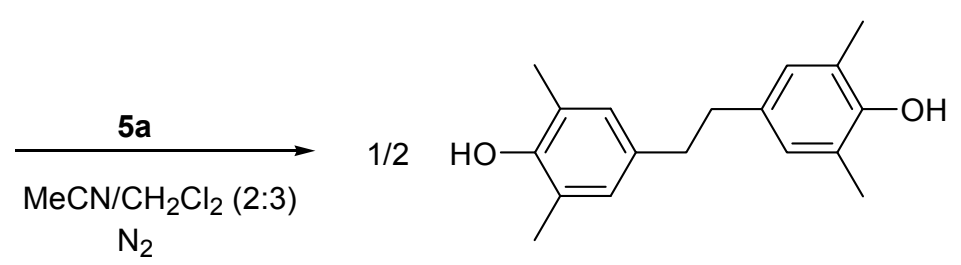

TMBB

Scheme 35. C-C coupling of TMP catalyzed by $\mathbf{5 a}$ under an $\mathrm{N}_{2}$-atmosphere.

This overall $2 \mathrm{e}^{-}$process (based on the product) proceeds stoichiometrically via abstraction of an electron from TMP by the dicopper system. In this case only one out of the five equivalents of TMP used in the reaction can undergo a chemical transformation to yield TMBB, which was indeed confirmed by ${ }^{1} \mathrm{H}$ NMR studies. Around $80 \%$ (4 equivalents) of TMP was found to be unreacted after the first reaction step in the glove box and the other $20 \%$ ( 1 equivalent) underwent $\mathrm{C}-\mathrm{C}$ coupling to form TMBB in 100\% yield based on redox equivalents corresponding to formation of the dicopper complex in its $\mathrm{Cu}^{\mathrm{I}} \mathrm{Cu}^{\mathrm{II}}$ state. This intermediate organic species (when isolated) is stable in air and can be further oxidized to TMSQ by oxygen only in the presence of the original dicopper complex 5a: upon exposure of TMBB to $\mathrm{O}_{2}$ in the presence of 5a the initially observed charge-transfer band $\left(\lambda_{\max }=495 \mathrm{~nm}\right)$ reappeared in the UV/vis spectrum and instantaneous formation of TMSQ was observed.

In order to spectroscopically prove the formation of a dinuclear mixed-valence $\mathrm{Cu}^{\mathrm{I}} \mathrm{Cu}^{\mathrm{II}}$ species after formation of TMBB under an $\mathrm{N}_{2}$-atmosphere, an independently synthesized model $\mathrm{Cu}^{\mathrm{I}} \mathrm{Cu}^{\mathrm{II}}$ complex was used for comparison. A solution of $\mathrm{HL}^{4}$ in a mixture of $\mathrm{MeCN} / \mathrm{CH}_{2} \mathrm{Cl}_{2}$ was treated with 1 equivalent of $\mathrm{KO} t \mathrm{Bu}$ to deprotonate the pyrazole $\mathrm{NH}$-unit, and 1 equivalent of both $\mathrm{Cu}\left(\mathrm{ClO}_{4}\right)_{2} \cdot 6 \mathrm{H}_{2} \mathrm{O}$ and $\left[\mathrm{Cu}(\mathrm{MeCN})_{4}\right]\left(\mathrm{ClO}_{4}\right)$ were subsequently added, yielding a light-green solution. The UV/vis spectrum of the resulting solution, with an equal concentration as for the experiment performed with 5a, was recorded in an inert atmosphere (Figure $62(\mathrm{c})$ ). 


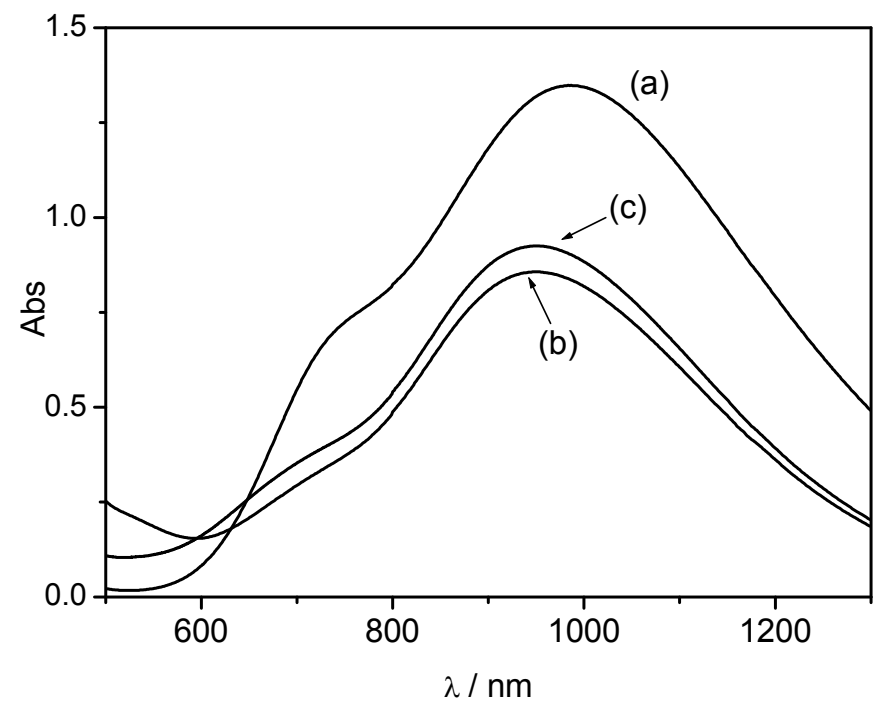

Figure 62. UV/vis spectra under anaerobic conditions: (initial concentration of $\mathbf{5 a}=0.005$ $\mathrm{mol} \cdot \mathrm{L}^{-1}$; initial concentration of TMP $=0.025 \mathrm{~mol} \cdot \mathrm{L}^{-1}$; solvent: $\mathrm{MeCN} / \mathrm{CH}_{2} \mathrm{Cl}_{2}$ 4:6 v/v): (a) starting solution of $\mathbf{5 a}$; (b) $48 \mathrm{~h}$ after addition of TMP; (c) $0.005 \mathrm{~mol} \cdot \mathrm{L}^{-1}$ solution of the model $\mathrm{Cu}^{\mathrm{I}} \mathrm{Cu}^{\mathrm{II}}$ complex).

The overall spectral features of the solution of the model $\mathrm{Cu}^{\mathrm{I}} \mathrm{Cu}{ }^{\mathrm{II}}$ complex, and most notably the absorption maximum at $\lambda_{\max } 947 \mathrm{~nm}$ (as well as the approximate value for the extinction coefficient), are very similar to those observed in the UV/vis spectrum of the yellow-green solution formed after reaction and storage for $48 \mathrm{~h}$ in the nitrogen filled glove box.

Therefore, on the basis of these results, the yellow-green solution was characterized as a mixture of stoichiometrically formed TMBB and mixed-valence $C u^{I} C u^{I I}$ complex, generated by partial reduction of the original dicopper(II) complex $\mathbf{5 a}$.

\subsubsection{Evidence for the formation of a mixed-valence $\mathrm{Cu}{ }^{\mathrm{I}} \mathrm{Cu}{ }^{\mathrm{II}}$ species}

Formation of the mixed-valence $\mathrm{Cu}^{\mathrm{I}} \mathrm{Cu}^{\mathrm{II}}$ species after $48 \mathrm{~h}$ of storage in an $\mathrm{N}_{2}$-atmosphere was also proven by means of EPR spectroscopy. Copper(II) is especially amenable to EPR investigation, because it has only one unpaired electron and in addition, the ${ }^{63} \mathrm{Cu}$ and ${ }^{65} \mathrm{Cu}$ (nuclear spin $3 / 2$ ) nuclei give rise to a characteristic four-line pattern because of electronnuclei-spin interaction, denoted by the hyperfine parameter $\mathrm{A}$. 
The initial dicopper(II) complex 5a is EPR-silent (under the low temperature conditions used for the measurements: $15 \mathrm{~K}$ ) due to the strong antiferromagnetic interaction (see section 5.4.4 and 7.4.2) between the two copper ions, brought about by the bridging pyrazolate unit. On this basis, it can be reasoned that a dinuclear mixed-valence $\mathrm{Cu}^{\mathrm{I}} \mathrm{Cu}^{\mathrm{II}}$ species should be detectable at low temperature.

First of all, the X-band EPR spectroscopic measurement of a frozen solution of the independently synthesized $\mathrm{Cu}^{\mathrm{I}} \mathrm{Cu}^{\mathrm{II}}$ model complex in $\mathrm{MeCN}$ glass was recorded at $15 \mathrm{~K}$ (Figure $63(\mathrm{a})$ ).



Figure 63. EPR spectra at $15 \mathrm{~K}$ : (a)-red line, frozen solution of the $\mathrm{Cu}^{\mathrm{I}} \mathrm{Cu}^{\mathrm{II}}$ model complex; (b)-green line, simulated spectrum.

The red line (a) represents the recorded EPR spectrum and the green line (b) is the corresponding simulated spectrum. A typical four-line pattern is observed, which is characteristic for a non-perturbed $\mathrm{Cu}^{\mathrm{II}}$ ion. Since under the conditions applied for these measurements any other copper species that is possibly present, i.e. traces of $\mathrm{Cu}^{\mathrm{II}} \mathrm{Cu}^{\mathrm{II}}$ and/or $\mathrm{Cu}^{\mathrm{I}} \mathrm{Cu}^{\mathrm{I}}$ - both species can in principle have formed during the direct synthesis of the mixedvalence $\mathrm{Cu}^{\mathrm{I}} \mathrm{Cu}^{\mathrm{II}}$ complex - would be EPR-silent, this signal was attributed to the mixedvalence copper species. The data for the simulated spectrum revealed $g_{I I}(2.25)>g \perp(2.06)$ and $\left|\mathrm{A}_{I I}\right|=157 \mathrm{G}$ are typical of a five-coordinate square pyramidal structure around $\mathrm{Cu}^{\mathrm{II}}$ ion. ${ }^{151} \mathrm{At}$ the same time, the overall UV/vis features of the solution of the model $\mathrm{Cu}^{\mathrm{I}} \mathrm{Cu}^{\mathrm{II}}$ complex are characteristic for a copper ion in trigonal bipyramidal geometry. The relation between observed EPR and UV/vis spectroscopic features can not be explained at the present moment. 
The EPR spectrum of the reaction mixture after storage in glove box was recorded under the same conditions and it is depicted in Figure 64 (c) together with the EPR spectrum of the model $\mathrm{Cu}^{\mathrm{I}} \mathrm{Cu}^{\mathrm{II}}$ complex Figure 64 (a). The same four-line pattern as for the model complex was observed. It has to be mentioned that the shape of the spectrum of the reaction mixture (c) indicates the presence of at least two different mixed-valence species in solution with most likely similar features, possibly due to the presence of TMBB or excess TMP, and therefore simulation was not performed.

The single most important conclusion that can be drawn from these measurements is that the unprecedented formation of a mixed-valence $\mathrm{Cu}^{\mathrm{I}} \mathrm{Cu}^{\mathrm{II}}$ species, brought about by the reduction of one of the copper ions in complex 5a, is clearly observable after the first reaction step, i.e. the oxidative C-C coupling of two molecules of TMP to yield TMBB. This first step does proceed under anaerobic conditions.

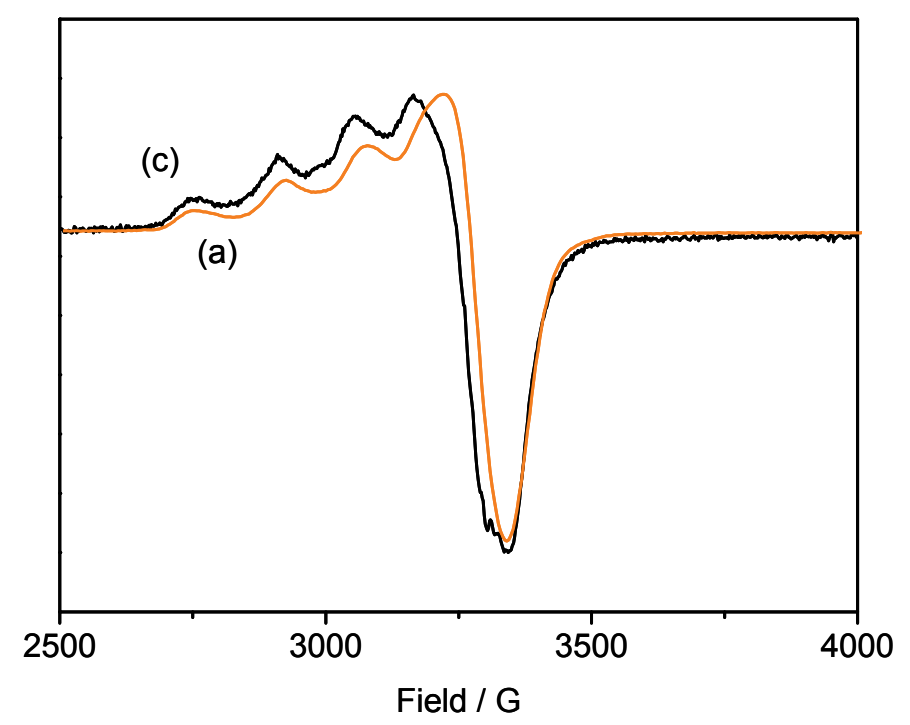

Figure 64. EPR spectra at $15 \mathrm{~K}$ : (a)-red line, frozen solution of the model $\mathrm{Cu}^{\mathrm{I}} \mathrm{Cu}^{\mathrm{II}}$ complex; (c)-black line, yellow-green solution after $48 \mathrm{~h}$ of storage in glove box.

To further investigate the nature of the copper species generated under anaerobic conditions, and after formation of the organic product TMBB was complete, it was first attempted to obtain suitable crystals from the reaction medium. Unfortunately, under these conditions (direct crystallization from the reaction mixture after first reaction step under anaerobic conditions), only green powder was formed. To enhance the stability of the relevant copper complex formed after the oxidative coupling and to trap proposedly a mixed-valence $\mathrm{Cu}^{\mathrm{I}} \mathrm{Cu}^{\mathrm{II}}$ species, two equivalents of tert-butyl isocyanide (per dicopper complex) were added to the 
obtained yellow-green solution (see above). Addition of the strong $\sigma$-donor isocyanide coligand led to the disappearance of the typical four-line pattern for copper(II) (Figure 65 (d)).

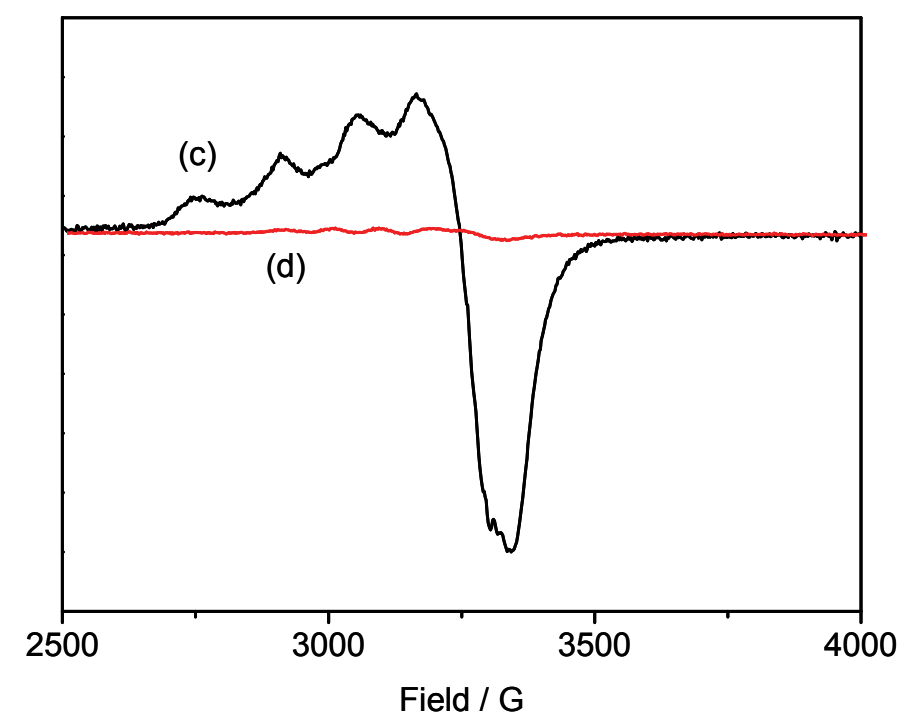

Figure 65. EPR spectra at $15 \mathrm{~K}$ : (a)-black line, yellow-green frozen solution after $48 \mathrm{~h}$ of storage in glove box; (c)-red line, yellow-green solution after addition of $\left(\mathrm{CH}_{3}\right)_{3} \mathrm{CNC}$.

Coordination of the strong $\sigma$-donor isocyanide ligand was implied by the colour change to intense green. Isolation of the complex was achieved by slow diffusion of the acetonitrile solution with $\mathrm{Et}_{2} \mathrm{O}$. Characterization by IR spectroscopy revealed the presence of two bands at $v_{\mathrm{CN}} 2173$ and $2151 \mathrm{~cm}^{-1}$, indicative of two isocyanide ligands on a $\mathrm{Cu}^{\mathrm{I}}$ center. Molecular structure of the complex formed was determined by X-ray crystallography. Figure 66 shows the molecular structure of complex 9 , while Table 16 contains selected data for intramolecular atom distances and angles. The anticipated mixed-valence $\mathrm{Cu}^{\mathrm{I}} \mathrm{Cu}^{\mathrm{II}}$ motif is clearly preserved in this structure, but instead of the expected dinuclear species, this complex exists in the related dimeric form in the solid state. Such a dimerisation has most probably taken place due to the coordination of the strong $\sigma$-donor isocyanide co-ligands which induces a rearrangement of the mixed-valence dinuclear $\mathrm{Cu}^{\mathrm{I}} \mathrm{Cu}^{\mathrm{II}}$ core and coordination of the $\mathrm{Cu}^{\mathrm{I}}$ ions to the dangling chelate side arms, concomitant with formation of the central bis( $\mu$-pyrazolato) dicopper(II) core. These two copper(II) ions reside in the complex in the same coordination mode as in 6, which is reflected by an almost perfect trigonal bipyramidal $\left\{\mathrm{N}_{5}\right\}$ coordination environment $(\tau=0.98)$, where one of the apical $\mathrm{Cu}-\mathrm{N}$-bonds is slightly elongated, with a $d(\mathrm{Cu} 1-\mathrm{N} 3)$ of $2.12 \AA$. 


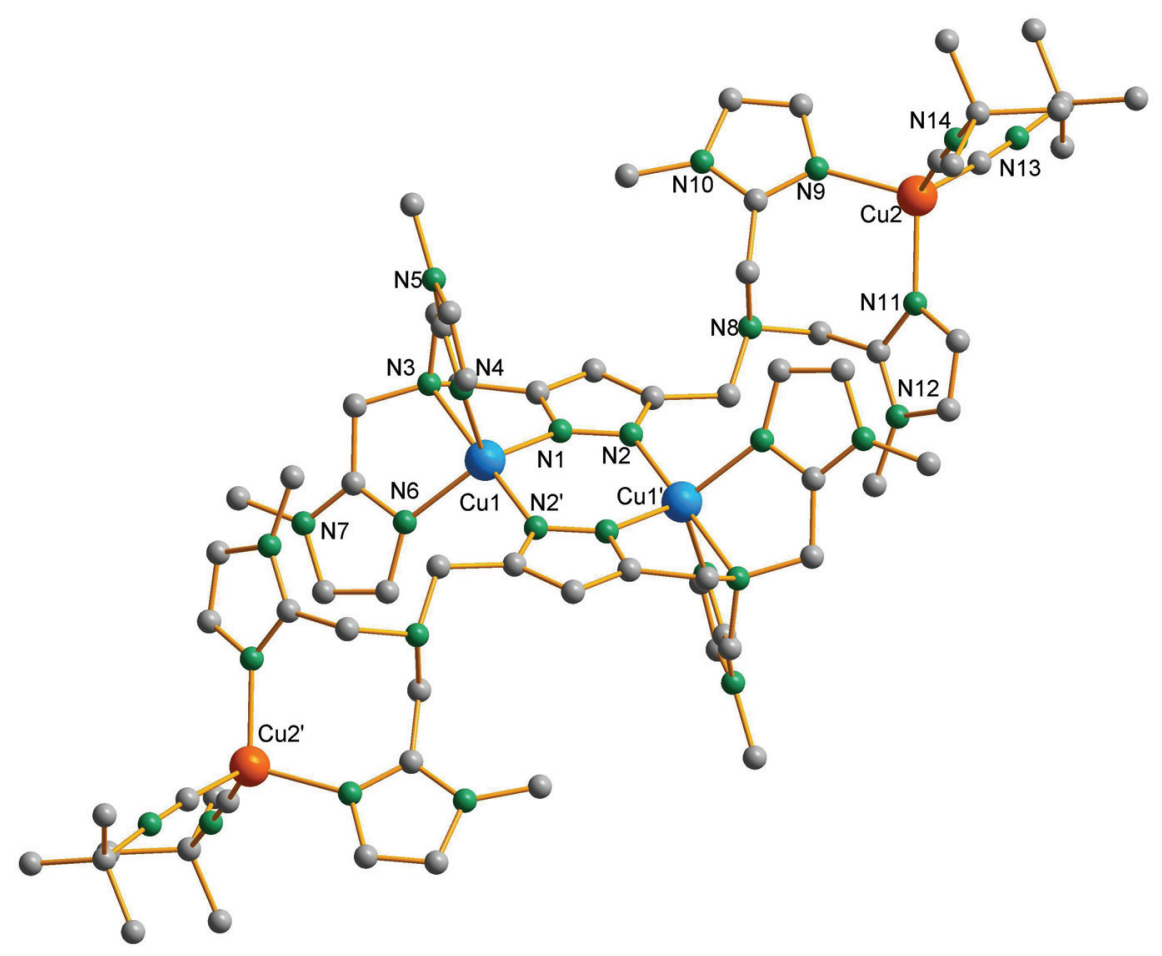

Figure 66. Molecular structure of the cation of 9.

Table 16. Selected intramolecular distances $(\AA)$ and angles $\left(^{\circ}\right)$ for complex 9.

\begin{tabular}{llll}
\hline \multicolumn{3}{c}{ Distances } \\
\hline $\mathrm{Cu}(1)-\mathrm{N}(2)^{\prime}$ & $1.946(3)$ & $\mathrm{Cu}(2)-\mathrm{C}(31)$ & $1.939(5)$ \\
$\mathrm{Cu}(1)-\mathrm{N}(1)$ & $2.025(3)$ & $\mathrm{Cu}(2)-\mathrm{N}(11)$ & $2.060(3)$ \\
$\mathrm{Cu}(1)-\mathrm{N}(4)$ & $2.071(4)$ & $\mathrm{Cu}(2)-\mathrm{N}(9)$ & $2.077(4)$ \\
$\mathrm{Cu}(1)-\mathrm{N}(6)$ & $2.075(4)$ & $\mathrm{Cu}(1) \cdots \mathrm{Cu}(1)$, & $3.847(1)$ \\
$\mathrm{Cu}(1)-\mathrm{N}(3)$ & $2.121(3)$ & & \\
$\mathrm{Cu}(2)-\mathrm{C}(26)$ & $1.924(4)$ & & \\
\hline & & & \\
\hline $\mathrm{N}(2)^{\prime}-\mathrm{Cu}(1)-\mathrm{N}(1)$ & $101.59(13)$ & $\mathrm{N}(4)-\mathrm{Cu}(1)-\mathrm{N}(3)$ & $79.30(14)$ \\
$\mathrm{N}(2)^{\prime}-\mathrm{Cu}(1)-\mathrm{N}(4)$ & $97.81(14)$ & $\mathrm{C}(26)-\mathrm{Cu}(2)-\mathrm{C}(31)$ & $114.73(19)$ \\
$\mathrm{N}(1)-\mathrm{Cu}(1)-\mathrm{N}(4)$ & $115.76(14)$ & $\mathrm{C}(26)-\mathrm{Cu}(2)-\mathrm{N}(11)$ & $113.59(16)$ \\
$\mathrm{N}(2)^{\prime}-\mathrm{Cu}(1)-\mathrm{N}(6)$ & $101.34(14)$ & $\mathrm{C}(31)-\mathrm{Cu}(2)-\mathrm{N}(11)$ & $104.95(16)$ \\
$\mathrm{N}(1)-\mathrm{Cu}(1)-\mathrm{N}(6)$ & $116.11(14)$ & $\mathrm{C}(26)-\mathrm{Cu}(2)-\mathrm{N}(9)$ & $106.35(17)$ \\
$\mathrm{N}(4)-\mathrm{Cu}(1)-\mathrm{N}(6)$ & $118.86(14)$ & $\mathrm{C}(31)-\mathrm{Cu}(2)-\mathrm{N}(9)$ & $111.99(17)$ \\
$\mathrm{N}(2)^{\prime}-\mathrm{Cu}(1)-\mathrm{N}(3)$ & $177.10(14)$ & $\mathrm{N}(11)-\mathrm{Cu}(2)-\mathrm{N}(9)$ & $104.91(14)$ \\
$\mathrm{N}(1)-\mathrm{Cu}(1)-\mathrm{N}(3)$ & $79.97(13)$ & & \\
\hline
\end{tabular}


The coordination sphere of each of the two $\mathrm{Cu}^{\mathrm{I}}$ ions consists of two side arm $\mathrm{N}$ atoms and two isocyanide co-ligands, resulting in a perfect tetrahedral geometry typical for $\mathrm{Cu}^{\mathrm{I}}$ ions. The observed distance between copper and the carbon from the isocyanide co-ligand $(\sim 1.93 \AA)$ is slightly longer than for previously described copper(I) systems stabilized by various isocyanide ligands. ${ }^{152,153,154,155}$

This is in good agreement with the features of the EPR spectrum obtained after addition of the isocyanide co-ligand: two copper(II) ions are strongly antiferromagnetically coupled via the pyrazole unit resulting in the formation of a bis( $\mu$-pyrazolato) dicopper(II) core. The two copper(I) ions in this complex are EPR-silent.

Taking this in account, it can be concluded that the yellow-green solution largely contains an EPR active, dinuclear mixed-valence $\mathrm{Cu}^{\mathrm{I}} \mathrm{Cu}^{\mathrm{II}}$ complex and also that addition of tert-butyl isocyanide induces a dimerisation of this species due to the coordination of the strong $\sigma$-donor isocyanide, resulting in a rearrangement of the mixed-valence $\mathrm{Cu}^{\mathrm{I}} \mathrm{Cu}^{\mathrm{II}}$ core and formation of a stable bis( $\mu$-pyrazolato) dicopper(II) motif.

\subsection{Coordination behaviour of the new ligand $\mathrm{HL}^{5}$ towards copper(I)}

The pyrazolate ligand $\mathbf{H L}^{\mathbf{5}}$, substituted in the 4-position by a phenyl group, was applied to study the coordination behaviour of the present new generation of ligands with pendant bis[(1-methylimidazole-2-yl)-methyl]amine side arms towards copper(I). Moreover, the information gained from the solid state structures and spectroscopic properties of these copper(I) complexes was considered very useful to determine the nature of various intermediate species formed upon reduction of the initial copper(II) species during oxidation and oxygenation reactions catalyzed by copper complexes with pyrazole-based ligands.

Reaction of $\mathbf{H L}^{\mathbf{5}}$ with one equivalent of $\mathrm{KO} t \mathrm{Bu}$ (for deprotonation of the pyrazole NH-unit) in $\mathrm{MeCN}$, followed by addition of two equivalents of $\left[\mathrm{Cu}(\mathrm{MeCN})_{4}\right]\left(\mathrm{PF}_{6}\right)$ to the reaction mixture, was performed under strict anaerobic conditions. After stirring of the light-yellow reaction mixture for $2 \mathrm{~h}$, one equivalent of tert-butyl isocyanide (per copper atom) was added. Characterization of the resultant crystalline material by IR spectroscopy revealed the presence of a band at $v_{\mathrm{CN}} 2164 \mathrm{~cm}^{-1}$, indicative of the presence of one isocyanide ligand per $\mathrm{Cu}^{\mathrm{I}}$ center. The single crystals of complex $\mathbf{1 0}$ that could be isolated after diffusion of $\mathrm{Et}_{2} \mathrm{O}$ into a $\mathrm{MeCN}$ 
solution proved to be of insufficient quality for a high-resolution X-ray crystallographic analysis and therefore a complete dataset could not be obtained. However, a rough estimation of intramolecular distances between key atoms (like $\mathrm{Cu}$ and $\mathrm{N}$ ) was possible on the basis of these measurements. The corresponding approximation of the molecular structure of $\mathbf{1 0}$ is shown in Figure 67.

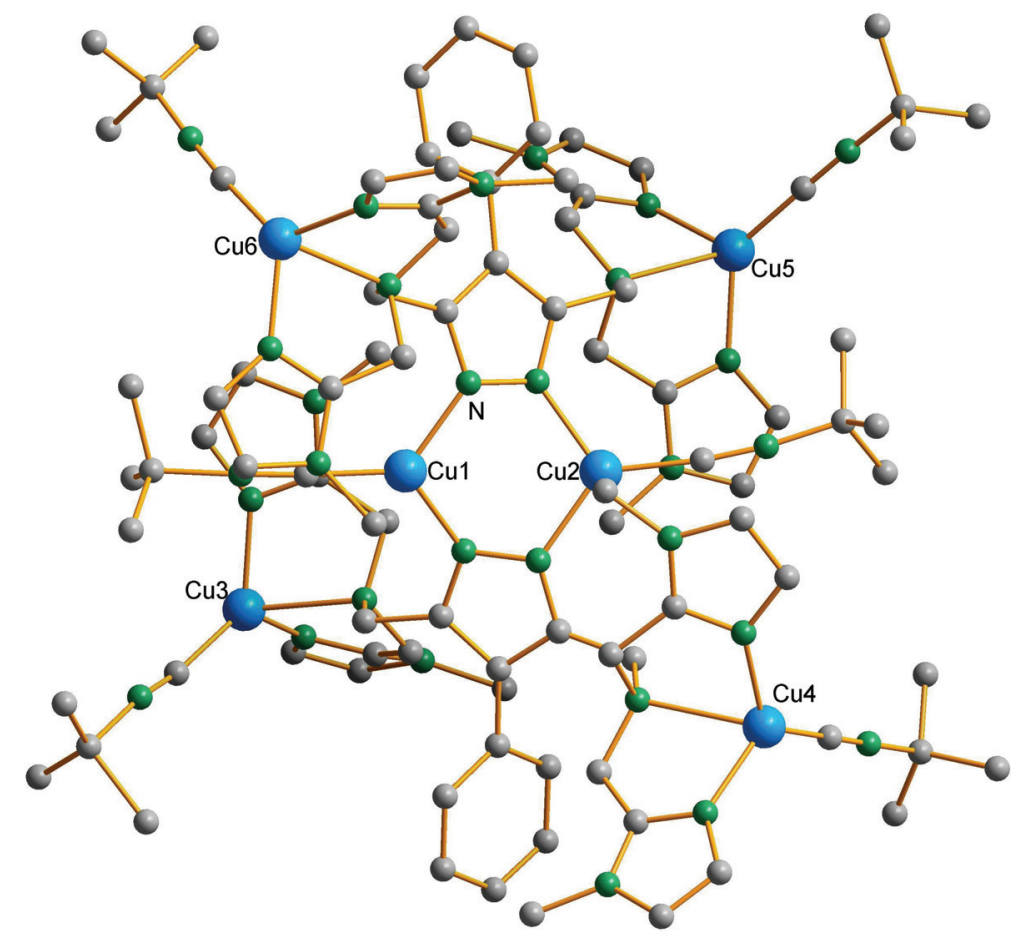

Figure 67. Molecular structure of the cation of $\mathbf{1 0 .}$

Instead of the expected dinuclear species, this complex exists as a hexanuclear species. Six copper atoms are coordinated to two ligands overall, with one additional isocyanide co-ligand per copper atom. The four outer copper(I) atoms that are nested in the dangling chelate side arms have distorted tetrahedral geometry. The two remaining copper(I) ions are bridged by the two pyrazolate cores of the respective ligands, which results in the formation of the central bis( $\mu$-pyrazolato) dicopper(I) core. Coordination of one additional isocyanide fragment per $\mathrm{Cu}$ leads to completion of the trigonal planar coordination environment for both central copper(I) atoms.

It is assumed that formation of this structural motif is promoted by coordination of the strong $\sigma$-donor isocyanide co-ligands, which induce a rearrangement of the putative dicopper(I) core that may have formed initially. It was clearly shown by means of ${ }^{1} \mathrm{H}$ NMR spectroscopy that 
the ratio between pyrazole ligand and tert-butyl isocyanide in the resulting copper(I) complex in solution is 1 to 3 (based on the integration ratio).

On these grounds it was proven that complex $\mathbf{1 0}$ exists in both, solid state and solution as hexanuclear species. These experiments show that pyrazole-based ligands substituted with bis[(1-methylimidazole-2-yl)-methyl]amine pockets can serve as a host for copper(I) and that such complexes can be stabilized in the presence of strong $\sigma$-donor co-ligands.

\subsection{Time-dependent concentration profiles of TMP, TMBB and TMSQ during catalysis with 5 a}

In order to learn about the formation of the $\mathrm{C}-\mathrm{C}$ coupled intermediate TMBB as well as of the oxidized product TMSQ over time under catalytic conditions, the concentration profiles of TMBB and TMP were measured by ${ }^{1} \mathrm{H}$ NMR spectroscopy, while the formation of TMSQ was followed by UV/vis spectroscopy.

\section{NMR monitoring:}

NMR monitoring was performed under aerobic conditions using the standard reaction conditions applied. To a $0.005 \mathrm{M}$ solution of dicopper(II) complex 5a, a $0.025 \mathrm{M}$ solution of TMP was added, resulting in a final volume of $64 \mathrm{~mL}$ of $\mathrm{MeCN} / \mathrm{CH}_{2} \mathrm{Cl}_{2}$ (4:6). 1,2,4,5Tetrabromobenzene was used as an internal standard to allow calculation of the yield of the formed TMBB and the conversion of TMP during $8 \mathrm{~h}$ reaction time. Aliquots of the reaction mixture $(4 \mathrm{~mL})$ were passed through a silica column using $\mathrm{CH}_{2} \mathrm{Cl}_{2}$ as eluent (final washing was performed using a mixture of $\mathrm{CH}_{2} \mathrm{Cl}_{2} / \mathrm{MeOH}$ 9:1) to quench the reaction by removal of the copper complex. After silica chromatography, solvents were removed in vacuo and ${ }^{1} \mathrm{H}$ NMR spectra of the resulting residues were measured in $\mathrm{CDCl}_{3}$. The concentrations of the formed TMBB as well as of the remaining TMP substrate were calculated from ${ }^{1} \mathrm{H}$ NMR data using the relative integrals of the signal at $6.76 \mathrm{ppm}$ for TMP and at $6.80 \mathrm{ppm}$ for TMBB (Figure 68 (a), (b)) and the signal for tetrabromobenzene at $7.80 \mathrm{ppm}$ as internal standard.

\section{UV/vis monitoring:}

UV/vis monitoring of the formation of the final product TMSQ was carried out using the same amounts of complex 5a and TMP as applied in the previous experiment. Aliquots of the 
reaction mixture $(1 \mathrm{~mL})$ were diluted with $\mathrm{CH}_{2} \mathrm{Cl}_{2}$, skipping the chromatographic procedure for removal of the copper complex, and UV/vis spectra were measured over a reaction time of $8 \mathrm{~h}$. The observed yield in TMSQ (absorption values at $440 \mathrm{~nm}\left(\varepsilon=96000 \mathrm{~L} \cdot \mathrm{mol}^{-1} \cdot \mathrm{cm}^{-1}\right.$ in $\mathrm{CH}_{2} \mathrm{Cl}_{2}$ ) were used for calculations) after $8 \mathrm{~h}$ was found to be $38 \%$ (Figure 68 (c)). In case of a longer reaction time ( $24 \mathrm{~h}$ and longer) the yield of the $\mathrm{C}-\mathrm{C}$ coupled product increased to 66 $\%$.

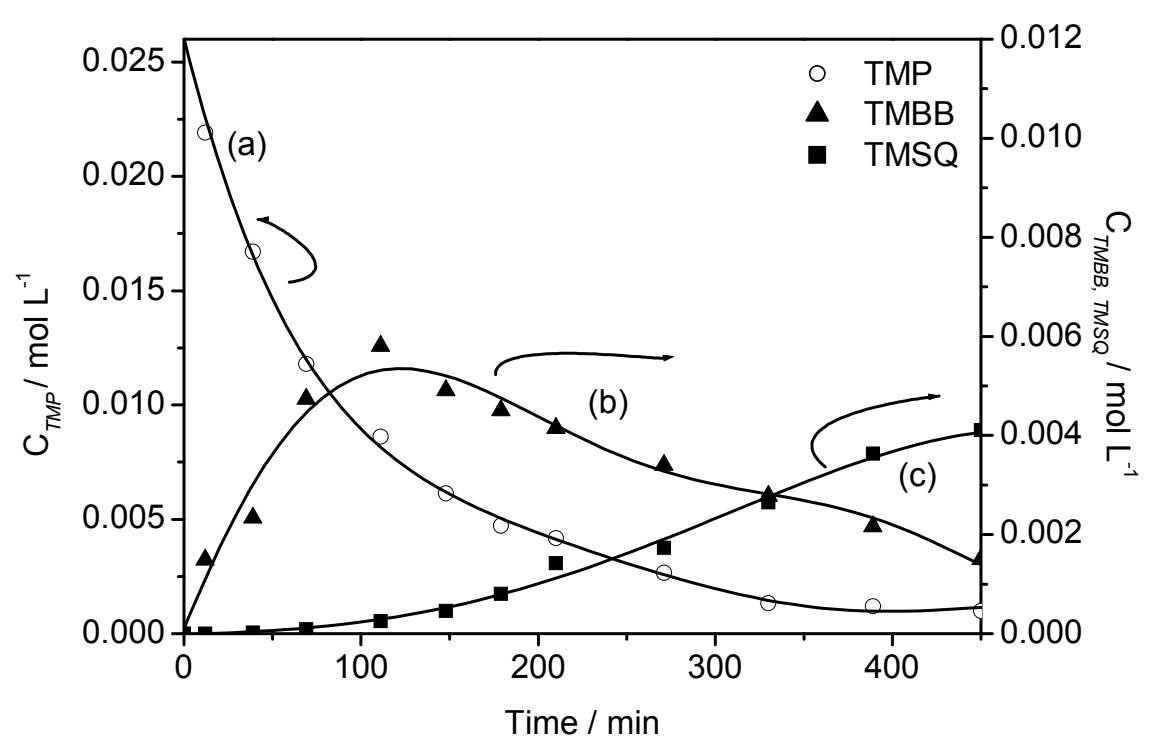

Figure 68. Time-dependent concentration profiles of TMP (a), TMBB (b) and TMSQ (c) (starting concentration of $\mathbf{5 a}=0.005 \mathrm{~mol} \cdot \mathrm{L}^{-1}$, concentration of TMP $=0.025 \mathrm{~mol} \cdot \mathrm{L}^{-1}$, solvent: $\left.\mathrm{MeCN} / \mathrm{CH}_{2} \mathrm{Cl}_{2} 4: 6 \mathrm{v} / \mathrm{v}\right)$.

An exponential decay was observed for the concentration profile of TMP, whereas the concentration of TMBB reached a maximum within $2 \mathrm{~h}$, concurrent with the rapid formation of TMSQ.

\subsection{Determination of the $\mathrm{O}_{2}$ derived by-product in the $\mathrm{C}-\mathrm{C}$ coupling reaction}

Oxidative C-C coupling of TMP to TMSQ catalyzed by 5a in the presence of dioxygen can result in either $\mathrm{H}_{2} \mathrm{O}_{2}$ or $\mathrm{H}_{2} \mathrm{O}$ as the reduction product (Scheme 36). These scenarios differ by the stoichiometry of substrate to oxidant $\left(\mathrm{O}_{2}\right)$. Determination of the reduction product is important for a full understanding of the mechanism behind this unusual $\mathrm{Cu}$-catalyzed reaction with TMP. 

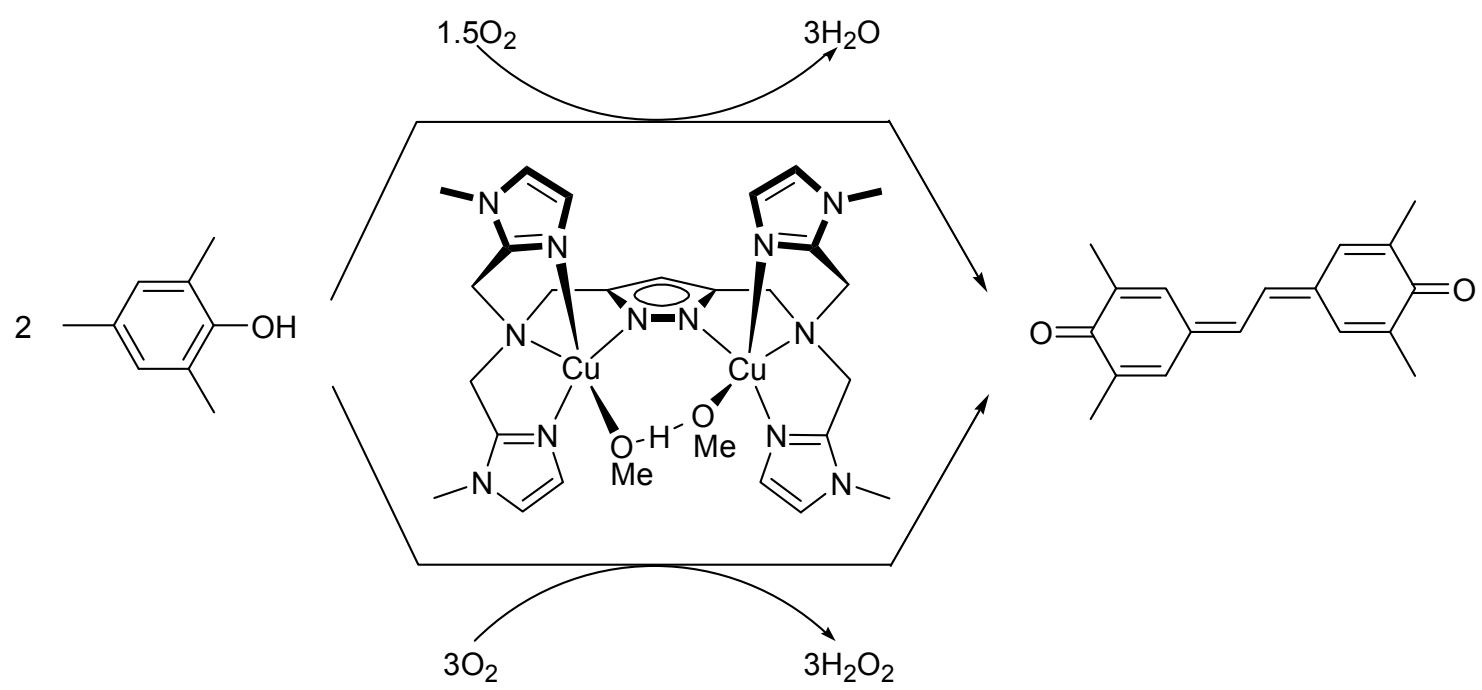

Scheme 36. Oxidative C-C coupling of TMP: $\mathrm{H}_{2} \mathrm{O}_{2}$ and $\mathrm{H}_{2} \mathrm{O}$ are possible reduction products.

The possible formation of $\mathrm{H}_{2} \mathrm{O}_{2}$ can be probed by some chemical tests that selectively detect the presence of this species, in the way described below. ${ }^{108}$

First the TMP substrate was oxidized to the product TMSQ in the presence of dicopper(II) catalyst 5a under aerobic conditions until the absorption at $\lambda_{\max } 440 \mathrm{~nm}$ had reached the arbitrary value of one in the UV/vis spectrum. The reaction was then quenched with an equimolar amount of a $0.005 \mathrm{M} \mathrm{H}_{2} \mathrm{SO}_{4}$ solution and the product TMSQ as well as residual TMP were removed by extraction with $\mathrm{CH}_{2} \mathrm{Cl}_{2}$. To $5 \mathrm{ml}$ of the remaining solution was added $2 \mathrm{ml}$ of $\mathrm{H}_{2} \mathrm{O}$ in the reference cell. In the sample cell $2 \mathrm{ml}$ of a $0.3 \mathrm{M} \mathrm{KI}$ solution and catalytic amounts of lactoperoxidase - for specific acceleration of the oxidation of $\mathrm{I}^{-}$to $\mathrm{I}_{3}^{-}$- were added. The development of the absorption band at $\lambda_{\max } 353\left(\varepsilon=26000 \mathrm{~L} \mathrm{~mol}^{-1} \mathrm{~cm}^{1}\right)$ for the resulting $\mathrm{I}_{3}{ }^{-}$species, which can only be formed in the presence of $\mathrm{H}_{2} \mathrm{O}_{2}$, was followed by $\mathrm{UV} / \mathrm{vis}$ spectroscopy. The blank experiment, without substrate present, was performed using the same procedure. Additionally, the possible formation of $\mathrm{H}_{2} \mathrm{O}_{2}$ as the reduction product during the C-C coupling was checked by using test strips from Merck.

Both tests applied were clearly negative, which convincingly shows that during the oxidative C-C coupling of TMP to TMSQ $\mathrm{H}_{2} \mathrm{O}_{2}$ is not formed. This means that water is formed as the reduction product, implying that 1.5 molar equivalents of $\mathrm{O}_{2}$ is needed per molecule of TMSQ formed. 


\section{$8 \quad$ Adducts with model substrate molecules}

Coordination of the TMP substrate within the bimetallic pocket of the dicopper core of 5a prior to oxidative $\mathrm{C}-\mathrm{C}$ coupling occurs under both aerobic and anaerobic conditions, as was shown by means of UV/vis spectroscopy (i.e. the Job experiment), in particular by the observation of a phenolate-to-copper CT band at a $\lambda_{\max }$ of $495 \mathrm{~nm}$. Resonance Raman spectroscopy also provided evidence for the formation of the dicopper(II)-phenolate species upon coordination of TMP.

In order to gain structural insight in the possible coordination behaviour of TMP towards copper catalyst 5a, a selected range of phenolic and diphenolic substrates (as models for TMBB), all of have redox potentials that prevent any $\mathrm{Cu}$-mediated reactivity, was studied. The interaction between these model substrates and 5a in solution is investigated spectroscopically and molecular structures of dicopper-phenolate complexes with PFP and 4AP as model substrates are discussed.

\subsection{Copper(II)-phenolate complexes}

Recently, considerable emphasis has been placed on the detailed study of the properties of $\mathrm{Cu}^{\mathrm{II}}$-phenolate complexes, because of their postulated involvement in a range of biological and catalytic processes. Thus, $\mathrm{Cu}^{\mathrm{II}}$-phenolate units have been proposed as intermediates in the catalytic cycles of metalloenzymes (e.g. galactose oxidase, ${ }^{156}$ tyrosinase $^{157}$ ) as well as in synthetic catalysis (e.g. alcohol oxidation, ${ }^{158}$ phenol polymerization ${ }^{159}$ ). ${ }^{160}$ The known $\mathrm{Cu}^{\mathrm{II}}$ phenolate complexes exhibit coordination numbers ranging from 4 to 6 , as is typical for the coordination chemistry of $\mathrm{Cu}^{\mathrm{II}}$. In addition, the phenolates in most of these compounds usually are incorporated as part of multidentate ligand systems; complexes with simple, exogenous phenolate ligands are less common. ${ }^{161}$

Several research groups have used ligands that have bis(pyridyl)alkylamines linked to a single phenol as a common design feature (Figure 69 (a)). These ligands represent a class of compounds where the phenol unit is incorporated into multidentate ligand systems. 


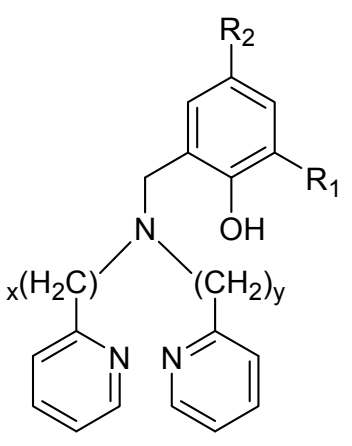

(a)

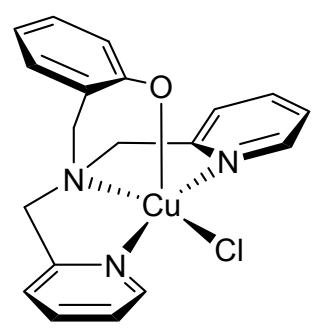

(b)

Figure 69. (a) General representation of the bis(pyridyl)alkylamine ligand framework $\mathrm{HL}^{\mathrm{Ph}}$ ( $\mathrm{x}, \mathrm{y}=1$ or $2, \mathrm{R}_{1}, \mathrm{R}_{2}=$ alkyl substituents); (b) schematic representation of the structure of the $\mathrm{Cu}^{\mathrm{II}}$-phenolate complex with $\mathrm{x}=\mathrm{y}=1, \mathrm{R}_{1}=\mathrm{R}_{2}=\mathrm{H}$.

This basic tripodal ligand design has been varied by adjusting the length of the alkyl chain linking the pyridine and tertiary amine donors ( $\mathrm{x}$ and $\mathrm{y}$ ), as well as by changing the phenol ring substituents $\left(\mathrm{R}_{1}\right.$ and $\left.\mathrm{R}_{2}\right)$. Numerous mononuclear complexes of the general formula $\left[\mathrm{L}^{\mathrm{Ph}} \mathrm{CuX}\right]\left(\mathrm{X}\right.$ is a monoanionic donor ligand, such as $\mathrm{Cl}^{-}$or $\mathrm{CH}_{3} \mathrm{CO}_{2}^{-} ; \mathrm{L}^{\mathrm{Ph}}$ is a phenolatecontaining bis(pyridyl)alkylamine ligand) have been prepared and some of them have been structurally characterized (molecular formula is depicted in Figure 69 (b)). Typical features of these compounds include a phenolate-to-copper(II) CT transition in their UV/vis spectrum $\left(\lambda_{\max } \sim 380-450 \mathrm{~nm}\right)$ as well as axial signals in their EPR spectrum. ${ }^{162}$

When the complex syntheses using bis(pyridyl)alkylamine/phenol ligands were performed in the absence of a strong monoanionic donor (e.g. with $\mathrm{Cu}\left(\mathrm{ClO}_{4}\right)_{2}, \mathrm{Cu}\left(\mathrm{BF}_{4}\right)_{2}$, or $\mathrm{CuSO}_{4}$ ), dimeric complexes of the formulation $[\mathrm{LCu}]_{2}{ }^{2+}$ were isolated. ${ }^{162 \mathrm{~d}, \mathrm{e}, 163} \mathrm{~A}$ representative structure of one such dimeric complex is shown in Figure 70.

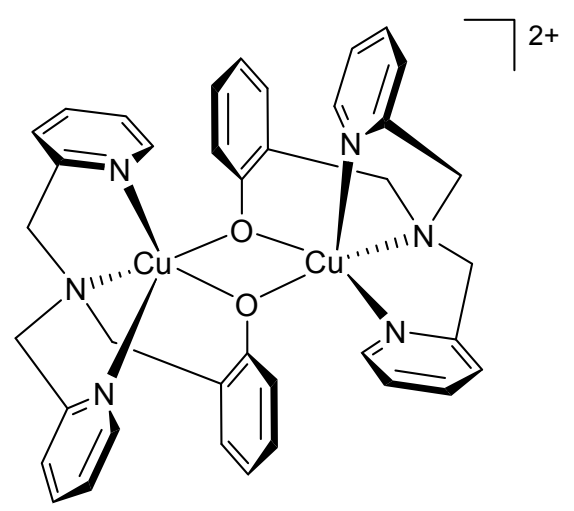

Figure 70. Schematic representation of a dimeric copper(II) complex with a phenolatederived multidentate ligand. 
All structurally characterized complexes are similar to the depicted one and show two $\mathrm{Cu}^{\mathrm{II}}$ ions in square pyramidal geometry, with slight trigonal bipyramidal distortion. The metal ions are bridged by two phenolates, one from each ligand. Like the monomeric complexes discussed above, the UV/vis spectra of the dimeric complexes exhibit a phenolate-tocopper(II) CT band (around $440 \mathrm{~nm}$ ) as well as $d-d$ transitions at a lower energy (around 630 $\mathrm{nm})$.

A recent example of a complex with a simple, exogenous phenolate ligand incorporated into a $\mathrm{Cu}$-complex was reported by Tolman and co-workers (Figure 71). ${ }^{132}$ Dark green, threecoordinate $\beta$-diketiminate-containing copper(II)-phenolate complexes were spectroscopically characterized and - in case of $\mathrm{R}=\mathrm{H}$ and $\mathrm{R}_{1}=\mathrm{OMe}$ in the para-position of the phenol ring the molecular structure was solved by X-ray crystallography.

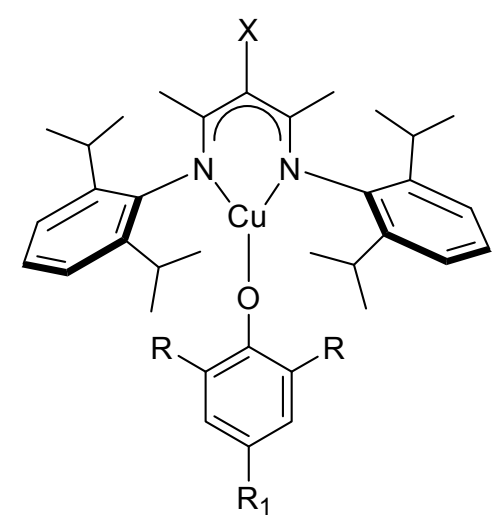

Figure 71. Schematic representation of the general structure of three-coordinate copper(II)phenolate complexes with $\beta$-diketiminate ligands.

The UV/vis spectrum of the complex with coordinated 4-tert-butylphenol showed intense $(\varepsilon \sim$ 1000-3000 $\mathrm{L} \mathrm{mol}^{-1} \mathrm{~cm}^{-1}$ ) absorption bands at $\lambda_{\max } \sim 450,625$ and $700 \mathrm{~nm}$ and on the basis of the resonance Raman experiments it was suggested that the absorption at $\lambda_{\max } \sim 625 \mathrm{~nm}$ possessed at least partial phenolate-to-copper(II) LMCT character. The Raman spectrum of a $\mathrm{CH}_{2} \mathrm{Cl}_{2}$ solution of the mononuclear copper(II) complex with 4-tert-butylphenolate ligand was measured using $633 \mathrm{~nm}$ laser excitation. The set of multiple features found between 1100 and $1600 \mathrm{~cm}^{-1}$ are typical of metal-phenolate complexes and metal-tyrosinase sites in proteins, ${ }^{164}$ with those at $1174,1270,1500$, and $1598 \mathrm{~cm}^{-1}$ being diagnostic for coordinated phenolates that have a substituent at the para-position. ${ }^{147,165}$ The bands at 1174 and $1270 \mathrm{~cm}^{-1}$ were assigned as $\mathrm{C}-\mathrm{H}$ bending and $\mathrm{C}-\mathrm{O}$ stretching modes, respectively, and those at 1500 and 
$1598 \mathrm{~cm}^{-1}$ were attributed to ring $\mathrm{C}-\mathrm{C}$ stretching vibrations for the coordinated phenolate ligand.

In this light, elucidation of the phenol binding within the dicopper core of 5a is of great interest not only in the frame of the present work but also with a general sense to investigate the behaviour of phenolic substrates towards dinuclear copper species.

\subsection{Characterization of the adducts with 4-hydroxybenzamide and pentafluorophenol}

With the aim to learn about the formation and structure of possible intermediates in the catalytic oxidative $\mathrm{C}-\mathrm{C}$ coupling reactions studied, the coordination chemistry of dicopper complex 5a towards phenolic substrates that can not display any $\mathrm{Cu}$-mediated reactivity was investigated. Pentafluorophenol (PFP) and 4-hydroxybenzamide (4-AP) were applied as model substrates to study the binding of the phenol within the bimetallic pocket of 5a. Reedijk and co-workers have shown that PFP can be used as an effective instrument to study the behaviour of phenolic substrates towards a copper catalyst. Thus, their copper catalyst based on the neocuproine ligand was shown to form a mononuclear copper complex, and formation of the presumably active dinuclear copper species during the catalysis was proven by using PFP as model substrate. ${ }^{99}$ Trapping of the active species was performed in the presence of base, which served as the second co-ligand to form a self-assembled 1-phenoxo-1methoxo-bridged dinuclear copper species with a mononucleating non-bridging ligand, namely neocuproine (see Figure 14 in section 3.3). The two copper(II) ions in the resulting complex are in square pyramidal environments and both the PFP and the methoxide are coordinated in a bridging mode to both copper ions. This type of coordination is governed by the possibility of two mononuclear copper species to assemble through the interaction of bridging co-ligands, whereby the $\mathrm{Cu} \cdots \mathrm{Cu}$ distance is determined solely by the phenolate and methoxide ligands. Therefore, the two copper ions are only $3.14 \AA$ apart.

Taking in account the completely different nature of the present dicopper(II) catalytic system, where two copper ions are already bridged by a pyrazole unit that features short $N, N$-bis[2-(1methylimidazolyl)methyl]amine side arms, the PFP substrate, which is inactive in any $\mathrm{Cu}$ mediated oxidation reactions, can possibly show a new coordination mode within the bimetallic pocket of $\mathbf{5 a}$. 
It was clearly shown (see section 7.5.4) that the presence of strong electron-withdrawing groups (e.g. $\mathrm{F}$ or $\mathrm{CONH}_{2}$ ) on the phenol shifts the redox potentials and renders the phenolic substrates inert towards any kind of oxidation reactions. The perfect triangular shape of the corresponding Job plots, particularly with PFP as a substrate, shows that the formed dicopperpentafluorophenolate complex exhibits significant stability and dissociation of the phenolate species does not occur to any significant extent in solution. Moreover, stable copperphenolate complexes with these phenols can be formed as a result of the coordination of only one substrate molecule.

The crystallization attempts were performed in the presence of dioxygen using a tenfold excess of pentafluorophenol relative to dicopper complex 5a. Yellow-green single crystals of copper-phenolate adduct 11, suitable for X-ray crystallographic analysis, were formed after layering the reaction mixture with $\mathrm{Et}_{2} \mathrm{O}$ overnight (Figure 72).

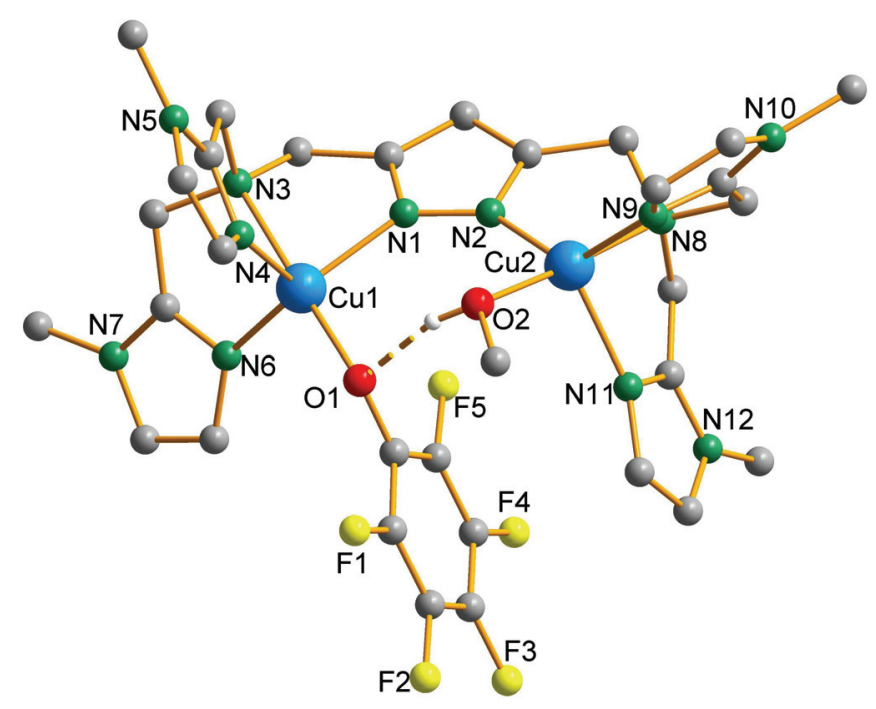

Figure 72. Molecular structure of the cation of $\mathbf{1 1 .}$

Table 17. Selected intramolecular distances $(\AA)$ and angles $\left(^{\circ}\right)$ for complex 11.

\begin{tabular}{llll}
\hline \multicolumn{3}{c}{ Distances } \\
\hline $\mathrm{Cu}(1)-\mathrm{O}(1)$ & $1.938(5)$ & $\mathrm{Cu}(2)-\mathrm{N}(2)$ & $1.970(5)$ \\
$\mathrm{Cu}(1)-\mathrm{N}(6)$ & $2.004(5)$ & $\mathrm{Cu}(2)-\mathrm{N}(9)$ & $2.025(5)$ \\
$\mathrm{Cu}(1)-\mathrm{N}(1)$ & $2.015(5)$ & $\mathrm{Cu}(2)-\mathrm{N}(8)$ & $2.088(5)$ \\
$\mathrm{Cu}(1)-\mathrm{N}(4)$ & $2.100(6)$ & $\mathrm{Cu}(2)-\mathrm{N}(11)$ & $2.147(5)$ \\
$\mathrm{Cu}(1)-\mathrm{N}(3)$ & $2.104(5)$ & $\mathrm{Cu}(1) \cdots \mathrm{Cu}(2)$ & $4.309(1)$
\end{tabular}




\begin{tabular}{llll}
$\mathrm{Cu}(2)-\mathrm{O}(2)$ & $1.960(5)$ & \\
\hline & \multicolumn{3}{c}{ Angles } \\
\hline $\mathrm{O}(1)-\mathrm{Cu}(1)-\mathrm{N}(6)$ & $101.7(2)$ & $\mathrm{O}(2)-\mathrm{Cu}(2)-\mathrm{N}(2)$ & $95.3(2)$ \\
$\mathrm{O}(1)-\mathrm{Cu}(1)-\mathrm{N}(1)$ & $99.4(2)$ & $\mathrm{O}(2)-\mathrm{Cu}(2)-\mathrm{N}(9)$ & $99.5(2)$ \\
$\mathrm{N}(6)-\mathrm{Cu}(1)-\mathrm{N}(1)$ & $127.8(2)$ & $\mathrm{N}(2)-\mathrm{Cu}(2)-\mathrm{N}(9)$ & $132.5(2)$ \\
$\mathrm{O}(1)-\mathrm{Cu}(1)-\mathrm{N}(4)$ & $95.6(2)$ & $\mathrm{O}(2)-\mathrm{Cu}(2)-\mathrm{N}(8)$ & $176.0(2)$ \\
$\mathrm{N}(6)-\mathrm{Cu}(1)-\mathrm{N}(4)$ & $116.7(2)$ & $\mathrm{N}(2)-\mathrm{Cu}(2)-\mathrm{N}(8)$ & $80.8(2)$ \\
$\mathrm{N}(1)-\mathrm{Cu}(1)-\mathrm{N}(4)$ & $107.8(2)$ & $\mathrm{N}(9)-\mathrm{Cu}(2)-\mathrm{N}(8)$ & $82.6(2)$ \\
$\mathrm{O}(1)-\mathrm{Cu}(1)-\mathrm{N}(3)$ & $175.3(2)$ & $\mathrm{O}(2)-\mathrm{Cu}(2)-\mathrm{N}(11)$ & $101.6(2)$ \\
$\mathrm{N}(6)-\mathrm{Cu}(1)-\mathrm{N}(3)$ & $82.0(2)$ & $\mathrm{N}(2)-\mathrm{Cu}(2)-\mathrm{N}(11)$ & $116.9(2)$ \\
$\mathrm{N}(1)-\mathrm{Cu}(1)-\mathrm{N}(3)$ & $80.3(2)$ & $\mathrm{N}(9)-\mathrm{Cu}(2)-\mathrm{N}(11)$ & $103.9(2)$ \\
$\mathrm{N}(4)-\mathrm{Cu}(1)-\mathrm{N}(3)$ & $80.1(2)$ & $\mathrm{N}(8)-\mathrm{Cu}(2)-\mathrm{N}(11)$ & $81.1(2)$ \\
\hline
\end{tabular}

A methanol-phenolate bridge between the two copper(II) centers was found in the molecular structure of the complex. Formation of such bridge proves that a methanolate-to-phenolate exchange has taken place at one $\mathrm{Cu}$ ion, effectively forming an $\mathrm{ArO} \cdots \mathrm{HOMe}$ bridge between the copper(II) centers. This closely matches the situation that is assumed to occur upon coordination of the active TMP substrate to 5a. The methanolate-for-phenolate exchange causes only slight changes in the coordination spheres of both copper ions (both metal ions are five-coordinated, $\tau_{\mathrm{Cu} 1}=0.80 ; \tau_{\mathrm{Cu} 2}=0.73$ ), which is reflected well in the UV/vis spectrum. For the resulting complex 11 the copper-based $d-d$ transition band was also observed at $\lambda_{\max }$ $989 \mathrm{~nm}$, and a charge-transfer band appeared at $\lambda_{\max }$ of $397 \mathrm{~nm}$ with an extinction coefficient that is typical for copper(II)-phenolate compounds ( $c a .1240 \mathrm{~L} \mathrm{~mol}^{-1} \mathrm{~cm}^{-1}$ ). Moreover, the molecular structure indicates an additional stabilization due to $\pi-\pi$ stacking interaction between the $\mathrm{C}_{6} \mathrm{~F}_{5}$-ring and one imidazole unit of the ligand (interplane distance $3.88 \AA$, angle $4.5^{\circ}$, Figure 73$)$. 


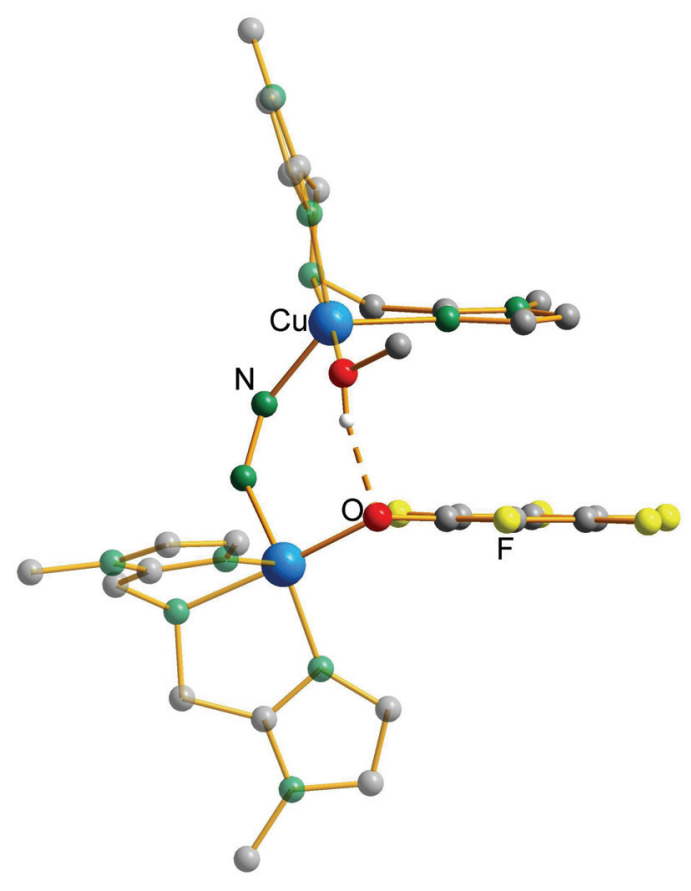

Figure 73. Side view of the molecular structure of the cation of 11; for clarity the carbon atoms of the pyrazole core are omitted.

As was supposed and intended when designing ligand $\mathbf{H L}^{4}$, the large separation between the copper(II) ions in complex $\mathbf{5 a}(d(\mathrm{Cu} \cdots \mathrm{Cu})=4.34 \AA$ ) $)$ is conditional for coordination of the PFP within the bimetallic core to only one of the copper ions, resulting in an almost unchanged copper-copper distance (4.31 $\AA$ ). The molecular structure of $\mathbf{1 1}$ also confirmed preliminary results from the Job plot experiment, where it was shown that coordination of only one PFP is optimal, thereby forming a very stable copper(II)-phenolate species.

In order to exclude any specific influence of substituents on the phenol ring on the interaction between the dicopper complex and the phenol substrate, 4-hydroxybenzamide was applied as a second model substrate to elucidate the phenol binding to the dicopper core of $\mathbf{5 a}$.

The slightly curved Job plot of 4-AP with dicopper complex 5a (see section 7.5.4) showed that the dicopper phenolate adduct exhibits moderate stability and dissociation of the phenolate species is still likely to occur to some degree in solution. From the performed Job plot experiment it was concluded that only one phenol molecule is able to coordinate to the dicopper core. Such an interaction in solution between 4-AP and dicopper complex 5a was unambiguously proven after analysis of single crystals that were obtained by slow diffusion of 
$\mathrm{Et}_{2} \mathrm{O}$ into a $\mathrm{MeCN}$ solution of $\mathbf{5 a}$ in the presence of excess of 4-AP. The molecular structure of the cation of $\mathbf{1 2}$ is depicted in Figure 74.

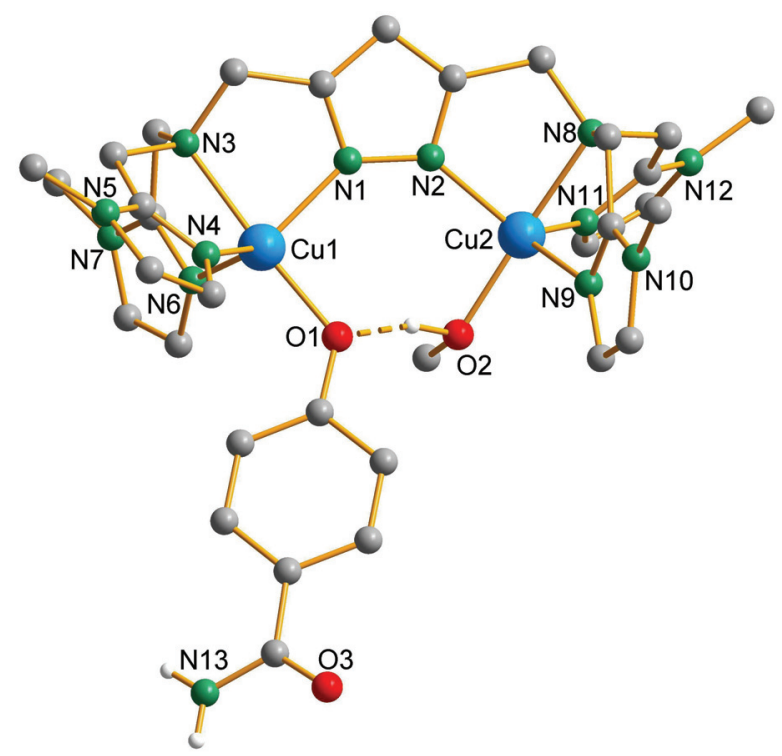

Figure 74. Molecular structure of the cation of 12.

Table 18. Selected intramolecular distances $(\AA)$ and angles $\left(^{\circ}\right)$ for complex 12.

\begin{tabular}{llll}
\hline \multicolumn{3}{c}{ Distances } \\
\hline $\mathrm{Cu}(1)-\mathrm{O}(1)$ & $1.919(7)$ & $\mathrm{Cu}(2)-\mathrm{N}(2)$ & $2.015(8)$ \\
$\mathrm{Cu}(1)-\mathrm{N}(1)$ & $2.024(8)$ & $\mathrm{Cu}(2)-\mathrm{N}(9)$ & $2.053(8)$ \\
$\mathrm{Cu}(1)-\mathrm{N}(4)$ & $2.040(8)$ & $\mathrm{Cu}(2)-\mathrm{N}(11)$ & $2.064(8)$ \\
$\mathrm{Cu}(1)-\mathrm{N}(6)$ & $2.087(8)$ & $\mathrm{Cu}(2)-\mathrm{N}(8)$ & $2.086(8)$ \\
$\mathrm{Cu}(1)-\mathrm{N}(3)$ & $2.114(8)$ & $\mathrm{Cu}(1) \cdots \mathrm{Cu}(2)$ & $4.387(2)$ \\
$\mathrm{Cu}(2)-\mathrm{O}(2)$ & $1.953(7)$ & & \\
\hline & & & \\
\hline $\mathrm{O}(1)-\mathrm{Cu}(1)-\mathrm{N}(1)$ & $95.7(3)$ & $\mathrm{O}(2)-\mathrm{Cu}(2)-\mathrm{N}(2)$ & $99.8(3)$ \\
$\mathrm{O}(1)-\mathrm{Cu}(1)-\mathrm{N}(4)$ & $99.8(3)$ & $\mathrm{O}(2)-\mathrm{Cu}(2)-\mathrm{N}(9)$ & $94.3(3)$ \\
$\mathrm{N}(1)-\mathrm{Cu}(1)-\mathrm{N}(4)$ & $120.0(3)$ & $\mathrm{N}(2)-\mathrm{Cu}(2)-\mathrm{N}(9)$ & $117.3(3)$ \\
$\mathrm{O}(1)-\mathrm{Cu}(1)-\mathrm{N}(6)$ & $102.4(3)$ & $\mathrm{O}(2)-\mathrm{Cu}(2)-\mathrm{N}(11)$ & $102.7(3)$ \\
$\mathrm{N}(1)-\mathrm{Cu}(1)-\mathrm{N}(6)$ & $115.0(3)$ & $\mathrm{N}(2)-\mathrm{Cu}(2)-\mathrm{N}(11)$ & $120.7(3)$ \\
$\mathrm{N}(4)-\mathrm{Cu}(1)-\mathrm{N}(6)$ & $117.3(3)$ & $\mathrm{N}(9)-\mathrm{Cu}(2)-\mathrm{N}(11)$ & $114.8(3)$ \\
$\mathrm{O}(1)-\mathrm{Cu}(1)-\mathrm{N}(3)$ & $176.0(3)$ & $\mathrm{O}(2)-\mathrm{Cu}(2)-\mathrm{N}(8)$ & $174.3(3)$ \\
$\mathrm{N}(1)-\mathrm{Cu}(1)-\mathrm{N}(3)$ & $80.6(3)$ & $\mathrm{N}(2)-\mathrm{Cu}(2)-\mathrm{N}(8)$ & $81.0(3)$
\end{tabular}




\begin{tabular}{llll}
$\mathrm{N}(4)-\mathrm{Cu}(1)-\mathrm{N}(3)$ & $80.9(3)$ & $\mathrm{N}(9)-\mathrm{Cu}(2)-\mathrm{N}(8)$ & $80.5(3)$ \\
$\mathrm{N}(6)-\mathrm{Cu}(1)-\mathrm{N}(3)$ & $80.6(3)$ & $\mathrm{N}(11)-\mathrm{Cu}(2)-\mathrm{N}(8)$ & $81.5(3)$ \\
\hline
\end{tabular}

The two copper ions in $\mathbf{1 2}$ reside within the adjacent $\left\{\mathrm{N}_{4}\right\}$ ligand compartments and are bridged by the pyrazolate core. Both metal ions are five-coordinate with almost perfect trigonal bipyramidal geometries $\left(\tau_{\mathrm{Cu} 1}=0.93 ; \tau_{\mathrm{Cu} 2}=0.90\right)$. The pyrazolate- $\mathrm{N}$ and the two imidazole- $\mathrm{N}$ atoms constitute the equatorial plains, while axial positions are occupied by the tertiary $\mathrm{N}$ atoms of the ligand backbone and by the $\mathrm{O}$ atoms of the $\mathrm{ArO} \cdots \mathrm{HOMe}$ moiety located within the bimetallic pocket $(d(\mathrm{Cu} \cdots \mathrm{Cu})=4.39 \AA)$. The slightly larger $\mathrm{Cu} \cdots \mathrm{Cu}$ distance found in $\mathbf{1 2}$ in comparison to both $\mathbf{5 a}$ and $\mathbf{1 1}$ could be explained by the absence of the $\pi-\pi$ stacking interaction between the $\mathrm{C}_{6} \mathrm{~F}_{5}$-ring and one imidazole unit of the ligand. Thus, in the molecular structure of the dicopper-phenolate complex 12, $\pi-\pi$ stacking interactions are absent. The coordinated 4-hydroxybenzamidate ligand is directed out from the bimetallic pocket and away from the imidazole rings. This presumably contributes to the lower binding affinity compared to the PFP adduct $\mathbf{1 1}$ and leads to a lengthening of the $\mathrm{Cu}^{\cdots} \mathrm{Cu}$ distance (Figure 75).

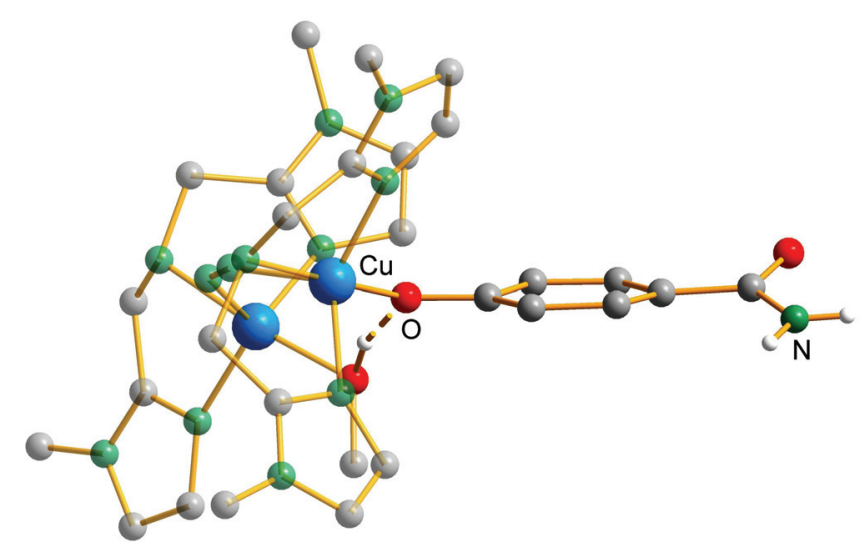

Figure 75. Side view of the molecular structure of the cation of 12; for clarity the carbon atoms of the pyrazole core are omitted.

As was observed for 11, formation of the dicopper-phenolate complex $\mathbf{1 2}$ did not result in any major changes in the $\mathrm{Cu} d-d$ region in the $\mathrm{UV} /$ vis spectrum, confirming that the coordination spheres of both copper ions are largely unchanged. The phenolate-to-copper CT transition band appeared at $446 \mathrm{~nm}$. 
Resonance Raman spectroscopy has been particularly useful for gaining insight into the structural and electronic properties of the dicopper(II)-phenolate complexes, in particular for complex 12. Laser excitation (488 nm) into the UV/vis CT transition band of $\mathbf{1 2}$ results in a resonance Raman spectrum that is very similar to those of other coordinated phenolates, ${ }^{132,165}$ with three dominant features at $1175 \mathrm{~cm}^{-1}$ ( $v_{9 \mathrm{a}}$, predominantly a C-H phenol stretching), 1282 $\mathrm{cm}^{-1}$ ( $v_{7 \mathrm{a}}, \mathrm{C}-\mathrm{O}$ stretching) and $1596 \mathrm{~cm}^{-1}$ ( $v_{8 \mathrm{a}}, \mathrm{C}_{\text {ortho }}-\mathrm{C}_{\text {meta }}$ ring stretching). The Raman spectrum of $\mathbf{1 2}$ is depicted in Figure 76.

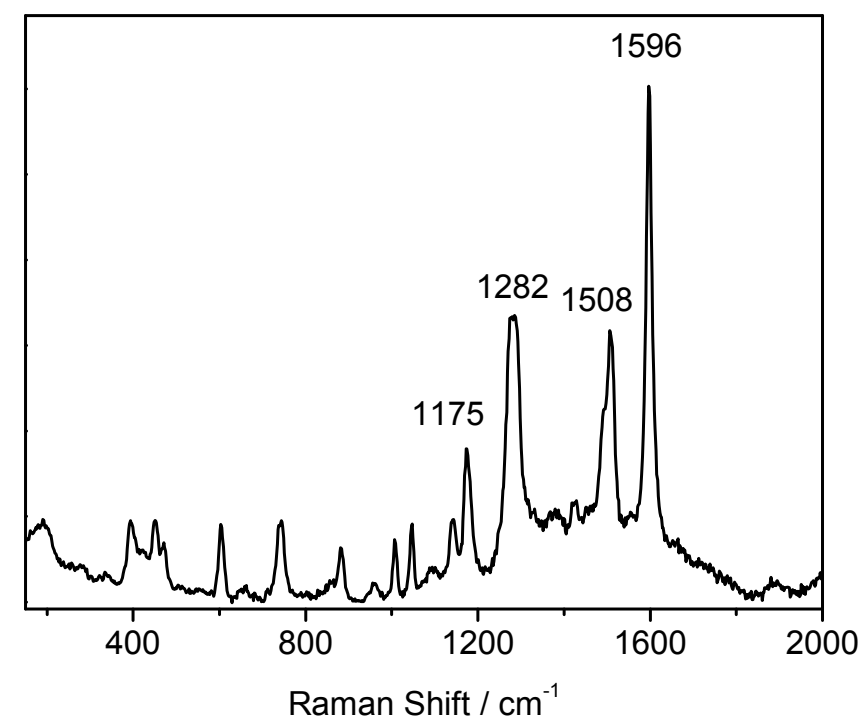

Figure 76. Solid state Raman spectrum of the dicopper(II)-phenolate complex 12.

Magnetic susceptibility data for a powdered sample of $\mathbf{1 2}$ were measured at two different magnetic fields $(2000 \mathrm{G}$ and $5000 \mathrm{G})$ in a temperature range from $2.0 \mathrm{~K}$ to $295 \mathrm{~K}$. No significant field dependence was observed. The temperature dependence of the magnetic susceptibility $\chi_{M}$ and of the product $\chi_{M} T$ for for $\mathbf{1 2}$ is depicted in Figure 77. 


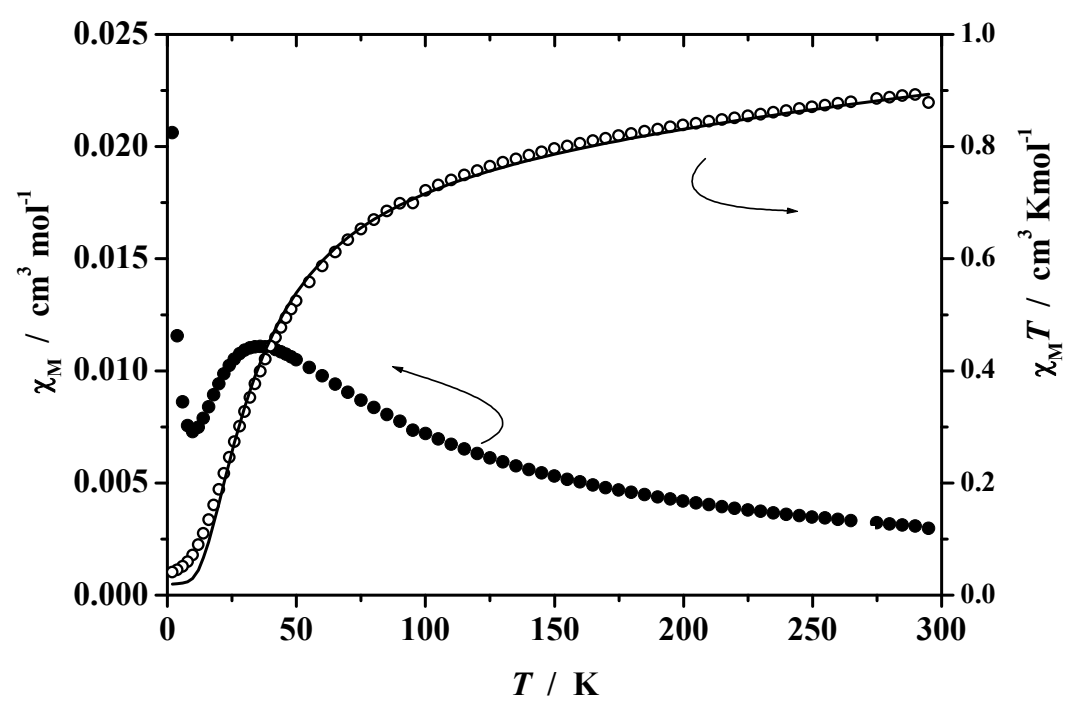

Figure 77. Plots of $\chi_{M}$ (solid circles) and $\chi_{M} T$ (open circles) versus temperature for 12 at $5000 \mathrm{G}$; the solid black line represents the calculated curve fits (see text).

The observed $\chi_{M} T$ value for 12 at $295 \mathrm{~K}$ is $0.89 \mathrm{~cm}^{3} \cdot \mathrm{K} \cdot \mathrm{mol}^{-1}\left(2.65 \mu_{\mathrm{B}}\right)$, which matches the value expected for two uncoupled copper(II) ions $\left(2.57 \mu_{\mathrm{B}}\right) \cdot \chi_{M} T$ decreases upon lowering the temperature and reaches zero below $15 \mathrm{~K}$, indicating an $S=0$ ground state, which is also evident from the broad maximum of the $\chi_{\mathrm{M}}$ versus $T$ curve that occurs around $40 \mathrm{~K}$. This is a typical signature for dinuclear copper complexes with relatively strong intramolecular antiferromagnetic coupling. The increase of $\chi_{M}$ at very low temperatures is presumably due to small amounts of paramagnetic impurities. Parameters according to the model described on page 65 are $J=-20.90 \mathrm{~cm}^{-1}, \mathrm{~g}=2.10, \rho \sim 5.0 \%, T I P=4.34 \cdot 10^{-4}$.

\subsection{4,4'-isopropylidene-bis(2,6-dimethylphenol) and 4,4'-(hexafluoroisopropylidene) diphenol as model substrates for TMBB}

Studies of the oxidative C-C coupling of TMP under anaerobic conditions showed that 4,4'dihydroxy-3,3',5,5'-tetramethylbibenzyl (TMBB) was the first intermediate formed (see section 7.6.3). For any further reaction (i.e. oxidation to TMSQ) to occur the presence of both dioxygen and copper complex 5a are required. Upon exposure of the yellow-green mixture, formed after storing the reaction mixture in the glove box for $48 \mathrm{~h}$, to molecular dioxygen or air, an intense charge-transfer band $\left(\lambda_{\max }=495 \mathrm{~nm}\right)$ again appeared in the UV/vis spectrum. In this case, no incubation period was observed, in contrast to the first oxidation step that 
leads to formation of TMBB, and instantaneous formation of TMSQ occurred. It was shown that when 5 equivalents of TMP were used relative to 5a, only one substrate molecule underwent coupling to yield TMBB in a stoichiometric reaction, while the remaining four equivalents did not react. Taking this in account, instantaneous formation of TMSQ after exposure of the yellow-green mixture to $\mathrm{O}_{2}$ can presumably take place only upon coordination of the formed TMBB intermediate within the dicopper core of 5a (Figure 78 (a)). This observation also justifies the conclusion that the $2 \mathrm{e}^{-}$oxidation of TMP to TMBB is the rate limiting step, while the further $4 \mathrm{e}^{-}$oxidation of TMBB to TMSQ occurs very rapidly.

Assuming that coordination of TMBB within the bimetallic pocket of the catalyst 5a, which was regenerated after reoxidation of the $\mathrm{Cu}^{\mathrm{I}} \mathrm{Cu}^{\mathrm{II}}$ species by dioxygen, actually takes place and is necessary for the subsequent reaction, 4,4'-isopropylidene-bis(2,6-dimethylphenol) $\left(\mathrm{HOCH}_{3} \mathrm{BP}\right)$ and $4,4^{\prime}$-(hexafluoroisopropylidene) diphenol $\left(\mathrm{HOCF}_{3} \mathrm{BP}\right)$ were applied as model substrates to study the binding of TMBB to $\mathbf{5 a}$ (Figure $78(\mathrm{~b}, \mathrm{c})$ ).

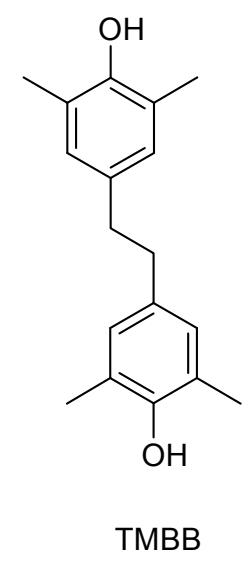

(a)

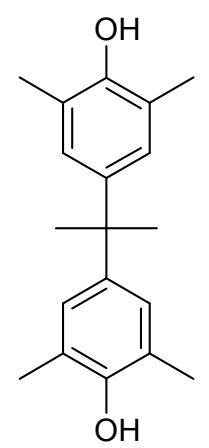

$\mathrm{HOCH}_{3} \mathrm{BP}$

(b)<smiles>Oc1ccc(C(c2ccc(O)cc2)(C(F)(F)F)C(F)(F)F)cc1</smiles>

$\mathrm{HOCF}_{3} \mathrm{BP}$

(c)

Figure 78. Intermediate species $\mathrm{TMBB}$ (a) and model substrates $\mathrm{HOCH}_{3} \mathrm{BP}$ (b) and $\mathrm{HOCF}_{3} \mathrm{BP}(\mathrm{c})$.

The model diphenols as well as TMBB contain two OH-groups as potential active units for binding to dicopper complex 5a. This might be the reason for the rapid formation of TMSQ upon exposure of the yellow-green solution to $\mathrm{O}_{2}$, i.e. either coordination of TMBB as a monodentate ligand to $\mathbf{5 a}$ or as a bridging ligand between two $\mathbf{5 a}$ species.

The absence of any possibility to build up a conjugated C-C system - as present in TMSQ gives rise to the inert behaviour of these two model substrates towards any kind of oxidation reactions. On the one hand, 4,4'-isopropylidenebis(2,6-dimethylphenol) $\left(\mathrm{HOCH}_{3} \mathrm{BP}\right)$ possesses very similar structural features as the active TMBB intermediate, with phenol rings 
that are substituted only by aliphatic $\mathrm{CH}_{3}$-groups in both ortho-positions. On the other hand, use of the second model substrate $\mathrm{HOCF}_{3} \mathrm{BP}$, which contains strong electron-withdrawing $\mathrm{CF}_{3}$-groups, will also allow studies on the binding to $\mathbf{5 a}$, while at the same time chances for the isolation of single crystals may be better, as observed with PFP.

In order to distinguish between the two proposed coordination modes of TMBB with 5a (see above) Job's method was applied as a useful instrument to determine the stoichiometry for the reaction occurring. Thus, in case of monodentate coordination of diphenols, the optimal value of $b$ (molar fraction in diphenol) should be equal to $\sim 0.5$, meaning the formation of a $1: 1$ complex of diphenol and 5a. The bridging coordination mode of the applied diphenols implies formation of a 1:2 complex of diphenol and 5a, which would lead to an expected value of 0.33 for $b$.

The Job plot of $\mathrm{HOCF}_{3} \mathrm{BP}$ with 5a was performed using the dilution technique (see section 7.5.3). The initial solution of dicopper complex 5a in $\mathrm{MeCN}$ was diluted further in a stepwise manner by addition of a solution of diphenol with a constant concentration. The UV/vis spectrum of the mixture was recorded after each dilution step (Figure 79).

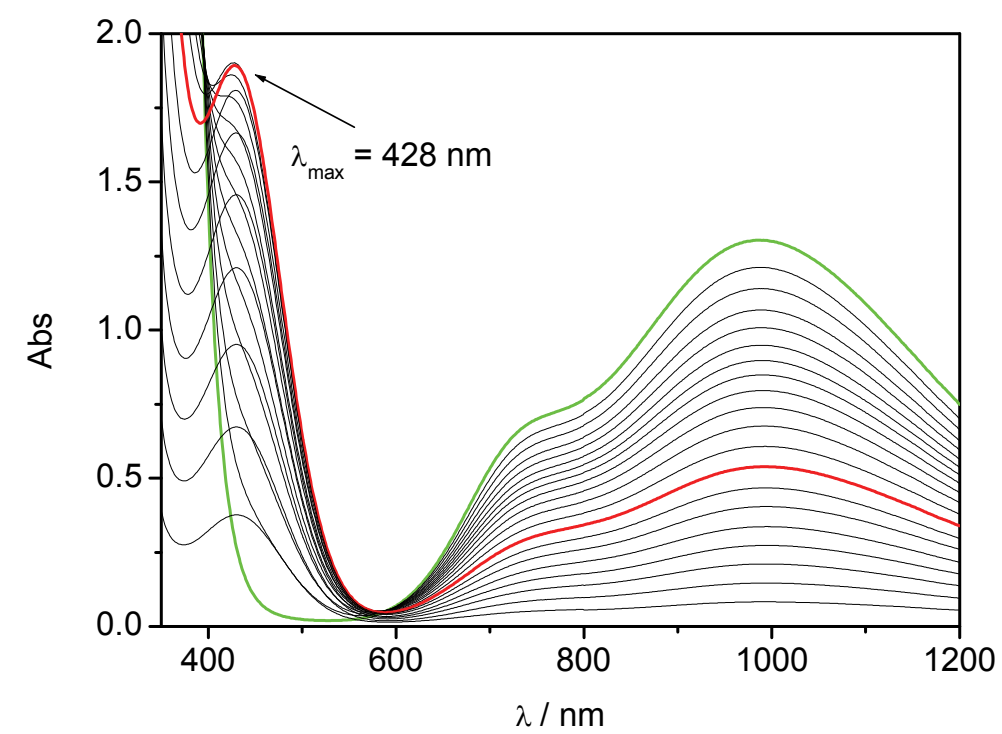

Figure 79. UV/vis spectra for the titration of $\mathbf{5 a}$ with $\mathrm{HOCF}_{3} \mathrm{BP}$ after each dilution step: green line (initial dicopper complex 5a); red line (solution of $\mathbf{5 a}$ and $\mathrm{HOCF}_{3} \mathrm{BP}$ with a maximum absorbance).

The corresponding absorption value of the LMCT band at $\lambda_{\max } 428 \mathrm{~nm}$ for each mixture was plotted versus the molar fraction in diphenol (b) (Figure 80). 


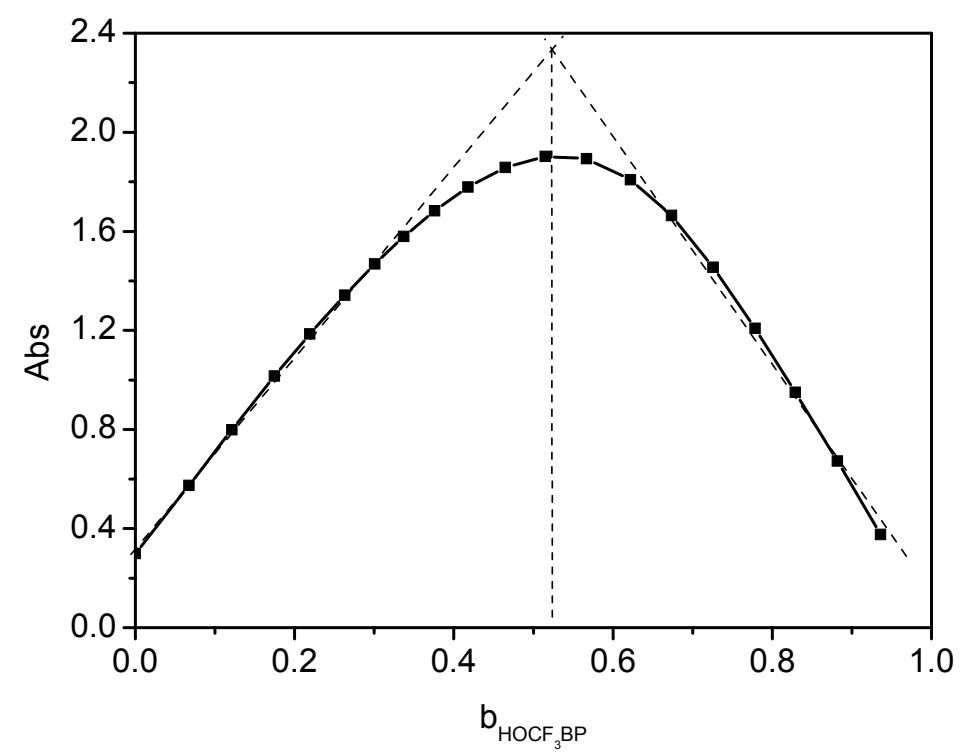

Figure 80. Job plot of $\mathrm{HOCF}_{3} \mathrm{BP}$ with dicopper complex 5a, $\lambda_{\max }=428 \mathrm{~nm}$.

From the obtained Job plot it can be concluded that formation of a dicopper-diphenolate species took place and moreover, that the formed species contained only one $\mathrm{HOCF}_{3} \mathrm{BP}(\mathrm{b} \sim$ 0.5) molecule per dicopper unit. The slightly curved plot shows that the dissociation of the diphenolate species is still likely to occur to some degree in solution.

It was not possible to perform the identical Job plot experiment with $\mathrm{HOCH}_{3} \mathrm{BP}$ as described above for $\mathrm{HOCF}_{3} \mathrm{BP}$ : addition of the substrate $\mathrm{HOCH}_{3} \mathrm{BP}$ to a green solution of $\mathbf{5 a}$ in $\mathrm{MeCN}$ initially led to the usual formation of a red coloured copper-phenolate species, but this proved to be only a short-lived complex, as a further unexpected colour change to deep green occurred within ten minutes. The same colour change was also observed, but at a significantly slower rate, for the red mixture of $\mathrm{HOCF}_{3} \mathrm{BP}$ with $\mathbf{5 a}$ obtained after the Job experiment, while this was subjected to a crystallization attempt. Slow diffusion of $\mathrm{Et}_{2} \mathrm{O}$ into the red mixture of $\mathrm{HOCF}_{3} \mathrm{BP}$ and $\mathbf{5 a}$ induced the formation of dark green crystals. Surprisingly, an unexpected tetranuclear copper complex was isolated (Figure 81). 


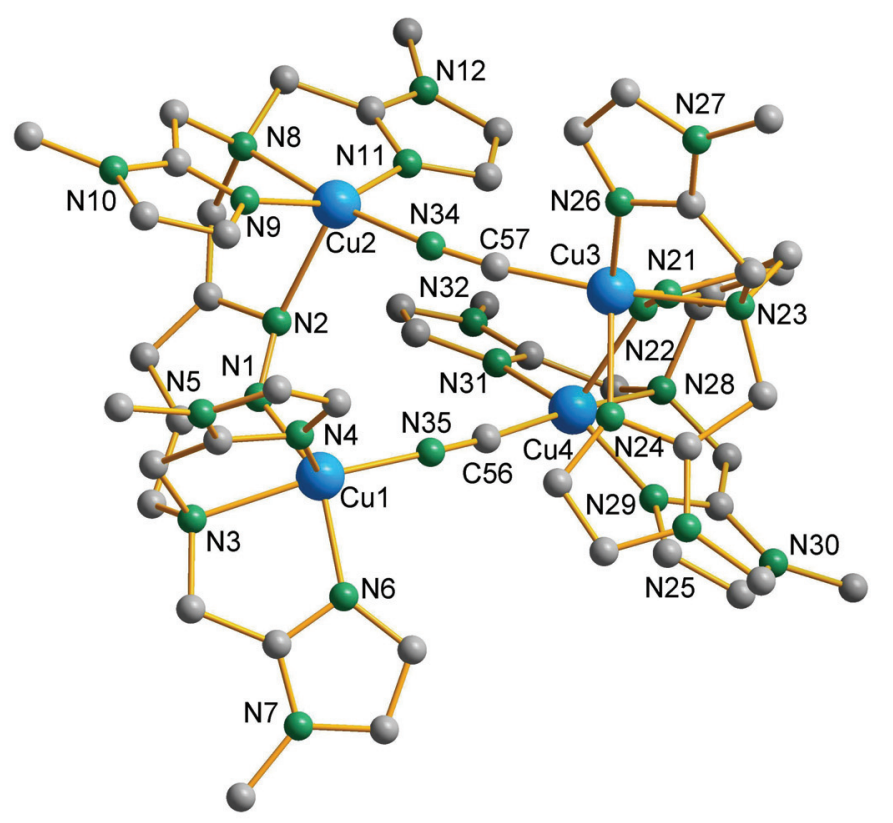

Figure 81. Molecular structure of the cation of tetranuclear complex 13.

Table 19. Selected intramolecular distances $(\AA)$ and angles $\left(^{\circ}\right)$ for complex 13.

\begin{tabular}{llll}
\hline \multicolumn{3}{c}{ Distances } \\
\hline $\mathrm{Cu}(1)-\mathrm{N}(1)$ & $1.952(2)$ & $\mathrm{Cu}(3)-\mathrm{N}(24)$ & $2.112(3)$ \\
$\mathrm{Cu}(1)-\mathrm{N}(35)$ & $1.969(3)$ & $\mathrm{Cu}(3)-\mathrm{N}(23)$ & $2.143(2)$ \\
$\mathrm{Cu}(1)-\mathrm{N}(6)$ & $1.987(3)$ & $\mathrm{Cu}(4)-\mathrm{C}(56)$ & $1.958(3)$ \\
$\mathrm{Cu}(1)-\mathrm{N}(3)$ & $2.178(2)$ & $\mathrm{Cu}(4)-\mathrm{N}(31)$ & $1.965(3)$ \\
$\mathrm{Cu}(1)-\mathrm{N}(4)$ & $2.189(2)$ & $\mathrm{Cu}(4)-\mathrm{N}(29)$ & $1.979(3)$ \\
$\mathrm{Cu}(2)-\mathrm{N}(11)$ & $1.958(2)$ & $\mathrm{Cu}(4)-\mathrm{N}(28)$ & $2.159(3)$ \\
$\mathrm{Cu}(2)-\mathrm{N}(9)$ & $1.970(2)$ & $\mathrm{Cu}(4)-\mathrm{N}(22)$ & $2.186(3)$ \\
$\mathrm{Cu}(2)-\mathrm{N}(34)$ & $1.982(3)$ & $\mathrm{Cu}(1) \cdots \mathrm{Cu}(2)$ & $4.4169(5)$ \\
$\mathrm{Cu}(2)-\mathrm{N}(8)$ & $2.164(2)$ & $\mathrm{Cu}(1) \cdots \mathrm{Cu}(3)$ & $5.6224(5)$ \\
$\mathrm{Cu}(2)-\mathrm{N}(2)$ & $2.257(2)$ & $\mathrm{Cu}(1) \cdots \mathrm{Cu}(4)$ & $5.0210(5)$ \\
$\mathrm{Cu}(3)-\mathrm{C}(57)$ & $1.957(3)$ & $\mathrm{Cu}(2) \cdots \mathrm{Cu}(3)$ & $5.0241(5)$ \\
$\mathrm{Cu}(3)-\mathrm{N}(21)$ & $2.015(2)$ & $\mathrm{Cu}(2) \cdots \mathrm{Cu}(4)$ & $5.3618(5)$ \\
$\mathrm{Cu}(3)-\mathrm{N}(26)$ & $2.021(3)$ & $\mathrm{Cu}(3) \cdots \mathrm{Cu}(4)$ & $4.4632(5)$ \\
\hline & & $\mathrm{Angles}$ & $\mathrm{C}(57)-\mathrm{Cu}(3)-\mathrm{N}(21)$ \\
\hline $\mathrm{N}(1)-\mathrm{Cu}(1)-\mathrm{N}(35)$ & $95.04(11)$ & $\mathrm{C}(57)-\mathrm{Cu}(3)-\mathrm{N}(26)$ & $96.70(11)$ \\
$\mathrm{N}(1)-\mathrm{Cu}(1)-\mathrm{N}(6)$ & $144.89(10)$ & $\mathrm{N}(21)-\mathrm{Cu}(3)-\mathrm{N}(26)$ & $128.28(13)$ \\
$\mathrm{N}(35)-\mathrm{Cu}(1)-\mathrm{N}(6)$ & $95.28(11)$ & &
\end{tabular}




$\begin{array}{llll}\mathrm{N}(1)-\mathrm{Cu}(1)-\mathrm{N}(3) & 79.18(10) & \mathrm{C}(57)-\mathrm{Cu}(3)-\mathrm{N}(24) & 105.46(11) \\ \mathrm{N}(35)-\mathrm{Cu}(1)-\mathrm{N}(3) & 163.18(10) & \mathrm{N}(21)-\mathrm{Cu}(3)-\mathrm{N}(24) & 105.63(10) \\ \mathrm{N}(6)-\mathrm{Cu}(1)-\mathrm{N}(3) & 81.34(10) & \mathrm{N}(26)-\mathrm{Cu}(3)-\mathrm{N}(24) & 115.86(12) \\ \mathrm{N}(1)-\mathrm{Cu}(1)-\mathrm{N}(4) & 106.12(10) & \mathrm{C}(57)-\mathrm{Cu}(3)-\mathrm{N}(23) & 174.86(11) \\ \mathrm{N}(35)-\mathrm{Cu}(1)-\mathrm{N}(4) & 117.41(10) & \mathrm{N}(21)-\mathrm{Cu}(3)-\mathrm{N}(23) & 78.27(10) \\ \mathrm{N}(6)-\mathrm{Cu}(1)-\mathrm{N}(4) & 98.59(10) & \mathrm{N}(26)-\mathrm{Cu}(3)-\mathrm{N}(23) & 80.21(10) \\ \mathrm{N}(3)-\mathrm{Cu}(1)-\mathrm{N}(4) & 79.41(9) & \mathrm{N}(24)-\mathrm{Cu}(3)-\mathrm{N}(23) & 79.62(10) \\ \mathrm{N}(11)-\mathrm{Cu}(2)-\mathrm{N}(9) & 156.45(10) & \mathrm{C}(56)-\mathrm{Cu}(4)-\mathrm{N}(31) & 96.84(11) \\ \mathrm{N}(11)-\mathrm{Cu}(2)-\mathrm{N}(34) & 94.93(10) & \mathrm{C}(56)-\mathrm{Cu}(4)-\mathrm{N}(29) & 97.01(12) \\ \mathrm{N}(9)-\mathrm{Cu}(2)-\mathrm{N}(34) & 101.65(10) & \mathrm{N}(31)-\mathrm{Cu}(4)-\mathrm{N}(29) & 148.13(11) \\ \mathrm{N}(11)-\mathrm{Cu}(2)-\mathrm{N}(8) & 79.95(10) & \mathrm{C}(56)-\mathrm{Cu}(4)-\mathrm{N}(28) & 172.94(11) \\ \mathrm{N}(9)-\mathrm{Cu}(2)-\mathrm{N}(8) & 82.05(10) & \mathrm{N}(31)-\mathrm{Cu}(4)-\mathrm{N}(28) & 80.67(10) \\ \mathrm{N}(34)-\mathrm{Cu}(2)-\mathrm{N}(8) & 173.26(11) & \mathrm{N}(29)-\mathrm{Cu}(4)-\mathrm{N}(28) & 82.10(11) \\ \mathrm{N}(11)-\mathrm{Cu}(2)-\mathrm{N}(2) & 104.18(9) & \mathrm{C}(56)-\mathrm{Cu}(4)-\mathrm{N}(22) & 104.74(11) \\ \mathrm{N}(9)-\mathrm{Cu}(2)-\mathrm{N}(2) & 87.85(9) & \mathrm{N}(31)-\mathrm{Cu}(4)-\mathrm{N}(22) & 108.57(10) \\ \mathrm{N}(34)-\mathrm{Cu}(2)-\mathrm{N}(2) & 104.42(10) & \mathrm{N}(29)-\mathrm{Cu}(4)-\mathrm{N}(22) & 95.39(11) \\ \mathrm{N}(8)-\mathrm{Cu}(2)-\mathrm{N}(2) & 81.23(9) & \mathrm{N}(28)-\mathrm{Cu}(4)-\mathrm{N}(22) & 82.33(10)\end{array}$

The same structural motif as for tetranuclear complex $\mathbf{8}$ is observed for $\mathbf{1 3}$ with the only difference being the bridging ligand. Thus, in complex 13 two dicopper units are bridged by $\mu$-CN ligands, while in tetranuclear complex 8 two $\mu-\mathrm{OH}$ groups are spanning two dicopper units (see section 5.5). The $\mathrm{Cu}-\mathrm{C}_{\mathrm{CN}}(\sim 1.96 \AA)$ and $\mathrm{Cu}-\mathrm{N}_{\mathrm{CN}}(\sim 1.97 \AA)$ atom distances are in agreement with a related end-on cyano-bridged dicopper complex reported in the literature. ${ }^{166}$ All four copper atoms are ligated by four $\mathrm{N}$ atoms, i.e. one from the pyrazolate-unit, two from the imidazoles in the side arm and by the tertiary $\mathrm{N}$ atom of the ligand backbone. The coordination environment of $\mathrm{Cu} 3$ and $\mathrm{Cu} 4$ is completed by $\mathrm{C}$ atoms from the bridging $\mathrm{CN}$ groups, while the remaining $\mathrm{Cu} 1$ and $\mathrm{Cu} 2$ ions are coordinated to the $\mathrm{N}$ atom from the same $\mathrm{CN}$-groups. In marked contrast to copper complex $\mathbf{8}$, this ligation results in a distorted trigonal bipyramidal geometry only for $\mathrm{Cu} 3(\tau=0.76)$ while the other copper atoms are in a distorted square pyramidal coordination environment $(\mathrm{Cu} 1: \tau=0.31 ; \mathrm{Cu} 2: \tau=0.28$ and $\mathrm{Cu} 4: \tau$ $=0.40$ ). These structural features were perfectly reproduced in the corresponding $\mathrm{UV} / \mathrm{vis}$ spectrum in solution, as $d-d$ transition bands were observed for a trigonal bipyramidal copper ion at $\lambda_{\max }$ of $879 \mathrm{~nm}$ as well as for copper ions in a square pyramidal environment at $\lambda_{\max }$ of $671 \mathrm{~nm}$. 
The ESI spectrum of a MeCN solution of $\mathbf{1 3}$ did not reveal the presence of this particular tetranuclear species in solution. However, FAB mass spectrometry allowed the detection of complex 13 with signals at $627,728,754$ and $1508 \mathrm{~m} / \mathrm{z}$, which correspond to $\left[\mathrm{Cu}_{2} \mathrm{~L}^{4}\right]^{+}$, $\left[\mathrm{Cu}_{2} \mathrm{~L}^{4}\left(\mathrm{ClO}_{4}\right)\right]^{+},\left[\mathrm{Cu}_{2} \mathrm{~L}^{4}(\mathrm{CN})\left(\mathrm{ClO}_{4}\right)\right]^{+}$and $\left[\mathrm{Cu}_{4} \mathrm{~L}_{2}^{4}(\mathrm{CN})_{2}\left(\mathrm{ClO}_{4}\right)_{2}\right]^{+}$, respectively.

In the IR spectrum of $\mathbf{1 3}$ a characteristic $v_{\mathrm{CN}}$ stretch for end-on bridging cyano-fragments was observed at $2173 \mathrm{~cm}^{-1}$, which is very close to the value found for the related tetranuclear system reported by Karlin and co-workers. ${ }^{166}$ This reported tetranuclear copper complex was formed from a dicopper(II)-hydroperoxo species through nucleophilic attack on the nitrile solvent used for reaction. In this light, the source of the $\mathrm{CN}$-group incorporated into the structure of complex $\mathbf{1 3}$ is most likely acetonitrile used for reaction. Unfortunately, the mechanistic aspects and the prerequisites for the observed $\mathrm{MeCN}$ oxidation are not clear till now, especially since in the present case the copper ions in the initial complex 5a are already in the oxidation state +2 , whereas the complex used by Karlin starts out as a copper(I) species that activates dioxygen.

\subsection{A tetrachlorocatechol-adduct of dicopper(II) complex 5a}

Oxidation of catechols to the corresponding quinones is catalyzed by the type 3 copper center in the enzyme catechol oxidase. Nowadays, many studies are focussed on investigating the mechanism behind this catalytic reaction. A plethora of mono- and dinuclear copper complexes have been synthesized and investigated as biomimetic catalysts for the catechol oxidation, ${ }^{65,11}$ in most cases using 3,5-di-tert-butylcatechol (DTBC) as the model substrate. $^{72,75,78 a, 108,167,168,169,170}$ The bulky tert-butyl groups of DTBC prevent unwanted side reactions such as ring opening or polymerization of the resulting quinone. Depending on the particular $\mathrm{Cu}$ catalyst used, either $\mathrm{H}_{2} \mathrm{O}_{2}$ or $\mathrm{H}_{2} \mathrm{O}$ may result as the reduction product (Scheme $37)$. 


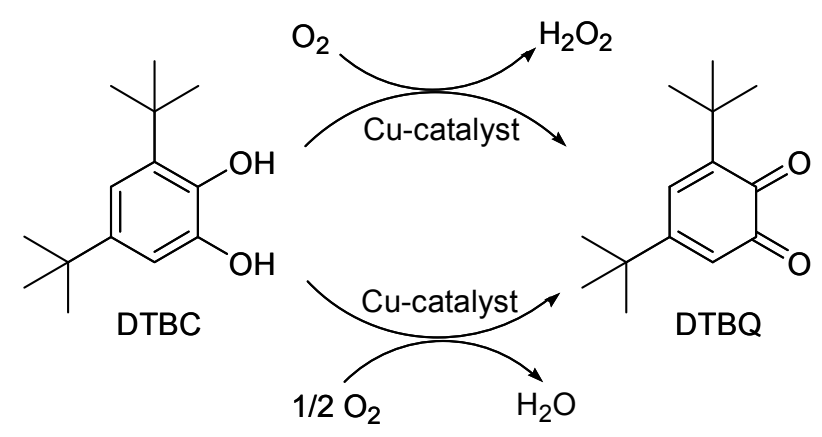

Scheme 37. Possible pathways for catechol oxidation catalyzed by a copper-based catalyst.

To study the possible modes of substrate binding within the dicopper core and to gain structural insight on the dicopper(II)-catecholate intermediate species formed during the catalytic reaction, tetrachlorocatechol (TCC) is usually employed as a structural mimic of the DTBC substrate, since TCC shows no tendency to undergo oxidation. Various mononuclear copper-catecholate complexes were structurally characterized, ${ }^{171}$ while only few examples of dicopper systems with coordinated TCC have been reported. ${ }^{170,71}$

Several different binding modes for TCC within the bimetallic pocket are possible (Scheme 38): the $\eta^{1}$-mode represents monodentate coordination to only one of the copper ions (a), the $\eta^{2}$-mode, in which TCC acts as a bidentate ligand (b) which may be complemented by Hbonding to a solvent molecule of the second copper ion (c), and $\mu-\eta^{1}: \eta^{1}$-mode, which represents a bridging coordination to both copper ions of a dinuclear complex (d).

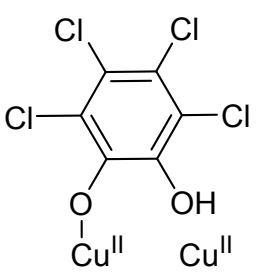

$\eta^{1}$

a

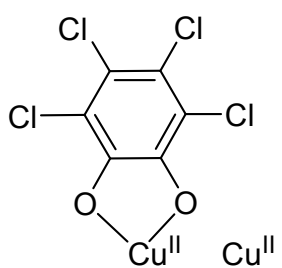

$\eta^{2}$

b<smiles>[R]Oc1c(Cl)c(Cl)c(Cl)c(Cl)c1O[Al]</smiles>

C<smiles>ClCOc1c(Cl)c(Cl)c(Cl)c(Cl)c1OCl</smiles>

$\mu-\eta^{1}: \eta^{1}$

d

Scheme 38. Possible binding motifs of TCC within a dicopper core.

In order to investigate the coordination behaviour of the dicopper complex 5a towards not only phenols but also $o$-diphenols, and to extend the library of the possible modes of substrate binding for dicopper complexes with pyrazolate-based ligands, TCC was reacted with the newly synthesized bioinspired dicopper(II) complex 5a. To a solution of $\mathbf{H L}^{4}$ in methanol 
were added two equivalents of $\mathrm{KO} t \mathrm{Bu}$ and subsequently, after $10 \mathrm{~min}$ of stirring, two equivalents of $\mathrm{Cu}\left(\mathrm{ClO}_{4}\right)_{2} \cdot 6 \mathrm{H}_{2} \mathrm{O}$. The reaction was completed by addition of 1 equivalent of tetrachlorocatechol, which was pretreated with 1 equivalent of $\mathrm{KO} t \mathrm{Bu}$ to deprotonate one of the $\mathrm{OH}$-groups. The colour of the reaction mixture changed from green to dark-brown. After the solvent was evaporated, the crude residue was dissolved in a MeCN/DMF mixture from which single crystals of complex 14 were obtained upon slow diffusion of $\mathrm{Et}_{2} \mathrm{O}$. The molecular structure of the cationic part of $\mathbf{1 4}$ is shown in Figure 82.

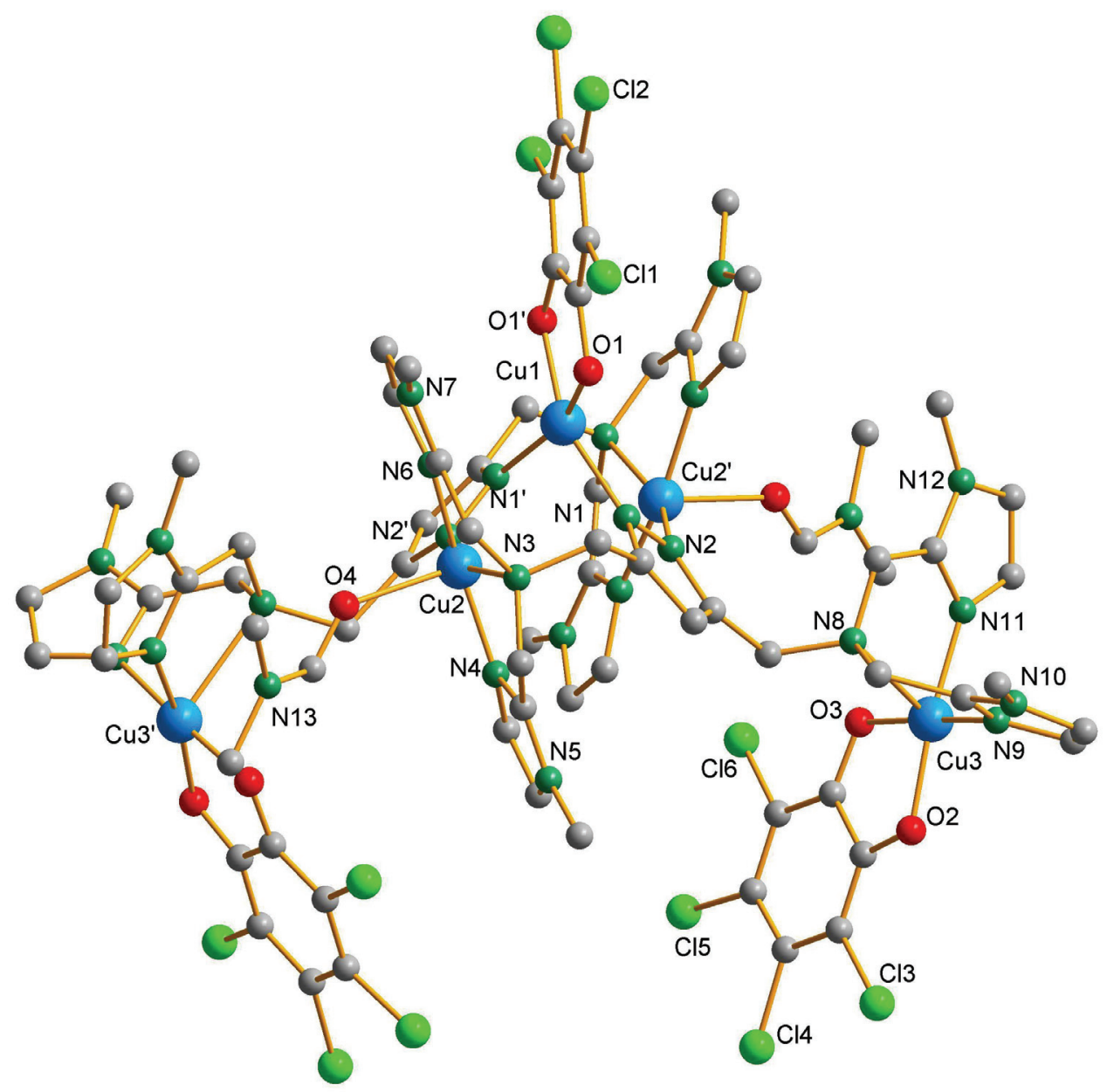

Figure 82. Molecular structure of the cation of $\mathbf{1 4}$. 


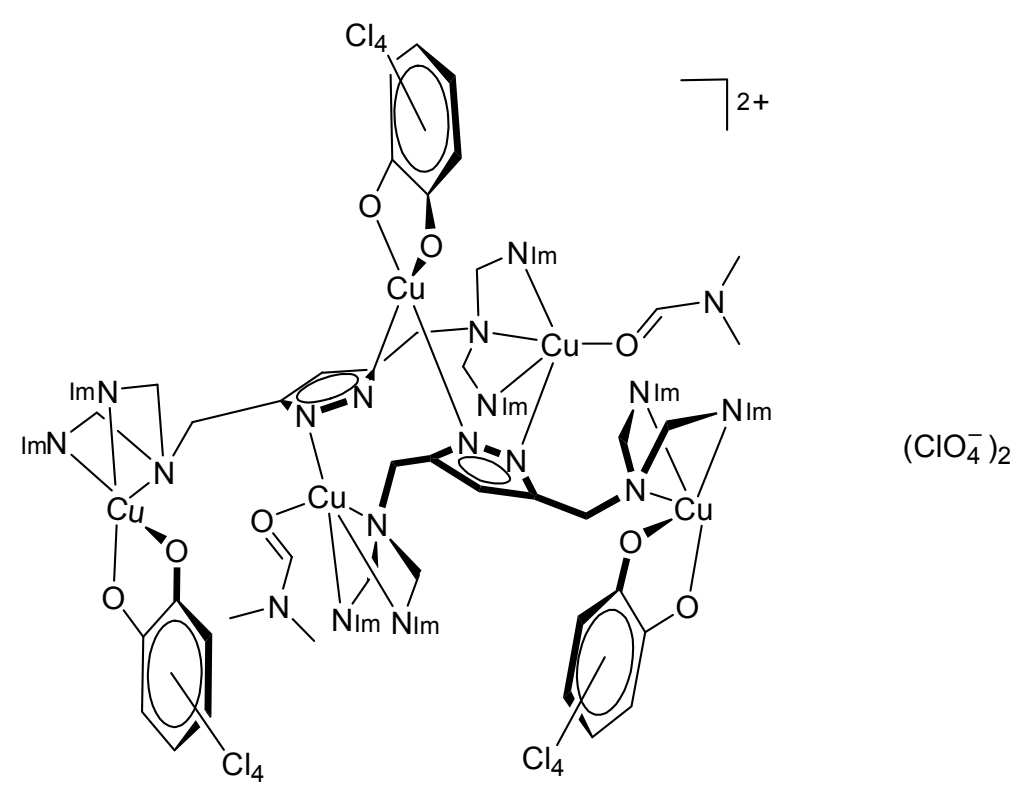

Figure 83. Schematic representation of complex 14: for clarity the $N$-methylimidazole is depicted as $\mathrm{N}_{\mathrm{Im}}$.

Table 20. Selected intramolecular distances $(\AA)$ and angles $\left(^{\circ}\right)$ for complex 14.

\begin{tabular}{llll}
\hline \multicolumn{4}{c}{ Distances } \\
\hline $\mathrm{Cu}(1)-\mathrm{O}(1)^{\prime}$ & $1.912(4)$ & $\mathrm{Cu}(3)-\mathrm{O}(3)$ & $1.931(4)$ \\
$\mathrm{Cu}(1)-\mathrm{O}(1)$ & $1.912(4)$ & $\mathrm{Cu}(3)-\mathrm{N}(11)$ & $1.979(5)$ \\
$\mathrm{Cu}(1)-\mathrm{N}(1)$ & $1.987(4)$ & $\mathrm{Cu}(3)-\mathrm{N}(9)$ & $1.982(5)$ \\
$\mathrm{Cu}(1)-\mathrm{N}(1)^{\prime}$ & $1.987(4)$ & $\mathrm{Cu}(3)-\mathrm{N}(8)$ & $2.470(4)$ \\
$\mathrm{Cu}(2)-\mathrm{N}(4)$ & $1.942(4)$ & $\mathrm{Cu}(1) \cdots \mathrm{Cu}(2)$ & $3.1801(8)$ \\
$\mathrm{Cu}(2)-\mathrm{N}(6)$ & $1.951(4)$ & $\mathrm{Cu}(1) \cdots \mathrm{Cu}(3)$ & $8.3560(9)$ \\
$\mathrm{Cu}(2)-\mathrm{N}(2)^{\prime}$ & $1.965(4)$ & $\mathrm{Cu}(2)^{\cdots} \cdot \mathrm{Cu}(3)$ & $9.0634(9)$ \\
$\mathrm{Cu}(2)-\mathrm{N}(3)$ & $2.106(4)$ & $\mathrm{Cu}(2)^{\cdots} \cdot \mathrm{Cu}(2)^{\prime}$ & $4.955(1)$ \\
$\mathrm{Cu}(2)-\mathrm{O}(4)$ & $2.312(3)$ & $\mathrm{Cu}(3)^{\cdots} \cdot \mathrm{Cu}(3)^{\prime}$ & $12.817(1)$ \\
$\mathrm{Cu}(3)-\mathrm{O}(2)$ & $1.920(4)$ & $\mathrm{Cu}(2)^{\cdots} \mathrm{Cu}(3)^{\prime}$ & $5.9133(9)$ \\
\hline & & & \\
\hline $\mathrm{O}(1)^{\prime}-\mathrm{Cu}(1)-\mathrm{O}(1)$ & $86.8(2)$ & $\mathrm{N}(6)-\mathrm{Cu}(2)-\mathrm{O}(4)$ & $82.71(14)$ \\
$\mathrm{O}(1)^{\prime}-\mathrm{Cu}(1)-\mathrm{N}(1)$ & $156.90(15)$ & $\mathrm{N}(2)^{\prime}-\mathrm{Cu}(2)-\mathrm{O}(4)$ & $115.21(15)$ \\
$\mathrm{O}(1)-\mathrm{Cu}(1)-\mathrm{N}(1)$ & $93.62(17)$ & $\mathrm{N}(3)-\mathrm{Cu}(2)-\mathrm{O}(4)$ & $88.44(14)$ \\
$\mathrm{O}(1)^{\prime}-\mathrm{Cu}(1)-\mathrm{N}(1)^{\prime}$ & $93.62(17)$ & $\mathrm{O}(2)-\mathrm{Cu}(3)-\mathrm{O}(3)$ & $85.50(17)$ \\
$\mathrm{O}(1)-\mathrm{Cu}(1)-\mathrm{N}(1)^{\prime}$ & $156.90(15)$ & $\mathrm{O}(2)-\mathrm{Cu}(3)-\mathrm{N}(11)$ & $169.61(18)$
\end{tabular}




\begin{tabular}{llll}
$\mathrm{N}(1)-\mathrm{Cu}(1)-\mathrm{N}(1)$ & $94.8(2)$ & $\mathrm{O}(3)-\mathrm{Cu}(3)-\mathrm{N}(11)$ & $91.34(18)$ \\
$\mathrm{N}(4)-\mathrm{Cu}(2)-\mathrm{N}(6)$ & $163.30(19)$ & $\mathrm{O}(2)-\mathrm{Cu}(3)-\mathrm{N}(9)$ & $91.76(19)$ \\
$\mathrm{N}(4)-\mathrm{Cu}(2)-\mathrm{N}(2)$ & $98.94(17)$ & $\mathrm{O}(3)-\mathrm{Cu}(3)-\mathrm{N}(9)$ & $175.04(17)$ \\
$\mathrm{N}(6)-\mathrm{Cu}(2)-\mathrm{N}(2)$ & $97.51(17)$ & $\mathrm{N}(11)-\mathrm{Cu}(3)-\mathrm{N}(9)$ & $92.1(2)$ \\
$\mathrm{N}(4)-\mathrm{Cu}(2)-\mathrm{N}(3)$ & $82.45(17)$ & $\mathrm{O}(2)-\mathrm{Cu}(3)-\mathrm{N}(8)$ & $112.82(16)$ \\
$\mathrm{N}(6)-\mathrm{Cu}(2)-\mathrm{N}(3)$ & $81.35(17)$ & $\mathrm{O}(3)-\mathrm{Cu}(3)-\mathrm{N}(8)$ & $100.43(15)$ \\
$\mathrm{N}(2){ }^{\prime}-\mathrm{Cu}(2)-\mathrm{N}(3)$ & $156.09(15)$ & $\mathrm{N}(11)-\mathrm{Cu}(3)-\mathrm{N}(8)$ & $77.48(16)$ \\
$\mathrm{N}(4)-\mathrm{Cu}(2)-\mathrm{O}(4)$ & $92.83(14)$ & $\mathrm{N}(9)-\mathrm{Cu}(3)-\mathrm{N}(8)$ & $76.82(17)$ \\
\hline
\end{tabular}

Surprisingly, an unusual pentanuclear copper complex was isolated instead of the expected dinuclear species. This can be explained by the significant flexibility of the pyrazolate ligand system, especially in the case of a polydentate imidazolyl side arms. These chelate arms incorporated into ligand $\mathbf{H L}^{4}$ possess a very high affinity towards copper, which in some cases results in coordination of copper ions solely in the side arm unit without involving the pyrazole core, as observed in $\mathbf{6}$, for example. Such a coordination motif induces a dimerization of the dinuclear species formed or even leads to more complicated structural features as observed in $\mathbf{1 4 .}$

Two ligands and five copper atoms are incorporated in the molecular structure of complex 14 and moreover, three TCC molecules are bound to three of the copper atoms. The structure possesses overall $C_{2}$-symmetry. One of the copper atoms $(\mathrm{Cu} 1)$ has a distorted tetragonal geometry, whilst being coordinated to N-atoms of two different pyrazolate units. The coordination sphere is completed by a TCC molecule which is $\eta^{2}$-coordinated in the bidentate binding mode. A copper atom of the second type $(\mathrm{Cu} 2)$ is ligated by four $\mathrm{N}$-atoms (three from the side arm of one ligand subunit and an additional pyrazole $\mathrm{N}$-atom of the second ligand involved) and one additional oxygen atom from a DMF molecule used for crystallization, resulting in almost perfect square pyramidal geometry $(\tau=0.11)$. The remaining side arms of the two ligands have rotated away from the pyrazole core and host the remaining copper atom (Cu3), whose coordination sphere consists of three side arm $\mathrm{N}$ atoms. An overall square pyramidal geometry $(\tau=0.11)$ of this outer metal ion is completed by the $\eta^{2}-$ bound TCC molecule.

Signals at 1183, 937, 872 and $627 \mathrm{~m} / \mathrm{z}$ were observed in the ESI MS spectrum of a MeCN solution of 14, which confirm the presence of the species $\left[\mathrm{Cu}_{3} \mathrm{~L}^{4}(\mathrm{TCC})_{2}\right]^{+}$, $\left[\mathrm{Cu}_{2} \mathrm{~L}^{4}(\mathrm{TCC})(\mathrm{MeOH})_{2}\right]^{+},\left[\mathrm{Cu}_{2} \mathrm{~L}^{4}(\mathrm{TCC})\right]^{+}$and $\left[\mathrm{Cu}_{2} \mathrm{~L}^{4}\right]^{+}$, respectively. The structural features were perfectly reflected in the UV/vis spectrum in MeCN solution, where two $d$ - $d$ transition 
bands were observed: a typical $d-d$ band for a tetragonally coordinated copper center (Cu1) with $\lambda_{\max }=917 \mathrm{~nm}$, and a high energy $d-d$ transition for square pyramidal copper ions $(\mathrm{Cu} 2$ and $\mathrm{Cu} 3$ ) with a band at $\lambda_{\max }=702 \mathrm{~nm}$.

The magnetic susceptibility of a powdered sample of $\mathbf{1 4}$ was measured at two different magnetic fields $(2000 \mathrm{G}$ and $5000 \mathrm{G})$ in a temperature range from $2.0 \mathrm{~K}$ to $295 \mathrm{~K}$. No significant field dependence was observed. The temperature dependence of the effective magnetic moment $\mu_{\text {eff }}$ is depicted in Figure 84.



Figure 84. Plot of $\mu_{\text {eff }}$ versus temperature at $2000 \mathrm{G}$ for 14.

The magnetic moment of $3.86 \mu_{\mathrm{B}}$ at $295 \mathrm{~K}$ closely matches the value expected for five uncoupled copper(II) ions (Scheme 39) while upon decreasing the temperature to $2 \mathrm{~K}$, only three uncoupled copper(II) ions remained $\left(3.00 \mu_{\mathrm{B}}\right)$.



Scheme 39. A qualitative picture showing the alignment of the spin moment of the electrons in the pentanuclear copper complex 14. 
The observed antiferromagnetic interaction can take place only between three copper atoms bridged by a pyrazole unit $((d(\mathrm{Cu} 1 \cdots \mathrm{Cu} 2)=3.18 \AA)$ resulting in a spin value of $1 / 2$. Two outer copper atoms $(\mathrm{Cu} 3)$ remained uncoupled and the overall spin value for the whole system at low temeperature is $3 / 2$. 


\section{Oxidative ortho-C-C coupling of 4-ethylphenol catalyzed by 8}

In marked contrast to the observed selective oxidative $\mathrm{C}-\mathrm{C}$ coupling of TMP in the paraposition to yield TMSQ, the substrate 4-ethylphenol (from here on abbreviated as 4-EtP) was found to undergo $\mathrm{C}-\mathrm{C}$ coupling in the ortho-position yielding an unique oligomeric compound o,o-4-EtP (Scheme 40). Apparently, steric congestion at the para substituent in 4-EtP favors C-C coupling in the ortho-position to occur. Therefore, the only accessible positions for any C-C coupling are the carbons that are in the ortho-positions to the phenol, as coupling in meta-position is electronically not preferable in terms of stability of a reactive intermediate (cf. resonance structures of aromatic substitution reactions).

$\mathrm{n}$<smiles>CCc1ccc(O)cc1</smiles>

4-EtP



$\mathrm{MeCN} / \mathrm{MeOH}(1: 1)$

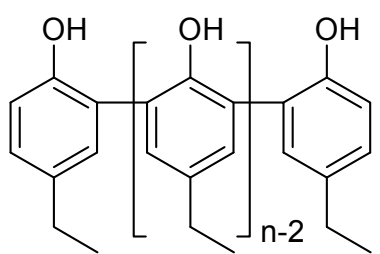

$0,0-4-E t P$

$n=2,3$

Scheme 40. Oxidative C-C coupling of 4-EtP in the ortho-position catalyzed by 8 .

The catalyst used for performing the oxidative C-C coupling of 4-EtP was based on the ligand $\mathbf{H L}^{\mathbf{5}}$ with a phenyl substituent in the backbone of the pyrazole core. Unfortunately, crystallization of the dinuclear copper(II) complexes derived from this ligand did not result in formation of crystals suitable for X-ray analysis (opposite to what was expected and intended when this ligand backbone was designed), and only in one case the tetranuclear complex $\mathbf{8}$ was structurally characterized. As discussed in section 5.5, this particular tetranuclear species is most likely present only in the solid state, and on the basis of ESI spectrometry a dissociation process is assumed to be operative in solution, resulting in the formation of dinuclear subunit $8^{\prime}$ with a $\mathrm{MeO} \cdots \mathrm{HOMe}$ or $\mathrm{HO}{ }^{\cdots} \mathrm{HOH}$ bridging motif (depending on the solvent used) within the bimetallic pocket (see Scheme 28 in section 5.5), as observed in 5a. This dinuclear species $\mathbf{8}^{\prime}$ ' was considered as the active species during the oxidative C-C coupling of 4-EtP. Contrary to the C-C coupling of TMP, for the observed coupling of 4-EtP to occur, the presence of one additional equivalent of external base $(\mathrm{KO} t \mathrm{Bu})$ is required. 
Addition of the substrate to a $\mathrm{MeCN}$ solution of $\mathbf{8}$ without additional base resulted in appearance of the copper(II)-phenolate CT band at $453 \mathrm{~nm}$. No significant shift of the copper $d-d$ transition band was observed, which is a typical feature for simple coordination of the phenol molecule within the bimetallic pocket via replacement of bound $\mathrm{H}_{2} \mathrm{O} / \mathrm{MeOH}$, and without any further oxidation occurring. Observation of the reaction mixture over time showed that the intensity of this CT band remained unchanged, indicating that this copper(II)phenolate species is unreactive. Unfortunately, studies on the stoichiometry between copper complex and 4-EtP by Job's method were unsuccessful due to significant dissociation of the phenolate species in solution under these conditions. ${ }^{1} \mathrm{H}$ NMR analysis of the reaction mixture after $24 \mathrm{~h}$ and after separation of all copper species passing the solution over a silica column revealed the presence of the starting 4-EtP as the only organic material. UV/vis studies were carried out with the reaction mixture including one additional equivalent of base. Addition of base led to a shift of the LMCT band to higher wavelength, with a maximum at $473 \mathrm{~nm}$, concurrent with an increase in the intensity of this band. These observations indicate a shift of the reaction equilibrium towards the formation of the copper(II)-phenolate species and therefore Job's method was applied to study the stoichiometry between $\mathbf{8}$ and the phenolate derived from 4-EtP. Unfortunately, this particular technique does not allow investigations on the interaction between the dicopper complex and 4-EtP in the presence of only one equivalent of base (as used for the reaction). Therefore, a Job experiment was performed using a solution of completely deprotonated 4-EtP. From the Job plot depicted in Figure 85 it was deduced that a maximum of two phenolate molecules can be coordinated to the dicopper core. 


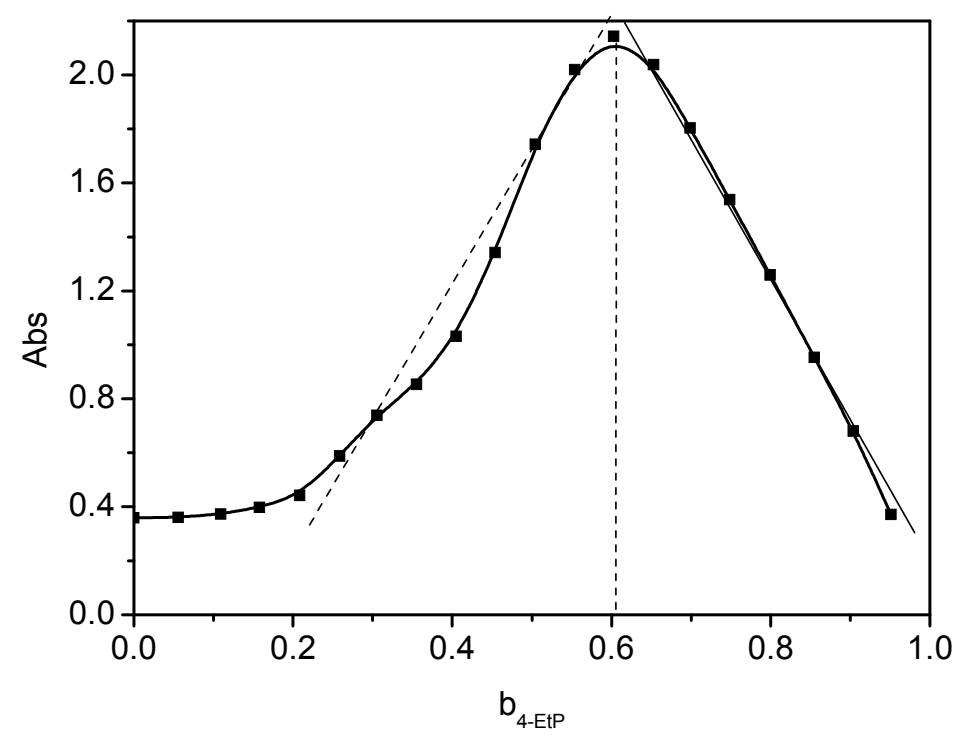

Figure 85. Job plot of 8 with $4-\mathrm{EtP}$ in $\mathrm{MeCN}, \lambda_{\max }=473 \mathrm{~nm}$.

To further study the influence of base on the reaction between 8 and 4-EtP, Raman spectroscopy was applied. First of all, the Raman spectrum of the reaction mixture without supplementary base was recorded (Figure 86 (a)).

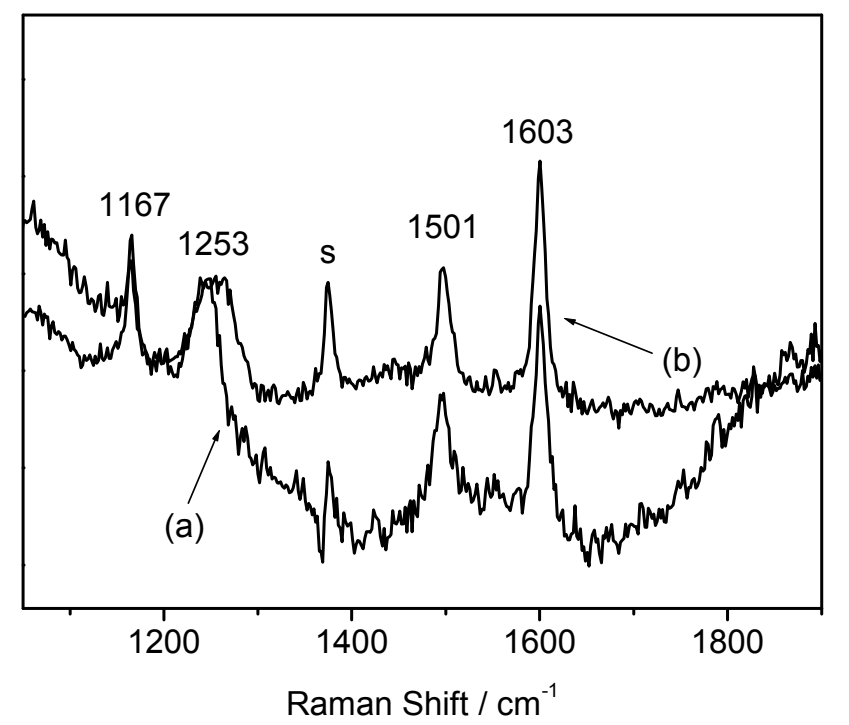

Figure 86. Resonance Raman spectra of the reaction mixture of 8 and 4-EtP in $\mathrm{MeCN} ; \lambda_{\mathrm{ex}}=$ $488 \mathrm{~nm}$; (a) mixture without $\mathrm{KO} t \mathrm{Bu}$; (b) mixture with one equivalent of $\mathrm{KO} t \mathrm{Bu}$; s denotes not fully subtracted solvent peak. 
Laser excitation was performed at $488 \mathrm{~nm}$, i.e. into the LMCT band of the copper(II)phenolate adduct at $453 \mathrm{~nm}$. Typical features were observed in the Raman spectrum for a phenolate coordinated to copper(II) (as for example observed in case of phenolate adduct with $\mathrm{M} t \mathrm{BuP}$, see section 7.4.1), with bands at 1167, 1253, 1501, and $1603 \mathrm{~cm}^{-1}$. Addition of base did not induce any changes in the Raman spectrum, proving that the identical dicopper(II)phenolate species is formed under both conditions (Figure 86 (b)).

Slow diffusion of $\mathrm{Et}_{2} \mathrm{O}$ into a solution of $\mathbf{8}$ and 4-EtP with one equivalent of $\mathrm{KO} t \mathrm{Bu}$ led to formation of the black crystals. The X-ray crystallographic measurements allowed only a rough estimation of the molecular structure, because of the presence of disordered solvent molecules for which no satisfactory model for the disorder could be found. The corresponding approximation of the molecular structure of $\mathbf{1 5}$ is shown in Figure 87.

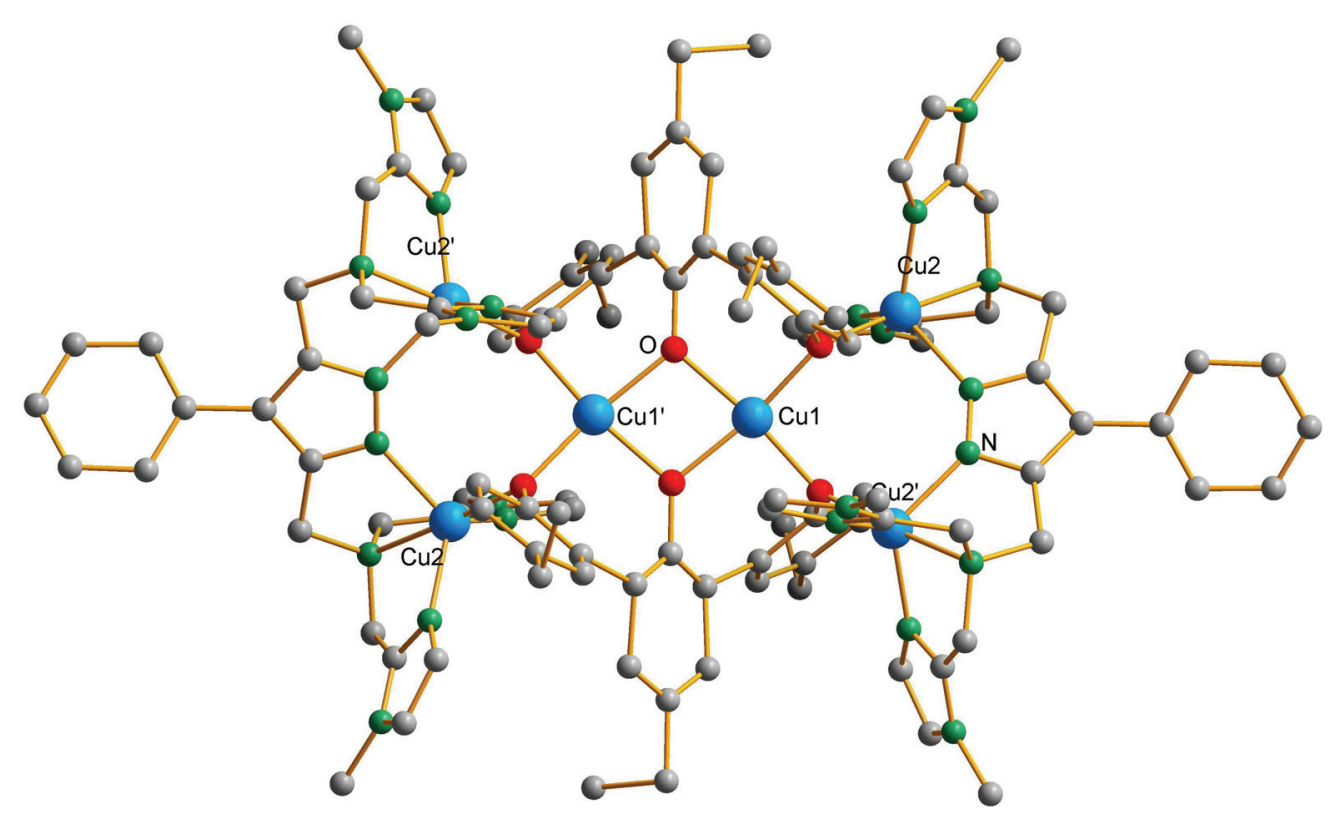

Figure 87. Molecular structure of the cationic part of complex 15. Average positions of the carbon atoms of the central phenolate unit were calculated from the disordered atomic positions, initially available from the X-ray data analysis.

The formed oligonuclear complex 15 consists of two outer pyrazolate-based dicopper subunits with the copper atoms ( $\mathrm{Cu} 2$ and $\mathrm{Cu} 2$ ') hosted within the chelate arm compartments and bridged by pyrazole, as anticipated. The coordination of $\mathrm{Cu} 2 / \mathrm{Cu} 2$ ' is completed by a phenolate-O atom from the central $\mathrm{Cu}_{2} \mathrm{O}_{6}$ part of the complex, which has apparently been formed via C-C coupling of 4-EtP. Coupling in both ortho-positions of the central 4-EtP 
fragment yielded a unique 2,2 ', 5',2' '-triphenol species that acts as a bridging ligand for all six copper atoms present in the molecular structure. Each phenolate unit bridges two copper ions, resulting in a distorted square planar geometry for the two copper atoms located in the center of the molecule (Figure 88).

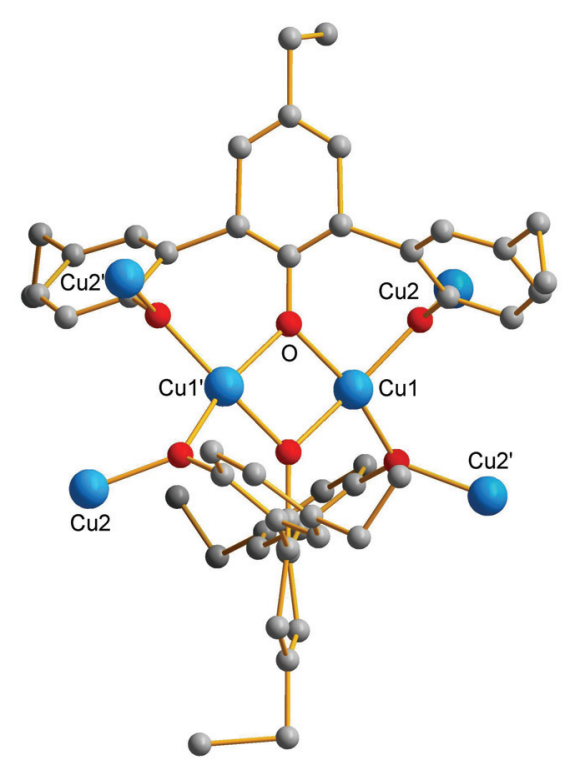

Figure 88. Selected part of the cation of the molecular structure of complex 15: Nitrogen- and carbon-atoms of the pyrazole ligands are omitted for clarity.

Stability of such a hexanuclear species in solution was proven by means of ESI high resolution mass spectrometry, whereby a doubly charged cation $\left[\mathrm{M}-2\left(\mathrm{ClO}_{4}\right)^{-}\right]^{2+}(\mathrm{M}$ is complex 15) was detected at $1224.23 \mathrm{~m} / \mathrm{z}$ (calcd. $1224.22790 \mathrm{~m} / \mathrm{z}$ (Figure 89)).
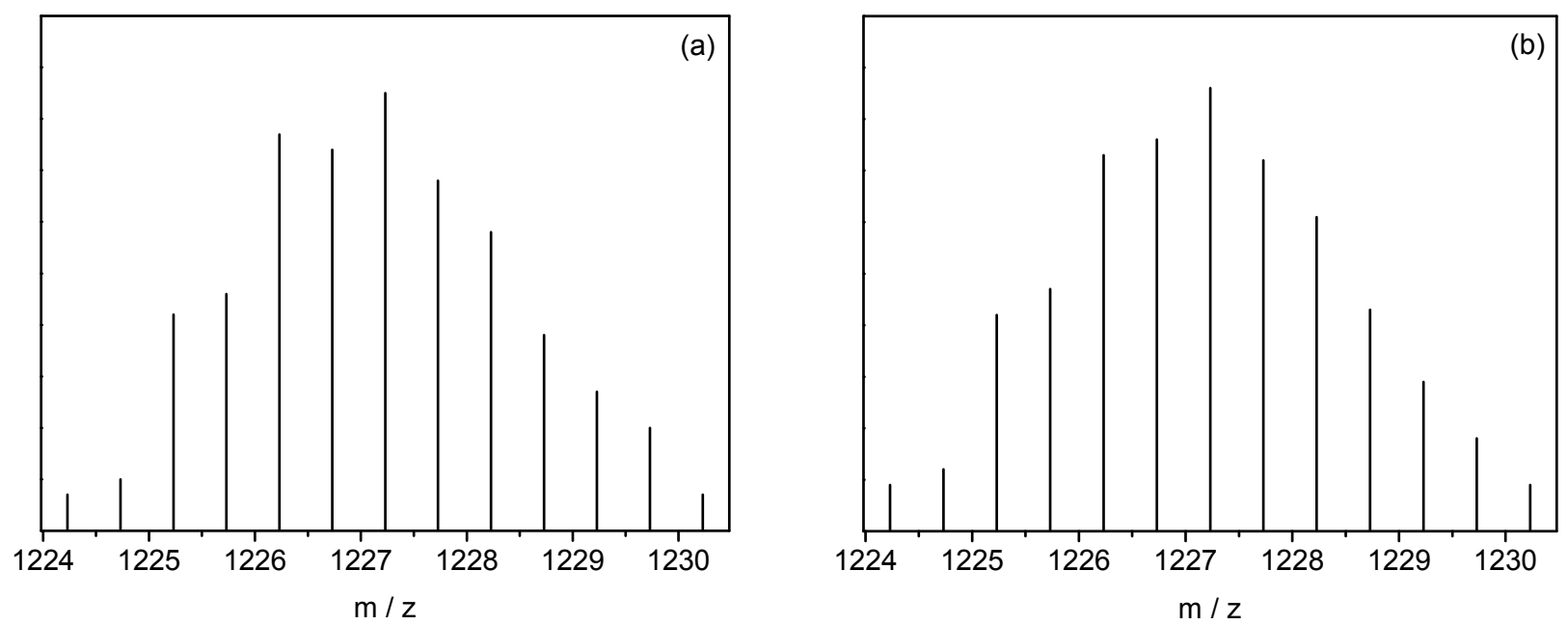

Figure 89. High resolution ESI-spectrum of complex 15 in MeCN: (a) observed signal; (b) simulated isotope pattern for the fragment $\left[\mathrm{M}-2\left(\mathrm{ClO}_{4}\right)^{-}\right]^{2+}$. 
The UV/vis spectrum in the solid state (diffuse reflectance) showed three signals at $\lambda_{\max }$ of 427, 633 and $1039 \mathrm{~nm}$, which are in good agreement with the coordination environmets of the copper ions found in the crystal structure. Thus, the signal at $1039 \mathrm{~nm}$ corresponds to the $d-d$ transition band of the copper ions in a trigonal bipyramidal geometry $(\mathrm{Cu} 2, \mathrm{Cu} 2$ '), while the absorption maximum at $633 \mathrm{~nm}$ is typically assigned to a $d$-d band for copper in a square planar coordination mode ( $\left.\mathrm{Cu} 1, \mathrm{Cu} 1^{\prime}\right)$. The coordinated triphenol unit implies the presence of a copper-to-phenolate CT transition band, found in the solid state UV/vis spectrum at $427 \mathrm{~nm}$. These spectroscopic features were also found in some other copper complexes with incorporated phenolate subunits. ${ }^{172}$ The UV/vis spectrum of an acetonitrile solution of $\mathbf{1 5}$ showed only two $d$ - $d$ bands at 972 and $732 \mathrm{~nm}$ for the copper ions in a trigonal bipyramidal and square planar coordination mode, respectively. The observed differences in UV/vis spectra in the solid state and solution imply some structural preorganization in the hexanuclear copper complex 15 upon dissolving.

Analysis of the reaction mixture after crystallization of this complex by means of GC-MS spectrometry revealed the presence of 4-EtP $(122 \mathrm{~m} / \mathrm{z})$ and three isomers of the dimeric derivative of 4-EtP at $242 \mathrm{~m} / \mathrm{z}$. The small difference observed in the fragmentation of these isomers (for two of these isomers loss of a $\mathrm{CH}_{3}$-group was observed, while in the third a $\mathrm{C}_{2} \mathrm{H}_{5}$-group was split off) did not allow the determination of the exact structure of the three isomers. Based on the studies with $p$-cresol reported in literature ${ }^{173}$ it can be assumed that during the oxidative ortho-C-C coupling of 4-EtP different coupling modes have taken place resulting in the formation or three isomers depicted in Figure 90.

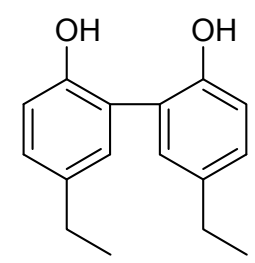

(a)

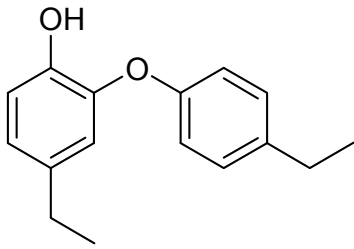

(b)

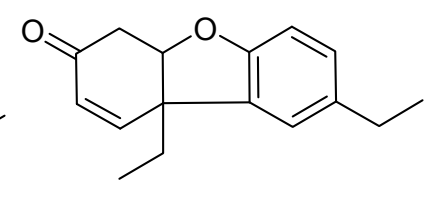

(c)

Figure 90. Proposed structures of three isomers of the dimeric derivative of 4-EtP.

There are three most probable possibilities to form dimers during the C-C coupling, i.e. an ortho-ortho $\mathrm{C}-\mathrm{C}$ coupling to form dimer (a), an ortho $\mathrm{C}-\mathrm{O}-\mathrm{C}$ coupling to yield dimmer (b), and an ortho-para C-C coupling to form a structural analogue to the Pummerer's ketone. The trimeric product derived from 4-ethylphenol $(362 \mathrm{~m} / \mathrm{z})$, which was found in the crystal structure, was detected only in traces, while any other oligomeric compounds derived from 4- 
EtP were not observed in the GC-MS spectrum. These oligomeric compounds may did not form at all or their absence can either be caused by (unexpected) strong interaction of these oligomers with the column material used for the measurements or a low tendency of these species to become ionized, resulting in insufficiently low concentrations for detection in the GC-MS set-up.

On the basis of these results, it can be concluded that C-C coupling of the substrate 4-EtP preferably occurs in the ortho-position, as was found in the crystal structure of 15. Most likely, this reaction mechanistically proceeds via an initially formed bisphenol species, as detected by GC mass spectrometry. 


\section{Nucleophilic 1,6-addition of MeOH to 2,4,6-trimethylphenol}

Derivatives of 2,4,6-trimethylphenol functionalized at the 4-position are very important organic compounds for industrial purposes ${ }^{174,175}$ as well as for fundamental research. ${ }^{176,177}$ It is known that the synthetic pathway to produce para-substituted 2,4,6-trimethylphenol derivatives may involve a benzoquinone methide intermediate (Scheme 41 (a)). ${ }^{99}$

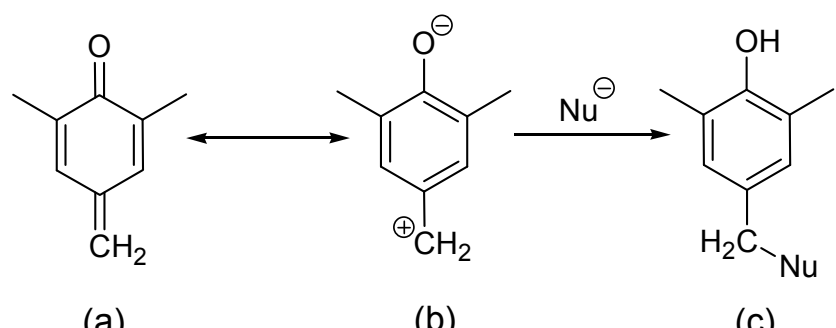

(a)

(b)

(c)

Scheme 41. Benzoquinone methide (a), its zwitterionic resonance form (b), para-substituted product after nucleophilic addition (c).

para-Benzoquinone methide exists only as a short-lived species in dilute solution, and has a limited stability. ${ }^{178}$ Considering the existence of such a highly reactive para-benzoquinone methide species, formation of 4-methoxy-2,6-dimethylphenol could be explained as a result of nucleophilic attack by methoxide, formed from a deprotonated methanol molecule, to a quinone methide. ${ }^{99}$ Work previously reported by Reedijk and co-workers on the nucleophilic addition to TMP made use of the copper system $\left.\left[\mathrm{CuCl}_{2} \text {-(neocuproine) }\right)_{2}-\mathrm{NaOMe}\right]$ to mediate a four-electron process to convert TMP, via ketal formation and subsequent hydrolysis, to yield 4-hydroxy-3,5-dimethylbenzaldehyde XVII (see section 3.3, Scheme 15).

2,4,6-Trimethylphenol, which undergoes selective oxidative C-C coupling catalyzed by dicopper(II) complex 5a in $\mathrm{MeCN} / \mathrm{CH}_{2} \mathrm{Cl}_{2}$ (see section 6.3), can yield distinctly different products upon changing the reaction conditions, most notably by varying the reaction medium. Thus, addition of $\mathrm{MeOH}$ to a reaction mixture of $\mathbf{5 a}$ and TMP in MeCN after 10 minutes of reaction time led to 1,6-nucleophilic addition of $\mathrm{MeOH}$ to the methyl-group of TMP in the para-position, resulting in the formation of 4-methoxy-2,6-dimethylphenol (MDP) (Scheme 42). 


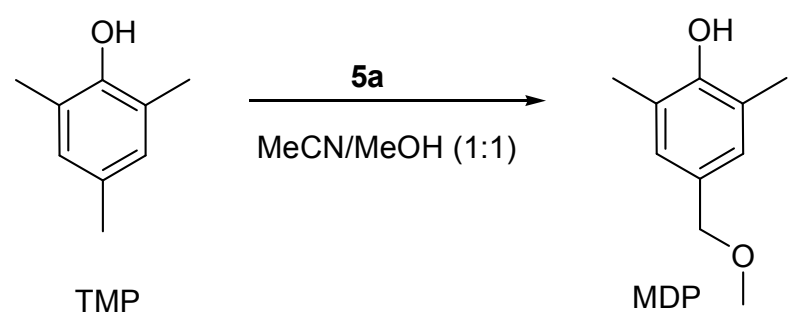

Scheme 42. 1,6-nucleophilic addition of MeOH to TMP catalyzed by $\mathbf{5 a}$.

Standard reaction conditions as for the oxidative C-C coupling of TMP were initially used to perform the 1,6-nucleophilic addition of $\mathrm{MeOH}$ to TMP. $4 \mathrm{ml}$ of $\mathrm{MeOH}$ was added after 10 minutes to a reaction mixture of $\mathbf{5 a}$ and five equivalents of TMP in $4 \mathrm{ml}$ of MeCN. The yield of 4-methoxy-2,6-dimethylphenol was determined by means of ${ }^{1} \mathrm{H}-\mathrm{NMR}$ spectroscopy, using 1,3,5-trimethoxybenzene as internal standard. Formation of MDP was established after detection of the characteristic signal in the ${ }^{1} \mathrm{H}$ NMR spectrum at $3.34 \mathrm{ppm}$ for the $\mathrm{CH}_{3}$-group of the resulting ether fragment. The reaction was followed for $60 \mathrm{~h}$ after which time MDP was formed in $48 \%$ yield, corresponding to a TON of 2.5 (Figure 91 (a)). Together with the formation of 4-methoxy-2,6-dimethylphenol as the major product, traces of 4-hydroxy-3,5dimethylbenzaldehyde were observed. Use of a bigger excess of TMP (50 eq.) led to formation of MDP with a total turnover number of 12, after $60 \mathrm{~h}$ (Figure 91 (b)).

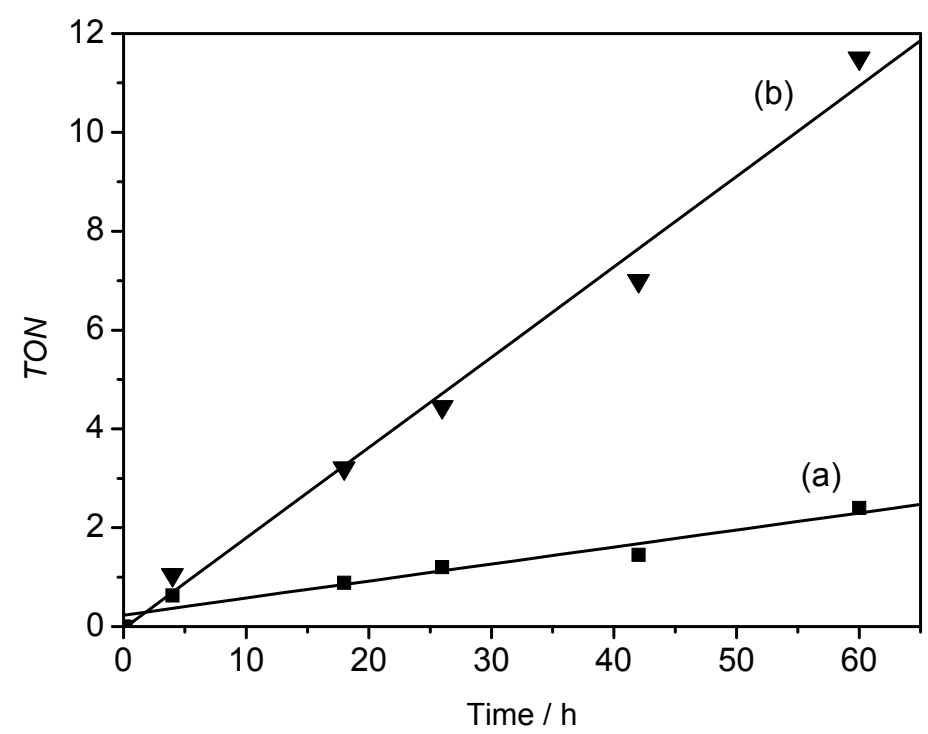

Figure 91. Turnover numbers for formation of MDP: (a) 5 equivalents TMP; (b) 50 equivalents TMP. 
As was previously described (see section 5.4.2), dicopper complex 5a contains a $\mathrm{MeO}$.* HOMe bridge within the bimetallic pocket. It was furthermore established that upon coordination of the phenolic substrate to one of the copper(II) ions, the OH-group of the phenol becomes deprotonated due to the presence of the methoxide ligand that is acting as a internal base, and a methanol molecule is released. In order to prove that 1,6-addition of 'exogenous' methanol added to the reaction mixture was taking place, $\mathrm{CD}_{3} \mathrm{OD}$ was used as nucleophile. The reaction was performed under the same conditions, using 5 equivalents excess of TMP but instead $\mathrm{CD}_{3} \mathrm{OD}$ was added after $10 \mathrm{~min}$ of reaction time (Scheme 43).

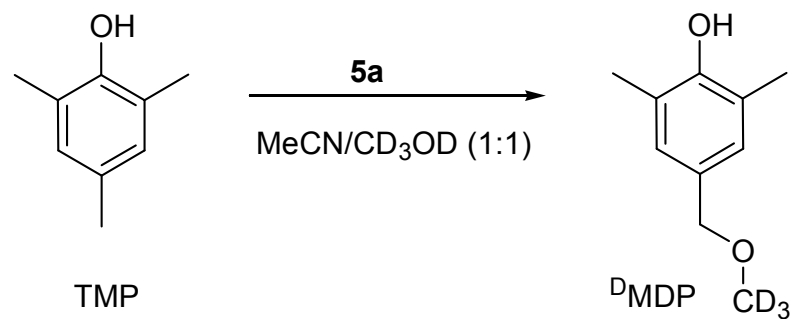

Scheme 43. 1,6-nucleophilic addition of $\mathrm{CD}_{3} \mathrm{OD}$ to TMP catalyzed by $\mathbf{5 a}$.

Analysis of the reaction mixture by ${ }^{1} \mathrm{H}$ NMR spectroscopy after $40 \mathrm{~h}$ confirmed the formation of ${ }^{D}$ MDP, where nucleophilic addition on the para-methyl group has occurred with incorporation of the fully deuterated $\mathrm{OCD}_{3}$-group, resulting in the complete absence of the signal for the protons of the $\mathrm{OCH}_{3}$-group at $3.34 \mathrm{ppm}$, while again only traces of 4-hydroxy3,5-dimethylbenzaldehyde were detected.

The observed 1,6-addition of $\mathrm{MeOH}$ and $d^{4}-\mathrm{MeOH}$ catalyzed by dicopper complex 5a shows the significant potential of this complex to act as a versatile catalyst in different kinds of phenol oxidations besides the C-C coupling reaction, which can be used in the future to generate new organic compounds, not only by means of $\mathrm{C}-\mathrm{C}$ bond formation but in particular also by $\mathrm{C}-\mathrm{N}$ and $\mathrm{C}-\mathrm{O}$ bond making reactions. 


\section{Oxidative C-O coupling of 4-bromo-2,6-dimethylphenol catalyzed by} $5 a$

4-bromo-2,6-dimethylphenol (from here on abbreviated as DMBrP), which was initially applied as a model substrate to study phenol binding to the dicopper complex $\mathbf{5 a}$, since it was thought to be "inert" and should not participate in any $\mathrm{Cu}$-mediated catalytic reaction, was in fact shown to undergo oxidative $\mathrm{C}-\mathrm{O}$ coupling to yield a oligomeric compound Br-PPE in the presence of $\mathbf{5 a}$ and dioxygen (Scheme 44).

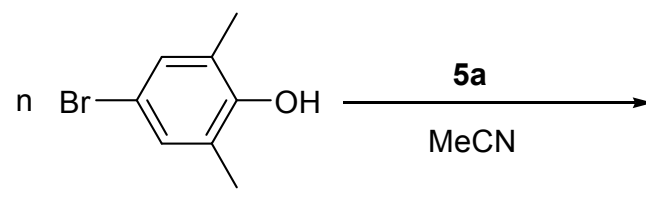

$\mathrm{DMBrP}$

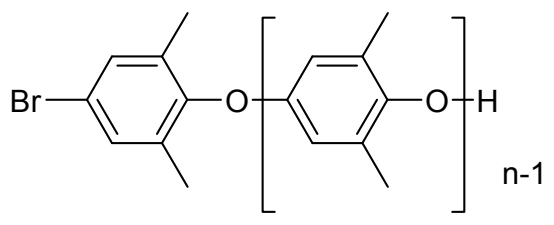

Br-PPE

Scheme 44. Oxidative C-O coupling of DMBrP catalyzed by $\mathbf{5 a}$.

The reaction was followed in time by means of UV/vis spectroscopy. The electronic spectrum of the green solution of 5a in MeCN showed a $d-d$ band at $\lambda_{\max } 989 \mathrm{~nm}$ with a shoulder at 724 $\mathrm{nm}$, corresponding to transitions of copper(II) ions in a trigonal bipyramidal environment (Figure 92 (a)). Addition of the DMBrP led to an immediate colour change from green to red, reflected in the UV/vis spectrum through the appearance of an intense charge-transfer band at $\lambda_{\max } 465 \mathrm{~nm}$ and a shift of the $\mathrm{Cu}^{\mathrm{II}}$-based $d$ - $d$ transition band to $960 \mathrm{~nm}$ (Figure 92(b)). These observations indicate that also with this substrate a dicopper(II)-phenolate species 16 (Scheme 45 ) is formed through deprotonation and exchange with the methanolate fragment. 




Figure 92. UV/vis spectra at different stages of the oxidative C-O coupling of DMBrP (initial concentration of $\mathbf{5 a}=0.001 \mathrm{~mol} \cdot \mathrm{L}^{-1}$; initial concentration of $\mathrm{DMBrP}=0.021 \mathrm{~mol} \cdot \mathrm{L}^{-1} ; \mathrm{L}=1.0$ cm; solvent: MeCN: (a) starting solution of 5a; (b) a few seconds after addition of DMBrP (red solution); (c) 12 min after addition of DMBrP.
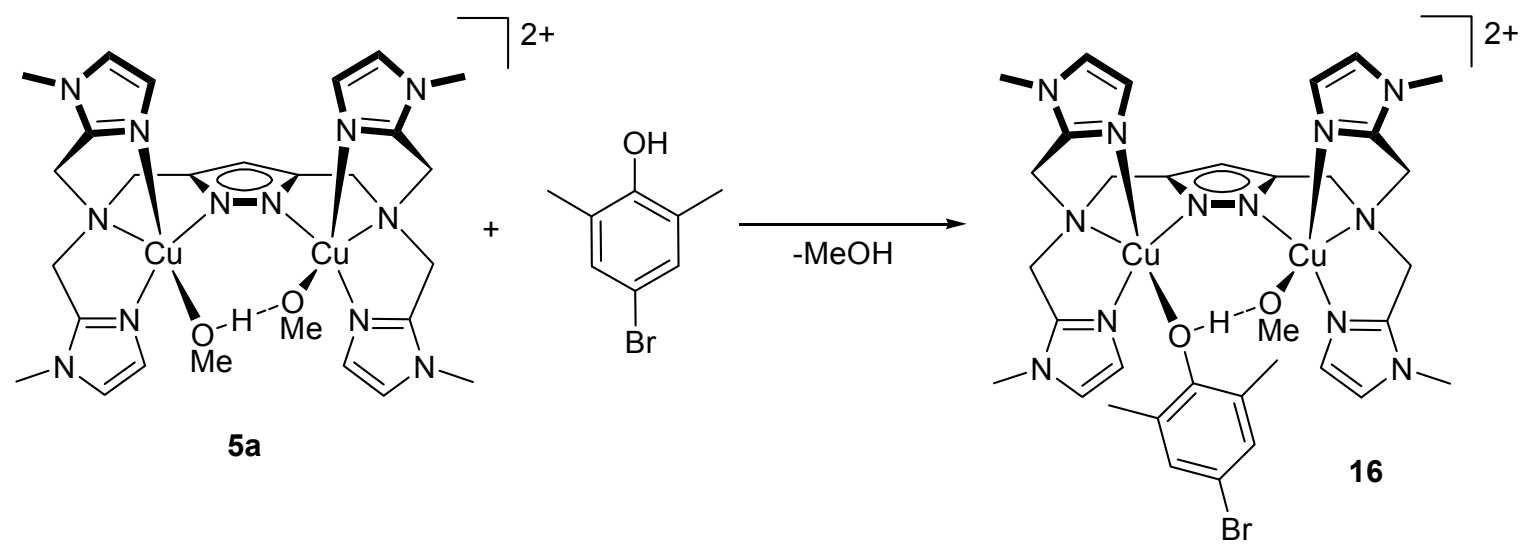

Scheme 45. Coordination of the DMBrP within the bimetallic pocket of $\mathbf{5 a}$.

Somewhat surprisingly and in contrast to the results obtained with TMP as the substrate (see section 6.3), rapid disappearance of the formed copper(II)-phenolate species occurred within 10 minutes, concomitant with a colour change of the reaction mixture to deep green (Figure 92 (c)). The UV/vis spectrum of the resultant solution showed the typical $d$ - $d$ transition band at $\lambda_{\max } 695 \mathrm{~nm}$ for a copper(II) ion in square pyramidal coordination environment. Polymeric $\mathrm{Br}-\mathrm{PPE}$ precipitated from the green solution as a white powder within the next 10 minutes. 
Analysis of the white precipitate by means of EI mass spectrometry proved the polymeric nature of the material, as it was found that the polymer chain consisted of at least ten phenol ether moieties with a peak at $1282 \mathrm{~m} / \mathrm{z}$, which corresponds to $\left[\mathrm{C}_{80} \mathrm{H}_{80} \mathrm{O}_{10} \mathrm{Br}\right]^{+}$. Further studies on the exact polymer composition were not performed.

Slow evaporation of the deep green solution at room temperature led to the accumulation of blue crystals, suitable for X-ray analysis.

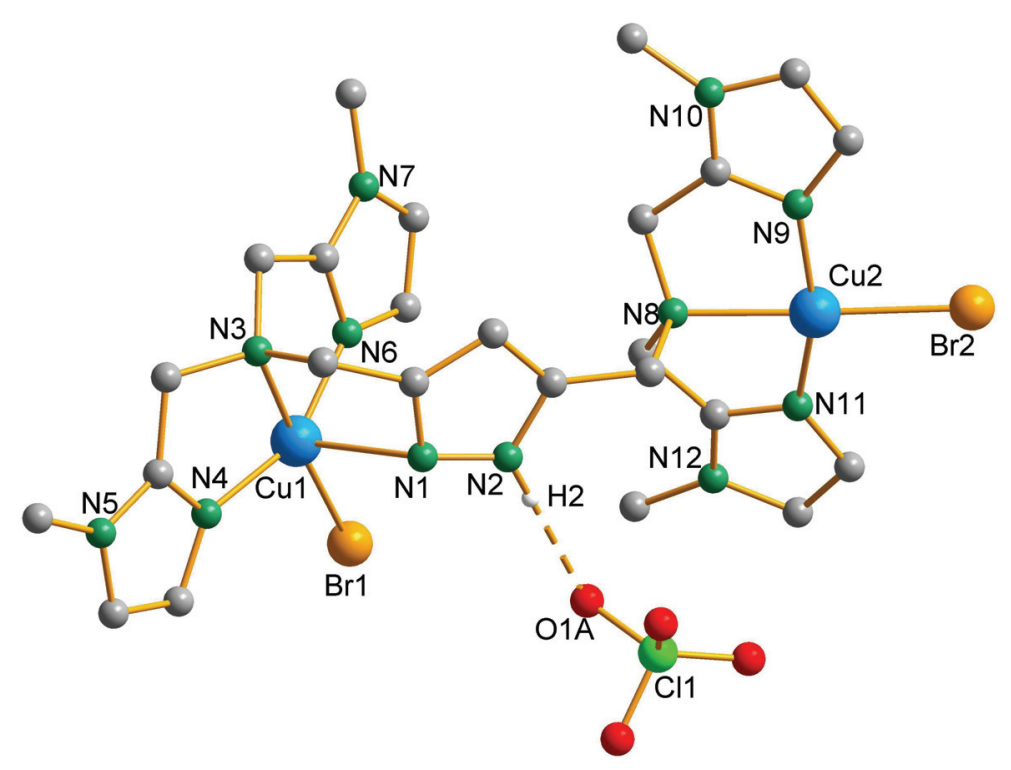

Figure 93. Molecular structure of the cation of complex 17 including one $\mathrm{ClO}_{4}{ }^{-}$anion to indicate the H-bonding with the pyrazole $\mathrm{NH}$-unit. The second one is omitted for clarity.

The molecular structure of complex 17 (Figure 93) revealed the constitution of a structural analogue of 7 , where the pyrazole $\mathrm{N}$-atom is protonated. This protonation induces a splitting of the ( $\mu$-pyrazolato) dicopper core found in 5a and leaves only one of the copper ions coordinated to the now non-bridging pyrazole fragment. Thus, $\mathrm{Cu} 1$ is nested in a distorted square pyramidal coordination environment $(\tau=0.31)$, ligated by four $\mathrm{N}$-donors, including all three nitrogens from the side arm of the ligand, and a Br atom. The second metal is hosted by the dangling chelate arm compartment and is four-coordinated by three N-donors from the side arm and one additional $\mathrm{Br}$ atom resulting in a distorted square planar geometry. Additional H-bonding is observed between the NH-unit of the pyrazole and a perchlorate anion (H2-O1A (2.02 $\AA)$ ). 
Table 21. Selected atom distances $(\AA)$ and angles $\left(^{\circ}\right)$ for complex 17.

\begin{tabular}{llll}
\hline \multicolumn{4}{c}{ Distances } \\
\hline $\mathrm{Cu}(1)-\mathrm{N}(4)$ & $1.923(7)$ & $\mathrm{Cu}(2)-\mathrm{N}(9)$ & $1.895(8)$ \\
$\mathrm{Cu}(1)-\mathrm{N}(6)$ & $1.939(8)$ & $\mathrm{Cu}(2)-\mathrm{N}(11)$ & $1.899(8)$ \\
$\mathrm{Cu}(1)-\mathrm{N}(3)$ & $2.150(8)$ & $\mathrm{Cu}(2)-\mathrm{N}(8)$ & $2.130(6)$ \\
$\mathrm{Cu}(1)-\mathrm{Br}(1)$ & $2.3791(17)$ & $\mathrm{Cu}(2)-\mathrm{Br}(2)$ & $2.3594(13)$ \\
$\mathrm{Cu}(1)-\mathrm{N}(1)$ & $2.382(6)$ & $\mathrm{Cu}(1) \cdots \mathrm{Cu}(2)$ & $8.014(1)$ \\
\hline & & Angles & $80.9(2)$ \\
\hline $\mathrm{N}(4)-\mathrm{Cu}(1)-\mathrm{N}(6)$ & $156.4(3)$ & $\mathrm{N}(3)-\mathrm{Cu}(1)-\mathrm{N}(1)$ & $94.25(18)$ \\
$\mathrm{N}(4)-\mathrm{Cu}(1)-\mathrm{N}(3)$ & $81.3(3)$ & $\mathrm{Br}(1)-\mathrm{Cu}(1)-\mathrm{N}(1)$ & $160.7(4)$ \\
$\mathrm{N}(6)-\mathrm{Cu}(1)-\mathrm{N}(3))$ & $82.4(4$ & $\mathrm{N}(9)-\mathrm{Cu}(2)-\mathrm{N}(11)$ & $82.6(3)$ \\
$\mathrm{N}(4)-\mathrm{Cu}(1)-\mathrm{Br}(1)$ & $99.3(2)$ & $\mathrm{N}(9)-\mathrm{Cu}(2)-\mathrm{N}(8)$ & $81.7(3)$ \\
$\mathrm{N}(6)-\mathrm{Cu}(1)-\mathrm{Br}(1)$ & $98.4(3)$ & $\mathrm{N}(11)-\mathrm{Cu}(2)-\mathrm{N}(8)$ & $97.1(2)$ \\
$\mathrm{N}(3)-\mathrm{Cu}(1)-\mathrm{Br}(1)$ & $175.09(18)$ & $\mathrm{N}(9)-\mathrm{Cu}(2)-\mathrm{Br}(2)$ & $98.0(2)$ \\
$\mathrm{N}(4)-\mathrm{Cu}(1)-\mathrm{N}(1)$ & $104.1(2)$ & $\mathrm{N}(11)-\mathrm{Cu}(2)-\mathrm{Br}(2)$ & $177.66(19)$ \\
$\mathrm{N}(6)-\mathrm{Cu}(1)-\mathrm{N}(1)$ & $90.0(3)$ & $\mathrm{N}(8)-\mathrm{Cu}(2)-\mathrm{Br}(2)$ & \\
\hline
\end{tabular}

Such structural changes in the complex 5a after performing the oxidative C-O coupling of DMBrP are most likely caused by the presence of $\mathrm{HBr}$ that is formed as a by-product. It can be assumed that after coordination of one DMBrP molecule to the dicopper(II) complex 5a, the formation of phenoxyl radicals is taking place and further polymerization proceeds via a radical pathway. In the meantime a lot of studies have shown that $\mathrm{C}-\mathrm{O}$ coupling, in particular of DMP, can also occur via coupling of phenoxonium cations with phenolate anions. ${ }^{179}$ Both mechanistic pathways include release of the H-atom from the para-position in DMP, which in the present case is replaced by a Br-atom. Liberation of $\mathrm{Br}^{-}$, which then binds to the dicopper active site of 5a, led to the structural reorganization, resulting in formation of the complex $\mathbf{1 7}$. No further mechanistic studies were performed on the C-O polymerization of DMBrP. 


\section{Discussion of the proposed mechanism for C-C coupling of TMP}

On the basis of the currently available results, as they are discussed in this thesis, the following mechanism for the oxidative $\mathrm{C}-\mathrm{C}$ coupling of TMP ( $3 \mathrm{e}^{-}$overall per TMP molecule) can be proposed (Scheme 46). The mechanism illustrated below has to be considered only as a preliminary working scheme, as some details are still not totally clear.

\section{Coordination of TMP within the bimetallic pocket of $\mathbf{5 a}$ :}

Coordination of the TMP substrate to the dicopper(II) complex 5a was proven by UV/vis spectroscopy, where an intense band appeared at $\lambda_{\max } 495 \mathrm{~nm}\left(\varepsilon \sim 1500 \mathrm{~L} \cdot \mathrm{mol}^{-1} \cdot \mathrm{cm}^{-1}\right)$ upon the addition of TMP to the solution of 5a. This band was identified as a ligand-to-metal charge-transfer (LMCT) absorption from the phenolate to the copper ion, based on the shift in the UV/vis spectrum to higher wavelength in the order 4-methylphenol (4-MeP), 2,6dimethylphenol (DMP) and 2,4,6-trimethylphenol (TMP), and the characteristic Raman signatures. Such spectroscopic changes were attributed to coordination of a phenolate to one of the copper(II) ions of complex 5a owing to a methanolate-phenolate exchange, concomitant with release of the methanol molecule.

Studies on the determination of the stoichiometry for the reaction of TMP with dicopper complex 5a showed that under aerobic conditions a maximum of two molecules of TMP can coordinate to 5a, resulting in formation of a copper(II)-phenolate species, for which the exact structure is so far unclear. Furthermore, based on identical studies with model substrates such as 4-methylphenol and 4-tert-butylphenol, it was revealed that a key factor required for further reaction to occur is not the number of coordinated phenol substrates, but the substitution pattern and the resulting electronic properties of the phenol ring. In addition, a distinct influence of the steric factors on the further reactivity of the copper(II)-phenolate species was proven. Thus, formation of an unreactive phenolate species was observed with 2,3,5-trimethylphenol (2,3,5-TMP) and 2,5,6-trimethylphenol (2,5,6-TMP) as substrate molecules. This clearly shows that the presence of substituents in the meta-position(s) of the phenol ring is blocking any further reactivity. 


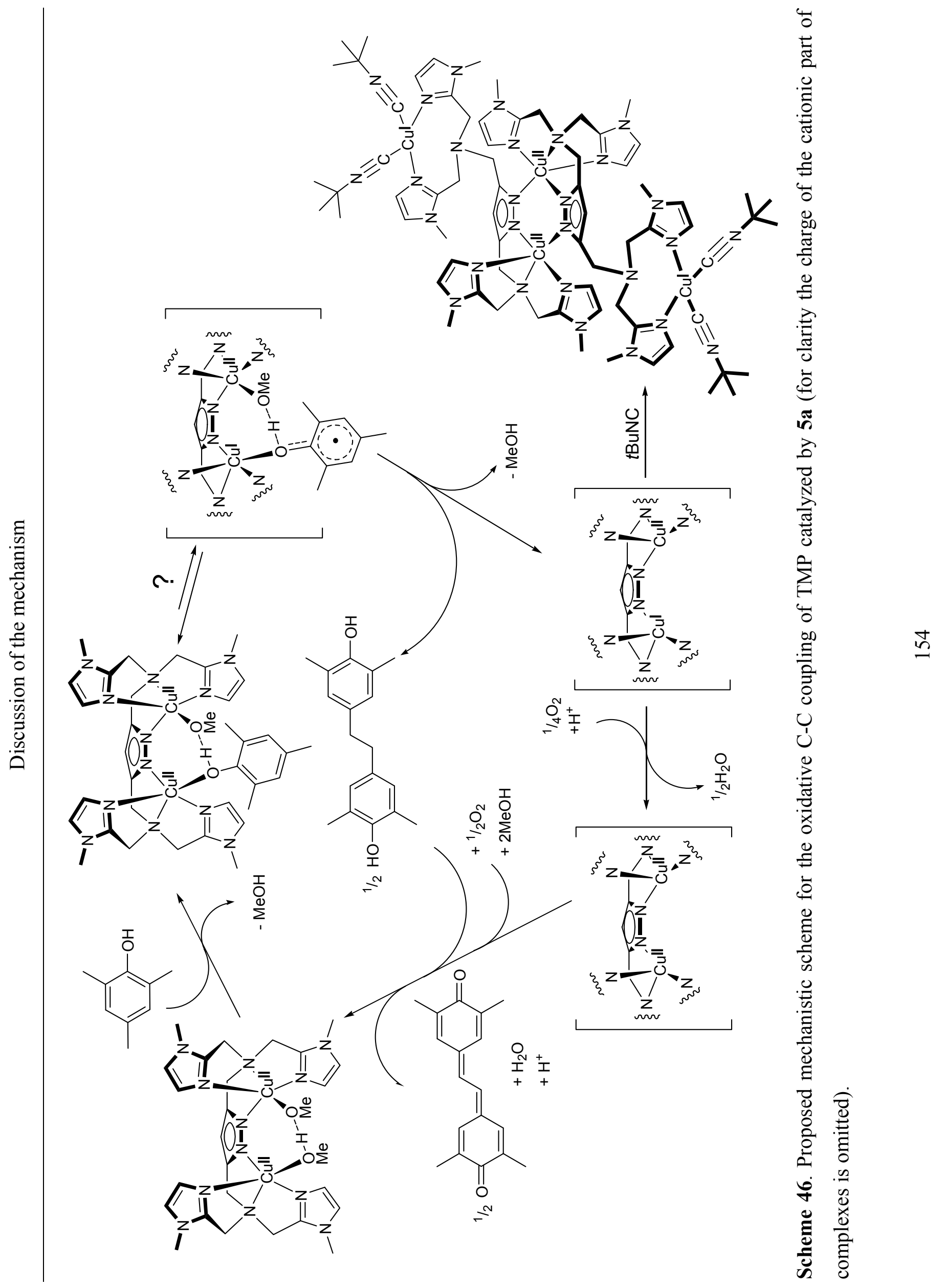




\section{Adducts with model substrate molecules:}

Copper(II)-phenolate complexes with phenolic substrates such as pentafluorophenol (PFP) and 4-hydroxybenzamide (4-AP), that do not display any $\mathrm{Cu}$-mediated reactivity, were fully characterized. Structural features of these model complexes have proven that the assumed methanolate-phenolate exchange occurs upon coordination of phenols and revealed a likely mode of interaction between phenols and dicopper complex 5a. It was shown that coordination of the phenolic molecule occurred only to one of the copper ions in 5a and that the adduct formed is additionally stabilized by $\pi-\pi$ stacking interaction (in case of pentafluorophenol) between the imidazole and phenol rings, as well as by H-bonding between the phenolate-O and a methanol molecule that is coordinated to the adjacent copper ion within the bimetallic pocket.

\section{Active species:}

The nature of the active species formed upon coordination of the TMP substrate within the bimetallic pocket of $\mathbf{5 a}$ is the key point in the mechanism. It is believed that $\mathrm{C}-\mathrm{C}$ coupling of the related substrate DMP occurs via a radical pathway, which would imply the formation of a copper(II)copper(I)-phenoxyl radical species for the studied system with TMP as well. This assumption is not supported by the features observed with UV/vis spectroscopy. Addition of the TMP to 5a induces the appearance of an LMCT band at $495 \mathrm{~nm}$, which is more characteristic for a copper(II)-phenolate species. Resonance Raman spectroscopy was particularly useful and allowed to gain insight into the structural and electronic properties of the species formed. It was shown that interaction of TMP and 5a results in formation of a copper(II)-phenolate species. Typical features for a phenolate coordinated to copper(II) were observed in the Raman spectrum at 1156 ( $v_{9 \mathrm{a}} \mathrm{C}-\mathrm{H}$ bending), 1237 ( $v_{7 \mathrm{a}} \mathrm{C}-\mathrm{O}$ stretching), and $1607 \mathrm{~cm}^{-1}\left(v_{8 \mathrm{a}} \mathrm{C}_{\text {ortho }}-\mathrm{C}_{\text {meta }}\right.$ ring stretching). Moreover, EPR studies on the reaction of a model $\mathrm{Cu}^{\mathrm{II}} \mathrm{Zn}^{\mathrm{II}}$ complex with TMP did not reveal any evidence for the formation of phenoxyl radicals. In this light, it is proposed that the ground state of the adduct can be described as a dicopper(II)-phenolate. However, it can not be excluded that this is in equilibrium with a copper(II)copper(I) phenoxyl radical species (which might also be a thermally accessible excited state species) which is then responsible for further reactions to occur.

Based on studies reported in the literature on the nucleophilic 1,6-addition of small molecules to TMP catalyzed by a mononuclear copper system, ${ }^{99}$ it is believed that one of the possible pathways is formation of a highly active benzoquinone methide intermediate after the first $2 \mathrm{e}^{-}$ oxidation step of TMP. It was proposed that the para-benzylic proton of TMP is activated via 
coordination of the phenolate to the copper center and this $1 \mathrm{e}^{-}$transfer induces the polarization of the benzylic $\mathrm{C}-\mathrm{H}$ bond, resulting in a rearrangement of the aromatic ring to a highly reactive quinone methide. The same kind of 1,6-addition of both $\mathrm{MeOH}$ as well as $d^{4}$ $\mathrm{MeOH}$ to TMP was observed using complex 5a as catalyst. However, there is no evidence for the formation of a putative highly active methide intermediate after initial $2 \mathrm{e}^{-}$oxidation of TMP for the present system, since it would require formation of the fully reduced $\mathrm{Cu}^{\mathrm{I}} \mathrm{Cu}^{\mathrm{I}}$ species, which is not observed experimentally.

\section{Formation of the initial products:}

Formation of the first organic product TMBB in $100 \%$ yield through a stoichiometric reaction (from the 5 equivalents of TMP used only 1 equivalent underwent oxidation) was proven by means of ${ }^{1} \mathrm{H}$ NMR spectroscopy. This process is taking place under both aerobic and anaerobic conditions. However, under an $\mathrm{N}_{2}$-atmosphere, the reaction is halted after the formation of TMBB. The $1 \mathrm{e}^{-}$transfer that occurs upon activation of coordinated TMP implies reduction of only one copper in complex 5a, which was fully confirmed by trapping of the resulting mixed-valence copper species with the strong $\sigma$-donor ligand tert-butyl isocyanide. These results are in good agreement with studies on the stoichiometry between 5a and TMP using Job's method under anaerobic conditions: it was clearly shown that only one TMP molecule is coordinated to the dicopper core and is able to induce a $1 e^{-}$transfer to the $C u^{I I}$ ion it is bound to, resulting in formation of TMBB in a stoichiometric fashion. The formed mixed-valence $\mathrm{Cu}^{\mathrm{I}} \mathrm{Cu}^{\mathrm{II}}$ species after release of the TMBB molecule can not be reoxidized under anaerobic conditions. Presence of the $\mathrm{Cu}^{\mathrm{I}} \mathrm{Cu}^{\mathrm{II}}$ species after this first oxidation step was also proven by means of EPR spectroscopy showing a four-line pattern that is characteristic for a mononuclear copper(II) system.

In marked contrast with these observations, it was discovered that in the presence of dioxygen two molecules of TMP were found to interact with copper complex 5a, which is most likely the result of several parallel processes: under aerobic conditions the mixed-valence $C u^{I} C u^{I I}$ species formed after release of TMBB is reoxidized and able to bind either another TMP molecule or the TMBB intermediate, to finally form the product TMSQ. In this light, the Job plot of TMP with $\mathbf{5 a}$ under aerobic conditions represents a mixture of different species formed during the overall reaction, resulting in the average amount of two coordinated TMP molecules. 
The stoichiometrically formed TMBB can be further oxidized in air only in the presence of the parent dicopper complex 5a. Therefore aerial dioxygen is required for the reoxidation of the mixed-valence $\mathrm{Cu}^{\mathrm{I}} \mathrm{Cu}^{\mathrm{II}}$ species formed after the first reaction step.

The $4 \mathrm{e}^{-}$oxidation reaction of TMBB to yield TMSQ most likely occurs via formation of a second organic intermediate, concurrent with generation of another mixed-valence $\mathrm{Cu}^{\mathrm{I}} \mathrm{Cu}^{\mathrm{II}}$ species. Unfortunately, detection of the second organic intermediate was not possible under both aerobic (formation in non-isolated yield) and anaerobic conditions (rapid formation of TMSQ from TMBB).

$\mathrm{H}_{2} \mathrm{O}_{2}$ test:

It was convincingly shown that $\mathrm{H}_{2} \mathrm{O}_{2}$ is not formed during the oxidative $\mathrm{C}-\mathrm{C}$ coupling of TMP to TMSQ. This means that water is formed as the reduction product, implying that 1.5 molar equivalents of $\mathrm{O}_{2}$ is needed per molecule of TMSQ formed. 


\section{Conclusions}

In the present thesis the design-concept for pyrazole-based ligands, which makes use of the fact that the anionic pyrazolate unit has a high tendency to span two metal ions in a $\mu_{-}-\eta^{1}: \eta^{1}$ exo-bidentate fashion, was applied for a range of copper complexes and moreover, the coordination spheres of the individual copper ions as well as the intramolecular metal-metal separation were properly tuned. Ligand $\mathbf{H L}^{2}$ with $N$-dimethyl-aminopropyl groups was shown to undergo an unusual copper mediated amine to $N$-oxide transformation. The formed copper complexes with bound $N$-oxide were structurally characterized. On the basis of selected performed studies, it was concluded that this kind of amine to $\mathrm{N}$-oxide transformation is taking place only during the crystallization procedure and in the presence of aerial dioxygen. No formation of $\mathrm{N}$-oxide species was observed under anaerobic conditions.

Furthermore, new pyrazole-based ligands with bio-inspired bis[(1-methylimidazole-2-yl)methyl]amine chelating side arms in the 3- and 5-positions of the heterocyclic core were prepared and fully characterized. The coordination behaviour of these new ligands $\left(\mathbf{H L}^{4}\right.$ and $\mathbf{H L}^{\mathbf{5}}$ ) towards copper was elucidated and it was shown that these ligands form very stable copper complexes. Determination of the different species formed in solution, depending on the $\mathrm{pH}$ (in particular for complexes based on a ligand with appended bis[2-(1methylimidazolyl)methyl]aminomethyl chelate arms) was successfully performed by potentiometric and spectroscopic methods and all relevant copper complexes were structurally characterized.

Properly characterized copper complexes, in particular 5a, with carefully tuned and desired properties, were applied as catalysts in different types of oxidation reactions of phenolic substrates. In particular, it was shown that dicopper complex 5a catalyzes the unusual oxidative C-C coupling of 2,4,6-trimethylphenol (TMP) to yield TMSQ. Moreover, the formation and structure of intermediates in the catalytic reactions of TMP were elucidated by means of various spectroscopic methods (i.e. Raman, EPR, NMR and UV/vis spectroscopy). On the basis of these studies it was shown that C-C coupling of TMP is taking place only in the presence of dicopper complex $\mathbf{5 a}$ and dioxygen as oxidant. The mixed-valence $\mathrm{Cu}^{\mathrm{I}} \mathrm{Cu}^{\mathrm{II}}$ species which is formed after the first oxidation step of TMP was trapped using the strong $\sigma$ donor isocyanide ligand and structurally characterized. Furthermore, the first organic 
intermediate formed during the catalytic C-C coupling reaction - TMBB - was isolated. It was clearly shown that further oxidation of this biphenol intermediate is possible only in the presence of initial dicopper complex 5a and therefore, aerial dioxygen is required for the oxidation of the mixed-valence $\mathrm{Cu}^{\mathrm{I}} \mathrm{Cu}^{\mathrm{II}}$ species formed after the first reaction step. Dicopper(II)-phenolate complexes with 4-hydroxybenzamide and pentafluorophenol substrates that were considered inert to any oxidation reaction were successfully prepared, which revealed a mode of interaction between phenols and dicopper complex 5a. It was shown that coordination of the phenolic molecule occurred only to one of the copper ions in 5a and that the adduct formed is additionally stabilized by $\pi-\pi$ stacking interaction (in case of pentafluorophenol) between the imidazole and phenol rings as well as by H-bonding between the phenolate-O and methanol molecule that is coordinated to the adjacent copper ion within the bimetallic pocket.

The ground state of the key species in the C-C coupling reaction of TMP was characterized as a dicopper(II)-phenolate, which was proven by means of Raman and EPR spectroscopy.

All these above-mentioned studies provided a better understanding of the principles/factors that govern the catalytic oxidation activity towards phenols of pyrazolate-based complexes reminiscent of type 3 dicopper sites, and allowed to propose a preliminary mechanism for the oxidative C-C coupling of TMP.

In addition, it was shown that, depending on the reaction conditions applied, dicopper complex 5a can catalyze the 1,6-nucleophilic addition of several small molecules such as $\mathrm{MeOH}$ to TMP. The observed 1,6-addition of $\mathrm{MeOH}$ and $d^{4}-\mathrm{MeOH}$ catalyzed by dicopper complex 5a showed the significant potential of this complex to act as a versatile catalyst in different kinds of phenol oxidations besides the $\mathrm{C}-\mathrm{C}$ coupling reaction. This can be used in the future to generate new organic compounds, not only by means of $\mathrm{C}-\mathrm{C}$ bond formation but in particular also by $\mathrm{C}-\mathrm{N}$ and $\mathrm{C}-\mathrm{O}$ bond making reactions.

Furthermore, a C-O bond forming reaction with 4-bromo-2,6-dimetylphenol was shown to occur in the presence of $\mathbf{5 a}$ as catalyst to yield a polymeric phenylene ether.

Finally, ortho C-C coupling of 4-ethylphenol to yield an oligomeric compound can be also performed using an analogue to copper complex 5a based on a pyrazole ligand $\left(\mathbf{H L}^{\mathbf{5}}\right)$ with a modified backbone. An oligonuclear copper complex 15 that represent an intermediate in the described reaction was crystallized and structurally characterized. 
All these studies have shown the vital potential of a new class of bioinspired copper complexes, based on biorelevant pyrazole ligands, to act as catalysts in various oxidation reactions of phenols. Moreover, this work presents mechanistic insight, in particular on the unusual oxidative C-C coupling of TMP to yield TMSQ. These results will undoubtedly provide the significant background necessary to advance future studies on bioinspired phenol oxidation reactions.

Every time we set ourselves to learn, we have to labor as hard as anyone can. The limits of our learning will be determined by our nature.

The Wheel of Time

Carlos Castaneda 


\section{Experimental section}

\subsection{General}

Equipment: Preparations of air sensitive materials were carried out under nitrogen atmosphere using standard Schlenk techniques or in the $\mathrm{N}_{2}$-filled glove box. All glassware was dried by heating under vacuum.

Solvent purification: Solvents were purified and dried by established procedures, in particular: Aceton (calcium hydride), Acetonitrile (phosphorus pentoxide), Dichloromethane (calcium hydride), Diethylether (sodium/potassium), Tetrahydrofuran (sodium/potassium), Ethanol (sodium), Methanol (magnesium).

Elemental analysis: Microanalyses were performed by the Analytisches Labor des Anorganisch-Chemischen Instituts der Universität Göttingen using a CHN-O-Rapid from the company Elementar.

Infrared spectroscopy: IR spectra (as $\mathrm{KBr}$ pellets) were recorded with a Digilab Excalibur. The given intensity in the experimental section is abbreviated as follows:

$\begin{array}{ll}\text { vs } & \text { very strong } \\ \text { s } & \text { strong } \\ \text { m } & \text { medium } \\ \text { w } & \text { weak }\end{array}$

UV/vis spectroscopy: UV/vis spectra of solutions and solids (diffuse reflectance) were recorded with a Varian Cary 5000 spectrometer at room temperature using quartz cuvettes (L $=1 \mathrm{~cm})$.

Mass spectrometry: Mass spectra were measured with a Finnigan MAT 95 (FAB-MS), a Finnigan MAT LCQ (HR-ESI-MS) and with an Applied Biosystems API 2000 (ESI-MS). For the FAB-MS measurement 3-nitrobenzylalcohol (3-NBA) or glycerine were used as matrix. EI spectra were recorded with a Finnigan MAT $8200(70 \mathrm{eV})$. 
NMR: NMR spectra were recorded with a Bruker Avance $200\left({ }^{1} \mathrm{H}: 200.13 \mathrm{MHz},{ }^{13} \mathrm{C}: 50.3\right.$ $\mathrm{MHz}), 500\left({ }^{1} \mathrm{H}: 500.13 \mathrm{MHz},{ }^{13} \mathrm{C}: 125.7 \mathrm{MHz}\right)$ and with a Bruker $900 \mathrm{MHz}$ NMRSpektrometer $(52 \mathrm{~mm})$ at room temperature. The residual solvent signal was used as the chemical shift reference $\left(\mathrm{CDCl}_{3}: \delta_{\mathrm{H}}=7.24, \delta_{\mathrm{C}}=77.0\right),\left(\left(\mathrm{CD}_{3}\right)_{2} \mathrm{SO}: \delta_{\mathrm{H}}=2.54, \delta_{\mathrm{C}}=40.45\right)$ and $\left(\mathrm{CD}_{3} \mathrm{CN}: \delta_{\mathrm{H}}=1.96, \delta_{\mathrm{C}}=118.26\right)$ relative to external trimethylsilane $(\delta=0)$. Abbreviated as follows: s (singlet), d (doublet), t (triplet), m (multiplet).

Magnetic measurements: Magnetic data were measured with a Quantum-Design MPMS-5S SQUID magnetometer equipped with a 5 Tesla magnet in the range from $295 \mathrm{~K}$ to $2 \mathrm{~K}$. The powdered samples were contained in a gel bucket and fixed in a non-magnetic sample holder.

Stopped-Flow measurement: Stopped-Flow measurements were performed using Bio-Logic stopped-flow module SFM-20 with gas tight glass syringers Hamilton 1010C at room temperature.

Raman spectroscopy: Resonance Raman spectra were obtained using LabRAM HR Vis (400$1100 \mathrm{~nm}$ ) with open-electrode CCD detector. Excitation was provided by an $\mathrm{Ar}^{+}$ion laser. The laser line, $488.7 \mathrm{~nm}$, was chosen to coincide with the intense absorption transition of the copper(II)-phenolate species. Sample concentrations were approximately 5-6 $\mathrm{mM}$ in dicopper complexes 5a or 8. All measurements were carried out at room temperature in $\mathrm{MeCN}$.

GC-MS measurement: GC-MS spectra were recorded using Finnigan INCOS 50 with connected gas chromatograph Varian 3400.

Cyclovoltammetry: Redox properties have been examined by cyclovoltammetry in MeCN/0.1 $\mathrm{M}\left[\mathrm{NBu}_{4}\right] \mathrm{PF}_{6}$ at room temperature. A glassy carbon electrode was used as a working electrode, platinum as a counter electrode and SCE (Ag-electrode) as reference electrode. All spectra were measured versus the $\mathrm{Cp}_{2} \mathrm{Fe} / \mathrm{Cp}_{2} \mathrm{Fe}^{+}$couple.

EPR measurements: X-band EPR were measured on a Bruker ELEXSYS E 500 spectrometer, equipped with a digital temperature control ER 4131 VT using helium as coolant.

\section{Chemicals:}

3,5-Bis(chloromethyl)-1-(tetrahydropyran-2-yl)pyrazole ${ }^{100,101,102}$ 
Bis[(6-methyl-2-pyridyl)methyl]amine XXIX, ${ }^{103 a}$

Bis[(1-methylimidazol-2-yl)-methyl $]$ amine ${ }^{103 b, c}$

3,5-Bis(hydroxymethyl)-4-phenyl-pyrazole ${ }^{104}$

$C D_{3}-\mathrm{TMP}^{130}$

All other chemicals were purchased from commercial sources (Merck, Aldrich, Lancaster and Grüssing) and used as received.

Caution! Although no problems were encountered in this work, transition metal perchlorate complexes are potentially explosive and should be handled with proper precautions. 


\subsection{Synthesis of ligands $\mathrm{HL}^{1}-\mathrm{HL}^{5}$}

Ligands $\mathbf{H L}^{\mathbf{1}}$ and $\mathbf{H L}^{\mathbf{2}}$ were prepared by modified literature procedure ${ }^{15 \mathrm{a}}$

\section{ligand $\mathrm{HL}^{1}$ :}
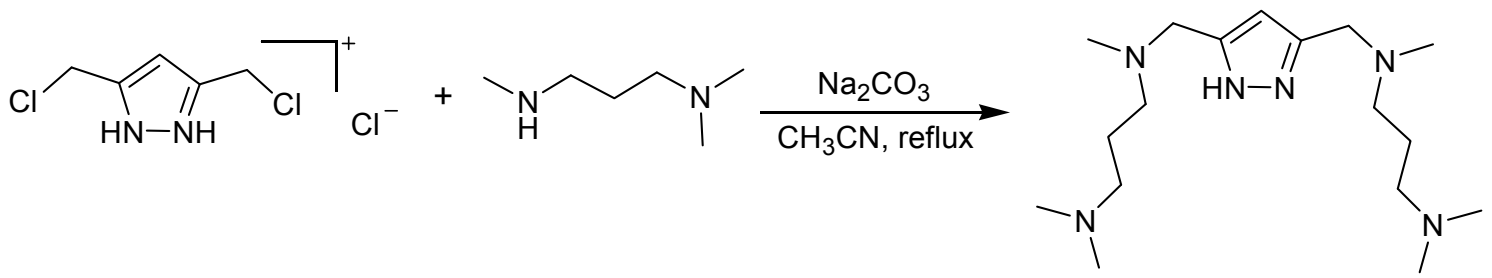

3,5-Bis(chloromethyl)pyrazole hydrochloride (4.02 g, $20 \mathrm{mmol})$ and $N, N, N$ '-trimethyl-1,3propanediamine $(5.0 \mathrm{~g}, 40 \mathrm{mmol})$ were dissolved in $600 \mathrm{~mL}$ of $\mathrm{MeCN}$ and $\mathrm{Na}_{2} \mathrm{CO}_{3}(24.38 \mathrm{~g}$, $0.23 \mathrm{~mol}$, pre-dried at $100-120^{\circ} \mathrm{C}$ and $10^{-3} \mathrm{mbar}$ ) was added to this solution. The reaction mixture was refluxed for 4 days while stirring. After filtration to remove insolubles, the solvent was evaporated and the residue was dried at $60^{\circ} \mathrm{C}$ and $10^{-3}$ mbar for $12 \mathrm{~h}$. $\mathbf{H L}^{1}$ was obtained as light yellow oil.

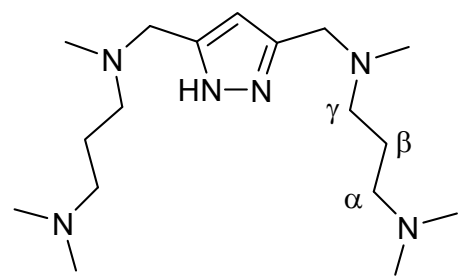

Yield:

$3.95 \mathrm{~g}, 61 \%$

Empirical formula:

$\mathrm{C}_{17} \mathrm{H}_{36} \mathrm{~N}_{6}$

Molecular weight:

$324.5 \mathrm{~g} / \mathrm{mol}$

${ }^{1} \mathrm{H}-\mathrm{NMR}\left(\mathrm{CDCl}_{3}\right) \delta(\mathrm{ppm})$ :

$1.32\left(\mathrm{p}, J=7.2 \mathrm{~Hz} \mathrm{CH}_{2}(\beta), 4 \mathrm{H}\right), 1.87\left(\mathrm{~s}, \mathrm{CH}_{3}, 12 \mathrm{H}\right)$, $1.88\left(\mathrm{~s}, \mathrm{CH}_{3}, 6 \mathrm{H}\right), 1.96\left(\mathrm{t}, J=7.2 \mathrm{~Hz}, \mathrm{CH}_{2}(\alpha), 4 \mathrm{H}\right), 2.05$ (t, $J=$ 
$\left.7.2 \mathrm{~Hz}, \mathrm{CH}_{2}(\gamma), 4 \mathrm{H}\right), 3.21$ (s, pz-CH, $\left.4 \mathrm{H}\right), 5.68$ (s, pz-CH, 1H)

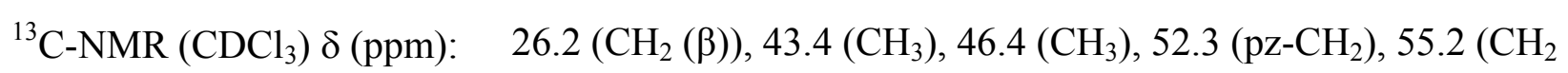
$(\alpha)), 55.8\left(\mathrm{CH}_{2}(\gamma)\right), 104.9\left(\mathrm{pz}-\mathrm{C}^{4}\right), \mathrm{pz}-\mathrm{C}^{3 / 5}$ not observed

MS (EI) $m / z(\%)$ :

$324(35)\left[\mathrm{C}_{17} \mathrm{H}_{36} \mathrm{~N}_{6}\right]^{+}$

238 (15) $\left[\mathrm{C}_{17} \mathrm{H}_{36} \mathrm{~N}_{6}-\left(\mathrm{CH}_{2}\right)_{3} \mathrm{~N}\left(\mathrm{CH}_{3}\right)_{2}\right]^{+}$

$210(50)\left[\mathrm{C}_{17} \mathrm{H}_{36} \mathrm{~N}_{6}-\mathrm{N}\left(\mathrm{CH}_{3}\right)\left(\mathrm{CH}_{2}\right)_{3} \mathrm{~N}\left(\mathrm{CH}_{3}\right)_{2}\right]^{+}$

Elemental analysis (\%): $\quad$ for $\mathrm{C}_{17} \mathrm{H}_{36} \mathrm{~N}_{6}$

calcd.: C 62.92, H 11.18, N 25.90

found: C 61.12, H 11.28, N 25.77

ligand $\mathrm{HL}^{2}$ :

(lin

3,5-Bis(chloromethyl)pyrazole hydrochloride (4.02 g, $20 \mathrm{mmol})$ and N,N-bis-(3dimethylaminopropyl)amine $(7.48 \mathrm{~g}, 40 \mathrm{mmol})$ were dissolved in $700 \mathrm{~mL}$ of $\mathrm{MeCN}$ and $\mathrm{Na}_{2} \mathrm{CO}_{3}\left(24.38 \mathrm{~g}, 0.23 \mathrm{~mol}\right.$, pre-dried at $100-120^{\circ} \mathrm{C}$ and $\left.10^{-3} \mathrm{mbar}\right)$ was added to this solution. The reaction mixture was refluxed for 3 days while stirring. After filtration, the solvent was evaporated. Unreacted starting amine was separated from the desired ligand by high vacuum distillation $\left(50^{\circ} \mathrm{C}\right.$ and $\left.10^{-3}\right)$ mbar and the residue obtained was dried at $60^{\circ} \mathrm{C}$ and $10^{-3}$ mbar for $12 \mathrm{~h} . \mathbf{H L}^{2}$ was obtained as light yellow oil.

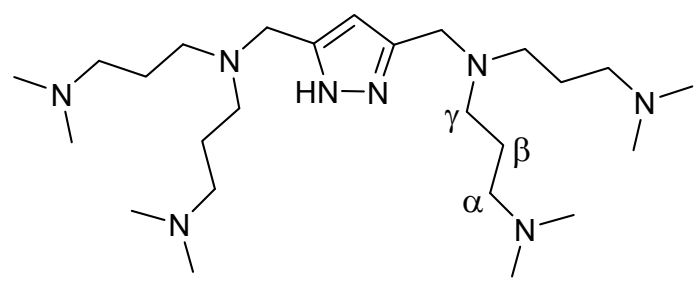


Yield:

Empirical formula:

Molecular weight:

${ }^{1} \mathrm{H}-\mathrm{NMR}\left(\mathrm{CDCl}_{3}\right) \delta(\mathrm{ppm})$ :

${ }^{13} \mathrm{C}-\mathrm{NMR}\left(\mathrm{CDCl}_{3}\right) \delta(\mathrm{ppm})$ :

MS (EI) $m / z(\%)$

Elemental analysis (\%):
$5.59 \mathrm{~g}, 60 \%$

$\mathrm{C}_{25} \mathrm{H}_{54} \mathrm{~N}_{8}$

$466.5 \mathrm{~g} / \mathrm{mol}$

1.48 (p, $\left.J=7.1 \mathrm{~Hz} \mathrm{CH}_{2}(\beta), 8 \mathrm{H}\right), 2.04$ (s, $\left.\mathrm{CH}_{3}, 24 \mathrm{H}\right)$,

$2.12\left(\mathrm{t}, J=7.1 \mathrm{~Hz}, \mathrm{CH}_{2}(\alpha), 8 \mathrm{H}\right), 2.31\left(\mathrm{t}, J=7.1 \mathrm{~Hz}, \mathrm{CH}_{2}(\gamma)\right.$,

8H), 3.47 (s, pz-CH $\mathrm{CH}_{2}, 4 \mathrm{H}$ ), 5.77 (s, pz-CH, 1H)

$24.6\left(\mathrm{CH}_{2}(\beta)\right), 44.8\left(\mathrm{CH}_{3}\right), 44.9\left(\mathrm{CH}_{3}\right), 50.0\left(\mathrm{pz}^{\left.-\mathrm{CH}_{2}\right)}, 51.2\left(\mathrm{CH}_{2}\right.\right.$

$(\alpha)), 57.1\left(\mathrm{CH}_{2}(\gamma)\right), 102.2\left(\mathrm{pz}-\mathrm{C}^{4}\right), \mathrm{pz}^{-\mathrm{C}^{3 / 5}}$ not observed

$466(20)\left[\mathrm{C}_{25} \mathrm{H}_{54} \mathrm{~N}_{8}\right]^{+}$

$380(5)\left[\mathrm{C}_{25} \mathrm{H}_{54} \mathrm{~N}_{8}-\left(\mathrm{CH}_{2}\right)_{3} \mathrm{~N}\left(\mathrm{CH}_{3}\right)_{2}\right]^{+}$

$281(40)\left[\mathrm{C}_{25} \mathrm{H}_{54} \mathrm{~N}_{8}-\left\{\mathrm{N}\left(\mathrm{CH}_{3}\right)\left(\mathrm{CH}_{2}\right)_{3} \mathrm{~N}\left(\mathrm{CH}_{3}\right)_{2}\right\}_{2}\right]^{+}$

for $\mathrm{C}_{25} \mathrm{H}_{54} \mathrm{~N}_{8} \cdot 2 \mathrm{H}_{2} \mathrm{O}$

calcd.: C 59.72, H 11.63, N 22.29

found: C 60.27, H 11.76, N 22.91

\section{ligand $\mathrm{HL}^{3}$ :}
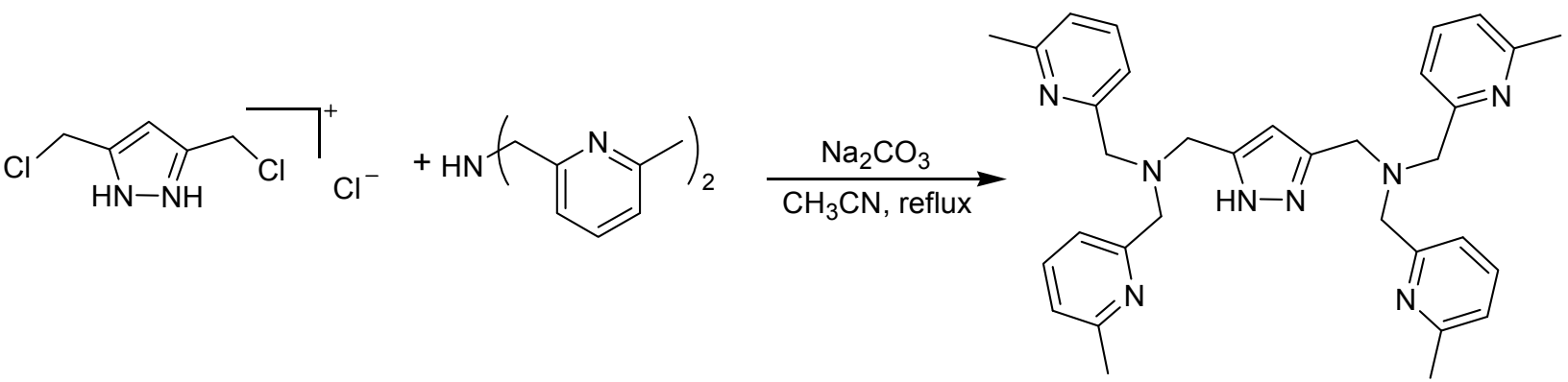

$N, N$-bis[(6-methyl-pyridin-2-yl)methyl]amine $\quad(5.33 \quad \mathrm{~g}, \quad 20 \quad \mathrm{mmol}) \quad$ and 3,5bis(chloromethyl)pyrazole hydrochloride $(2.01 \mathrm{~g}, 10 \mathrm{mmol})$ were dissolved in $500 \mathrm{~mL}$ of $\mathrm{MeCN}$ and $\mathrm{Na}_{2} \mathrm{CO}_{3}\left(12.5 \mathrm{~g}, 0.12 \mathrm{~mol}\right.$, pre-dried at $100-120^{\circ} \mathrm{C}$ and $10^{-3} \mathrm{mbar}$ ) was added to this solution. The reaction mixture was refluxed for $24 \mathrm{~h}$ while stirring. After filtration, the solvent was evaporated and the residue was dried at $60^{\circ} \mathrm{C}$ and $10^{-5}$ mbar for $12 \mathrm{~h}$. $\mathbf{H L}^{3}$ was obtained as a white solid. 
Yield:

$3.9 \mathrm{~g}, 72 \%$

Empirical formula: $\quad \mathrm{C}_{33} \mathrm{H}_{38} \mathrm{~N}_{8}$

Molecular weight: $\quad 546.3 \mathrm{~g} / \mathrm{mol}$

Melting point: $\quad 110-112^{\circ} \mathrm{C}$

${ }^{1} \mathrm{H}-\mathrm{NMR}\left(\mathrm{CD}_{3} \mathrm{OD}\right) \delta(\mathrm{ppm}): \quad 2.45\left(\mathrm{~s}, \mathrm{CH}_{3}, 12 \mathrm{H}\right), 3.72\left(\mathrm{~s}, \mathrm{pz}-\mathrm{CH}_{2}, 4 \mathrm{H} ; \mathrm{NCH}_{2}, 8 \mathrm{H}\right), 6.31$ (s, pz$\mathrm{CH}, 1 \mathrm{H}), 7.07$ (d, $J=7.6 \mathrm{~Hz}$, py-CH, 4H), 7.40 (d, $J=7.7 \mathrm{~Hz}$, py$\mathrm{CH}, 4 \mathrm{H}), 7.62$ (t, $J=7.7 \mathrm{~Hz}$, py-CH, 4H)

${ }^{13} \mathrm{C}-\mathrm{NMR}\left(\mathrm{CD}_{3} \mathrm{OD}\right) \delta(\mathrm{ppm}): 23.7\left(\mathrm{CH}_{3}\right), 60.69\left(\mathrm{CH}_{2}\right), 106.1\left(\mathrm{pz}-\mathrm{C}^{4}\right), 121.5$ (py-C), 123.2 (pyC), 138.80 (py-C), 158.67 (py-C), 157.76 (py-C), pz-C ${ }^{3 / 5}$ not observed

MS (EI) $m / z(\%): \quad 546(10)\left[\mathrm{C}_{33} \mathrm{H}_{38} \mathrm{~N}_{8}\right]^{+}$ $440(100)\left[\mathrm{C}_{33} \mathrm{H}_{38} \mathrm{~N}_{8}-\left(\mathrm{CH}_{2}\right) \mathrm{C}_{5} \mathrm{H}_{3} \mathrm{~N}\left(\mathrm{CH}_{3}\right)\right]^{+}$

Elemental analysis (\%): $\quad$ for $\mathrm{C}_{33} \mathrm{H}_{38} \mathrm{~N}_{8} \cdot \mathrm{H}_{2} \mathrm{O}$ calcd.: C 70.19, H 7.14, N 19.84 found: C 70.03, H 7.12, N 19.56

ligand $\mathrm{HL}^{4}$ :



$N, N$-Bis[2-(1-methylimidazolyl)methyl]amine $\quad(20.50 \quad$ g, $\quad 100 \quad \mathrm{mmol}) \quad$ and 3,5bis(chloromethyl)pyrazole hydrochloride $(10.25 \mathrm{~g}, 50 \mathrm{mmol})$ were dissolved in $700 \mathrm{~mL}$ of $\mathrm{MeCN}$ and $\mathrm{Na}_{2} \mathrm{CO}_{3}\left(53 \mathrm{~g}, 0.50 \mathrm{~mol}\right.$, pre-dried at $100-120^{\circ} \mathrm{C}$ and $10^{-3} \mathrm{mbar}$ ) was added to this solution. The reaction mixture was refluxed for $24 \mathrm{~h}$ while stirring. After filtration, the solvent was evaporated and the residue was dried at $60^{\circ} \mathrm{C}$ and $10^{-3} \mathrm{mbar}$ for $12 \mathrm{~h}$. $\mathbf{H L}^{4}$ was obtained as a light yellow solid. 
Yield:

Empirical formula:

Molecular weight:

Melting point:

${ }^{1} \mathrm{H}-\mathrm{NMR}\left(\mathrm{CDCl}_{3}\right) \delta(\mathrm{ppm})$ :

$20.80 \mathrm{~g}, 83 \%$

$\mathrm{C}_{25} \mathrm{H}_{34} \mathrm{~N}_{12}$

$502.3 \mathrm{~g} / \mathrm{mol}$

$78-80^{\circ} \mathrm{C}$

$3.45\left(\mathrm{~s}, \mathrm{CH}_{3}, 12 \mathrm{H}\right), 3.57\left(\mathrm{~s}, \mathrm{pzCH}_{2}, 4 \mathrm{H}\right), 3.62\left(\mathrm{~s}, \mathrm{NCH}_{2}, 8 \mathrm{H}\right)$,

6.14 (s, pz-CH, 1H), 6.79 (d, $J=1 \mathrm{~Hz}, \mathrm{im}-\mathrm{CH}, 4 \mathrm{H}), 6.91$ (d, $J=1$

$\mathrm{Hz}, \mathrm{im}-\mathrm{CH}, 4 \mathrm{H})$

${ }^{13} \mathrm{C}-\mathrm{NMR}\left(\mathrm{CDCl}_{3}\right) \delta(\mathrm{ppm}): \quad 32.4\left(\mathrm{CH}_{3}\right), 48.6\left(\mathrm{CH}_{2}\right), 49.0\left(\mathrm{CH}_{2}\right), 106.7\left(\mathrm{pz}-\mathrm{C}^{4}\right), 121.4(\mathrm{im}-$ C), 127.0 (im-C), 145.0 (im- $\left.\mathrm{C}^{2}\right), \mathrm{pz}-\mathrm{C}^{3 / 5}$ not observed

MS (EI) $m / z(\%)$ :

$502(5)\left[\mathrm{C}_{25} \mathrm{H}_{34} \mathrm{~N}_{12}\right]^{+}$

407 (100) $\left[\mathrm{C}_{25} \mathrm{H}_{34} \mathrm{~N}_{12}-\left(\mathrm{CH}_{2}\right) \mathrm{C}_{3} \mathrm{H}_{2} \mathrm{~N}_{2}\left(\mathrm{CH}_{3}\right)\right]^{+}$

Elemental analysis (\%): $\quad$ for $\mathrm{C}_{25} \mathrm{H}_{34} \mathrm{~N}_{12} \cdot \mathrm{H}_{2} \mathrm{O}$

calcd.: C 57.67, H 6.58, N 32.28

found: C 57.88, H 6.81, N 32.78

ligand $\mathrm{HL}^{5}$ :

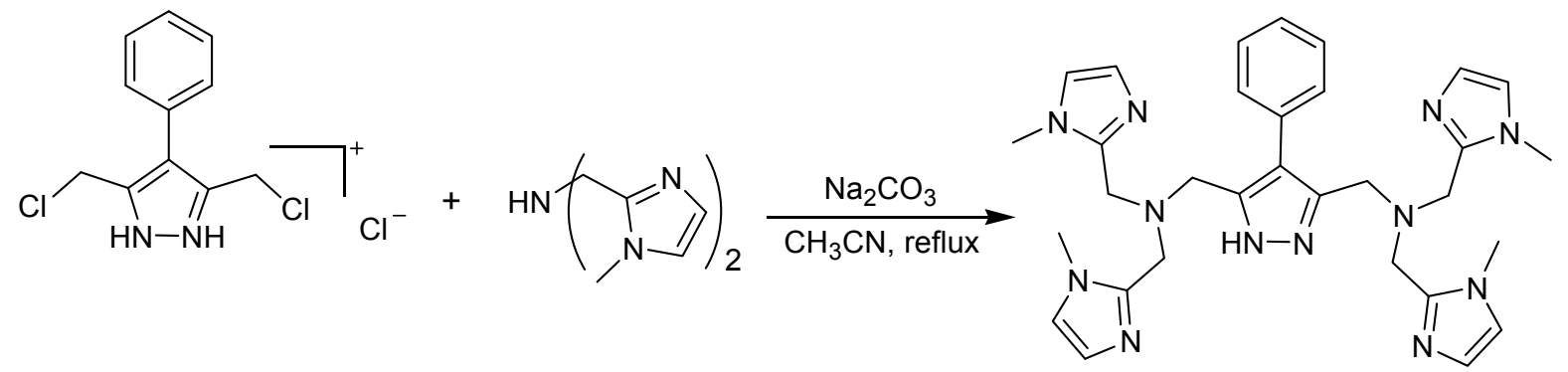

3,5-Bis(chloromethyl)-4-phenylpyrazole hydrochloride (1.80 g, $6 \mathrm{mmol})$ and bis[2-(1methylimidazolyl)methyl]amine (2.46 g, $12 \mathrm{mmol})$ were dissolved in $100 \mathrm{~mL}$ of $\mathrm{MeCN}$ and $\mathrm{Na}_{2} \mathrm{CO}_{3}\left(6.36 \mathrm{~g}, 60 \mathrm{mmol}\right.$, pre-dried at $100-120^{\circ} \mathrm{C}$ and $\left.10^{-3} \mathrm{mbar}\right)$ was added to this solution. The reaction mixture was refluxed for $48 \mathrm{~h}$ while stirring. After filtration, the solvent was evaporated and the residue was dried at $60^{\circ} \mathrm{C}$ and $10^{-3}$ mbar for $12 \mathrm{~h}$. $\mathbf{H L}^{\mathbf{5}}$ was obtained as a light yellow solid. 
Yield:

Empirical formula:

Molecular weight:

Melting point:

${ }^{1} \mathrm{H}-\mathrm{NMR}\left(\mathrm{CDCl}_{3}\right) \delta(\mathrm{ppm})$ :$$
6.69(\mathrm{~d}, J=1 \mathrm{~Hz}, \mathrm{im}-\mathrm{CH}, 4 \mathrm{H}), 6.871(\mathrm{~d}, J=1 \mathrm{~Hz}, \mathrm{im}-\mathrm{CH}, 4 \mathrm{H}) \text {, }
$$$$
7.0 \text { (m, Ar-CH, 2H), } 7.16 \text { (m, Ar-CH, 3H) }
$$

${ }^{13} \mathrm{C}-\mathrm{NMR}\left(\mathrm{CDCl}_{3}\right) \delta(\mathrm{ppm}): \quad 32.2\left(\mathrm{CH}_{3}\right), 48.3\left(\mathrm{CH}_{2}\right), 49.0\left(\mathrm{CH}_{2}\right), 120.2\left(\mathrm{pz}-\mathrm{C}^{4}\right), 121.4(\mathrm{im}-$ C), 126.0 (Ar-CH), 127.0 (im-C), 128.4 (Ar-CH), 128.8 (Ar-CH), 133.3 (Ar-CH), 145.0 (im- $\left.\mathrm{C}^{2}\right), \mathrm{Ar}-\mathrm{C}^{6}$ not observed, pz- $\mathrm{C}^{3 / 5}$ not observed

MS (EI) $m / z(\%)$ :

$578(5)\left[\mathrm{C}_{31} \mathrm{H}_{38} \mathrm{~N}_{12}\right]^{+}$

483 (30) $\left[\mathrm{C}_{31} \mathrm{H}_{38} \mathrm{~N}_{12}-\left(\mathrm{CH}_{2}\right) \mathrm{C}_{3} \mathrm{H}_{2} \mathrm{~N}_{2}\left(\mathrm{CH}_{3}\right)\right]^{+}$

Elemental analysis (\%): for $\mathrm{C}_{31} \mathrm{H}_{38} \mathrm{~N}_{12} \cdot 3 \mathrm{H}_{2} \mathrm{O}$

calcd.: C 58.84, H 7.01, N 26.56

found: C 58.70, H 7.00, N 26.40 


\subsection{Synthesis of complexes}

Preparation of $\left[\mathrm{Cu}_{2} \mathrm{~L}^{1}(\mu-\mathrm{OH})(\mathrm{MeOH})_{2}\right]\left(\mathrm{BF}_{4}\right)_{2}(1)$

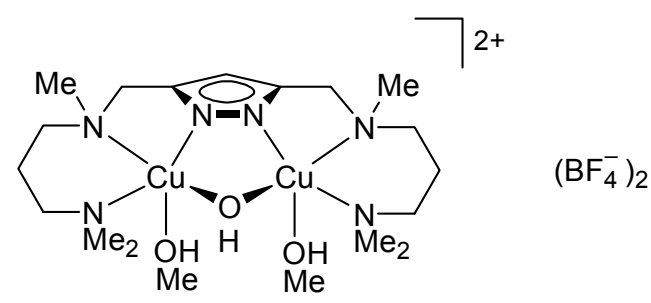

A solution of $\mathbf{H L}^{1}(0.08 \mathrm{~g}, 0.25 \mathrm{mmol})$ in methanol $(20 \mathrm{~mL})$ was treated with $\mathrm{KO} t \mathrm{Bu}(0.06 \mathrm{~g}$, $0.50 \mathrm{mmol})$ and stirred for $10 \mathrm{~min}$ at room temperature. $\mathrm{Cu}\left(\mathrm{BF}_{4}\right)_{2} \cdot 4 \mathrm{H}_{2} \mathrm{O}(0.15 \mathrm{~g}, 0.50 \mathrm{mmol})$ was then added and the green solution was stirred for $2 \mathrm{~h}$. After evaporation of the solvent, the crude product was redissolved in $5 \mathrm{ml}$ of $\mathrm{MeOH}$ and slow diffusion of $\mathrm{Et}_{2} \mathrm{O}$ into this solution led to the formation of blue-green crystals of $\mathbf{1}$.

Yield:

Empirical formula:

Molecular weight:

$\operatorname{IR}(\mathrm{KBr}) v\left(\mathrm{~cm}^{-1}\right)$ :

MS (FAB, glycerin) $m / z(\%)$ :

Elemental analysis $(\%)$ :

$$
0.12 \mathrm{~g}, 68 \%
$$

$\mathrm{C}_{19} \mathrm{H}_{44} \mathrm{Cu}_{2} \mathrm{~N}_{6} \mathrm{O}_{3} \mathrm{~B}_{2} \mathrm{~F}_{8}$

$705.3 \mathrm{~g} / \mathrm{mol}$

3494 (w), 1632 (m), 1466 (m), 1320 (w), 1082 (vs), 824 (w), 522

(w)

$449(100)\left[\mathrm{Cu}_{2} \mathrm{~L}^{1}\right]^{+}, 468(50)\left[\mathrm{Cu}_{2} \mathrm{~L}^{1}(\mathrm{OH})+2 \mathrm{H}\right]^{+}$

for $\mathrm{C}_{18} \mathrm{H}_{40} \mathrm{Cu}_{2} \mathrm{~N}_{6} \mathrm{O}_{2} \mathrm{~B}_{2} \mathrm{~F}_{8}$

calcd.: C 32.11, H 5.99, N 12.48

found: C 29.67, H 5.58, N 11.86

$\mathrm{UV} / \mathrm{vis}(\mathrm{MeCN}) \lambda(\mathrm{nm})[\varepsilon](\mathrm{L}$

$\left.\mathrm{mol}^{-1} \mathrm{~cm}^{-1}\right)$ : $\quad 628(282)$ 


\section{Preparation of $\left[\mathrm{Cu}_{2}\left(\mathrm{~L}^{2} \mathrm{O}\right)_{2}\right]\left(\mathrm{ClO}_{4}\right)_{2}(3)$}



A solution of $\mathbf{H L}^{2}(0.12 \mathrm{~g}, 0.25 \mathrm{mmol})$ in methanol $(20 \mathrm{~mL})$ was treated with $\mathrm{KO} t \mathrm{Bu}(0.06 \mathrm{~g}$, $0.50 \mathrm{mmol})$ and stirred for $10 \mathrm{~min}$ at room temperature. $\mathrm{Cu}\left(\mathrm{ClO}_{4}\right)_{2} \cdot 6 \mathrm{H}_{2} \mathrm{O}(0.18 \mathrm{~g}, 0.50 \mathrm{mmol})$ was then added and the green mixture was stirred for $2 \mathrm{~h}$. After evaporation of the solvent in vacuo, the crude product was redissolved in $10 \mathrm{ml}$ of EtOH and slow diffusion of petroleum ether (PE 40-60) into this solution led to the formation of blue-green crystals of $3 \cdot 0.47 \mathrm{H}_{2} \mathrm{O}$.

Yield:

Empirical formula:

Molecular weight:

$\operatorname{IR}(\mathrm{KBr}) v\left(\mathrm{~cm}^{-1}\right)$ :

MS (ESI, MeOH) $m / z(\%)$ :
$5 \mathrm{mg}, 3.0 \%$

$\mathrm{C}_{50} \mathrm{H}_{106} \mathrm{Cu}_{2} \mathrm{~N}_{16} \mathrm{O}_{10} \mathrm{Cl}_{2} \cdot 0.47 \mathrm{H}_{2} \mathrm{O}$

$1297.9 \mathrm{~g} / \mathrm{mol}$

3429 (m), 2958 (w), 1635 (w), 1467 (m), 1257 (w), 1152 (w),

1090 (vs), 801 (m), 625 (m), 503 (w)

1189 (95) $\left[\mathrm{Cu}_{2} \mathrm{~L}_{2}^{2} \mathrm{O}_{2}\left(\mathrm{ClO}_{4}\right)-2 \mathrm{H}\right]^{+}, 1125$ (100)

$\left[\mathrm{Cu}_{2} \mathrm{~L}_{2} \mathrm{O}_{2}(\mathrm{MeOH})\right]^{+}$ 


\section{Preparation of $\left[\mathrm{Cu}_{2} \mathrm{H}\left(\mathrm{L}^{2} \mathrm{O}\right)_{2}\right]\left(\mathrm{ClO}_{4}\right)_{3}(3 a)$}

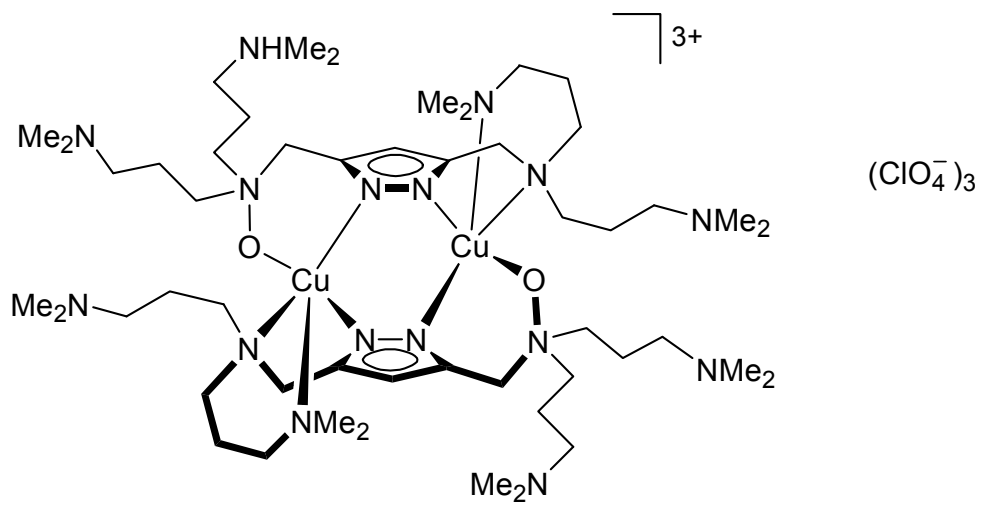

A solution of $\mathbf{H L}^{2}(0.12 \mathrm{~g}, 0.25 \mathrm{mmol})$ in methanol $(20 \mathrm{~mL})$ was treated with $\mathrm{KO} t \mathrm{Bu}(0.06 \mathrm{~g}$, $0.50 \mathrm{mmol})$ and stirred for $10 \mathrm{~min}$ at room temperature. $\mathrm{Cu}\left(\mathrm{ClO}_{4}\right)_{2} \cdot 6 \mathrm{H}_{2} \mathrm{O}(0.18 \mathrm{~g}, 0.50 \mathrm{mmol})$ was then added and the green solution was stirred for $2 \mathrm{~h}$. After evaporation of solvent, the crude product was redissolved in $10 \mathrm{ml}$ of $\mathrm{MeOH}$ and slow diffusion of $\mathrm{Et}_{2} \mathrm{O}$ into this solution led to the formation of blue-green crystals of $\mathbf{3 a} \cdot 3 \mathrm{MeOH}$.

Yield:

Empirical formula:

Molecular weight:

$\operatorname{IR}(\mathrm{KBr}) v\left(\mathrm{~cm}^{-1}\right)$ :

MS (ESI, $\mathrm{MeOH}) m / z(\%)$ :
$4 \mathrm{mg}, 2.2 \%$

$\mathrm{C}_{50} \mathrm{H}_{107} \mathrm{Cu}_{2} \mathrm{~N}_{16} \mathrm{O}_{15} \mathrm{Cl}_{3} \cdot 3 \mathrm{MeOH}$ $1486.0 \mathrm{~g} / \mathrm{mol}$ 3410 (w), 2963 (w), 1259 (s), 1094 (vs), 1025 (s), 802 (s), 625 (w), $503(\mathrm{w})$ $1389(15)\left[\mathrm{Cu}_{2} \mathrm{~L}_{2}^{2} \mathrm{O}_{2}\left(\mathrm{ClO}_{4}\right)_{3}-2 \mathrm{H}\right]^{+}, 1289(55)\left[\mathrm{Cu}_{2} \mathrm{~L}_{2}{ }_{2} \mathrm{O}_{2}\left(\mathrm{ClO}_{4}\right)_{2}-\right.$ $2 \mathrm{H}]^{+}, 1189(100)\left[\mathrm{Cu}_{2} \mathrm{~L}_{2} \mathrm{O}_{2}\left(\mathrm{ClO}_{4}\right)-2 \mathrm{H}\right]^{+}$ 


\section{Preparation of $\left[\mathrm{Cu}_{2}\left(\mathrm{~L}^{2} \mathrm{O}\right)_{2}\right]\left(\mathrm{BF}_{4}\right)_{2}(3 \mathrm{~b})$}

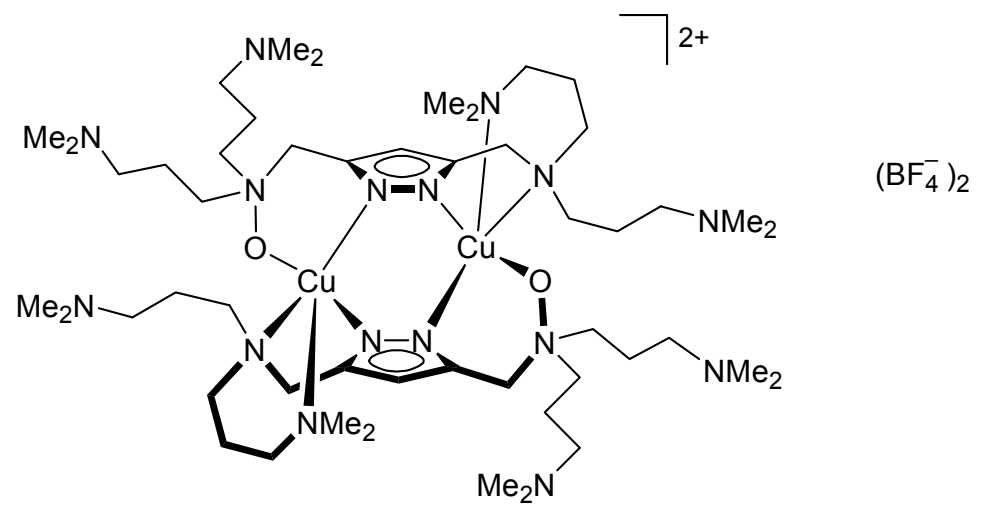

A solution of $\mathbf{H L}^{2}(0.12 \mathrm{~g}, 0.25 \mathrm{mmol})$ in methanol $(20 \mathrm{~mL})$ was treated with $\mathrm{KO} t \mathrm{Bu}(0.06 \mathrm{~g}$, $0.50 \mathrm{mmol})$ and stirred for $10 \mathrm{~min}$ at room temperature. $\mathrm{Cu}\left(\mathrm{BF}_{4}\right)_{2} \cdot 4 \mathrm{H}_{2} \mathrm{O}(0.13 \mathrm{~g}, 0.50 \mathrm{mmol})$ was then added and the green solution was stirred for $2 \mathrm{~h}$. After evaporation of the solvent in vacuo, the crude product was dissolved in $10 \mathrm{ml}$ of $\mathrm{CH}_{2} \mathrm{Cl}_{2}$ and slow diffusion of $\mathrm{Et}_{2} \mathrm{O}$ into this solution led to the formation of blue-green crystals of $\mathbf{3 b}$.

Yield:

Empirical formula:

Molecular weight:

$\operatorname{IR}(\mathrm{KBr}) v\left(\mathrm{~cm}^{-1}\right)$ :

MS (ESI, MeCN) $m / z(\%)$ :
$3.5 \mathrm{mg}, 1.9 \%$

$\mathrm{C}_{50} \mathrm{H}_{106} \mathrm{Cu}_{2} \mathrm{~N}_{16} \mathrm{O}_{2} \mathrm{~B}_{2} \mathrm{~F}_{8}$

$1264.2 \mathrm{~g} / \mathrm{mol}$

3434 (m), 2961 (w), 2820 (w), 2767 (w), 1634 (w), 1464 (m),

1257 (m), $1156(\mathrm{w}), 1082$ (vs), 801 (s)

$1177(5)\left[\mathrm{Cu}_{2} \mathrm{~L}_{2}^{2} \mathrm{O}_{2}\left(\mathrm{BF}_{4}\right)^{+}\right]$ 


\section{Preparation of $\left[\mathrm{Cu}_{2} \mathrm{~L}^{3}(\mu-\mathrm{OH})\right]\left(\mathrm{ClO}_{4}\right)_{2}(4)$}

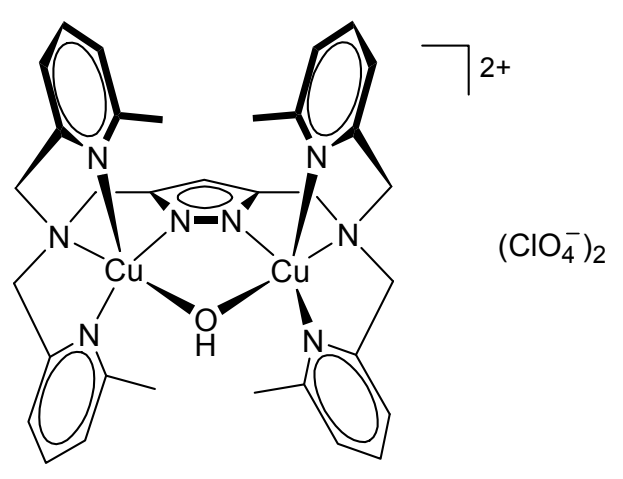

A solution of $\mathbf{H L}^{3}(0.14 \mathrm{~g}, 0.25 \mathrm{mmol})$ in $\mathrm{MeCN} / \mathrm{MeOH}(20 \mathrm{~mL} ; 1: 1 \mathrm{v} / \mathrm{v})$ was treated with $\mathrm{KO} t \mathrm{Bu}(0.06 \mathrm{~g}, 0.50 \mathrm{mmol})$ and stirred for $10 \mathrm{~min}$ at room temperature. $\mathrm{Cu}\left(\mathrm{ClO}_{4}\right)_{2} \cdot 6 \mathrm{H}_{2} \mathrm{O}$ $(0.18 \mathrm{~g}, 0.50 \mathrm{mmol})$ was then added and the green solution was stirred for $2 \mathrm{~h}$. After evaporation of the solvent, the crude product was redissolved in $5 \mathrm{ml}$ of $\mathrm{MeCN} / \mathrm{CH}_{2} \mathrm{Cl}_{2}$ and slow diffusion of $\mathrm{Et}_{2} \mathrm{O}$ into this solution led to the formation of green crystals of 4 (insufficient quality for a high-resolution X-ray crystallographic analysis).

Yield:

Empirical formula:

Molecular weight:

$\operatorname{IR}(\mathrm{KBr}) v\left(\mathrm{~cm}^{-1}\right)$ :

MS (ESI, MeCN) $m / z(\%)$ :

Elemental analysis (\%):
$0.28 \mathrm{~g}, 63 \%$

$$
\mathrm{C}_{33} \mathrm{H}_{37} \mathrm{Cu}_{2} \mathrm{~N}_{8} \mathrm{O}_{9} \mathrm{Cl}_{2}
$$

$887.7 \mathrm{~g} / \mathrm{mol}$ 1577 (s), 1440 (m), 1369 (w), 1261 (w), 1090 (vs), 1009 (w), $959(\mathrm{w}), 882(\mathrm{w}), 786(\mathrm{~m}), 623(\mathrm{~s})$

$829(20)\left[\mathrm{Cu}_{2} \mathrm{~L}^{3}(\mathrm{OH})(\mathrm{MeCN}) \mathrm{ClO}_{4}\right]^{+}$

for $\mathrm{C}_{33} \mathrm{H}_{37} \mathrm{Cu}_{2} \mathrm{~N}_{8} \mathrm{O}_{9} \mathrm{Cl}_{2}$

calcd.: C 44.65, H 4.20, N 12.62

found: C 44.35, H 4.25, N 11.79

$\mathrm{UV} / \mathrm{vis}(\mathrm{MeCN}) \lambda(\mathrm{nm})[\varepsilon](\mathrm{L}$ $\left.\mathrm{mol}^{-1} \mathrm{~cm}^{-1}\right)$ : 


\section{Preparation of $\left[\mathrm{Cu}_{2} \mathrm{~L}^{4}(\mathrm{OMe})(\mathrm{MeOH})\right]\left(\mathrm{ClO}_{4}\right)_{2}(5 \mathrm{a})$}

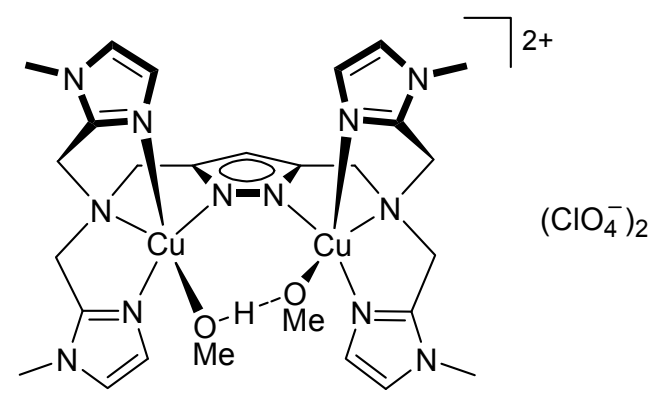

A solution of $\mathbf{H L}^{4}(2.63 \mathrm{~g}, 5.24 \mathrm{mmol})$ in methanol $(100 \mathrm{~mL})$ was treated with $\mathrm{KO} t \mathrm{Bu}(0.58$ $\mathrm{g}, 5.24 \mathrm{mmol})$ and stirred for $10 \mathrm{~min}$ at room temperature. $\mathrm{Cu}\left(\mathrm{NO}_{3}\right)_{2} \cdot 3 \mathrm{H}_{2} \mathrm{O}(2.51 \mathrm{~g}, 10.48$ mmol) was then added and stirring of the green solution was continued for $2 \mathrm{~h}$. After addition of $\mathrm{H}_{2} \mathrm{O}(900 \mathrm{~mL})$, the mixture was purified by ion-exchange chromatography on a SPSephadex C25 column. The main blue fraction was collected by washing the column with a $0.4 \mathrm{M}$ aqueous solution of $\mathrm{NaNO}_{3}$. The solvent was evaporated on a rotary evaporator and the residue extracted three times with $\mathrm{MeCN}$ to remove $\mathrm{NaNO}_{3}$ by filtration. The combined $\mathrm{MeCN}$ phases were evaporated to dryness and the residue re-dissolved in water. A $0.2 \mathrm{M}$ $\mathrm{NaOH}$ solution was then slowly added until the solution reached $\mathrm{pH} 9$, concomitant with a colour change from blue to deep green. Addition of excess $\mathrm{NaClO}_{4}$ yielded a green precipitate, which was separated by filtration and dried. Slow diffusion of $\mathrm{Et}_{2} \mathrm{O}$ into a solution of the crude product in $\mathrm{MeCN} / \mathrm{MeOH}(2: 1)$ led to the formation of green crystals of $\mathbf{5 a} \cdot \mathrm{MeCN}$.

Yield:

Empirical formula:

Molecular weight:

$\operatorname{IR}(\mathrm{KBr}) v\left(\mathrm{~cm}^{-1}\right)$ :

MS (FAB, glycerin) $m / z(\%)$ :

Elemental analysis (\%):
$2.92 \mathrm{~g}, 60 \%$

$\mathrm{C}_{27} \mathrm{H}_{40} \mathrm{Cu}_{2} \mathrm{~N}_{12} \mathrm{O}_{10} \mathrm{Cl}_{2} \cdot \mathrm{MeCN}$

$931.7 \mathrm{~g} / \mathrm{mol}$

3458 (s), 3132 (w), 1555 (m), 1509 (w), 1454 (w), $1381(\mathrm{w})$, $1286(\mathrm{w}), 1086$ (vs), 953 (w), 871 (w), 665 (w), 626 (m)

$726(10)\left[\mathrm{Cu}_{2} \mathrm{~L}^{4}\left(\mathrm{ClO}_{4}\right)\right]^{+}, 627$ (100) $\left[\mathrm{Cu}_{2} \mathrm{~L}^{4}\right]^{+}$

for $\mathrm{C}_{27} \mathrm{H}_{40} \mathrm{Cu}_{2} \mathrm{~N}_{12} \mathrm{O}_{10} \mathrm{Cl}_{2} \cdot \mathrm{MeCN}$

calcd.: C 34.80, H 4.29, N 18.04

found: C 34.82, H 4.27, N 18.96 
$\mathrm{UV} / \mathrm{vis}(\mathrm{MeCN} / \mathrm{MeOH}) \lambda(\mathrm{nm})$

$[\varepsilon]\left(\mathrm{L} \mathrm{mol}^{-1} \mathrm{~cm}^{-1}\right)$ :

Diffuse reflectance:

Preparation of $\left[\mathrm{Cu}_{2} \mathrm{~L}^{4}(\mathrm{OMe})(\mathrm{MeOH})\right]\left(\mathrm{NO}_{3}\right)_{2}(5 \mathrm{~b})$



A solution of $\mathbf{H L}^{4}(0.10 \mathrm{~g}, 0.20 \mathrm{mmol})$ in methanol $(50 \mathrm{~mL})$ was treated with two equivalents of $\mathrm{KO} t \mathrm{Bu}(0.04 \mathrm{~g}, 0.40 \mathrm{mmol})$ and the solution was stirred for $10 \mathrm{~min} . \mathrm{Cu}\left(\mathrm{NO}_{3}\right)_{2} \cdot 3 \mathrm{H}_{2} \mathrm{O}(0.09$ $\mathrm{g}, 0.40 \mathrm{mmol}$ ) was then added and stirring of the resulting green solution was continued for 2 $\mathrm{h}$ at room temperature. After evaporation of all volatile material, the remaining green solid was dissolved in a mixture of $\mathrm{MeCN} / \mathrm{MeOH}(2: 1)$. Slow diffusion of $\mathrm{Et}_{2} \mathrm{O}$ into this solution led to the formation of green crystals of $\mathbf{5 b} \cdot 2 \mathrm{MeOH}$.

Yield:

Empirical formula:

Molecular weight:

$\operatorname{IR}(\mathrm{KBr}) v\left(\mathrm{~cm}^{-1}\right)$ :

MS (FAB, glycerine) $m / z(\%)$ :

Elemental analysis (\%):

$$
0.10 \mathrm{~g}, 57 \%
$$

$$
\mathrm{C}_{27} \mathrm{H}_{40} \mathrm{Cu}_{2} \mathrm{~N}_{14} \mathrm{O}_{8} \cdot 2 \mathrm{MeOH}
$$

$879.9 \mathrm{~g} / \mathrm{mol}$

3429 (s), 2943 (w), 1508 (m), 1355 (vs), 1283 (w), 1160 (w), $1095(\mathrm{w}), 1036(\mathrm{w}), 951(\mathrm{w}), 874(\mathrm{w}), 759(\mathrm{w})$

689 (10) $\left[\mathrm{Cu}_{2} \mathrm{~L}^{4}\left(\mathrm{NO}_{3}\right)\right]^{+}, 627$ (100) $\left[\mathrm{Cu}_{2} \mathrm{~L}^{4}\right]^{+}$

for $\mathrm{C}_{27} \mathrm{H}_{40} \mathrm{Cu}_{2} \mathrm{~N}_{14} \mathrm{O}_{8} \cdot 2 \mathrm{MeOH}$

calcd.: C 39.59, H 5.50, N 22.29

found: C 39.76, H 5.35, N 22.73 
$\mathrm{UV} / \mathrm{vis}(\mathrm{MeCN} / \mathrm{MeOH}) \lambda(\mathrm{nm})$

$[\varepsilon]\left(\mathrm{L} \mathrm{mol}^{-1} \mathrm{~cm}^{-1}\right)$ :

726 sh (124), 962 (278)

\section{Preparation of $\left[\left\{\mathrm{Cu}_{2} \mathrm{~L}^{4}\left(\mathrm{NO}_{3}\right)\left(\mathrm{H}_{2} \mathrm{O}\right)\right\}_{2}\right]\left(\mathrm{NO}_{3}\right)_{4}(6)$}

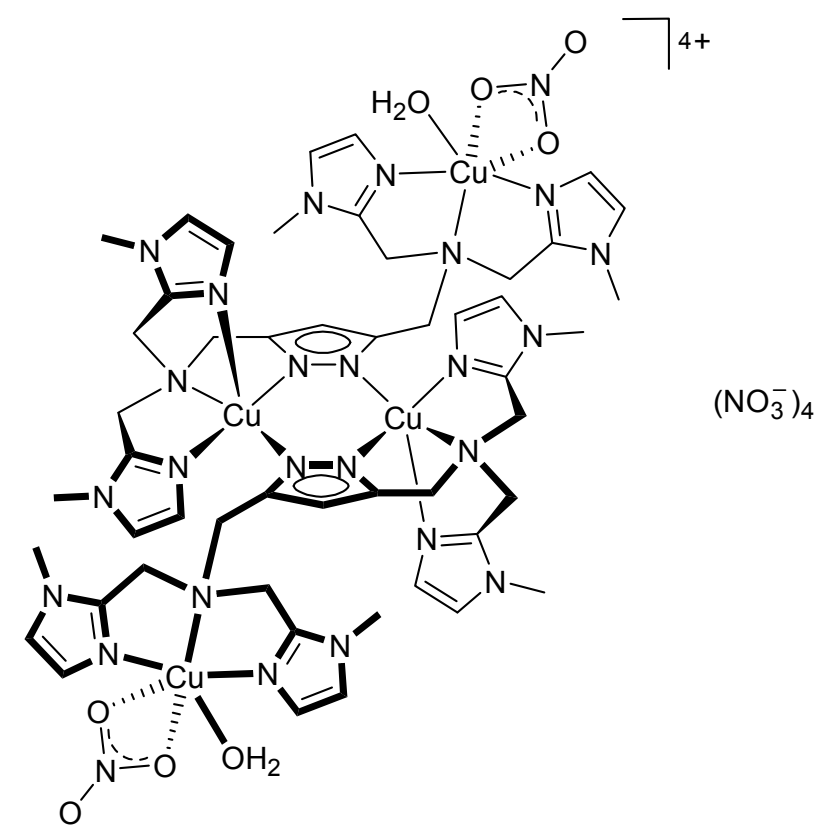

A solution of $\mathbf{H L}^{4}(2.63 \mathrm{~g}, 5.24 \mathrm{mmol})$ in methanol $(100 \mathrm{~mL})$ was treated with $\mathrm{KO} t \mathrm{Bu}(0.58$ g, $5.24 \mathrm{mmol})$ and stirred for $10 \mathrm{~min}$ at room temperature. $\mathrm{Cu}\left(\mathrm{NO}_{3}\right)_{2} \cdot 3 \mathrm{H}_{2} \mathrm{O}(2.51 \mathrm{~g}, 10.48$ mmol) was then added and stirring of the green solution was continued for $2 \mathrm{~h}$. After addition of $\mathrm{H}_{2} \mathrm{O}(900 \mathrm{~mL})$, the mixture was purified by ion-exchange chromatography on a SPSephadex C25 column. The main blue fraction was collected by washing the column with an aqueous $0.4 \mathrm{M} \mathrm{NaNO}_{3}$ solution. The solvent was evaporated and the residue extracted three times with $\mathrm{MeCN}$ to remove $\mathrm{NaNO}_{3}$ by filtration. The crude product was then redissolved in water and from this aqueous solution green-blue crystals of $6 \cdot 5 \mathrm{H}_{2} \mathrm{O}$ were obtained.

Yield:

Empirical formula:

Molecular weight:
$3.44 \mathrm{~g}, 75 \%$

$$
\mathrm{C}_{50} \mathrm{H}_{80} \mathrm{Cu}_{4} \mathrm{~N}_{30} \mathrm{O}_{25} \cdot 5 \mathrm{H}_{2} \mathrm{O}
$$

$1755.6 \mathrm{~g} / \mathrm{mol}$ 
$\operatorname{IR}(\mathrm{KBr}) v\left(\mathrm{~cm}^{-1}\right)$ :

MS (FAB, 3-NBA) $m / z(\%)$ :

Elemental analysis (\%):

\section{Preparation of $\left[\mathrm{Cu}_{2} \mathrm{HL}^{4}\left(\mathrm{H}_{2} \mathrm{O}\right)_{2}\left(\mathrm{ClO}_{4}\right)\right]\left(\mathrm{ClO}_{4}\right)_{3}(7)$}

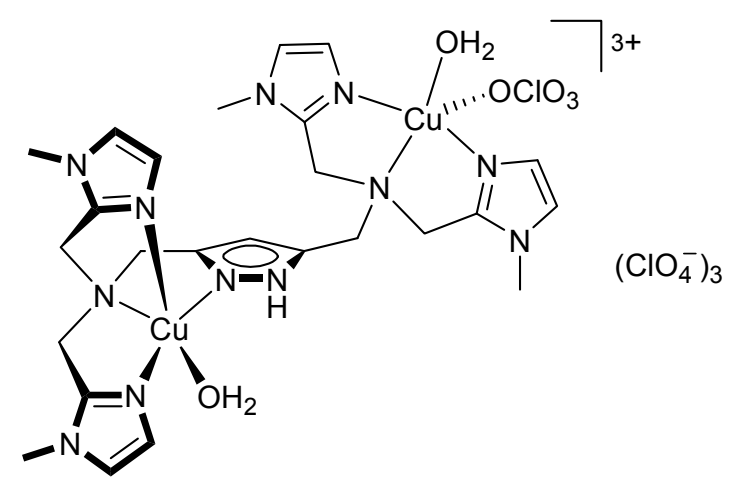

Aqueous $\mathrm{HClO}_{4}(5-10 \%(\mathrm{w}))$ was slowly added to a solution of $6(0.17 \mathrm{~g}, 0.10 \mathrm{mmol})$ in water $(10 \mathrm{~mL})$ until the $\mathrm{pH} 4$ was reached. After addition of methanol $(5 \mathrm{~mL})$ the solution was left standing for 2 days, during which time slow evaporation of the solvent led to the formation of blue crystals of $7 \cdot 2 \mathrm{H}_{2} \mathrm{O}$.

Yield:

$0.18 \mathrm{~g}, 86 \%$

Empirical formula:

$\mathrm{C}_{25} \mathrm{H}_{38} \mathrm{Cu}_{2} \mathrm{~N}_{12} \mathrm{O}_{18} \mathrm{Cl}_{4} \cdot 2 \mathrm{H}_{2} \mathrm{O}$

Molecular weight: $1099.6 \mathrm{~g} / \mathrm{mol}$

$\operatorname{IR}(\mathrm{KBr}) v\left(\mathrm{~cm}^{-1}\right)$ : (c) 3460 (w), $1628(w), 1555$ (w), 1509 (w), 1381 (w), 1088 (vs), 626 (m)

MS (FAB, 3-NBA) $m / z(\%): \quad 825(50)\left[\mathrm{Cu}_{2} \mathrm{~L}^{4}\left(\mathrm{ClO}_{4}\right)_{2}\right]^{+}, 726(55)\left[\mathrm{Cu}_{2} \mathrm{~L}^{4}\left(\mathrm{ClO}_{4}\right)\right]^{+}, 627(75)$ $\left[\mathrm{Cu}_{2} \mathrm{~L}^{4}\right]^{+}$ 
Elemental analysis (\%): $\quad$ for $\mathrm{C}_{25} \mathrm{H}_{38} \mathrm{Cu}_{2} \mathrm{~N}_{12} \mathrm{O}_{18} \mathrm{Cl}_{4} \cdot 2 \mathrm{H}_{2} \mathrm{O}$

calcd.: C 27.28, H 3.82, N 15.27

found: C 27.77, H 3.96, N 15.23

$\mathrm{UV} / \mathrm{vis}(\mathrm{MeCN} / \mathrm{MeOH}) \lambda$

(nm) $[\varepsilon]\left(\mathrm{L} \mathrm{mol}^{-1} \mathrm{~cm}^{-1}\right): \quad 677(96)$

Diffuse reflectance: $\quad 689$

\section{Preparation of $\left[\left\{\mathrm{Cu}_{2} \mathrm{~L}^{5}\right\}_{2}(\mu-\mathrm{OH})_{2}\right]\left(\mathrm{NO}_{3}\right)_{4}(8)$}

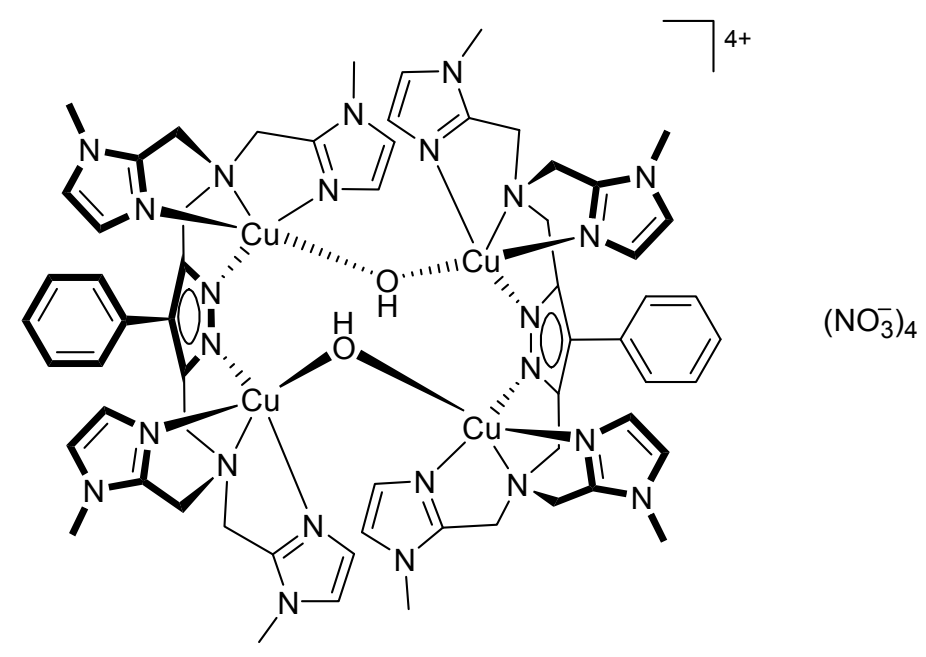

A solution of $\mathbf{H L}^{\mathbf{5}}(0.12 \mathrm{~g}, 0.20 \mathrm{mmol})$ in $\mathrm{MeCN} / \mathrm{MeOH}(10 \mathrm{~mL} ; 1: 1 \mathrm{v} / \mathrm{v})$ was treated with two equivalents of $\mathrm{KO} t \mathrm{Bu}(0.04 \mathrm{~g}, 0.40 \mathrm{mmol})$ and stirred for $10 \mathrm{~min} . \mathrm{Cu}\left(\mathrm{NO}_{3}\right)_{2} \cdot 3 \mathrm{H}_{2} \mathrm{O}(0.09$ $\mathrm{g}, 0.40 \mathrm{mmol}$ ) was then added and stirring of the resulting green solution was continued for 2 $\mathrm{h}$ at room temperature. After evaporation of all volatiles, the remaining green solid was dissolved in a mixture of $\mathrm{CH}_{3} \mathrm{CN} / \mathrm{CH}_{3} \mathrm{OH}(2: 1 \mathrm{v} / \mathrm{v})$. Slow diffusion of $\mathrm{Et}_{2} \mathrm{O}$ into this solution led to the formation of green crystals of $8 \cdot 0.5 \mathrm{MeCN}$.

Yield:

$0.1 \mathrm{~g}, 58 \%$

Empirical formula:

$\mathrm{C}_{62} \mathrm{H}_{76} \mathrm{Cu}_{4} \mathrm{~N}_{28} \mathrm{O}_{14} \cdot 0.5 \mathrm{MeCN}$

Molecular weight:

$1712.2 \mathrm{~g} / \mathrm{mol}$

$\operatorname{IR}(\mathrm{KBr}) v\left(\mathrm{~cm}^{-1}\right)$ : $3420(\mathrm{~m}), 1596(\mathrm{w}), 1509$ (w), $1383(\mathrm{vs}), 1163(\mathrm{w}), 1090(\mathrm{w})$, 
$1012(\mathrm{w}), 944(\mathrm{w}), 828(\mathrm{w}), 656(\mathrm{w})$

MS (FAB, glycerin) $m / z(\%): \quad 827(4)\left[\mathrm{Cu}_{2} \mathrm{~L}^{5}\left(\mathrm{NO}_{3}\right)_{2}\right]^{+}, 766(2.5)\left[\mathrm{Cu}_{2} \mathrm{~L}^{5}\left(\mathrm{NO}_{3}\right)\right]^{+}, 703(4.5)$ $\left[\mathrm{Cu}_{2} \mathrm{~L}^{5}\right]^{+}$

$\mathrm{UV} / \mathrm{vis}(\mathrm{MeCN} / \mathrm{MeOH}) \lambda(\mathrm{nm})$

$[\varepsilon]\left(\mathrm{L} \mathrm{mol}^{-1} \mathrm{~cm}^{-1}\right)$ : $\quad$ 726sh $(135), 995$ (280)

Preparation of $\left[\mathrm{L}^{4}\left\{\left(\mathrm{Me}_{3} \mathrm{CNC}\right)_{2} \mathrm{Cu}^{\mathrm{I}}\right\} \mathrm{Cu}^{\mathrm{II}}\right]_{2}\left(\mathrm{ClO}_{4}\right)_{4}(9)$

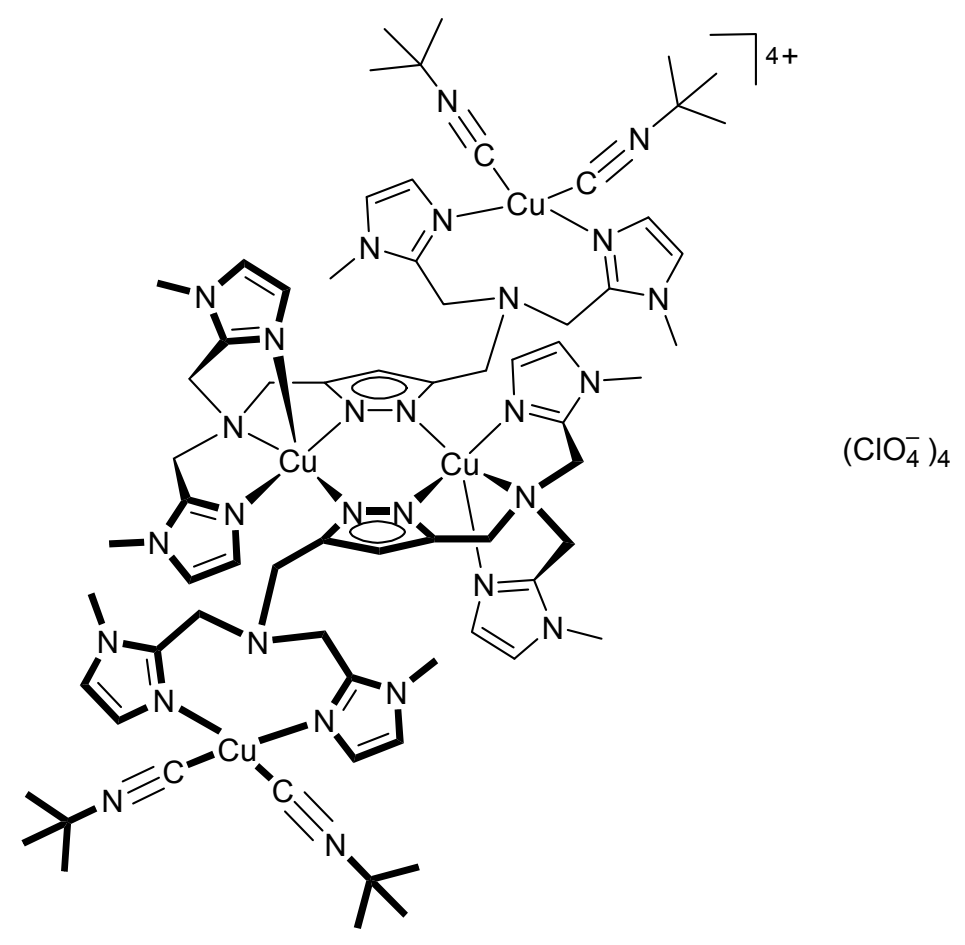

Under strict anaerobic conditions, complex 5a $(0.04 \mathrm{~g}, 0.05 \mathrm{mmol})$ was dissolved in $\mathrm{MeCN} / \mathrm{CH}_{2} \mathrm{Cl}_{2}$ (5 mL; ratio 2:3). The solution was stirred for $10 \mathrm{~min}$ and then TMP $(0.03 \mathrm{~g}$, $0.25 \mathrm{mmol}$ ) was added, which led to an immediate colour change of the reaction mixture from green to deep red. After $48 \mathrm{~h}$ of storage in an $\mathrm{N}_{2}$-filled glove box, the colour of the solution had changed to yellow-green and a green precipitate had formed. The solution was filtered and the green residue was dried under anaerobic conditions. The dry powder/solid was dissolved in $3 \mathrm{~mL}$ of $\mathrm{MeCN}$ and 2 equivalents $(0.1 \mathrm{mmol}, 0.012 \mathrm{~mL})$ of tert-butyl isocyanide per copper ion was added, anticipating the complete reduction of initial complex $\mathbf{5 a}$ to the 
desired $\mathrm{Cu}(\mathrm{I}) \mathrm{Cu}(\mathrm{II})$ complex. Slow diffusion of $\mathrm{Et}_{2} \mathrm{O}$ into the resulting intensely green coloured solution led to the formation of light-green crystals of $9 \cdot 7 \mathrm{MeCN}$.

Yield:

$0.045 \mathrm{~g}, 80 \%$

Empirical formula:

$\mathrm{C}_{70} \mathrm{H}_{102} \mathrm{Cu}_{4} \mathrm{~N}_{28} \mathrm{O}_{16} \mathrm{Cl}_{4} \cdot 7 \mathrm{MeCN}$

Molecular weight:

$2275.5 \mathrm{~g} / \mathrm{mol}$

$\operatorname{IR}(\mathrm{KBr}) v\left(\mathrm{~cm}^{-1}\right)$ : 3420 (s), 2173 (s), 2151 (s), 1628 (w), 1504 (m), 1449 (w), $1379(\mathrm{w}), 1285$ (w), $1090(\mathrm{vs}), 952(\mathrm{w}), 757$ (w), 625 (m)

$\mathrm{UV} / \mathrm{vis}(\mathrm{MeCN}) \lambda(\mathrm{nm})[\varepsilon](\mathrm{L}$ $\left.\mathrm{mol}^{-1} \mathrm{~cm}^{-1}\right)$ :

$695 \mathrm{sh}(119) 948(383)$

Preparation of $\left[\mathrm{L}^{5}\{(t \mathrm{BuNC}) \mathrm{Cu}\}_{3}\right]_{2}\left(\mathrm{PF}_{6}\right)_{4}(10)$

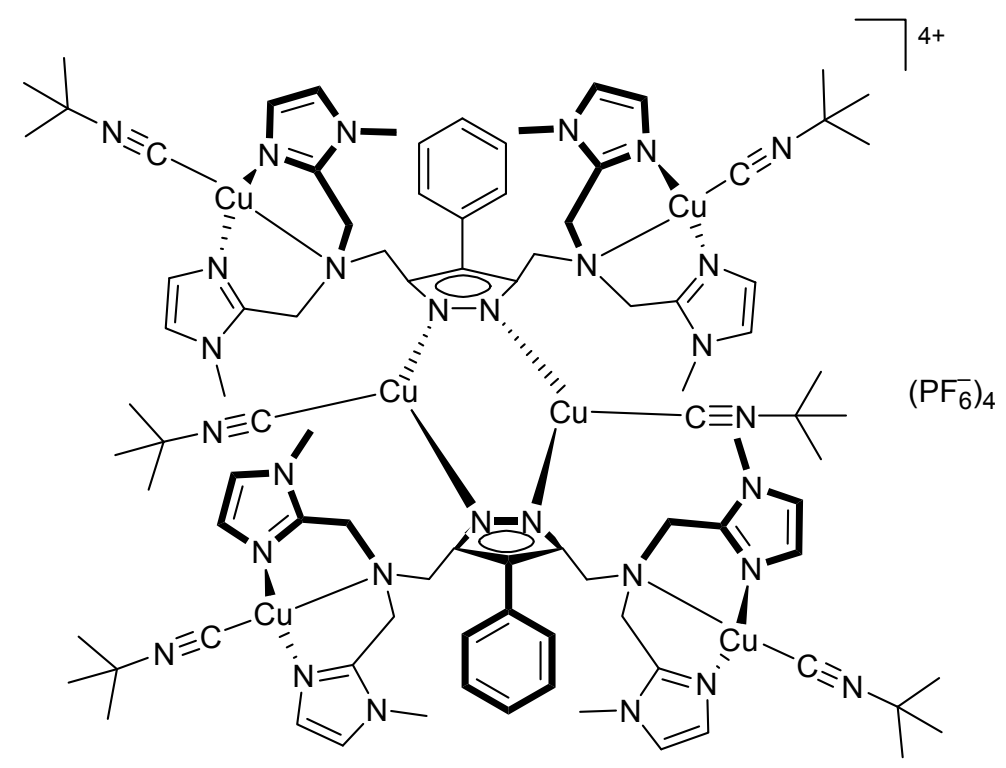

Under strict anaerobic conditions, a solution of $\mathbf{H L}^{\mathbf{5}}(0.12 \mathrm{~g}, 0.20 \mathrm{mmol})$ in $\mathrm{MeCN}(10 \mathrm{~mL})$ was treated with one equivalent of $\mathrm{KO} t \mathrm{Bu}(0.02 \mathrm{~g}, 0.20 \mathrm{mmol})$ and stirred for $2 \mathrm{~h}$. $\mathrm{Cu}(\mathrm{MeCN})_{4}\left(\mathrm{PF}_{6}\right)(0.15 \mathrm{~g}, 0.40 \mathrm{mmol})$ was then added and stirring of the resulting lightyellow solution was continued for $2 \mathrm{~h}$ at room temperature. Subsequently, one equivalent $(0.40 \mathrm{mmol}, 0.048 \mathrm{~mL})$ of tert-butyl isocyanide $(\mathrm{BuNC})(1: 1$ ratio to $\mathrm{Cu})$ was added and 
stirring was continued for $30 \mathrm{~min}$. Slow diffusion of $\mathrm{Et}_{2} \mathrm{O}$ into this solution led to the formation of colourless crystals of $\mathbf{1 0}$.

Yield:

Empirical formula:

Molecular weight:

$\operatorname{IR}(\mathrm{KBr}) v\left(\mathrm{~cm}^{-1}\right)$ :

${ }^{1} \mathrm{H}-\mathrm{NMR}\left(\mathrm{CD}_{3} \mathrm{CN}\right) \delta(\mathrm{ppm})$ :
$0.13 \mathrm{~g}, 68 \%$

$\mathrm{C}_{92} \mathrm{H}_{128} \mathrm{Cu}_{6} \mathrm{~N}_{30} \mathrm{P}_{4} \mathrm{~F}_{24}$

$2615.6 \mathrm{~g} / \mathrm{mol}$

2985 (w), 2164 (vs), 1541 (w), 1504 (m), 1448 (w), 1371 (m), $1284(\mathrm{~m}), 1202$ (m), 1084 (s), 842 (vs), 758 (m), 558 (m)

$1.44(\mathrm{~s}, t \mathrm{Bu}, 27 \mathrm{H}), 3.38\left(\mathrm{~s}, \mathrm{CH}_{3}, 12 \mathrm{H}\right), 3.55\left(\mathrm{~s}, \mathrm{NCH}_{2}, 8 \mathrm{H}\right)$,

$3.86\left(\mathrm{~s}, \mathrm{pzCH}_{2}, 4 \mathrm{H}\right), 6.94$ (m, im-CH, 8H), 7.32 (m, Ar-CH,

\section{Preparation of $\left[\mathrm{Cu}_{2} \mathrm{~L}^{4}\left(\mathrm{OC}_{6} \mathrm{~F}_{5}\right)(\mathrm{MeOH})\right]\left(\mathrm{ClO}_{4}\right)_{2}(11)$}

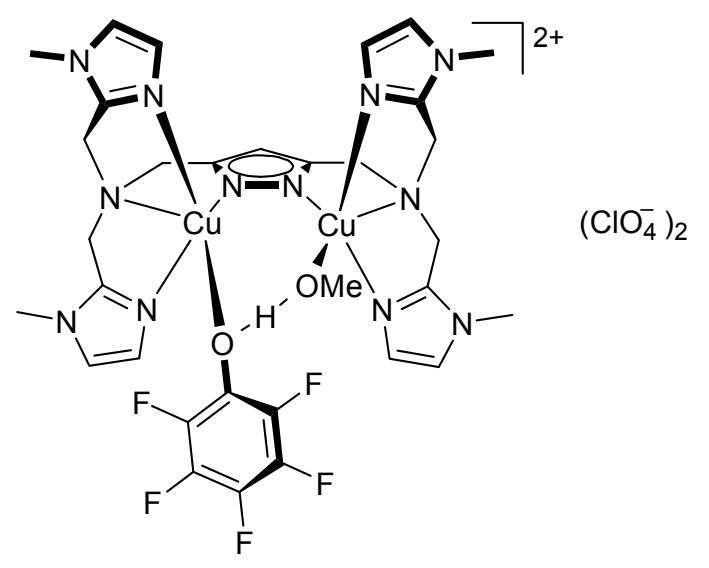

To a solution of $\mathbf{H L}^{4}(0.10 \mathrm{~g}, 0.20 \mathrm{mmol})$ in methanol $(50 \mathrm{~mL})$ was added $\mathrm{KO} t \mathrm{Bu}(0.04 \mathrm{~g}$, $0.40 \mathrm{mmol})$ and the solution was stirred for $10 \mathrm{~min} . \mathrm{Cu}\left(\mathrm{ClO}_{4}\right)_{2} \cdot 6 \mathrm{H}_{2} \mathrm{O}(0.15 \mathrm{~g}, 0.40 \mathrm{mmol})$ was then added and the green solution was stirred at room temperature for $2 \mathrm{~h}$; a small amount of green precipitate formed during this time. Subsequently, pentafluorophenol $\left(\mathrm{C}_{6} \mathrm{H}_{5} \mathrm{OH}\right)(0.36$ g, $2.00 \mathrm{mmol}$ ) was added to the green mixture, concomitant with an immediate colour-change of the reaction from green to yellow-green. After additional stirring for $2 \mathrm{~h}$, the solution was filtered and layered with $\mathrm{Et}_{2} \mathrm{O}$, yielding yellow-green crystals of $11 \cdot \mathrm{MeOH}$ overnight. 
Yield:

0.12 g, $56 \%$

Empirical formula:

$\mathrm{C}_{32} \mathrm{H}_{37} \mathrm{Cu}_{2} \mathrm{~N}_{12} \mathrm{O}_{10} \mathrm{~F}_{5} \mathrm{Cl}_{2} \cdot \mathrm{MeOH}$

Molecular weight:

$1074.7 \mathrm{~g} / \mathrm{mol}$

$\operatorname{IR}(\mathrm{KBr}) v\left(\mathrm{~cm}^{-1}\right)$ :

3401 (w), 1504 (vs), 1282 (w), 1101 (vs), 995 (m), 877 (w), 760

(m), $625(\mathrm{~m})$

MS (FAB, 3-NBA) $m / z(\%): 1009(15)\left[\mathrm{Cu}_{2} \mathrm{~L}^{4}\left(\mathrm{C}_{6} \mathrm{~F}_{5} \mathrm{O}\right)\left(\mathrm{ClO}_{4}\right)_{2}\right]^{+}, 910(15)$

$\left[\mathrm{Cu}_{2} \mathrm{~L}^{4}\left(\mathrm{C}_{6} \mathrm{~F}_{5} \mathrm{O}\right)\left(\mathrm{ClO}_{4}\right)\right]^{+}, 726(25)\left[\mathrm{Cu}_{2} \mathrm{~L}^{4}\left(\mathrm{ClO}_{4}\right)\right]^{+}, 627(100)$

$\left[\mathrm{Cu}_{2} \mathrm{~L}^{4}\right]^{+}$

$\mathrm{UV} / \mathrm{vis}(\mathrm{MeCN}) \lambda(\mathrm{nm})[\varepsilon](\mathrm{L}$

$\left.\mathrm{mol}^{-1} \mathrm{~cm}^{-1}\right)$ : $\quad 397(1240), 989(250)$

Preparation of $\left[\mathrm{CuL}^{4}\left(\mathrm{OC}_{6} \mathrm{H}_{4} \mathrm{C}(\mathrm{O}) \mathrm{NH}_{2}\right) \mathrm{Cu}(\mathrm{MeOH})\right]\left(\mathrm{NO}_{3}\right)_{2}$ (12)

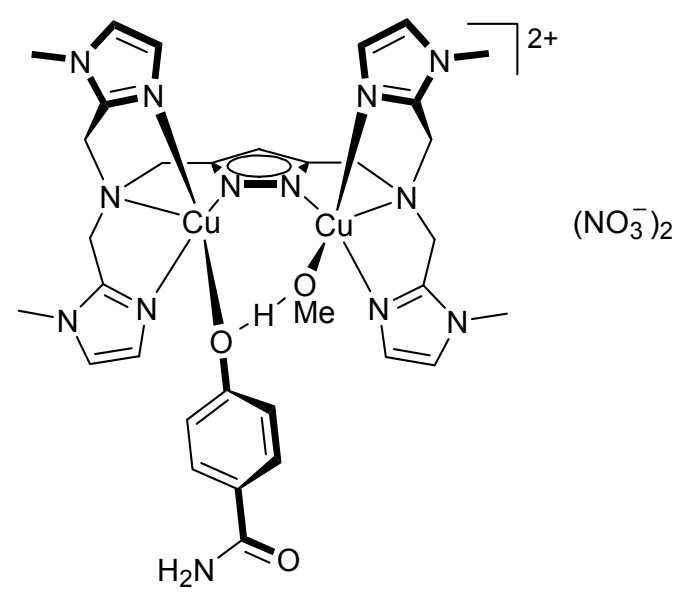

To $0.10 \mathrm{~g}(0.20 \mathrm{mmol})$ of $\mathbf{H L}^{4}$ in $50 \mathrm{~mL}$ methanol, $0.04 \mathrm{~g}(0.40 \mathrm{mmol})$ of potassium tertbutylate $(\mathrm{KO} t \mathrm{Bu})$ was added. The solution was stirred for $10 \mathrm{~min}$ and $0.09 \mathrm{~g}(0.40 \mathrm{mmol})$ of $\mathrm{Cu}\left(\mathrm{NO}_{3}\right)_{2} \cdot 3 \mathrm{H}_{2} \mathrm{O}$ was then added. The green solution was stirred at room temperature for $2 \mathrm{~h}$ and then $0.274 \mathrm{~g}(2 \mathrm{mmol})$ of 4-hydroxybenzamide was added as a powder to the green mixture. The colour of the reaction mixture changed from green to yellow-brown. After additional stirring for $2 \mathrm{~h}$, the solution was filtered and layered with $\mathrm{Et}_{2} \mathrm{O}$, yielding brown crystals of $\mathbf{1 2} \cdot 4 \mathrm{MeOH}$ overnight. 
Yield:

Empirical formula:

Molecular weight:

$\operatorname{IR}(\mathrm{KBr}) v\left(\mathrm{~cm}^{-1}\right)$ :

MS (ESI, MeCN) $m / z(\%)$ :

Elemental analysis (\%):
$0.18 \mathrm{~g}, 87 \%$

$$
\mathrm{C}_{33} \mathrm{H}_{43} \mathrm{Cu}_{2} \mathrm{~N}_{15} \mathrm{O}_{9} \cdot 4 \mathrm{MeOH}
$$

$1049.1 \mathrm{~g} / \mathrm{mol}$

3410 (w), 1653 (m), 1593 (m), 1505 (s), 1381 (vs), 1282 (s), 1163

(w), $1092(\mathrm{w}), 1031(\mathrm{w}), 1001(\mathrm{w}), 949(\mathrm{w}), 852(\mathrm{w}), 758(\mathrm{~m})$,

$666(\mathrm{w})$

$825(5)\left[\mathrm{Cu}_{2} \mathrm{~L}^{4}\left(\mathrm{OC}_{6} \mathrm{H}_{4} \mathrm{C}(\mathrm{O}) \mathrm{NH}_{2}\right) \mathrm{NO}_{3}\right]^{+}, 751(8)\left[\mathrm{Cu}_{2} \mathrm{~L}^{4}\left(\mathrm{NO}_{3}\right)_{2}\right]^{+}$, 689 (10) $\left[\mathrm{Cu}_{2} \mathrm{~L}^{4}\left(\mathrm{NO}_{3}\right)\right]^{+}, 627(9)\left[\mathrm{Cu}_{2} \mathrm{~L}^{4}\right]^{+}$

for $\mathrm{C}_{33} \mathrm{H}_{43} \mathrm{Cu}_{2} \mathrm{~N}_{15} \mathrm{O}_{9} \cdot 2 \mathrm{MeOH}$

calcd.: C 42.68, H 5.22, N 21.33

found: C 38.71, H 4.80, N 21.27

$\mathrm{UV} / \mathrm{vis}(\mathrm{MeCN}) \lambda(\mathrm{nm})[\varepsilon](\mathrm{L}$ $\left.\mathrm{mol}^{-1} \mathrm{~cm}^{-1}\right)$ :

446 (1210), 983 (226)

\section{Preparation of $\left[\left\{\mathrm{Cu}_{2} \mathrm{~L}^{4}\right\}(\mu-\mathrm{CN})_{2}\right]\left(\mathrm{ClO}_{4}\right)_{4}(13)$}

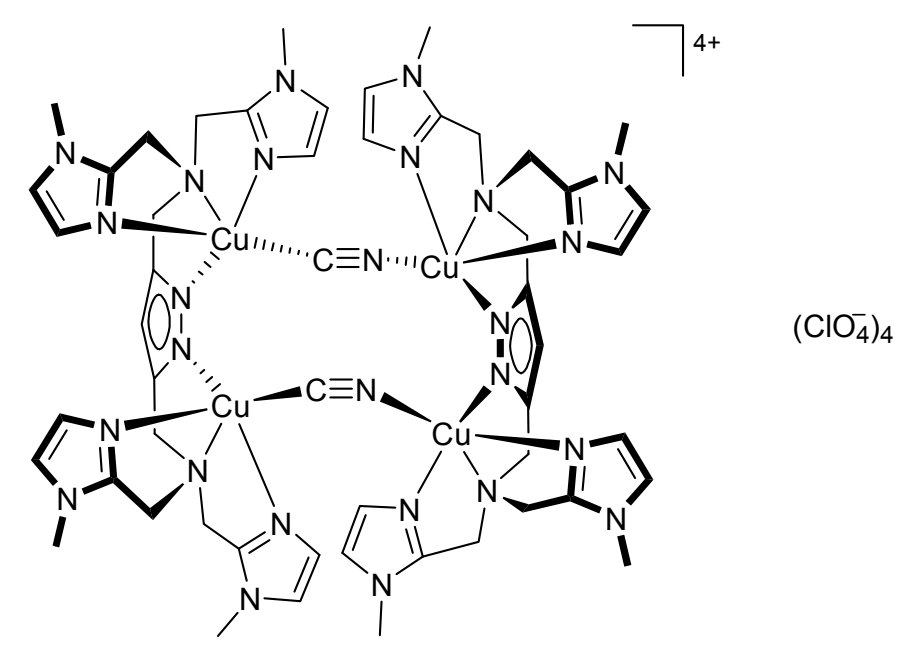

To a $47 \mathrm{mM}$ solution of complex 5a in $\mathrm{MeCN}\left(3 \mathrm{~mL}\right.$ ), a $47 \mathrm{mM}$ solution of $\mathrm{HOCF}_{3} \mathrm{BP}$ in $\mathrm{MeCN}$ was added in a stepwise manner with the aim to perform a Job experiment. Addition of the substrate led to the usual formation of a red coloured copper-phenolate species, but this proved to be only a short-lived complex, as further unexpected colour change to deep green 
occurred within $2 \mathrm{~h}$ after Job experiment was finished. Slow diffusion of $\mathrm{Et}_{2} \mathrm{O}$ into the green mixture of $\mathrm{HOCF}_{3} \mathrm{BP}$ and $\mathbf{5 a}$ induced the formation of dark green crystals of $\mathbf{1 3} \cdot 4 \mathrm{MeCN}$.

Yield:

Empirical formula:

Molecular weight:

$\operatorname{IR}(\mathrm{KBr}) v\left(\mathrm{~cm}^{-1}\right)$ :

MS (FAB, 3-NBA) $m / z(\%)$ :

$\mathrm{UV} / \mathrm{vis}(\mathrm{MeCN}) \lambda(\mathrm{nm})[\varepsilon](\mathrm{L}$ $\left.\mathrm{mol}^{-1} \mathrm{~cm}^{-1}\right)$ :

$$
0.14 \mathrm{~g}, 50 \%
$$

\section{$\mathrm{C}_{52} \mathrm{H}_{66} \mathrm{Cu}_{4} \mathrm{~N}_{26} \mathrm{O}_{16} \mathrm{Cl}_{4} \cdot 4 \mathrm{MeCN}$}

$1871.4 \mathrm{~g} / \mathrm{mol}$

3420 (s), $3123(\mathrm{w}), 2927(\mathrm{w}), 2174(\mathrm{w}), 2017(\mathrm{w}), 1628(\mathrm{w})$, $1553(\mathrm{~m}), 1509$ (s), 1451 (w), $1356(\mathrm{w}), 1284(\mathrm{w}), 1089$ (vs), $949(\mathrm{w}), 871(\mathrm{w}), 758(\mathrm{~m}), 625(\mathrm{~s}), 449(\mathrm{w})$

$1508(5)\left[\mathrm{Cu}_{4} \mathrm{~L}_{2}^{4}(\mathrm{CN})_{2}\left(\mathrm{ClO}_{4}\right)_{2}\right]^{+}, 754(15)\left[\mathrm{Cu}_{2} \mathrm{~L}^{4}(\mathrm{CN})\left(\mathrm{ClO}_{4}\right)\right]^{+}$, $728(25)\left[\mathrm{Cu}_{2} \mathrm{~L}^{4}\left(\mathrm{ClO}_{4}\right)\right]^{+}, 627(100)\left[\mathrm{Cu}_{2} \mathrm{~L}^{4}\right]^{+}$

$671(276), 879(306)$

\section{Preparation of $\left[\mathrm{Cu}_{5} \mathrm{~L}_{2}^{4}\left(\mathrm{C}_{6} \mathrm{Cl}_{4} \mathrm{O}_{2}\right)_{3}(\mathrm{DMF})_{2}\right]\left(\mathrm{ClO}_{4}\right)_{2}(14)$}

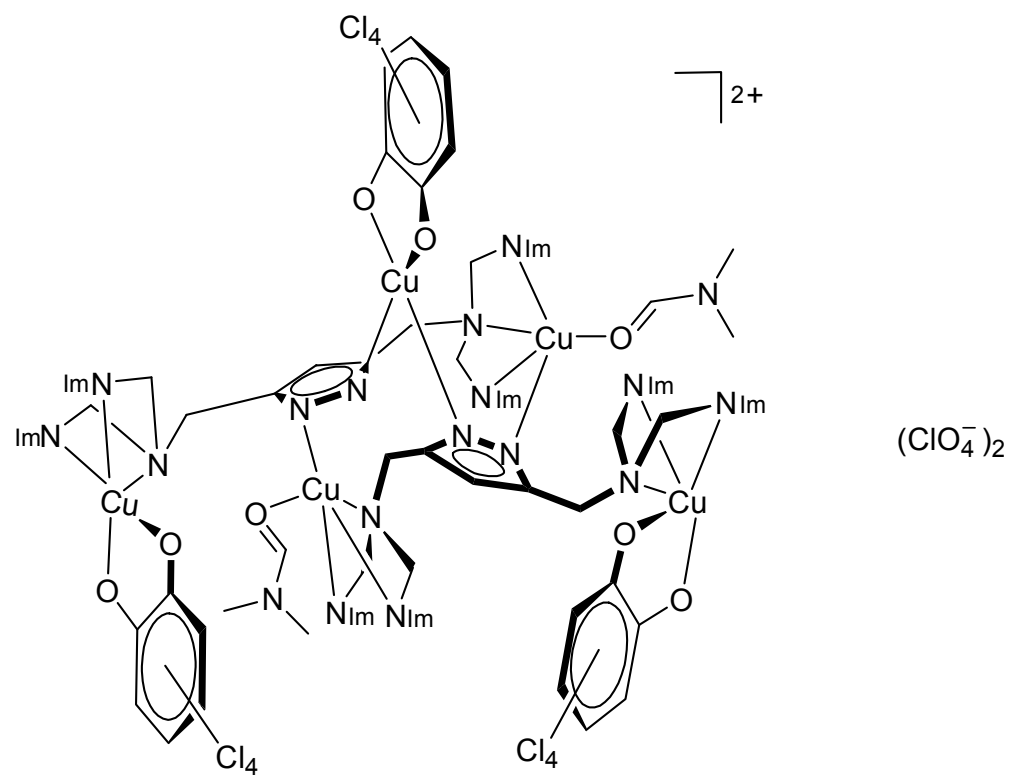

To a solution of $\mathbf{H L}^{4}(0.10 \mathrm{~g}, 0.20 \mathrm{mmol})$ in methanol $(50 \mathrm{~mL})$ were added two equivalents of $\mathrm{KO} t \mathrm{Bu}(0.04 \mathrm{~g}, 0.40 \mathrm{mmol})$ and subsequently, after $10 \mathrm{~min}$ of stirring, two equivalents of $\mathrm{Cu}\left(\mathrm{ClO}_{4}\right)_{2} \cdot 6 \mathrm{H}_{2} \mathrm{O}(0.14 \mathrm{~g}, 0.40 \mathrm{mmol})$. The reaction was completed by addition of one 
equivalent of tetrachlorocatechol (TCC) $(0.05 \mathrm{~g}, 0.20 \mathrm{mmol})$, which was pretreated with one equivalent of $\mathrm{KO} t \mathrm{Bu}(0.02 \mathrm{~g}, 0.20 \mathrm{mmol})$ to deprotonate one of the $\mathrm{OH}$-groups. The colour of the reaction mixture changed from green to dark-brown. After the solvent was evaporated, the crude residue was dissolved in a MeCN/DMF mixture from which single crystals of complex 14 were obtained by slow diffusion of $\mathrm{Et}_{2} \mathrm{O}$.

Yield:

Empirical formula:

Molecular weight:

$\operatorname{IR}(\mathrm{KBr}) \vee\left(\mathrm{cm}^{-1}\right)$ :

MS (ESI, MeCN) $m / z(\%)$ :

Elemental analysis (\%):

$$
0.18 \mathrm{~g}, 75 \%
$$

$\mathrm{C}_{74} \mathrm{H}_{80} \mathrm{Cu}_{5} \mathrm{~N}_{26} \mathrm{O}_{16} \mathrm{Cl}_{14}$

$2403.6 \mathrm{~g} / \mathrm{mol}$

$3411(\mathrm{~m}), 3129(\mathrm{w}), 1630(\mathrm{w}), 1542(\mathrm{w}), 1509$ (m), 1452 (s), 1372 (w), 1287 (w), 1255 (m), 1089 (vs), 966 (w), 886 (w), 796

$(\mathrm{w}), 749(\mathrm{~m}), 623(\mathrm{~s})$

1183 (10) $\left[\mathrm{Cu}_{3} \mathrm{~L}^{4}(\mathrm{TCC})_{2}\right]^{+}, 937(60)\left[\mathrm{Cu}_{2} \mathrm{~L}^{4}(\mathrm{TCC})(\mathrm{MeOH})_{2}\right]^{+}$, $872(75)\left[\mathrm{Cu}_{2} \mathrm{~L}^{4}(\mathrm{TCC})\right]^{+}, 627$ (100) $\left[\mathrm{Cu}_{2} \mathrm{~L}^{4}\right]^{+}$

for $\mathrm{C}_{74} \mathrm{H}_{80} \mathrm{Cu}_{5} \mathrm{~N}_{26} \mathrm{O}_{16} \mathrm{Cl}_{14} \cdot 6 \mathrm{H}_{2} \mathrm{O}$

calcd.: C 35.39, H 3.69, N 14.50

found: C 34.28, H 3.70, N 14.92

$\mathrm{UV} / \mathrm{vis}(\mathrm{MeCN}) \lambda(\mathrm{nm})[\varepsilon](\mathrm{L}$ $\left.\mathrm{mol}^{-1} \mathrm{~cm}^{-1}\right)$ : 


\section{Preparation of $\left[\mathrm{Cu}_{4} \mathrm{~L}_{2}{ }_{2}\left\{\mathrm{Cu}_{2}\left(\left(\mathrm{C}_{8} \mathrm{H}_{8} \mathrm{O}\right)_{3}\right)_{2}\right\}\right]\left(\mathrm{ClO}_{4}\right)_{4}(15)$}

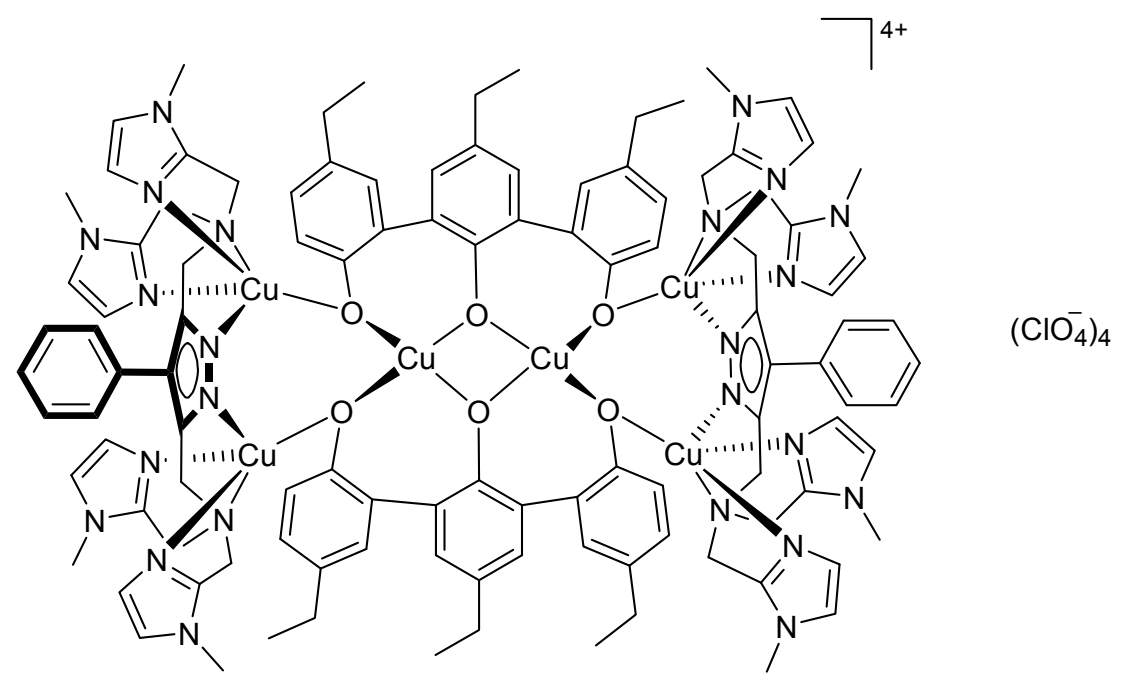

A solution of $\mathbf{H L}^{\mathbf{5}}(0.12 \mathrm{~g}, 0.20 \mathrm{mmol})$ in $10 \mathrm{~mL}$ methanol was treated with two equivalents of $\mathrm{KO} t \mathrm{Bu}(0.04 \mathrm{~g}, 0.40 \mathrm{mmol})$ and the solution stirred for $10 \mathrm{~min} . \mathrm{Cu}\left(\mathrm{ClO}_{4}\right)_{2} \cdot 6 \mathrm{H}_{2} \mathrm{O}(0.15 \mathrm{~g}$, $0.40 \mathrm{mmol}$ ) was then added and stirring of the resulting green mixture continued for $2 \mathrm{~h}$ at room temperature. After evaporation of all volatile material the remaining green solid was dissolved in $\mathrm{MeCN}$ and insoluble by-product $\left(\mathrm{KClO}_{4}\right)$ was filtered off. To the again redissolved pure powder of complex ten equivalents of $\mathrm{C}_{8} \mathrm{H}_{9} \mathrm{OH}$ were added with following addition of one equivalent of $\mathrm{KO} t \mathrm{Bu}$. The colour of reaction mixture changed to deep red. Slow diffusion of $\mathrm{Et}_{2} \mathrm{O}$ into this solution led to the formation of black crystals of $\mathbf{1 5}$.

Yield:

Empirical formula:

Molecular weight:

$\operatorname{IR}(\mathrm{KBr}) v\left(\mathrm{~cm}^{-1}\right)$ :

MS (ESI-HR, MeCN)

$m / z(\%)$ :
$0.011 \mathrm{~g}, 6.5 \%$

$\mathrm{C}_{110} \mathrm{H}_{122} \mathrm{Cu}_{6} \mathrm{~N}_{24} \mathrm{O}_{22} \mathrm{Cl}_{4}$

$2647.5 \mathrm{~g} / \mathrm{mol}$

$3414(w), 3229(w), 3126(w), 2942(w), 1625(m), 1555(m)$,

1506 (s), 1439 (m), 1375 (w), 1287 (w), 1112 (vs), 1088 (vs), 987

(w), $955(\mathrm{w}), 845(\mathrm{w}), 757(\mathrm{~m}), 625(\mathrm{~s})$

calcd.: 1224.22790

found: $1224.23\left[\mathrm{Cu}_{4} \mathrm{~L}_{2}^{5}\left\{\mathrm{Cu}_{2}\left(\left(\mathrm{C}_{8} \mathrm{H}_{8} \mathrm{O}\right)_{3}\right)_{2}\right\}\left(\mathrm{ClO}_{4}\right)_{2}\right]^{2+}$

$\mathrm{UV} / \mathrm{vis}(\mathrm{MeCN}) \lambda(\mathrm{nm})[\varepsilon](\mathrm{L}$

$\left.\mathrm{mol}^{-1} \mathrm{~cm}^{-1}\right)$ :

$732,972 *$

Diffuse reflectance:

427, 633, 1039

$* \varepsilon$ is not given: big errors in calculations because of the very diluted solution of $\mathbf{1 5}$ 


\section{Preparation of $\left[\mathrm{HL}^{4}\left\{\mathrm{CuBr}_{2}\right]\left(\mathrm{ClO}_{4}\right)_{2}\right](17)$}

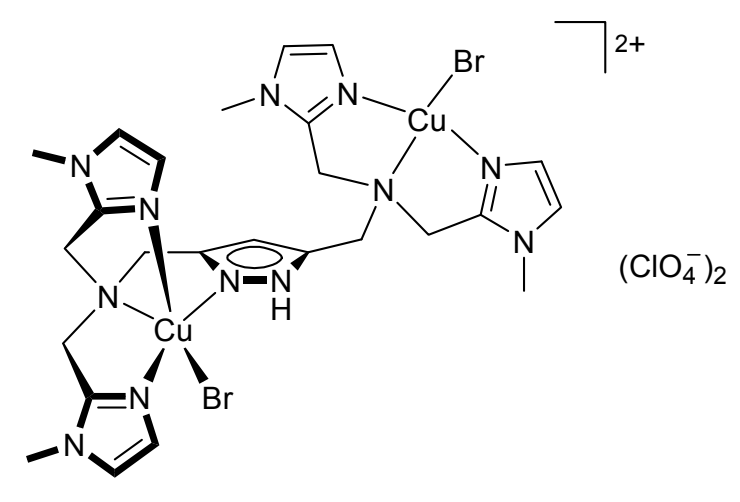

Complex 5a (0.05 g, $0.05 \mathrm{mmol})$ was dissolved in $\mathrm{MeCN}(10 \mathrm{~mL})$. The green solution was stirred for $10 \mathrm{~min}$ and then 4-bromo-2,6-dimethylphenol (DMBrP) (0.1 g, $0.5 \mathrm{mmol})$ was added. An immediate colour change of the reaction mixture from green to deep-red was observed concomitant with another colour change to deep green within 10 min. The solution was left standing for one day, during which time slow evaporation of the solvent led to the formation of blue crystals of $17 \cdot \mathrm{MeCN}$.

Yield:

Empirical formula:

Molecular weight:

$\operatorname{IR}(\mathrm{KBr}) v\left(\mathrm{~cm}^{-1}\right)$ :
$0.04 \mathrm{~g}, 71 \%$

$\mathrm{C}_{25} \mathrm{H}_{34} \mathrm{Cu}_{2} \mathrm{~N}_{12} \mathrm{O}_{8} \mathrm{Br}_{2} \mathrm{Cl}_{2} \cdot \mathrm{MeCN}$

$1029.5 \mathrm{~g} / \mathrm{mol}$

$3414(\mathrm{w}), 3125(\mathrm{w}), 2940(\mathrm{w}), 1624(\mathrm{w}), 1555(\mathrm{~m}), 1506(\mathrm{~s}), 1450$

(m), $1375(\mathrm{w}), 1287(\mathrm{w}), 1105(\mathrm{vs}), 985$ (m), $955(\mathrm{~m}), 845$ (m),

$757(\mathrm{~s}), 625(\mathrm{~s})$

MS (FAB, 3-NBA) $m / z(\%): \quad 807(25)\left[\mathrm{Cu}_{2} \mathrm{~L}^{4} \mathrm{Br}_{2}\left(\mathrm{ClO}_{4}\right)\right]^{+}, 789(35)\left[\mathrm{Cu}_{2} \mathrm{~L}^{4} \mathrm{Br}_{2}\right]^{+}, 708(100)$

$\left[\mathrm{Cu}_{2} \mathrm{~L}^{4} \mathrm{Br}\right]^{+}$

Elemental analysis (\%):

for $\mathrm{C}_{25} \mathrm{H}_{34} \mathrm{Cu}_{2} \mathrm{~N}_{12} \mathrm{O}_{8} \mathrm{Br}_{2} \mathrm{Cl}_{2}$

calcd.: C 30.38, H 3.47, N 17.00

found: C 30.45, H 3.66, N 16.90

$\mathrm{UV} / \mathrm{vis}(\mathrm{DMF}) \lambda(\mathrm{nm})[\varepsilon](\mathrm{L}$ $\left.\mathrm{mol}^{-1} \mathrm{~cm}^{-1}\right)$ :

$689(145)$ 


\subsection{Synthesis of 3,3',5,5'-tetramethylstilbene-4,4'-quinone (TMSQ)}



TMP

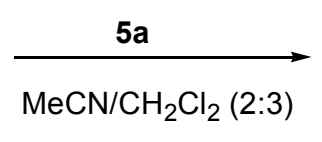

$\mathrm{MeCN} / \mathrm{CH}_{2} \mathrm{Cl}_{2}(2: 3)$



TMSQ

Complex 5a (0.05 g, $0.05 \mathrm{mmol})$ was dissolved in $\mathrm{MeCN} / \mathrm{CH}_{2} \mathrm{Cl}_{2}(10 \mathrm{~mL}$; ratio 2:3). The green solution was stirred for $10 \mathrm{~min}$ and then 2,4,6-trimethylphenol (TMP) (0.03 g, 0.25 mmol) was added. An immediate colour change of the reaction mixture from green to deepred was observed. The solution was left standing without stirring and after $16 \mathrm{~h}$ red crystals of the desired product TMSQ could be isolated.

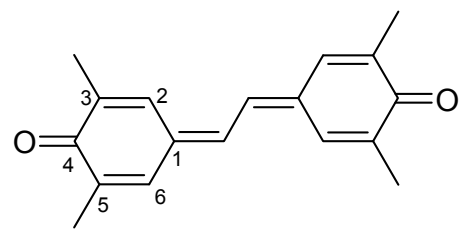

Yield:

$0.02 \mathrm{~g}, 66 \%$

Empirical formula: $\quad \mathrm{C}_{18} \mathrm{H}_{18} \mathrm{O}_{2}$

Molecular weight: $\quad 266.0 \mathrm{~g} / \mathrm{mol}$

${ }^{1} \mathrm{H}-\mathrm{NMR}\left(\mathrm{CDCl}_{3}\right) \delta(\mathrm{ppm}): \quad 2.05\left(\mathrm{~d}, J=1.3 \mathrm{~Hz}, 6 \mathrm{H}, \mathrm{CH}_{3}\right), 2.09\left(\mathrm{~d}, J=1.3 \mathrm{~Hz}, 6 \mathrm{H}, \mathrm{CH}_{3}\right), 7.01$ (s, 2H, $\left.\mathrm{C}^{2} \mathrm{H}\right), 7.19\left(\mathrm{~s}, 2 \mathrm{H}, \mathrm{C}^{6} \mathrm{H}\right), 7.51(\mathrm{~s}, 2 \mathrm{H}, \mathrm{CH})$

${ }^{13} \mathrm{C}-\mathrm{NMR}\left(\mathrm{CDCl}_{3}\right) \delta(\mathrm{ppm}): \quad 16.5\left(\mathrm{CH}_{3}\right), 17.1\left(\mathrm{CH}_{3}\right), 128.5,133.6,136.9,137.8,138.2,138.5$, $187.4(\mathrm{C}=\mathrm{O})$

MS (EI) $m / z(\%)$ :

$268(35)\left[\mathrm{C}_{18} \mathrm{H}_{18} \mathrm{O}_{2}+2 \mathrm{H}\right]^{+}, 266(100)\left[\mathrm{C}_{18} \mathrm{H}_{18} \mathrm{O}_{2}\right]^{+}, 251(38)$ $\left[\mathrm{C}_{18} \mathrm{H}_{18} \mathrm{O}+\mathrm{H}\right]^{+}, 236(15)\left[\mathrm{C}_{18} \mathrm{H}_{18}+\mathrm{H}\right]^{+}$

$\operatorname{IR}(\mathrm{KBr}) v\left(\mathrm{~cm}^{-1}\right)$ : 1637 (w), 1596 (vs), 1504 (w), 1435 (w), 1371 (w), 1094 (w), $1027(\mathrm{~m}), 941(\mathrm{~m})$

$\mathrm{UV} / \mathrm{vis}\left(\mathrm{CH}_{2} \mathrm{Cl}_{2}\right) \lambda(\mathrm{nm})[\varepsilon]$ 
$\left(\mathrm{L} \mathrm{mol}^{-1} \mathrm{~cm}^{-1}\right): \quad 440(96000)$

Elemental analysis (\%): $\quad$ for $\mathrm{C}_{18} \mathrm{H}_{18} \mathrm{O}_{2}$

calcd.: C 81.17, H 6.81

found: C 81.05, H 6.78

\subsection{Synthesis of 4,4'-dihydroxy-3,3',5,5'-tetramethylbibenzyl (TMBB)}

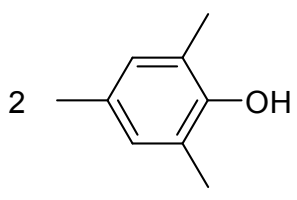

TMP

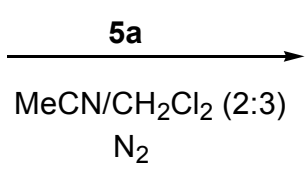

$\mathrm{N}_{2}$

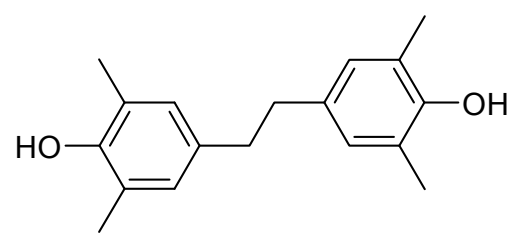

TMBB

Under strict anaerobic conditions, complex 5a (0.04 g, $0.05 \mathrm{mmol})$ was dissolved in $\mathrm{MeCN} / \mathrm{CH}_{2} \mathrm{Cl}_{2}$ (10 mL; ratio 2:3). The solution was stirred for $10 \mathrm{~min}$ and then TMP $(0.03 \mathrm{~g}$, $0.25 \mathrm{mmol}$ ) was added, which led to an immediate colour change of the reaction mixture from green to deep red. After $48 \mathrm{~h}$ of stirring in an $\mathrm{N}_{2}$-filled glove box, the colour of the solution had changed to yellow-green. Organic products were then separated from $\mathrm{Cu}$-containing species by chromatography on $\mathrm{SiO}_{2}$, using $\mathrm{CH}_{2} \mathrm{Cl}_{2} / \mathrm{MeOH}(9.5: 0.5)$ as eluent. The mixture of organic products was separated by HPLC chromatography (La Flash) with $\mathrm{CH}_{2} \mathrm{Cl}_{2} / \mathrm{MeOH}$ (9.5:0.5) as an eluent. Only TMP and TMBB were found to be present in the mixture after reaction. Solvent of the fraction containing only TMBB after separation by HPLC was evaporated and TMBB was isolated as a white powder.

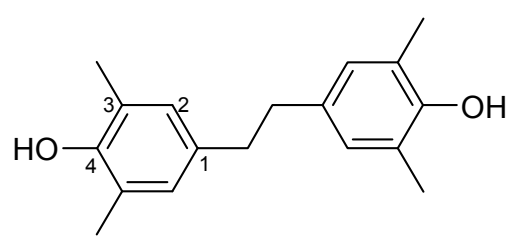

Yield:

$$
0.007 \text { g, 100\% }
$$


Empirical formula:

Molecular weight:

${ }^{1} \mathrm{H}-\mathrm{NMR}\left(\mathrm{CDCl}_{3}\right) \delta(\mathrm{ppm})$ :

${ }^{13} \mathrm{C}-\mathrm{NMR}\left(\mathrm{CDCl}_{3}\right) \delta(\mathrm{ppm})$ :

$\mathrm{MS}(\mathrm{EI}) m / z(\%)$ :

$$
\mathrm{C}_{18} \mathrm{H}_{22} \mathrm{O}_{2}
$$

$270.0 \mathrm{~g} / \mathrm{mol}$

$2.21\left(\mathrm{~s}, 12 \mathrm{H}, \mathrm{CH}_{3}\right), 2.70\left(\mathrm{~s}, 4 \mathrm{H}, \mathrm{CH}_{2}\right), 6.81(\mathrm{~s}, 4 \mathrm{H}, \mathrm{CH})$

$15.88\left(\mathrm{CH}_{3}\right), 37.66\left(\mathrm{CH}_{2}\right), 122.7\left(\mathrm{C}^{3}\right), 128.4\left(\mathrm{C}^{2} \mathrm{H}\right), 133.6\left(\mathrm{C}^{1}\right)$, $150.5\left(\mathrm{C}^{4}\right)$

$270(20)\left[\mathrm{C}_{18} \mathrm{H}_{22} \mathrm{O}_{2}\right]^{+}, 135(100)\left[\mathrm{C}_{9} \mathrm{H}_{11} \mathrm{O}\right]^{+}$ 


\subsection{Potentiometric titrations}

The $\mathrm{pH}$ potentiometric titrations were conducted at $25.0 \pm 0.1{ }^{\circ} \mathrm{C}$ at an ionic strength of $0.2 \mathrm{M}$ $(\mathrm{KCl})$ using a Radiometer PHM 84 pH-meter equipped with a Metrohm 6.0234.100 combined electrode and a Metrohm dosimat 715. Calibration of the electrode and pH-meter was performed using a buffer of potassium biphtalate at $\mathrm{pH} 4.008$. Concentrations of the stock solutions $\left(\mathrm{HCl} 0.2004 \mathrm{M}\right.$ and $\mathrm{KOH} 0.1972 \mathrm{M}$ ) were checked and a $\mathrm{p} K_{\mathrm{W}}$ of 13.784 and an Irving factor of 0.079 were obtained following Gran's method. ${ }^{180}$ Concentrations of the ligand stock solutions were also determined by Gran's method. The metal ion stock solution was prepared from $\mathrm{CuCl}_{2} \cdot 2 \mathrm{H}_{2} \mathrm{O}$ (Reanal) dissolved in doubly distilled water. The concentration of the metal ion stock solution was determined gravimetrically via precipitation with quinolin-8-olate.

$\mathrm{pH}$-metric titrations were performed in the $\mathrm{pH}$ range $2.0-10.5$ or until precipitation, on samples of $4.00 \mathrm{~mL}$, at an ionic strength of $0.2 \mathrm{M}(\mathrm{KCl})$ and at $25 \pm 0.1^{\circ} \mathrm{C}$. Purified, strictly oxygen-free argon was continuously bubbled through the samples during the titrations. The ligand concentrations were varied in the range $1 \cdot 10^{-3}-2 \cdot 10^{-3} \mathrm{M}$ and metal to ligand ratios were $1: 1,1.5: 1$ and $2: 1$.

The $\mathrm{pH}$-metric results were utilised to establish the stoichiometry of species and to calculate the stability constants. Calculations were performed with the computer programs SUPERQUAD and PSEQUAD ${ }^{181}$ while the speciation curves were created with the help of the MEDUSA program. ${ }^{182}$

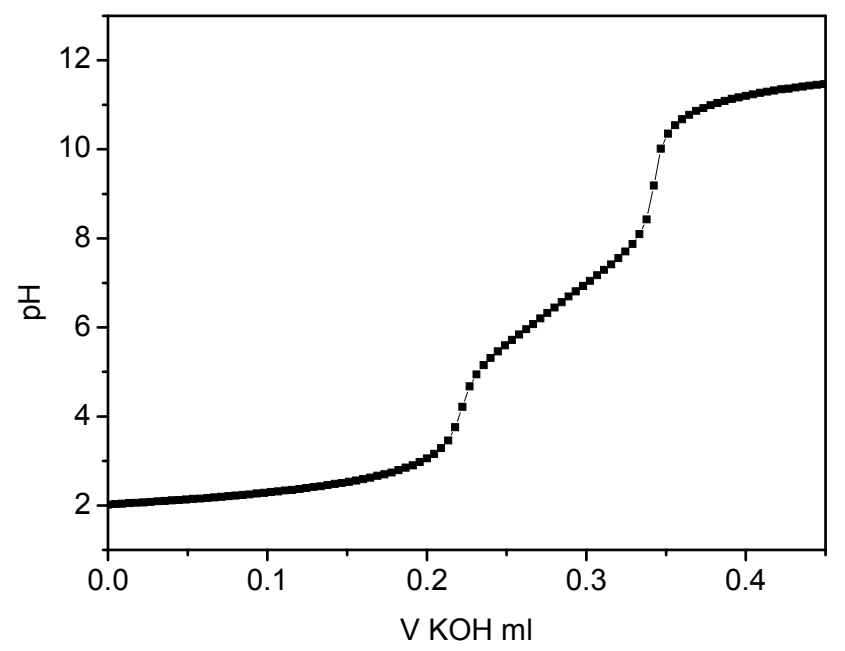

Figure 94. Titration curve of $\mathbf{H L}^{4}: \mathrm{C}_{\mathrm{HL}}{ }^{4}=1.5 \cdot 10^{-3} \mathrm{M}, \mathrm{C}_{\mathrm{KOH}}=0.1972 \mathrm{M}$. 


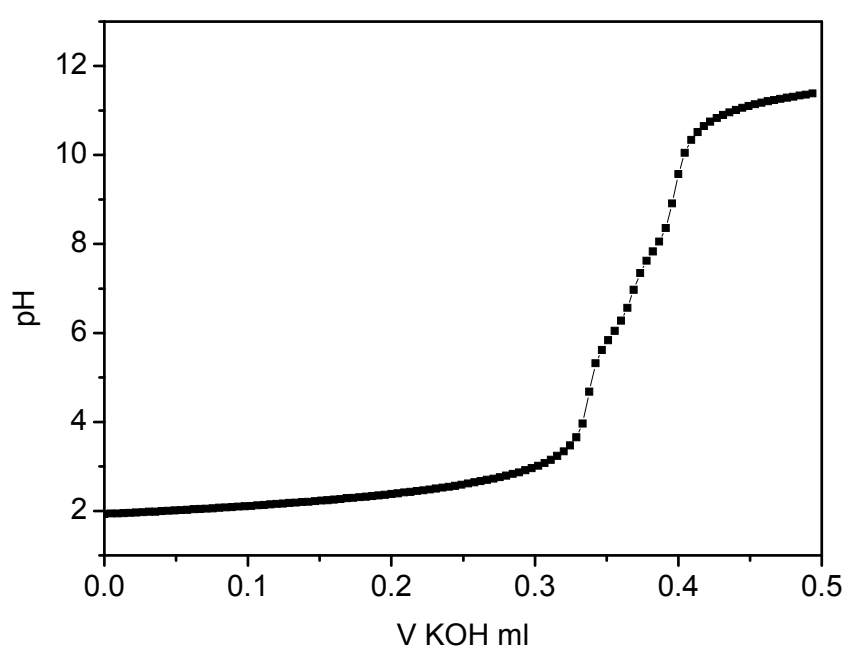

Figure 95. Titration curve of $\mathbf{H L}^{4}$ with 2 eq. $\mathrm{Cu}^{2+}: \mathrm{C}_{\mathrm{HL}}{ }^{4}=1.5 \cdot 10^{-3} \mathrm{M}, \mathrm{C}_{\mathrm{KOH}}=0.1972 \mathrm{M}$.

\subsection{X-ray crystallography}

X-ray data were collected on a STOE IPDS II diffractometer (graphite monochromated Mo$\mathrm{K} \alpha$ radiation, $\lambda=0.71073 \AA$ ) by use of $\omega$ scans at $-140{ }^{\circ} \mathrm{C}$ (Table 4$)$. The structures were solved by direct methods and refined on $F^{2}$ using all reflections with SHELX-97. ${ }^{183}$ Most of the non-hydrogen atoms were refined anisotropically. Unless otherwise noted, hydrogen atoms were placed in calculated positions and assigned to an isotropic displacement parameter of $0.08 \AA^{2}$. Face-indexed absorption corrections were performed numerically with the program X-RED. ${ }^{184} \mathrm{X}$-ray data for complex 15 were collected on a Bruker three-circle diffractometer with a graphite monochromator equipped with a SMART 6000 area detector $(\mathrm{Cu}-\mathrm{K} \alpha$ radiation, $\lambda=1.54184 \AA$ ). The crystal was cooled to $100 \mathrm{~K}$ with an Oxford Cryosystem. All calculations were carried out using SHELX software.

\section{Calculation of the $\tau$ parameter:}

In a five-co-ordinate system ideally square pyramidal geometry is associated with $\alpha=\beta=$ $180^{\circ}$, for $\mathrm{A}$ as the axial ligand ( $\beta$ is the greater of the basal angles, BMC, Figure 96). For perfectly trigonal bipyramidal geometry, $\alpha$ becomes $120^{\circ}$ and BMC the principal axis. In the great majority of real square pyramidal systems $\mathrm{M}$ is displaced out of the BCDE plane toward A, so that these $C_{4 v}$ geometries usually have $\alpha=\beta<180^{\circ}$, and can be characterised by the value of $(\beta-\alpha)$, which is $0^{\circ}$ for a $C_{4 v}$, and $60^{\circ}$ for a $D_{3 h}$ co-ordination polyhedron. 


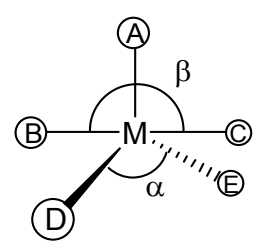

$\alpha=120^{\circ}$

$\beta=180^{\circ}$

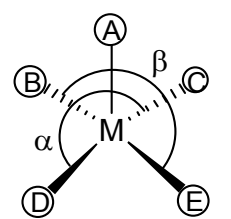

$\alpha=180^{\circ}$

$\beta=180^{\circ}$

Figure 96. Schematic representation of the $\tau$.

The geometric parameter $\tau$ is defined as $\tau=(\beta-\alpha) / 60$ and is applicable to five-co-ordinate structures as an index of the degree of trigonality, within the structural continuum between trigonal bipyramidal and rectangular pyramidal. For a perfectly tetragonal geometry $\tau$ is equal to zero, while it becomes one for a perfectly trigonal bipyramidal geometry. 


\subsection{Crystal data and refinement details}

\begin{tabular}{|c|c|c|}
\hline & 1 & 3 \\
\hline formula & $\mathrm{C}_{19} \mathrm{H}_{44} \mathrm{Cu}_{2} \mathrm{~N}_{6} \mathrm{O}_{3} \mathrm{~B}_{2} \mathrm{~F}_{8}$ & $\mathrm{C}_{50} \mathrm{H}_{106} \mathrm{Cu}_{2} \mathrm{~N}_{16} \mathrm{Cl}_{2} \mathrm{O}_{10} \cdot 0.47 \mathrm{H}_{2} \mathrm{O}$ \\
\hline $\mathrm{M}_{\mathrm{r}}$ & 705.3 & 1297.9 \\
\hline crystal size $\left[\mathrm{mm}^{3}\right]$ & $0.32 \times 0.28 \times 0.26$ & $0.22 \times 0.12 \times 0.11$ \\
\hline crystal system & Monoclinic & Triclinic \\
\hline space group & $P 2_{1}$ & $P-1$ \\
\hline$a[\AA]$ & $7.8856(5)$ & $12.3331(10)$ \\
\hline$b[\AA]$ & $12.2814(9)$ & $12.5231(10)$ \\
\hline$c[\AA]$ & $15.6430(8)$ & $12.6562(10)$ \\
\hline$\alpha\left[^{\circ}\right]$ & 90 & $62.598(6)$ \\
\hline$\beta\left[^{\circ}\right]$ & $99.926(4)$ & $72.108(6)$ \\
\hline$\gamma\left[^{\circ}\right]$ & 90 & $88.043(7)$ \\
\hline$V\left[\AA^{3}\right]$ & $1492.29(16)$ & $1638.1(2)$ \\
\hline$Z$ & 2 & 1 \\
\hline$\rho_{\text {calcd. }}\left[\mathrm{g} \mathrm{cm}^{-3}\right]$ & 1.570 & 1.316 \\
\hline$F(000)$ & 728 & 695 \\
\hline$\mu\left[\mathrm{mm}^{-1}\right]$ & 1.507 & 0.794 \\
\hline$h k l$ range & $\pm 9,-13$ to $14, \pm 18$ & -14 to $13, \pm 14, \pm 14$ \\
\hline$\theta$ range $\left[{ }^{\circ}\right]$ & $2.12-24.79$ & $1.85-24.79$ \\
\hline measured refl. & 16692 & 14859 \\
\hline unique refl. $\left[R_{\text {int }}\right]$ & $4691[0.0484]$ & $5604[0.0524]$ \\
\hline observed refl. $(I>2 \sigma(I))$ & 3865 & 4174 \\
\hline ref. param. / restraints & $373 / 2$ & $415 / 3$ \\
\hline goodness-of-fit & 1.013 & 1.003 \\
\hline$R 1, w R 2(I>2 \sigma(I))$ & $0.0543,0.1374$ & $0.0444,0.0965$ \\
\hline$R 1, w R 2$ (all data) & $0.0665,0.1448$ & $0.0678,0.1037$ \\
\hline resid. el. dens. $\left[\mathrm{e} \AA^{-3}\right]$ & $0.840 /-0.473$ & $0.297 /-0.542$ \\
\hline
\end{tabular}




\begin{tabular}{|c|c|c|}
\hline & $\mathbf{3 a}$ & $\mathbf{3 b}$ \\
\hline formula & $\mathrm{C}_{50} \mathrm{H}_{107} \mathrm{Cu}_{2} \mathrm{~N}_{16} \mathrm{Cl}_{3} \mathrm{O}_{15} \cdot 3 \mathrm{MeOH}$ & $\mathrm{C}_{50} \mathrm{H}_{106} \mathrm{Cu}_{2} \mathrm{~N}_{16} \mathrm{O}_{2} \mathrm{~B}_{2} \mathrm{~F}_{8}$ \\
\hline $\mathrm{M}_{\mathrm{r}}$ & 1486.1 & 1264.2 \\
\hline crystal size $\left[\mathrm{mm}^{3}\right]$ & $0.24 \times 0.20 \times 0.19$ & $0.23 \times 0.16 \times 0.12$ \\
\hline crystal system & Monoclinic & Triclinic \\
\hline space group & $P n$ & $P-1$ \\
\hline$a[\AA]$ & $14.9526(14)$ & $12.3153(10)$ \\
\hline$b[\AA]$ & $13.5704(8)$ & $12.3594(11)$ \\
\hline$c[\AA]$ & $17.9599(16)$ & $12.6747(10)$ \\
\hline$\alpha\left[^{\circ}\right]$ & 90 & $72.306(6)$ \\
\hline$\beta\left[^{\circ}\right]$ & $94.254(7)$ & $62.997(6)$ \\
\hline$\gamma\left[^{\circ}\right]$ & 90 & $88.085(7)$ \\
\hline$V\left[\AA^{3}\right]$ & $3634.3(5)$ & $1625.1(2)$ \\
\hline$Z$ & 2 & 1 \\
\hline$\rho_{\text {calcd. }}\left[\mathrm{g} \mathrm{cm}^{-3}\right]$ & 1.358 & 1.292 \\
\hline$F(000)$ & 1588 & 674 \\
\hline$\mu\left[\mathrm{mm}^{-1}\right]$ & 0.767 & 0.726 \\
\hline$h k l$ range & $\pm 17,-14$ to $15,-21$ to 18 & $\pm 14, \pm 14, \pm 14$ \\
\hline$\theta$ range $\left[{ }^{\circ}\right]$ & $1.50-24.78$ & $1.74-24.77$ \\
\hline measured refl. & 17359 & 17951 \\
\hline unique refl. $\left[R_{\text {int }}\right]$ & $11052[0.0745]$ & $5537[0.0561]$ \\
\hline observed refl. $(I>2 \sigma(I))$ & 7168 & 4106 \\
\hline ref. param. / restraints & $810 / 190$ & $362 / 52$ \\
\hline goodness-of-fit & 1.017 & 1.032 \\
\hline$R 1, w R 2(I>2 \sigma(I))$ & $0.0782,0.1685$ & $0.0686,0.1779$ \\
\hline$R 1, w R 2$ (all data) & $0.1270,0.1953$ & $0.0967,0.1943$ \\
\hline resid. el. dens. $\left[\mathrm{e} \AA^{-3}\right]$ & $0.696 /-0.463$ & $1.521 /-0.628$ \\
\hline
\end{tabular}




\begin{tabular}{|c|c|c|}
\hline & $5 \mathbf{a}$ & $\mathbf{5 b}$ \\
\hline formula & $\mathrm{C}_{27} \mathrm{H}_{40} \mathrm{Cu}_{2} \mathrm{~N}_{12} \mathrm{Cl}_{2} \mathrm{O}_{10} \cdot \mathrm{MeCN}$ & $\mathrm{C}_{27} \mathrm{H}_{40} \mathrm{Cu}_{2} \mathrm{~N}_{14} \mathrm{O}_{8} \cdot 2 \mathrm{MeOH}$ \\
\hline $\mathrm{M}_{\mathrm{r}}$ & 931.7 & 879.9 \\
\hline crystal size $\left[\mathrm{mm}^{3}\right]$ & $0.46 \times 0.24 \times 0.21$ & $0.40 \times 0.23 \times 0.12$ \\
\hline crystal system & Monoclinic & Triclinic \\
\hline space group & $C c$ & $P-1$ \\
\hline$a[\AA]$ & $18.1991(9)$ & $11.1984(4)$ \\
\hline$b[\AA]$ & $16.9395(6)$ & $14.9118(6)$ \\
\hline$c[\AA]$ & $13.6063(8)$ & $24.3582(9)$ \\
\hline$\alpha\left[^{\circ}\right]$ & 90 & $101.663(3)$ \\
\hline$\beta\left[^{\circ}\right]$ & $112.350(4)$ & $99.134(3)$ \\
\hline$\gamma\left[^{\circ}\right]$ & 90 & $100.299(3)$ \\
\hline$V\left[\AA^{3}\right]$ & $3879.5(3)$ & $3838.4(3)$ \\
\hline$Z$ & 4 & 4 \\
\hline$\rho_{\text {calcd. }}\left[\mathrm{g} \mathrm{cm}^{-3}\right]$ & 1.595 & 1.523 \\
\hline$F(000)$ & 1920 & 1832 \\
\hline$\mu\left[\mathrm{mm}^{-1}\right]$ & 1.305 & 1.180 \\
\hline$h k l$ range & $\pm 21, \pm 19,-16$ to 15 & $\pm 13,-15$ to $17, \pm 28$ \\
\hline$\theta$ range $\left[{ }^{\circ}\right]$ & $1.71-24.81$ & $1.43-24.79$ \\
\hline measured refl. & 29113 & 57759 \\
\hline unique refl. $\left[R_{\mathrm{int}}\right]$ & $6527[0.0651]$ & $13096[0.0591]$ \\
\hline observed refl. $(I>2 \sigma(I))$ & 6123 & 9972 \\
\hline ref. param. / restraints & $553 / 4$ & $1010 / 36$ \\
\hline goodness-of-fit & 1.001 & 1.017 \\
\hline$R 1, w R 2(I>2 \sigma(I))$ & $0.0287,0.0674$ & $0.0520,0.1262$ \\
\hline$R 1, w R 2$ (all data) & $0.0314,0.0682$ & $0.0743,0.1367$ \\
\hline resid. el. dens. $\left[\mathrm{e} \AA^{-3}\right]$ & $0.351 /-0.275$ & $1.439 /-0.743$ \\
\hline
\end{tabular}




\begin{tabular}{|c|c|c|}
\hline & 6 & 7 \\
\hline Formula & $\mathrm{C}_{50} \mathrm{H}_{80} \mathrm{Cu}_{4} \mathrm{~N}_{30} \mathrm{O}_{25} \cdot 5 \mathrm{H}_{2} \mathrm{O}$ & $\mathrm{C}_{25} \mathrm{H}_{38} \mathrm{Cl}_{4} \mathrm{Cu}_{2} \mathrm{~N}_{12} \mathrm{O}_{18} \cdot 2 \mathrm{H}_{2} \mathrm{O}$ \\
\hline$M_{r}$ & 1755.6 & 1099.6 \\
\hline crystal size $\left[\mathrm{mm}^{3}\right]$ & $0.50 \times 0.26 \times 0.21$ & $0.50 \times 0.08 \times 0.07$ \\
\hline crystal system & Triclinic & Orthorhombic \\
\hline space group & $P-1$ & Pbca \\
\hline$a[\AA]$ & $9.5728(6)$ & $18.1999(8))$ \\
\hline$b[\AA]$ & $13.1348(8)$ & $17.0313(5)$ \\
\hline$c[\AA]$ & $14.5660(9)$ & $26.7503(9)$ \\
\hline$\alpha\left[^{\circ}\right]$ & $94.003(5)$ & 90 \\
\hline$\beta\left[^{\circ}\right]$ & $106.635(5)$ & 90 \\
\hline$\gamma\left[^{\circ}\right]$ & $93.850(5)$ & 90 \\
\hline$V\left[\AA^{3}\right]$ & $1743.26(19)$ & $8291.7(5)$ \\
\hline$Z$ & 1 & 8 \\
\hline$\rho_{\text {calcd. }}\left[\mathrm{g} \mathrm{cm}^{-3}\right]$ & 1.672 & 1.762 \\
\hline$F(000)$ & 906 & 4496 \\
\hline$\mu\left[\mathrm{mm}^{-1}\right]$ & 1.305 & 1.377 \\
\hline$h k l$ range & $\pm 11, \pm 15,-15$ to 17 & $\pm 21,-17$ to $20,-31$ to 27 \\
\hline$\theta$ range $\left[{ }^{\circ}\right]$ & $1.56-24.74$ & $1.81-24.89$ \\
\hline measured refl. & 27830 & 78080 \\
\hline unique refl. $\left[R_{\mathrm{int}}\right]$ & $5954[0.0554]$ & $7173[0.1116]$ \\
\hline observed refl. $(I>2 \sigma(I))$ & 5232 & 4914 \\
\hline ref. param. / restraints & $563 / 38$ & $627 / 58$ \\
\hline goodness-of-fit & 1.040 & 1.005 \\
\hline$R 1, w R 2(I>2 \sigma(I))$ & $0.0312,0.0836$ & $0.0481,0.1100$ \\
\hline$R 1, w R 2$ (all data) & $0.0364,0.0860$ & $0.0806,0.1211$ \\
\hline resid. el. dens. $\left[\mathrm{e} \AA^{-3}\right]$ & $0.439 /-0.533$ & $0.828 /-0.490$ \\
\hline
\end{tabular}




\begin{tabular}{|c|c|c|}
\hline & 8 & 9 \\
\hline Formula & $\mathrm{C}_{62} \mathrm{H}_{76} \mathrm{Cu}_{4} \mathrm{~N}_{28} \mathrm{O}_{14} \cdot 0.5 \mathrm{MeCN}$ & $\mathrm{C}_{70} \mathrm{H}_{102} \mathrm{Cu}_{4} \mathrm{~N}_{28} \mathrm{Cl}_{4} \mathrm{O}_{16} \cdot 7 \mathrm{MeCN}$ \\
\hline$M_{r}$ & 1712.2 & 2275.5 \\
\hline crystal size $\left[\mathrm{mm}^{3}\right]$ & $0.49 \times 0.33 \times 0.21$ & $0.30 \times 0.24 \times 0.19$ \\
\hline crystal system & Triclinic & Triclinic \\
\hline space group & $P-1$ & $P-1$ \\
\hline$a[\AA]$ & $15.5078(5)$ & $10.8800(7)$ \\
\hline$b[\AA]$ & $15.9822(6)$ & $14.0868(9)$ \\
\hline$c[\AA]$ & $19.2030(7)$ & $18.5825(12)$ \\
\hline$\alpha\left[^{\circ}\right]$ & $109.753(3)$ & $100.300(5)$ \\
\hline$\beta\left[^{\circ}\right]$ & $95.324(3)$ & $102.835(5)$ \\
\hline$\gamma\left[^{\circ}\right]$ & $92.625(3)$ & $96.127(5)$ \\
\hline$V\left[\AA^{3}\right]$ & $4444.9(3)$ & $2700.1(3)$ \\
\hline$Z$ & 2 & 1 \\
\hline$\rho_{\text {calcd. }}\left[\mathrm{g} \mathrm{cm}^{-3}\right]$ & 1.279 & 1.473 \\
\hline$F(000)$ & 1766 & 1244 \\
\hline$\mu\left[\mathrm{mm}^{-1}\right]$ & 1.013 & 0.955 \\
\hline$h k l$ range & $\pm 18, \pm 18, \pm 22$ & $\pm 12, \pm 16, \pm 21$ \\
\hline$\theta$ range $\left[{ }^{\circ}\right]$ & $1.32-24.82$ & $1.68-24.76$ \\
\hline measured refl. & 120620 & 27741 \\
\hline unique refl. $\left[R_{\text {int }}\right]$ & $15215[0.0626]$ & $9163[0.0517]$ \\
\hline observed refl. $(I>2 \sigma(I))$ & 11535 & 6981 \\
\hline ref. param. / restraints & $978 / 86$ & $617 / 0$ \\
\hline goodness-of-fit & 1.057 & 1.031 \\
\hline$R 1, w R 2(I>2 \sigma(I))$ & $0.0579,0.1684$ & $0.0546,0.1469$ \\
\hline$R 1, w R 2$ (all data) & $0.0744,0.1773$ & $0.0739,0.1559$ \\
\hline resid. el. dens. $\left[\mathrm{e} \AA^{-3}\right]$ & $1.136 /-0.870$ & $0.594 /-1.399$ \\
\hline
\end{tabular}




\begin{tabular}{|c|c|c|}
\hline & 11 & 12 \\
\hline Formula & $\mathrm{C}_{32} \mathrm{H}_{437} \mathrm{Cl}_{2} \mathrm{Cu}_{2} \mathrm{~F}_{5} \mathrm{~N}_{12} \mathrm{O}_{10} \cdot \mathrm{MeOH}$ & $\mathrm{C}_{33} \mathrm{H}_{43} \mathrm{Cu}_{2} \mathrm{~N}_{15} \mathrm{O}_{9} \cdot 4 \mathrm{MeOH}$ \\
\hline$M_{r}$ & 1074.7 & 1049.1 \\
\hline crystal size $\left[\mathrm{mm}^{3}\right]$ & $0.22 \times 0.17 \times 0.13$ & $0.50 \times 0.11 \times 0.07$ \\
\hline crystal system & Triclinic & Orthorhombic \\
\hline space group & $P-1$ & Pbca \\
\hline$a[\AA]$ & $11.5528(11)$ & $14.7719(5)$ \\
\hline$b[\AA]$ & $13.1929(14)$ & $23.6254(8)$ \\
\hline$c[\AA]$ & $14.9182(14)$ & $27.7160(14)$ \\
\hline$\alpha\left[^{\circ}\right]$ & $93.767(8)$ & 90 \\
\hline$\beta\left[^{\circ}\right]$ & $100.704(8)$ & 90 \\
\hline$\gamma\left[^{\circ}\right]$ & $93.458(8)$ & 90 \\
\hline$V\left[\AA^{3}\right]$ & $2223.3(4)$ & $9672.7(7)$ \\
\hline$Z$ & 2 & 8 \\
\hline$\rho_{\text {calcd. }}\left[\mathrm{g} \mathrm{cm}^{-3}\right]$ & 1.605 & 1.441 \\
\hline$F(000)$ & 1096 & 4384 \\
\hline$\mu\left[\mathrm{mm}^{-1}\right]$ & 1.167 & 0.954 \\
\hline$h k l$ range & -11 to $13, \pm 15, \pm 17$ & -15 to $17, \pm 27, \pm 32$ \\
\hline$\theta$ range $\left[{ }^{\circ}\right]$ & $1.55-24.77$ & $1.47-24.77$ \\
\hline measured refl. & 21952 & 70349 \\
\hline unique refl. $\left[R_{\text {int }}\right]$ & $7560[0.0973]$ & 8244 [0.0999] \\
\hline observed refl. $(I>2 \sigma(I))$ & 4877 & 6307 \\
\hline ref. param. / restraints & $607 / 62$ & $619 / 72$ \\
\hline goodness-of-fit & 1.007 & 1.113 \\
\hline$R 1, w R 2(I>2 \sigma(I))$ & $0.0696,0.1776$ & $0.1110,0.2784$ \\
\hline$R 1, w R 2$ (all data) & $0.1100,0.2023$ & $0.1353,0.2919$ \\
\hline resid. el. dens. $\left[\mathrm{e} \AA^{-3}\right]$ & $0.933 /-0.652$ & $1.768 /-1.677$ \\
\hline
\end{tabular}




\begin{tabular}{|c|c|c|}
\hline & 13 & 14 \\
\hline formula & $\mathrm{C}_{60} \mathrm{H}_{78} \mathrm{Cu}_{4} \mathrm{~N}_{30} \mathrm{Cl}_{4} \mathrm{O}_{16}$ & $\mathrm{C}_{74} \mathrm{H}_{80} \mathrm{Cu}_{5} \mathrm{~N}_{26} \mathrm{Cl}_{14} \mathrm{O}_{16}$ \\
\hline $\mathrm{M}_{\mathrm{r}}$ & 1871.4 & 2403.6 \\
\hline crystal size $\left[\mathrm{mm}^{3}\right]$ & $0.50 \times 0.37 \times 0.25$ & $0.21 \times 0.18 \times 0.15$ \\
\hline crystal system & Triclinic & Monoclinic \\
\hline space group & $P-1$ & $C 2 / c$ \\
\hline$a[\AA]$ & $14.9304(6)$ & $13.5632(7)$ \\
\hline$b[\AA]$ & $15.3395(6)$ & $31.5050(15)$ \\
\hline$c[\AA]$ & $19.4766(8)$ & $30.5438(17)$ \\
\hline$\alpha\left[^{\circ}\right]$ & $96.812(3)$ & 90 \\
\hline$\beta\left[^{\circ}\right]$ & $90.133(3)$ & $96.195(4)$ \\
\hline$\gamma\left[^{\circ}\right]$ & $113.409(3)$ & 90 \\
\hline$V\left[\AA^{3}\right]$ & $4058.6(3)$ & $12975.4(12)$ \\
\hline$Z$ & 2 & 4 \\
\hline$\rho_{\text {calcd. }}\left[\mathrm{g} \mathrm{cm}^{-3}\right]$ & 1.531 & 1.230 \\
\hline$F(000)$ & 1920 & 4868 \\
\hline$\mu\left[\mathrm{mm}^{-1}\right]$ & 1.246 & 1.151 \\
\hline$h k l$ range & $\pm 19, \pm 19, \pm 25$ & -11 to $15, \pm 37,-36$ to 35 \\
\hline$\theta$ range $\left[{ }^{\circ}\right]$ & $1.49-27.41$ & $1.29-24.84$ \\
\hline measured refl. & 80546 & 37794 \\
\hline unique refl. $\left[R_{\text {int }}\right]$ & $18316[0.0456]$ & $10803[0.0671]$ \\
\hline observed refl. $(I>2 \sigma(I))$ & 13711 & 6149 \\
\hline ref. param. / restraints & $1089 / 155$ & $615 / 0$ \\
\hline goodness-of-fit & 1.018 & 1.001 \\
\hline$R 1, w R 2(I>2 \sigma(I))$ & $0.0437,0.1203$ & $0.0592,0.1398$ \\
\hline$R 1, w R 2$ (all data) & $0.0612,0.1272$ & $0.0976,0.1511$ \\
\hline resid. el. dens. [e $\left.\AA^{-3}\right]$ & $1.205 /-0.672$ & $0.483 /-0.643$ \\
\hline
\end{tabular}




\begin{tabular}{|c|c|}
\hline & 17 \\
\hline formula & $\mathrm{C}_{25} \mathrm{H}_{34} \mathrm{Cu}_{2} \mathrm{~N}_{12} \mathrm{Br}_{2} \mathrm{Cl}_{2} \mathrm{O}_{8} \cdot \mathrm{MeCN}$ \\
\hline $\mathrm{M}_{\mathrm{r}}$ & 1029.5 \\
\hline crystal size $\left[\mathrm{mm}^{3}\right]$ & $0.50 \times 0.11 \times 0.07$ \\
\hline crystal system & Monoclinic \\
\hline space group & $P 2_{1} / n$ \\
\hline$a[\AA]$ & $21.9846(12)$ \\
\hline$b[\AA]$ & $7.8001(2)$ \\
\hline$c[\AA]$ & $24.1070(17)$ \\
\hline$\alpha\left[^{\circ}\right]$ & 90 \\
\hline$\beta\left[^{\circ}\right]$ & $115.222(5)$ \\
\hline$\gamma\left[^{\circ}\right]$ & 90 \\
\hline$V\left[\AA^{3}\right]$ & $3739.8(3)$ \\
\hline$Z$ & 4 \\
\hline$\rho_{\text {calcd. }}\left[\mathrm{g} \mathrm{cm}^{-3}\right]$ & 1.828 \\
\hline$F(000)$ & 2064 \\
\hline$\mu\left[\mathrm{mm}^{-1}\right]$ & 3.483 \\
\hline$h k l$ range & $\pm 25,-9$ to $18, \pm 28$ \\
\hline$\theta$ range $\left[{ }^{\circ}\right]$ & $1.65-24.79$ \\
\hline measured refl. & 49669 \\
\hline unique refl. $\left[R_{\mathrm{int}}\right]$ & $6390[0.0957]$ \\
\hline observed refl. $(I>2 \sigma(I))$ & 4082 \\
\hline ref. param. / restraints & $483 / 119$ \\
\hline goodness-of-fit & 1.039 \\
\hline$R 1, w R 2(I>2 \sigma(I))$ & $0.0671,0.1567$ \\
\hline$R 1, w R 2$ (all data) & $0.1096,0.1760$ \\
\hline resid. el. dens. $\left[\mathrm{e} \AA^{-3}\right]$ & $1.510 /-1.369$ \\
\hline
\end{tabular}




\section{Literature}

Klausener A., Jentsch J.D. In: Cornils B, Herrmann WA (eds) Applied Homogeneous Catalysis with Organometallic Compounds, VCH, Weinheim 1, 169, 1996.

(a) Kaim W., Rall J. Angew. Chem. Int. Ed. 1996, 35, 43; (b) Malmström B.G., Leckner J. Curr. Opin. Chem. Biol. 1998, 2, 286; (c) Pascaly M., Jolk I., Krebs B. Chemie in unsere Zeit 1999, 33, 334.

Carey F.A., Sundberg R.J. Organische Chemie, VCH, Weinheim, 1, 1995.

Streitwieser A., Heathcock C.H., Kosower E.M. Organische Chemie, VCH, 2, Weinheim 1994.

(a) Smidt J., Hafner W., Jira R., Sedlmeier J., Rüttinger R. Consortium für Elektrochemische Industrie DE 1049 845, 1959, (b) Jira R., Blau W., Grimm D. Hydrocarbon Processing55, 97, 1975.

Jira R. In: Cornils R, Herrmann WA (eds) Applied Homogeneous Catalysis with Organometallic Compounds, VCH, Weinheim 1996, 374., ibid 2002 1, 386.

(a) Naworski J.S., Velez E.S. In: Leach B.A. (ed) Applied Industrial Catalysis, Academic Press, New York, 239, 1983; (b) Spector M.L., Heinemann H., Miller K.D. Ind. Eng. Chem. Process Res. Dev. 6, 1967, 327.

Hathaway B.J. in Comprehensive Coordination Chemistry, 5, Pergamon, New York, 1987.

Jameson R.F. Met. Ions Biol. Syst. 1981, 12, 1.

Nigh W.G. Oxidation in Organic Chemistry, Part B, Trahanovsky W.S. Ed., Academie, New York, 1, 1973.

Koval I. A., Gamez P., Belle C., Selmeczi K., Reedijk J. Chem. Soc. Rev. 2006, 35, 814.

(a) Steinhagen H., Helmchen G. Angew. Chem. Int. Ed. 1996, 35, 2339; (b) Fenton D.E., Okawa H. Chem. Ber./Recueil 1997, 130, 433; (c) Van den Beuken E.K., Feringa B.L. Tetrahedron 1998, 54, 12985; (d) Bosnich B. Inorg. Chem. 1999, 38, 2554; (e) Belle C., Pierre J.-L. Eur. J. Inorg. Chem. 2003, 4137.

Klingele J., Dechert S., Meyer F. Coord. Chem. Rev. submitted.

Schenck T.G., Downes J.M., Milne C.R.C., Mackenzie P.B., Boucher H., Whelan J., Bosnich B. Inorg. Chem. 1985, 24, 2334.

(a) Meyer F., Beyreuther S., Heinze K., Zsolnai L. Chem. Ber./Recueil 1997, 130, 605;

(b) Meyer F., Heinze K., Nuber B., Zsolnai L. J. Chem. Soc., Dalton Trans. 1998, 
207-213; (c) Konrad M., Meyer F., Heinze K., Zsolnai, L. J. Chem. Soc., Dalton Trans. 1998, 199; (d) Buchler S., Meyer F., Jacobi A., Kircher P., Zsolnai L. Z. Naturforsch. B 1999, 54, 1295; (e) Konrad M., Wuthe S., Meyer F., Kaifer E. Eur. J. Inorg. Chem. 2001, 2233; (f) Buchler S., Meyer F., Kaifer E., Pritzkow H. Inorg. Chim. Acta 2002, 337, 371; (g) Röder J.C., Meyer F., Kaifer E., Pritzkow H. Eur. J. Inorg. Chem. 2004, 1646; (h) Ackermann J., Meyer F., Pritzkow H. Inorg. Chim. Acta 2004, 357, 3703-3711.

Siegfried L., Kaden T.A., Meyer, F., Kircher P., Pritzkow H. J. Chem. Soc., Dalton Trans. 2001, 2310.

(a) Kamiusuki T., Okawa H., Matsumoto N., Kida S. J. Chem. Soc., Dalton Trans. 1990, 195; (b) Mernari B., Abraham F., Lagrenee M., Drillon M., Legoli P. J. Chem. Soc., Dalton Trans. 1993, 1707; (c) Weller H., Siegfried L., Neuburger M., Zehnder M., Kaden T.A. Helv. Chim. Acta 1997, 80, 2315; (d) Kaden T.A. Coord. Chem. Rev. 1999, 190-192, 371; (e) Tanaka S., Dubs C., Inagaki A., Akita M. Organometallics 2005, 24, 163; (f) Miranda C., Escarti F., Lamarque L., Garcia-Espana E., Navarro P., Latorre J., Lloret F., Jimenez H.R., Yunta M.J.R. Eur. J. Inorg. Chem. 2005, 189; (g) Zinn P.J., Powell D.R., Day V.W., Hendrich M.P., Sorrell T.N., Borovik A.S. Inorg. Chem. 2006, 45, 3484.

Kaim W., Schwederski B. Bioanorganische Chemie, Teubner Studienbücher, Stuttgart, 1991.

Voet D., Voet J.G., Pratt C.W. Lehrbuch der Biochemie, Wiley-VCH, Weinheim, 2002.

Gerdemann C., Eicken C., Krebs B. Acc. Chem. Res. 2002, 35, 183.

Gaykema W.P.J., Hol W.G.J., Vereijken J.M., Soeter N.M., Bak H.J., Beintema J.J. Nature 1984, 309, 23.

Magnus K.A., Thon-Tat H., Carpenter J.E. Chem. Rev. 1994, 94, 727.

Cuff M.E., Miller K.I., van Holde K.E., Hendrickson W.A. J. Mol. Biol. 1998, 278, 855.

Magnus K.A., Hazes B., Thon-Tat H., Bonaventura C., Bonaventura J., Hol W.G.J. Proteins : Structure, Function and Genetics 1994, 19, 302.

(a) Ito N., Phillips S.E.V., Stevens C., Ogel Z.B, McPherson M.J., Keen J.N., Yadav K.D.S., Knowles P.F. Nature 1991, 350, 87; (b) Ito N., Phillips S.E.V., Yadav K.D.S., Knowles P.F.J. Mol Biol. 1994, 238, 794.

Klinman J.P. Chem. Rev. 1996, 96, 2541. 
(a) Eisensmith R.C., Woo S.L.C. Mol. Biol. Med. 1991, 8, 3; (b) Fitzpatrick P.F. Biochemistry 2003, 42,14083.

(a) Tainer J.A., Getzoff E.D., Beem K.M., Richardson J.S., Richardson D.C. J. Mol. Biol. 1982, 160, 181; (b) Tainer J.A., Getzoff E.D., Richardson J.S., Richardson D.C. Nature 1983, 306, 284.

Solomon E.I., Baldwin M.J., Lowery M.D. Chem. Rev. 1992, 92, 521.

(a) Volbeda A., Hol W.G.J. J. Mol. Biol. 1989, 206, 531; (b) Volbeda A., Hol W.G.J. J Mol. Biol. 1989, 209, 249.

Matoba Y., Kumagai T., Yamamoto A., Yoshitsu H., Sugiyama M. J. Biol. Chem. 2006, 31, 8981.

Klabunde T., Eicken C., Sacchettini J.C., Krebs B. Nat. Struct. Biol. 1998, 5, 1084.

(a) Eicken C., Krebs B., Sacchettini J.C. Curr. Opin. Struct. Biol. 1999, 9, 677; (b) Gerdemann C., Eicken C., Krebs B. Acc. Chem. Res. 2002, 183.

Siegbahn P.E.M. J. Biol. Inorg. Chem. 2004, 9, 577.

Decker H., Schweikardt T., Tuczek F. Angew. Chem. Int. Ed. 2006, 45, 4546.

(a) Decker H., Dillinger R., Tuczek F. Angew. Chem. Int. Ed. 2000, 39, 1591; (b) Siegbahn P.E.M. J. Biol. Inorg. Chem. 2003, 8, 567; (c) Granata A., Monzani E., Bubacco L., Casella L. Chem. Eur. J. 2006, 12, 2504; (d) Cramer C.J., Wloch M., Piecuch P., Puzzarini C., Gagliardi L. J. Phys. Chem. A. 2006, 110, 1991.

(a) Bertrand T., Jolivalt C., Briozzo P., Caminade E., Joly N., Madzak C., Mougin C. Biochemistry 2002, 41, 7325; (b) Piontec K., Antorini M., Choinowski T. J. Biol. Chem. 2002, 277, 37663; (c) Claus H. Micron 2004, 35, 93.

Hakulinen N., Kiiskinen L.L., Kruus K., Saloheimo M., Paananen A., Koivula A. Rouvinen J. Nat. Struct. Biol. 2002, 9, 601.

Messerschmidt A., Rossi A., Ladenstein R., Huber R., Bolognesi M., Gatti G., Machesini A., Petruzzelli R., Finazzi-Agró A. J. Mol. Biol. 1989, 206, 513.

Wang Y., DuBois J., Hedman B., Hodgson K., Stack T. Science 1998, $279,537$.

Chaudhuri P., Hess M., Weyhermüller T., Wieghardt K. Angew. Chem. Int. Ed. 1999, $38,1095$.

Karlin K.D., Hayes J.C., Gultneh Y., Cruse R.W., McKown J.W., Hutchinson J.P., Zubieta J. J. Am. Chem. Soc. 1984, 106, 2121.

Karlin K.D., Kaderli S., Zuberbühler A.D. Acc. Chem. Res. 1997, 30, 139.

Santagostini L., Gullotti M., Monzani E., Casella L., Dillinger R., Tuczek F. Chem. Eur. J. 2000, 6, 519. 
Monzani E., Quinti L., Perotti A., Casella L., Gullotti M., Randaccio L., Geremia S., Nardin G., Faleschini P., Tabbi G. Inorg. Chem. 1998, 37, 553.

Monzani E., Battaini G., Perotti A., Casella L., Gullotti M., Santagostini L., Randaccio L., Geremia S., Nardin G., Zanello P., Opromolla G. Inorg. Chem. 1999, 38, 5359.

Casella L., Monzani E., Gullotti M., Cavagnino D., Cerina G., Santagostini L., Ugo R. Inorg. Chem. 1996, 35, 7516.

Karlin K.D., Zubieta J. Copper Coordination Chemistry: Biochemical and Inorganic Perspectives, Adenine, New York, 1983.

Karlin K.D., Hayes J.C., Juen S., Hutchinson J.P., Zubieta J. Inorg. Chem. 1982, 21, 4106.

Kleywegt G.J., Wiesmeijer W.G.R., van Dreil G.J., Driessen W.L., Reedijk J., Noordik J.H. J. Chem. Soc., Dalton Trans. 1985, 2177.

Karlin K.D., Dahlstrom P.L., Hayes J.C., Simon R.A., Zubieta J. Cryst. Struct. Commun. 1982, 11, 907.

Sorrell T.N., Jameson D.L. Inorg. Chem. 1982, 21, 1014.

Berreau L.M. Eur. J. Inorg. Chem. 2006, 273.

(a) Schindler S. Eur. J. Inorg. Chem. 2000, 2311; (b) Würtele C., Gaoutchenova E., Harms K., Holthausen M.C., Sundermeyer J., Schindler S. Angew. Chem. Int. Ed. 2006, 45, 3867.

(a) Jacobson R.J., Tyeklar Z., Farooq A., Karlin K.D., Liu S., Zubieta J. J. Am. Chem. Soc. 1988, 110, 3690; (b) Wei N., Murthy N.N., Tyeklar Z., Karlin K.D. Inorg. Chem. 1994, 33, 1177; (b) Schatz M., Becker M., Thaler F., Hampel F., Schindler S., Jacobson R.R., Tyeklar Z., Murthy N.N., Ghosh P., Chen Q., Zubieta J., Karlin K. Inorg. Chem. 2001, 40, 2312; (c) Uozumi K., Hayashi Y., Suzuki M., Uehara A. Chem. Lett. 1993, 963; (d) Komiyama K., Furutachi H., Nagatomo S., Hashimoto A., Hayashi H., Fujinami S., Suzuki M., Kitagawa T. Bull. Chem. Soc. Jpn. 2004, 77, 59.

Bol J.E., Driessen W.L., Ho A.Y.N., Maase B., Que L., Reedijk J. Angew. Chem. Int. Ed. 1997, 36, 998.

Bode R.H., Bol J.E., Driessen W.L., Hulsbergen F.B., Reedijk J., Spek A.L. Inorg. Chem. 1999, 38, 1239. van der Vlugt J.I., Meyer F. Top. Organomet. Chem. 2007, 22, 191.

(a) Mirica L.M., Vance M., Rudd D.J., Hedman B., Hodgson K.O., Solomon E.I., Stack T.D.P. J. Am. Chem. Soc. 2002, 124, 9332; (b) Mirica L.M., Rudd D.J., Vance 
M.A., Solomon E.I., Hodgson K.O., Hedman B., Stack T.D.P. J. Am. Chem. Soc. 2006, 128, 2654.

Mahadevan V., DuBois J.L., Hedman B., Hodgson K.O., Stack T.D.P. J. Am. Chem. Soc. 1999, 121, 5583.

(a) Santagostini L., Gullotti M., Monzani E., Casella L., Dillinger R., Tuczek F. Chem. Eur. J. 2000, 6, 529; (b) Battaini G, de Carolis M., Monzani E., Tuczek F., Casella L. Chem. Commun. 2003, 726; (c) Palavicini S., Granata A., Monzani E., Casella L. J. Am. Chem. Soc. 2005, 127, 18031.

Itoh S., Kumei H., Taki M., Nagatomo S., Kitagawa T., Fukuzumi S. J. Am. Chem. Soc. 2001, 123, 6708.

Monzani E., Quinti L., Perotti A., Casella L., Gullotti M., Randaccio L., Geremia S., Nardin G., Faleschini P., Tabbì G. Inorg. Chem. 1998, 37, 553.

Mirica L.M., Vance M., Rudd D.J., Hedman B., Hodgson K.O., Solomon E.I., Stack T.D.P. Science 2005, 308, 1890.

Mukherjee R. N. Proc. Indian. Natn. Sci. Acad. 2004, 70, 329.

Monzani E., Battaini G., Perotti A., Casella L., Gullotti M., Santagostini L., Nardin G., Randaccio L., Geremia S., Zanello P., Opromolla G. Inorg. Chem. 1999, 38, 5359.

(a) Selmeczi K., Réglier M., Giorgi M., Speier G. Coord. Chem. Rev. 2003, 245, 191;

(b) Kaizer J., Csonka R., Speier G., Giorgi M., Réglier M. J. Mol. Catal. A: Chem. 2005, 235,81 .

Selmeczi K., Réglier M., Speier G., Peintler G. React. Kinet. Catal. Lett. 2004, 81, 143 Granata A., Monzani E., Casella L. J. Biol. Inorg. Chem. 2004, 9, 903.

Koval I.A., Belle C., Selmeczi K., Philouze C., Saint-Aman E., Schuitema A.M., Gamez P., Pierre J.L., Reedijk J. J. Biol. Inorg. Chem. 2005, 10, 739.

Ackermann J., Meyer F., Kaifer E., Pritzkow H. Chem. Eur. J. 2002, 8, 247.

Kao C.H., Wei H.H., Liu Y.H., Lee G.H., Wang Y., Lee C.J. J. Inorg. Biochem. 2001, $84,171$.

Malachowski M.R. Inorg. Chim. Acta 1989, 162, 199.

Ackermann J., Buchler S., Meyer F. C. R. Chimie 2006, 10, 421.

(a) Oishi N., Nishida Y., Ida K., Kida S. Bull. Chem. Soc. Jpn. 1980, 53, 2847; (b) Mukherjee J., Mukherjee R. Inorg. Chim. Acta 2002, 337, 429.

Kao C.H., Wei H.H., Liu Y.H., Lee G.H., Wang Y., Lee C.J. J. Inorg. Biochem. 2001, 84, 171. 
(a) Torelli S., Belle C., Gautier-Luneau I., Pierre J.L., Saint-Aman E., Latour J.M., Le Pape L., Luneau D. Inorg. Chem. 2000, 39, 3526; (b) Belle C., Beguin C., GautierLuneau I., Hamman S., Philouze C., Pierre J.L., Thomas F., Torelli S., Saint-Aman E., Bonin M. Inorg. Chem. 2002, 41, 479.

(a) Reim J., Krebs B. J. Chem. Soc., Dalton. Trans. 1997, 3793; (b) Anekwe J., Hammerschmidt A., Rompel A., Krebs B. Z. anorg. allg. Chem. 2006, 632, 1057.

Merkel M., Möller N., Piacenza M., Grimme S., Rompel A., Krebs B. Chem. Eur. J. 2005, 11, 1201.

Thirumavalavan M., Akilan P., Kandaswamy M., Chinnakali K., Senthil Kumar G., Fun H.K. Inorg. Chem. 2003, 42, 3308.

(a) Meyer F., Heinze K., Nuber B., Zsolnai L. J. Chem. Soc., Dalton Trans. 1998, 207;

(b) Meyer F., Rutsch P. Chem. Commun. 1998, 1037.

Baesjou P.J., Driessen W.L., Challa G., Reedijk J. J. Mol. Catal. A: Chem. 1996, 110, 195.

(a) Hay A.S. J. Polym. Sci., Part A: Polym. Chem. 1998, 36, 505; (b) Gamez P., Simons C., Steensma R., Driessen W.L, Challa G., Reedijk J. Eur. Polym. J. 2001, 37, 1293; (c) Gamez P., Simons C., Aromi G., Driessen W.L, Challa G., Reedijk J. Appl. Catal. A 2001, 214, 187.

Gamez P., van Dijk J.A.P.P., Driessen W.L., Challa G., Reedijk J. Adv. Synth. Catal. 2002, 344, 890 .

Saito K., Tago T., Masuyama T., Nishide H. Angew. Chem. Int. Ed. 2004, 43, 730. Ullmann R. F. Chem. Ber. 1904, 37, 853.

Saawyer J.S., Schmittling E.A., Palkowitz J.A., Smith W.J. J. Org. Chem. 1998, 63, 6338.

Gupta R., Mukherjee R. Tetrahedron Lett. 2000, 41, 7763.

Kodera M., Shimakoshi H., Tachi Y., Katayama K., Kano K. Chem. Lett. 1998, 27, 441.

(a) Schuchardt U., Cardoso D., Sercheli R., Pereira R., da Cruz R.S., Guerreiro M.C., Mandelli D., Spinacé E.V. and Pires E.L. Appl. Catal. A 2001, 211, 1; (b) Sheldon R.A. and Kochi J.K. in Metal-Catalyzed Oxidations of Organic Compounds, Academic Press, New York, 1981.

(a) Shimokawa C., Yokota S., Tachi Y., Nishiwaki N., Ariga M., Itoh S. Inorg. Chem. 2003, 42, 8395; (b) Shimokawa C., Teraoka J., Tachi Y., Itoh S. J. Inorg. Biochem. 2006, 100,1118 . 
Lieberman R.L., Rosenzweig A.C. Nature 2005, 434, 177.

(a) Murahashi S.I., Oda Y., Naota T., Komiya N. J. Chem. Soc., Chem. Commun. 1993, 139; (b) Komiya N., Naota T., Murahashi S.I. Tetrahedron Lett. 1996, 37, 1633. (a) Kirillov A.M., Kopylovich M.N., Kirillova M.V., Haukka M., Guedes da Silva M.F.C., Pombeiro A.J.L. Angew. Chem. Int. Ed. 2005, 44, 4345; (b) Kirillov A.M., Kopylovich M.N., Kirillova M.V., Karabach E.Y.; Haukka M., Guedes da Silva M.F.C., Pombeiro A.J.L. Adv. Synth. Catal. 2006, 348, 159.

Costas M., Llobet A. J. Mol. Cat. A: Chem. 1999, 142, 113.

(a) Takehira K., Shimizu M., Watanabe Y., Orita H., Hayakawa T. Tetrahedron Lett. 1990, 31, 2607; (b) Shimizu M., Watanabe Y., Orita H., Hayakawa T., Takehira K. Tetrahedron Lett. 1991, 32, 2053; (c) Shimizu M., Watanabe Y., Orita H., Hayakawa T. Bull. Chem. Soc. Jpn. 1993, 66, 251.

van den Heuvel R.H.H., Fraaije M.W., Ferrer M., Mattevi A., van Berkel W.J.H. Proc. Natl. Acad. Sci. USA 2000, 97, 9455.

Boldron C., Gamez P., Tooke D.M., Spek A.L., Reedijk J. Angew. Chem. Int. Ed. 2005, 44, 3585 .

Boldron C., Özalp-Yaman S., Gamez P., Tooke D.M., Spek A.L., Reedijk J. Dalton Trans. 2005, 21, 3535.

Schenck T.G., Downes J.M., Milne C.R.C., MacKenzie P.B., Boucher H., Whelan J., Bosnich B. Inorg. Chem. 1985, 24, 2334.

Bradshaw J.S., Nielsen R.B., Tse P., Arena G., Wilson B.E., Dalley N.K., Lamb J.D., Christensen J.C., Izatt R.M. J. Heterocycl. Chem. 1986, 23, 361.

Röder J.C., Meyer F., Pritzkow H. Organometallics 2001, 20, 811.

(a) Nagao H., Komeda N., Mukaida M., Suzuki M.,Tanaka K. Inorg. Chem. 1996, 35, 6809; (b) Oberhausen K.J., Richardson, J.F., Buchanan R.M., Pierce W. Polyhedron 1989, 8, 659; (c) Rodriguez M.C., Morgenstern-Badarau I., Cesario M.,Guilhem J., Keita B., Nadjo L. Inorg. Chem. 1996, 35, 7804.

(a) Myhre P.C., Maxey C.T., Bebout D.C., Swedberg S.H., Petersen B.L. J. Org. Chem. 1990, 55, 3417; (b) first fully developed in the group of Prof. F. Meyer by Anna Sachse.

105 Buchner E., van der Heide C. Berichte d.D.chem.Gesellschaft 1901, 31.

106 Addison A.W., Rao T.N., Reedijk J., Van Rijn J., Verschoor G.C. J. Chem. Soc., Dalton. Trans. 1984, 1349.

unpublished results by Ackermann J., Gamez P., Reedijk J., Meyer F. 
Ackermann J. Dissertation, Georg-August University Göttingen, 2003.

Buijs W., Comba P., Corneli D., Pritzkow H. J. Organomet. Chem. 2002, 641, 71.

Xin Zhang C., Liang H.C., Kim E., Gan O.F., Tyeklár Z., Karlin K.D., Lam K.C., Rheingold A.L., Kaderli S., Zuberbühler A.D. Chem. Commun. 2001, 631.

Alvariño Gil M. Dissertation, Georg-August University Göttingen, 2006.

unpublished results by Nie F.-M.

Ackermann J., Meyer F., Pritzkow H. Inorg. Chim. Acta 2004, 357, 3703.

Chiu Y.-H., Canary J.W. Inorg. Chem. 2003, 42, 5107.

Oberhausen K.J., O’Brien R.J., Richardson J.F., Buchanan R.M. Inorg. Chim. Acta 1990, 173, 145.

Lever A.B.P. Inorganic Electronic Spectroscopy, Elsevier, 1984.

(a) Meyer F., Pritzkow H. Chem. Commun. 1998, 1555; (b) Meyer F., Kaifer E., Kircher P., Heinze K., Pritzkow, H. Chem. Eur. J. 1999, 5, 1617; (c) Meyer F., HylaKryspin I., Kaifer E., Kircher P. Eur. J. Inorg. Chem. 2000, 771; (d) Kryatov S.V., Rybak-Akimova E.V., Meyer F., Pritzkow H. Eur. J. Inorg. Chem. 2003, 1581; (e) Bauer-Siebenlist B., Meyer F., Farkas E., Vidovic D., Seijo J.A.C., Herbst-Irmer R., Pritzkow H. Inorg. Chem. 2004, 43, 4189; (f) Bauer-Siebenlist B., Meyer F., Farkas E., Vidovic D., Dechert S. Chem. Eur. J. 2005, 11, 4349; (g) Bauer-Siebenlist B., Dechert S., Meyer F. Chem. Eur. J. 2005, 11, 5343.

Kahn O. Molecular Magnetism, Wiley-VCH, Publishers Inc., 1993.

Simulation of the experimental magnetic data with a full-matrix diagonalisation of exchange coupling and Zeeman splitting was performed with the julX program: E. Bill, Max-Planck Institute for Bioinorganic Chemistry, Mülheim/Ruhr, Germany.

(a) Meyer F., Jacobi A., Zsolnai L. Chem. Ber./Recueil 1997, 130, 1441; (b) Teichgräber J., Leibeling G., Dechert S., Meyer F. Z. anorg. allg. Chem. 2005, 631, 2613.

Bayoñ J.C., Esteban P., Net G., Rasmussen P.G., Baker K.N., Hahn C.W., Gumz M.M. Inorg. Chem. 1991, 30, 2572.

Kamiusuki T., Okawa H., Matsumoto N., Kida S. J. Chem. Soc., Dalton Trans. 1990, 195.

Matsushima H., Hamada H., Watanabe K., Koikawa M., Tokii T. J. Chem. Soc., Dalton Trans. 1999, 971.

Ajò D., Bencini A., Mani F. Inorg. Chem. 1988, 27, 2437. 
Hanot V.P., Robert T.D., Kolnaar J., Haasnoot J.P., Reedijk J., Kooijman H., Spek A.L. J. Chem. Soc., Dalton Trans. 1996, 4275.

Boldron C., Aromí G., Challa G., Gamez P., Reedijk J. Chem. Commun. 2005, 5808.

Balogh V., Fetizon M., Golfier M. J. Org. Chem. 1971, 36, 1339.

Oyaizu K., Saito K., Tsuchida E. Chem. Lett. 2000, 1318.

Butte Jr.,W.A., Price C.C. J. Am. Chem. Soc. 1962, 84, 3567.

Jackman L.M., Chen X. J. Am. Chem. Soc. 1997, 119, 8681.

Harrod J.F. Can. J. Chem. 1969, 47, 637.

Jazdzewski B.A., Holland P.L., Pink M., Young V.G., Jr. Spencer D.J.E., Tolman W.B. Inorg. Chem. 2001, 40, 6097.

Kitajima N., Koda T., Iwata Y., Morooka Y. J. Am. Chem. Soc. 1990, 112, 8833.

Baesjou P.J., Driessen W.L., Challa G., Reedijk J. J. Am. Chem. Soc. 1997, 119, 12590.

Cosgrove S.L., Waters W.A. J. Chem. Soc. 1951, 388.

Walling C., Hodgon R.B., Jr. J. Am. Chem. Soc. 1958, 80, 228.

Auerbach U., Eckert U., Wieghardt K., Nuber B., Weiss J. Inorg. Chem. 1990, 29, 938.

Sokolowski A., Leutbecher H., Weyhermüller T., Schnepf R., Bothe E., Bill E., Hildebrandt P., Wieghardt K. J. Biol. Inorg. Chem. 1997, 2, 444.

Halfen J.A., Jazdzewski B.A., Mahapatra S., Berreau L.M., Wilkinson E.C., Que L., Jr., Tolman W.B. J. Am. Chem. Soc. 1997, 35, 8217.

Schnepf R., Sokolowski A., Müller J., Bachler V., Wieghardt K., Hildebrandt P. J. Am. Chem. Soc. 1998, 120, 2352.

Hockertz J., Steenken S., Wieghardt K., Hildebrandt P. J. Am. Chem. Soc. 1993, 115, 11222

Müller J., Weyhermüller T., Bill E., Hildebrandt P., Ould-Moussa L., Glaser T., Wieghardt K. Angew. Chem. Int. Ed. 1998, 37, 616.

Sokolowski A., Müller J., Weyhermüller T., Schnepf R., Hildebrandt P., Hildenbrand K., Bothe E., Wieghardt K. J. Am. Chem. Soc. 1997, 119, 8889.

(a) Sokolowski A., Adam B., Weyhermüller T., Kikuchi A., Hildenbrand K., Schnepf R., Hildebrandt P., Bill E., Wieghardt K. Inorg. Chem. 1997, 36, 3702; (b) Snodin M.D., Ould-Moussa L., Wallman U., Lecomte S., Bachler V., Bill E., Hummel H., Weyhermüller T., Hildebrandt P., Wieghardt K. Chem. Eur. J. 1999, 5, 2554.

Mukherjee A., McGlashen M.L., Spiro T.G. J. Phys. Chem. 1995, 99, 4912. 
146

(a) Tripathi G.N.R., Schuler R.H. J. Phys. Chem. 1988, 92,5129; (b) Qin Y., Wheeler R.A. J. Am. Chem. Soc. 1995, 117, 6083.

Pyrz J.W., Karlin K.D., Sorrell T.N., Vogel G.C., Que L., Jr. Inorg. Chem. 1984, 23, 4581.

Bill E., Müller J., Weyhermüller T., Wieghardt K. Inorg. Chem. 1999, 38, 5795.

Altwicker E.R. Chem. Rev. 1967, 67, 475.

Job P. Ann. Chim. 1928, 9, 113.

Wada A., Honda Y., Yamaguchi S., Nagamoto S., Kitagawa T., Jitsukawa K., Masuda H. Inorg. Chem. 2004, 43. 5725.

Ardizzoia G.A., Angaroni M.A., La Monica G., Masciocchi N., Moret M. J. Chem. Soc., Dalton Trans, 1990, 2277.

Ardizzoia G.A., Beccalli E.M., La Monica G., Masciocchi N., Moret M. Inorg. Chem. 1992, 31, 2706.

Ardizzoia G.A., Cenini S., La Monica G., Masciocchi N., Moret M. Inorg. Chem. 1994, 33, 1458.

Dias H.V.R., Lu H.L., Gorden J.D., Jin W. Inorg. Chem. 1996, 35, 2149.

(a) Knowles P.F., Ito N. In Perspectives in Bioinorganic Chemistry; Jai Press: London, 1994, 2, 207; (b) Whittaker J.W., Whittaker M.M. Pure Appl. Chem. 1998, $70,903$.

Solomon E.I., Sundaram U.M., Machonkin T.E. Chem. Rev. 1996, 96, 2563.

(a) Wang Y., DuBois J.L., Hedman B., Hodgson K.O., Stack T.D.P. Science 1998, 279, 537; (b) Chaudhuri P., Hess M., Flörke U., Wieghardt K. Angew. Chem., Int. Ed. 1998, 37, 2217; (c) Chaudhuri P., Hess M., Weyhermüller T., Wieghardt K. Angew. Chem., Int. Ed. 1999, 38, 1095; (d) Chaudhuri P., Hess M., Müller J., Hildenbrand K., Bill E., Weyhermüller T., Wieghardt K. J. Am. Chem. Soc. 1999, 121, 9599.

(a) Hay A.S. J. Polym. Sci., Part A: Polym. Chem. 1998, 36, 505; (b) Higashimura H., Kubota M., Shiga A., Fujisawa K., Morooka Y., Uyama H., Kobayashi S. Macromolecules 2000, 33, 1986.

Jazdzewski B.A., Tolman W.B. Coord. Chem. Rev. 2000, 200-202, 633.

(a) Bullock J.I., Hobson R.J., Povey D.C. J. Chem. Soc., Dalton Trans. 1974, 2037;

(b) Calderazzo F., Marchetti F., Dell'Amico G., Pelizzi G., Colligiani A. J. Chem. Soc., Dalton Trans. 1980, 1419; (c) Whittaker M., Chuang Y., Whittaker J. J. Am. Chem. Soc. 1993, 115, 10029. 
(a) Karlin K.D., Cohen B.I.,. Hayes J.C,. Farooq A, Zubieta J. Inorg. Chem. 1987, 26, 147; (b) Rajendran U., Viswanathan R., Palaniandavar M., Lakshminarayanan M. J. Chem. Soc., Dalton. Trans. 1992, 3563; (c) Uma R., Viswanathan R., Palaniandavar M., Lakshminarayanan M. J. Chem. Soc., Dalton Trans. 1994, 1219; (d) Adams H., Bailey N.A., Barbarin C.O.R.D., Fenton D.E., He Q.-Y. J. Chem. Soc., Dalton Trans. 1995, 2323; (e) Adams H., Bailey N.A., Campbell I.K., Fenton D.E., He Q.-Y. J. Chem. Soc., Dalton Trans. 1996, 2233; (f) Ito S., Nishino S., Itoh H., Ohba S., Nishida Y. Polyhedron 1998, 17, 1637.

(a) Karlin K.D., Cohen B.I. Inorg. Chim. Acta 1985, 107, L17; (b) Adams H., Bailey N.A., Fenton D.E., He Q., Ohba M., Okawa H. Inorg. Chim. Acta 1994, 215 1; (c) Itoh S., Takayama S., Arakawa R., Furuta A., Komatsu M., Ishida A., Takamuku S., Fukuzumi S. Inorg. Chem. 1997, 36, 1407; (d) Itoh S., Taki M., Takayama S., Nagatomo S., Kitagawa T., Sakurada N., Arakawa R., Fukuzumi S. Angew. Chem. Int. Ed. 1999, 38, 2774.

Que L., Jr. In Biological Applications of Raman Spectroscopy; Wiley: New York, 3, 1988, 491.

Pyrz J. W., Roe A. L., Stern L. J., Que L., Jr. J. Am. Chem. Soc. 1985, 107, 614.

Li L., Sarjeant A.A.N., Vance M.A., Zakharov L.N., Rheingold A.L., Solomon E.I., Karlin K.D. J. Am. Chem. Soc. 2005, 127, 15360.

(a) Lever A.B.P., Ramaswamy B.S., Pickens S.R. Inorg. Chim. Acta 1980, 46, L5961; (b) Demmin T.R., Swerdloff M.D., Rogic M.M. J. Am. Chem. Soc. 1981, 103, 5795; (c) Bolus D., Vigee G.S. Inorg. Chim. Acta 1982, 67, 19; (d) Speier G.J. Mol. Cat. 1986, 37, 259; (e) Réglier M., Jorand C., Waegell B. J. Chem. Soc., Chem. Commun. 1990, 1752; (f) Chyn J.-P., Urbach F.L. Inorg. Chim. Acta 1991, 189, 157; (g) Malachowski M.R., Tomlinson L.J., Davidson M.G., Hall M.J. J. Coord. Chem. 1992, 25, 171; (h) Rockcliffe D.A., Martell A.E. J. Mol. Catal. A 1995, 99, 101; (i) Zippel F., Ahlers F., Werner R., Haase W., Nolting H.-F., Krebs B. Inorg. Chem. 1996, 35, 3409; (j) Chung Y.-H., Wei H.-H., Liu Y.-H., Lee G.-H., Wang Y. J. Chem. Soc., Dalton Trans. 1997, 2825; (k) Manzur J., Garcia A.M., Rivas V., Atria A.M., Valenzuela J., Spodine E. Polyhedron 1997, 16, 2299.

168 Malachowski M.R., Huynh H.B., Tomlinson L.J., Kelly R.S., Jr., Furbee J.W. J. Chem. Soc., Dalton Trans. 1995, 31. 
(a) Casellato U., Tamburini S., Vigato P.A., de Stefani A., Vidali M., Fenton D.E. Inorg. Chim. Acta 1983, 69, 45; (b) Malachowski M.R., Davidson M.G. Inorg. Chim. Acta 1989, 162, 199.

(a) Karlin K.D., Gultneh Y., Nicholson T., Zubieta J. Inorg. Chem. 1985, 24, 3725;

(b) Börzel H., Comba P., Pritzkow H. Chem. Commun. 2001, 97.

Berreau L.M., Mahapatra S., Halfen J.A., Houser R.P., Young V.G., Tolman W.B. Angew. Chem. Int. Ed. 1999, 38, 207.

Song Y.-F., van Albada G.A., Tang J., Mutikainen I., Turpeinen U., Massera C., Roubeau O., Costa J.S., Gamez P., Reedijk J. Inorg. Chem. 2007, 46, 4944.

(a) Sahoo S.K., Liu W., Samuelson L.A., Kumar J., Cholli A.L. Macromolecules 2002, 35, 9990; (b) Asakura K., Honda E., Osanai S. Chem.Lett. 1995, 583.

Gleason J.G., Holden K.G., Nelson C.F.Y. US Pat. 1975, 3,860,631.

Gong Y.F., Kato K., Kimoto H. Bull. Chem. Soc. Jpn. 2002, 75, 2637.

Yan J.M., Zhang Z.J., Yuan D.Q., Xie R.G., Zhao H.M. Synth. Commun. 1994, 24, 47. Habata Y., Akabori S. J. Chem. Soc., Dalton Trans. 1996, 3871.

Itoh T., Nakanishi E., Okayama M., Kubo M. Macromolecules 2000, 33, 269.

Gamez P., Gupta S., Reedijk J. C. R. Chimie 2007, 10, 295.

Gran G. Analyst 1952, 77, 661.

(a) Gans P., Sabatini A., Vacca A. J. Chem. Soc., Dalton Trans. 1985, 1195; (b) Zékány L., Nagypál I. in: D.L. Leggett (Ed.), Computational Methods for the Determination of Stability Constants, Plenum Press, New York, 1985, 291.

Puigdomenech I. MEDUSA and Hydra software for chemical equilibrium calculations, Royal Institute of Technology (KTH), Stockholm, Sweden.

Sheldrick G.M. SHELXL-97, Program for Crystal Structure Refinement, University of Göttingen, Göttingen (Germany), 1997. Sheldrick, G.M. SHELXS-97, Program for Crystal Structure Solution, University of Göttingen, Göttingen (Germany), 1997. STOE \& CIE GmbH, X-RED, Darmstadt, 2002. 




$H L^{1}$

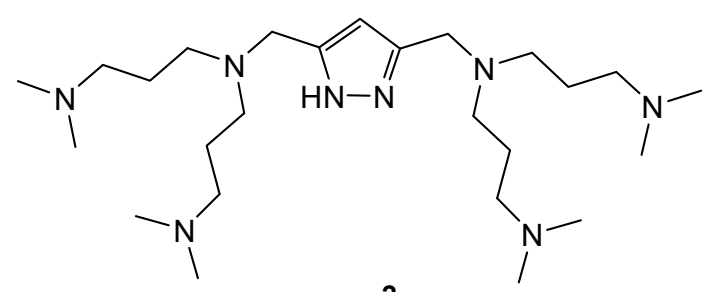

$\mathrm{HL}^{2}$<smiles>Cc1cccc(CN(Cc2cc(CN(Cc3cccc(C)n3)Cc3cccc(C)n3)[nH]n2)Cc2cccc(C)n2)n1</smiles>

$\mathrm{HL}^{3}$

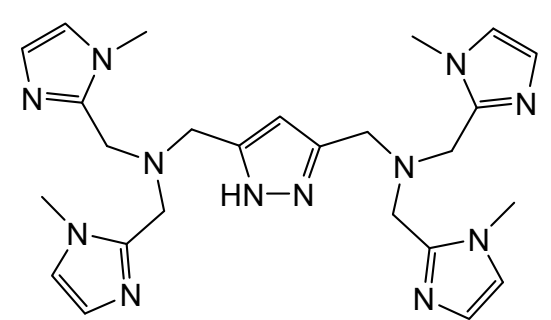

$\mathrm{HL}^{4}$

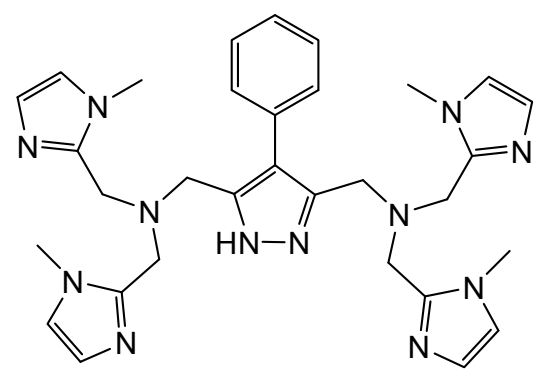

$\mathrm{HL}^{5}$ 


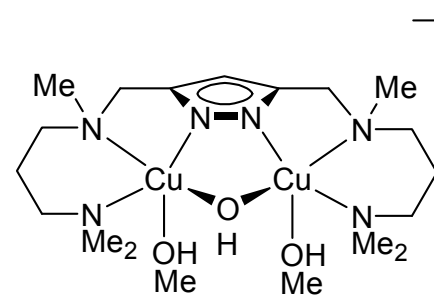

1

$\left(\mathrm{BF}_{4}^{-}\right)_{2}$

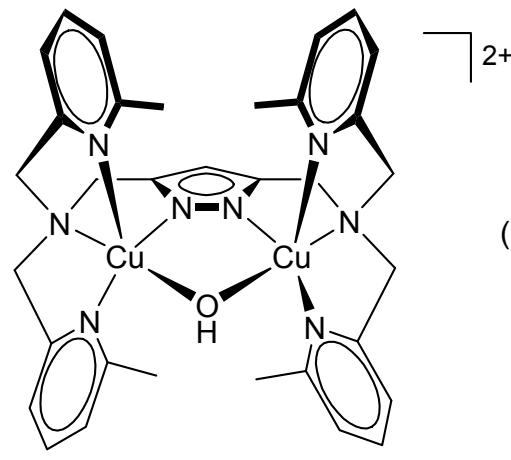

4

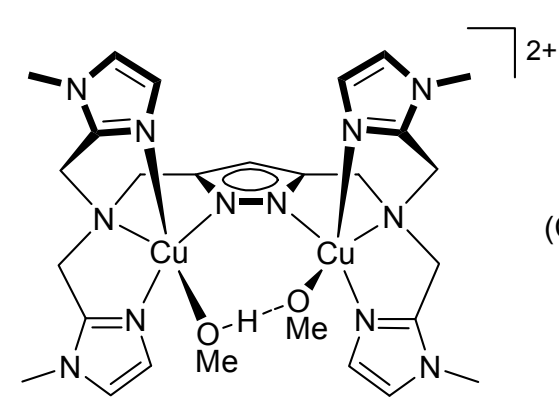

$5 a$


$3 a$

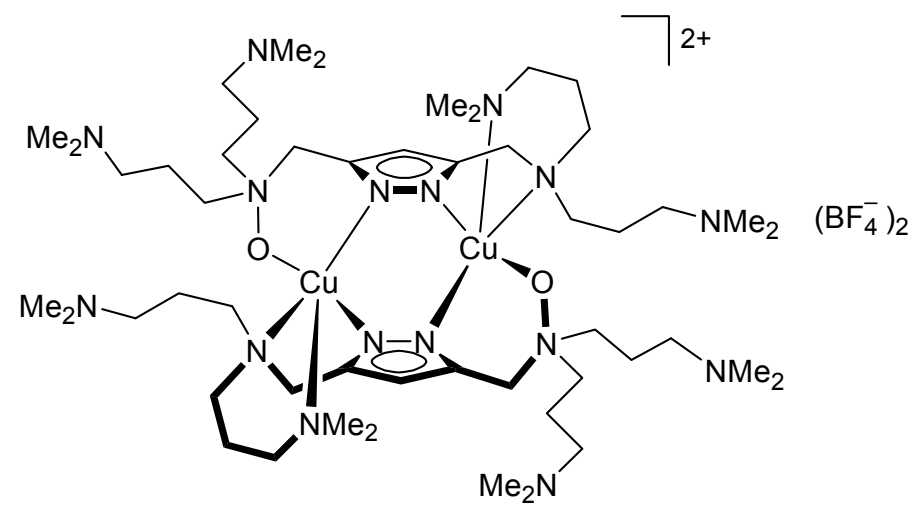

$3 b$ 


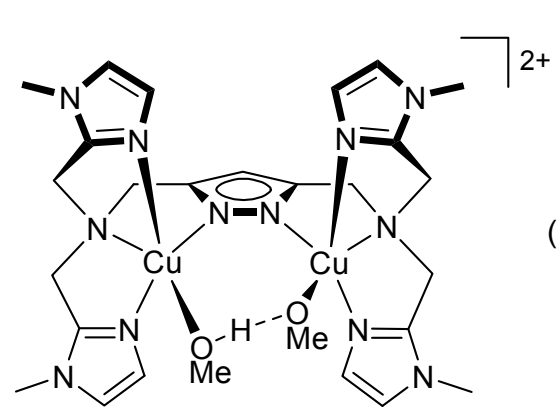

$5 b$
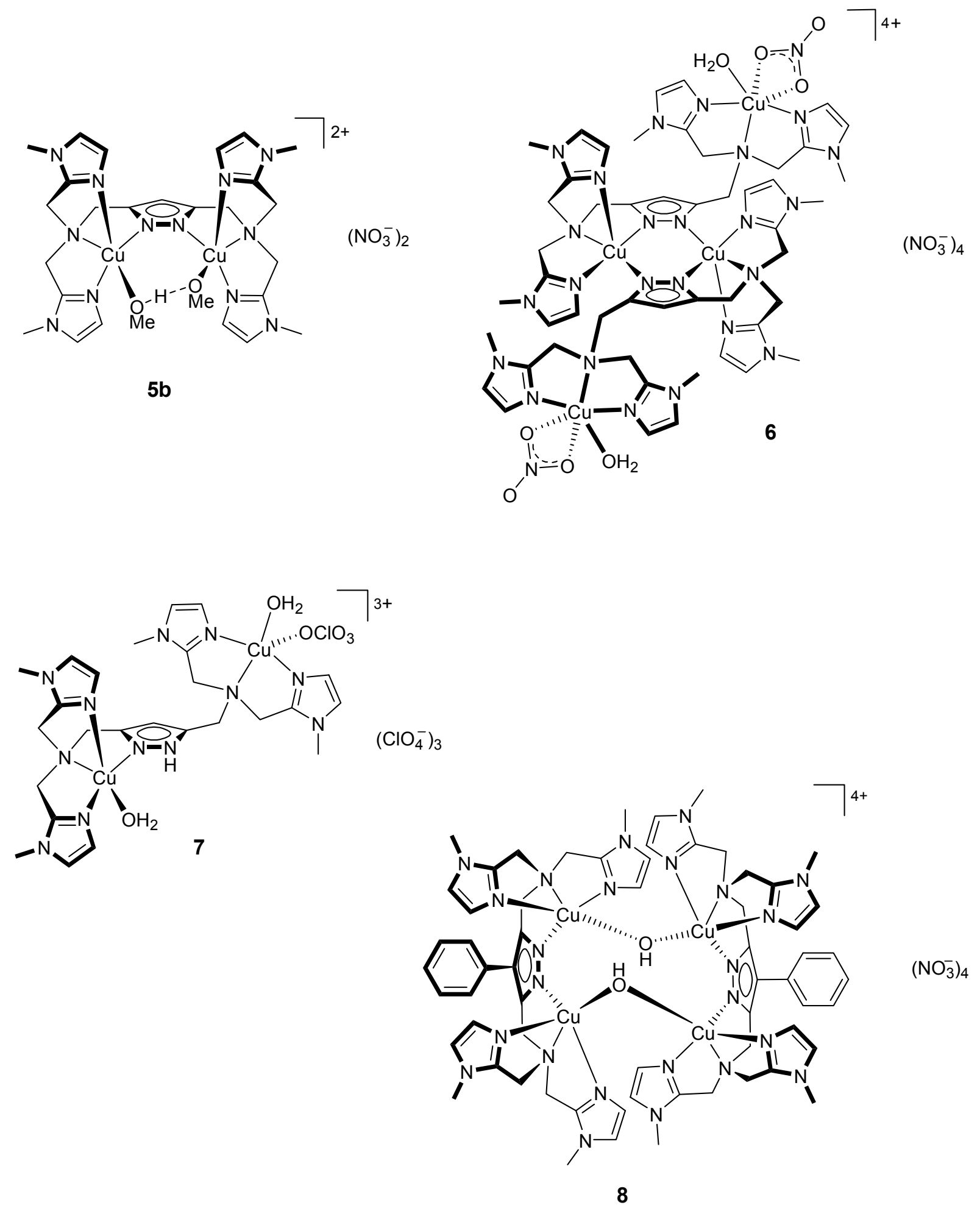

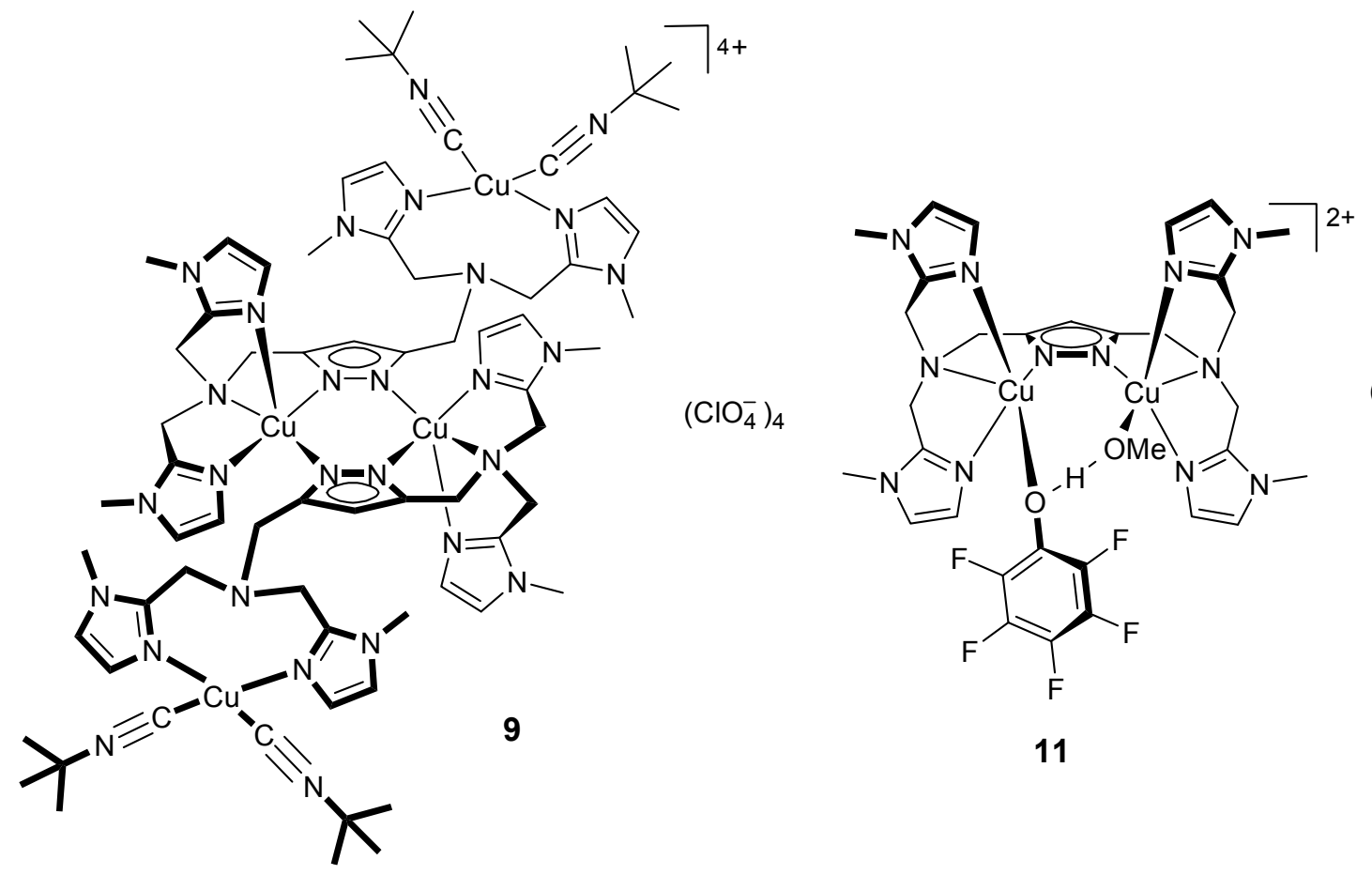

$\left(\mathrm{ClO}_{4}^{-}\right)_{2}$

11



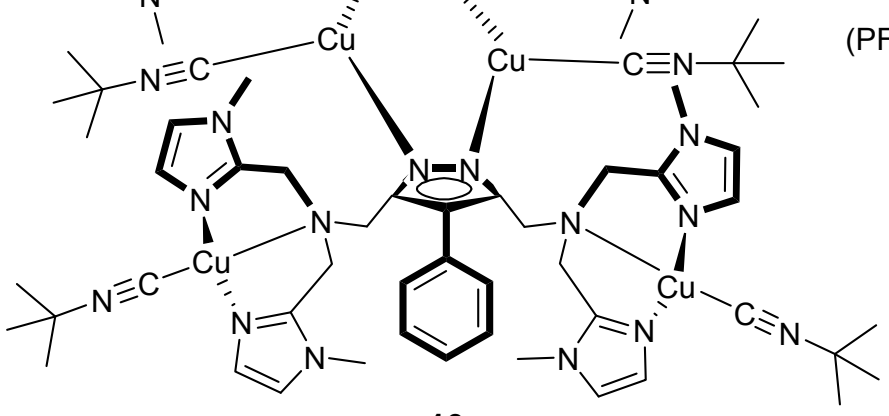<smiles>NC(=O)c1ccc(Cl)cc1</smiles>

12

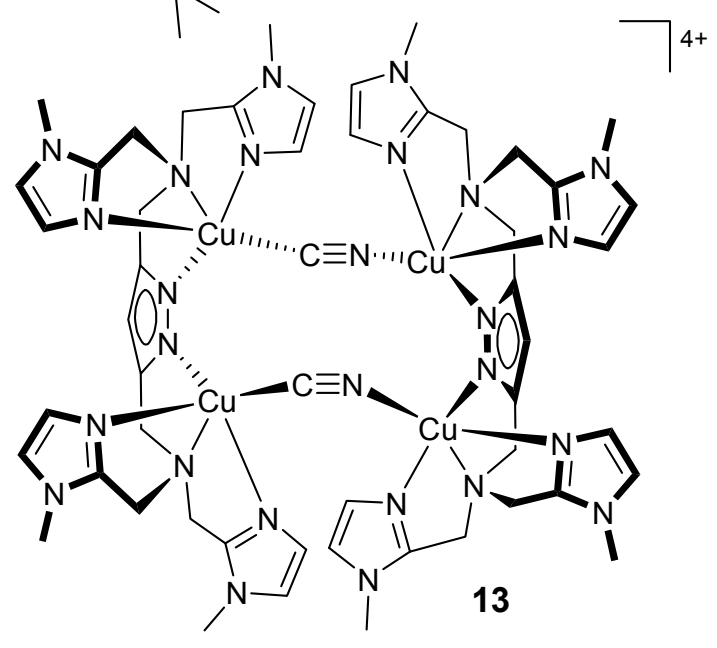

$\left(\mathrm{ClO}_{4}^{-}\right)_{4}$ 

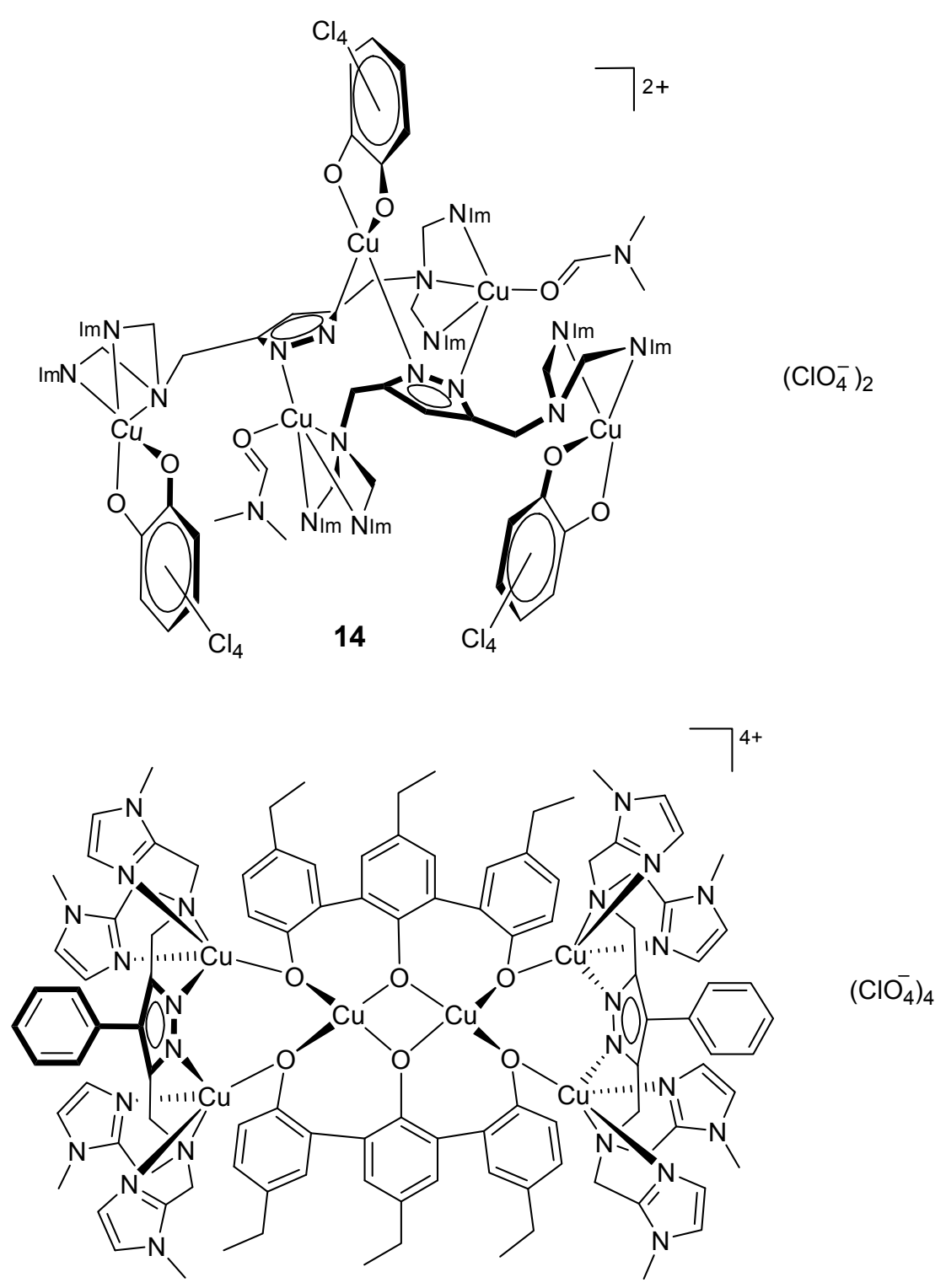

15

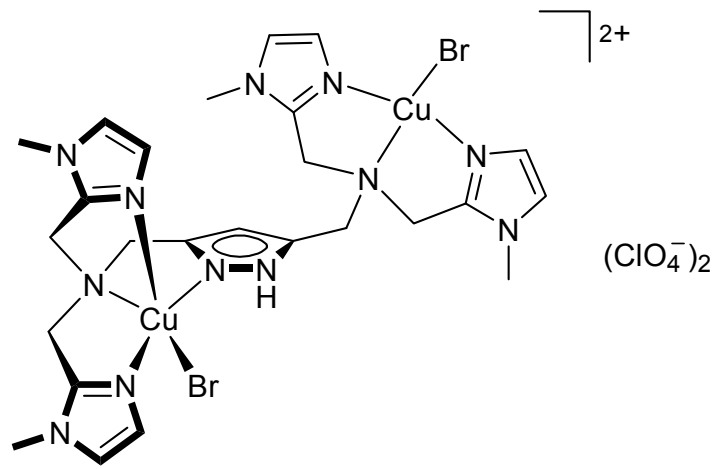




\section{Acknowledgments}

Very strange and exiting, this last part that has to be written, as it is anincredible part of the thesis where only heart and soul are participating and one can not find any formulas or figures, just feelings. You look at the word "Acknowledgments" and your skin is crawling because you are at the end....At the end of something very important and significant for the whole entire life.

"On this basis", I would like to direct my initial acknowledgment towards my supervisor Prof. Dr. Franc Meyer. I am truly grateful for a great and amazing chemistry-time here in Göttingen. I am thankful for an-in-time motivation moments which were so important and gave me the power to move on, even if it was hard and at some point impossible. All these moments gave me a chance to create a "golden bag" full with various chemical experiences and to open the world of science.

I would like to express my special thanks to Petra, who was incredibly kind and helpful in fixing all sorts of things during my whole time here. It was very important to be welcomed with warmth.

The whole working group will remain in my heart. My kind thanks to Rosanna and Jens, who were the first to show me my lab, my near future and the first synthesis of the dicopper(II) complex. And especially thank you for a nice time before the scary presentation in Ladenburg. My sincere thanks to Dr. Maringgele for fruitful discussions during the long evenings in the lab and for spending a lot of time showing me how to work with kilos of ligands. My lab-mates, Stamatia and Maria, are acknowledged for a nice time in lab 208 and for enjoying listening to Sirtaki. Many thanks to Jörg Teichgräber who was all the time around to help in the lab and to observe boiling solvents! I am thankful to all for a nice time (unfortunately, not that often) to hang around in Göttingen and during working trips. Dear Dr. Guido Leibeling is acknowledged for a great help in understanding of german laws and simply for a nice time! For the X-ray analyses I would like to acknowledge Dr. Sebastian Dechert who was very patient and showed endurance with all my "unexpected" structures; also thanks for the Raman measurements. My endless acknowledgments to the ukrainian part of the working group. Серёжа, большое спасибо за поддержку в течении этих четырёх лет. За весёлые чаяпития и шутки, за мудрые советы и дискуссии. За помощь по приезду в Гёттинген во всем. For the great help during a very important part of the thesis I want to thank Dr. Sasha Prykhod'ko. It was a big pleasure to work with you and I think that our team did well. For a nice half-a-year time in the lab, especially during early coming 
evenings, useful discussions and simply nice feelings bringing me closer to Kiev I am thankful to Larisa Penkova. To Dr. Michael Stollenz I want to say thanks for a nice time in practicum.

For the EPR measurements as well as for endless help with all administrative paper work I want to acknowledge Dr. Claudia Stückl. The divisions for NMR spectroscopy, mass spectrometry and elemental analysis I would like to acknowledge for their timely support during the research.

Financial support from the DFG (International Research Training Group GRK 1422 "Metal Sites in Biomolecules: Structures, Regulation and Mechanisms") and the Gottlieb Daimlerund Karl Benz-foundation for research fellowships is gratefully acknowledged.

For very productive and interesting seminars within the IRTG I want to thank to all people from the Inorganic Chemistry, Structural Biology, Plant Biology and Organic Chemistry departments, as well as for a nice and funny time during the Workshops in Kassel and Lund. In particular, I am thankful to Cristian Grosse from AK Sheldrick for X-Ray measurements. For a very heavy job, the corrections of the whole thesis I am grateful to Dr. Jarl Ivar van der Vlugt. I am thankful for veritably useful and helpful discussions concerning the scientific and the real world. For the nice company to hang out with in Göttingen and for a shared passion in playing pool! Dank je wel, Jarl!!!

I am thankful to my friends Lamiae, Mustapha, Mladen and Claudia for a lot of nice moments during these four years: for discussions on politics and culture, for shared human-being feelings coming from abroad, for funny times in the german course. I want to thank to my friend Olga for "cheer up" phone talks! For the light in the darkness, for care, for giving strength to believe in yourself, for a nice time during travelling around and just for being I am thankful to Anna.

Loving thanks to my family: to my dear cousin (sister) here and to all back home. For the endless support and love, for gigantic care on a thousand kilometres distance, which I felt every singe day, I want to thank my dear parents.

The trick is in what one emphasizes. We either make ourselves miserable, or we make ourselves strong. The amount of work is the same.

The Wheel of Time

Carlos Castaneda 


\section{Curriculum vitae}

Name

Date of birth:

Nationality:

Marital status:

Pre-Academic Education:

Academic Career:

Promotion:

Awards and Prizes:
Angelina Prokofieva

April $2^{\text {th }} 1982$, Kiev (Ukraine)

Ukrainian

Single

Sep 1988 - Jul 1998

High school № 294, Kiev, Ukraine

Sep 1998 - Jun 2002

Undergraduate Student at the Chemistry Department of the

National Taras Shevchenko University, Kiev, Ukraine Sep 2002 - Jun 2003

M.Sc. Student with honor at the Chemistry Department of the National Taras Shevchenko University, Kiev, Ukraine

Area: Crystal engineering: coordination polymers of $3 d-$ metals based on bipyrazolyl ligands

Nov 2003 - current

$\mathrm{Ph}$.D. Student at the Göttingen University, Institut of

Inorganic Chemistry Promotor: Prof.Dr. F.Meyer

Area: Modelling of the active site of type 3 copper

enzymes, bioinspired catalysis

Thesis title: "Bioinspired oxidation reactions of phenols with dinuclear copper complexes"

May 2003

First Prize at the Ukrainian students and post-graduate students conference

Sep 2004 - Aug 2006

Predoctoral Research Scholarship (Gottlieb Daimler- und Karl Benz-Stiftung)

25-30 June 2006

Prize for attendance of the $56^{\text {th }}$ Lindau Meeting of Nobel

Laureates ( $18^{\text {th }}$ Meeting dedicated to chemistry), Lindau,

Germany

Okt 2006 - Nov 2007

Scholarship within the International Research Training

Group (Internationales Graduirtenkolleg 1422) 


\section{Scientific publications}

A. Prokofieva, A. I. Prikhod'ko, E. A. Enyedy, E. Farkas, W. Maringgele, S. Demeshko, S. Dechert, F. Meyer, Oligonuclear copper complexes of a bioinspired pyrazolate-bridging ligand: synthesis, structures and equilibria in solution, Inorg.Chem., 2007, 46, 4298.

A. Prokofieva, A. I. Prikhod'ko, S. Dechert, F. Meyer, Selective benzylic C-C coupling catalyzed by a bioinspired dicopper complex, Hot Article, Chem.Commun., 2008, DOI: $10.1039 / \mathrm{b} 718162 \mathrm{k}$

A. Prokofieva, C. Große, G. M. Sheldrick, F. Meyer, Oxidative ortho C-C coupling of 4ethylphenol catalyzed by a bioinspired dicopper complex - trapping of an oligomeric intermediate, in preparation for Chem.Commun.

A. Prokofieva, A. I. Prikhod'ko, S. Schmatz, S. Dechert, M. Bennati, F. Meyer, Oxidative C-

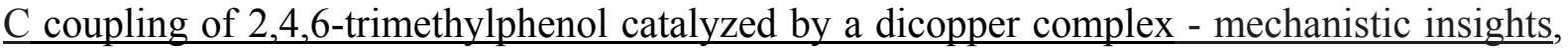
in preparation for Chem. Eur. J.

\section{Presentations at International Conferences and Workshops}

A. Prokofieva, K. Domasevich, "Coordination polymers of $3 d$-metals based on $N, N$ 'dimethyl-4,4'-bipyrazolyl", $4^{\text {th }}$ Ukrainian students and post-graduate students conference, Kiev, Ukraine, 21-23 May, 2003

A. Prokofieva, A. I. Prikhod'ko, F. Meyer, "Oxidative phenol coupling by pyrazolate-based dicopper complexes” 2. Koordinationschemikertreffen, Göttingen, Germany, 19-21 February, 2006

A. Prokofieva, A. I. Prikhod'ko, F. Meyer, "Bioinspired oxidation reactions with oligonuclear copper complexes ", $1^{\text {st }}$ workshop of the International Research Training Group, Reinhardswaldschule, Kassel, Germany, 25-27 January, 2007 


\section{Posters at International Conferences and Workshops}

A. Prokofieva, J. Ackermann, S. Dechert, F. Meyer, "Bioimimetic oxidation reactions with pyrazolate-based dicopper complexes", European Biological Inorganic Chemistry Conference (Eurobic7), Garmisch-Partenkirchen, Germany, 29 August-2 September, 2004

A. Prokofieva, J. Ackermann, S. Dechert, F. Meyer, "Bioinspired oxidation reactions with pyrazolate-based dicopper complexes," Workshop on Biomimetic Metal Oxidative Transformation, Marburg/Giessen, Germany, 29-30 January, 2005

A. Prokofieva, A. I. Prikhod'ko, S. Dechert, F. Meyer, "Bioinspired oxidation reactions with oligonuclear copper complexes," $2^{\text {nd }}$ workshop of the International Research Training Group, Lund, Sweden, 20-22 June, 2007

A. Prokofieva, A. I. Prikhod'ko, S. Dechert, F. Meyer, "Bioinspired oxidation reactions with oligonuclear copper complexes", $13^{\text {th }}$ International Conference on Biological Inorganic Chemistry (ICBIC), Vienna, Austria, 15-20 July, 2007 